\title{
PROBING MSUGRA WITH A SEARCH FOR CHARGINO-NEUTRALINO PRODUCTION USING TRILEPTONS
}

\author{
BY JULIAN GLATZER
}

\author{
A thesis submitted to the \\ Graduate School-New Brunswick \\ Rutgers, The State University of New Jersey \\ in partial fulfillment of the requirements \\ for the degree of \\ Master of Science \\ Graduate Program in Physics and Astronomy \\ Written under the direction of \\ Prof. Sunil Somalwar \\ and approved by
}

New Brunswick, New Jersey

October, 2008 


\title{
ABSTRACT OF THE THESIS
}

\section{Probing mSUGRA with a Search for Chargino-Neutralino Production using Trileptons}

\author{
by Julian Glatzer \\ Thesis Director: Prof. Sunil Somalwar
}

This thesis describes the CDF II $2 \mathrm{fb}^{-1}$ search for supersymmetric chargino-neutralino production in $\sqrt{s}=1.96 \mathrm{TeV} p \bar{p}$ collisions using the "golden" channel with three leptons and missing transverse energy in the final state and the application of the results to obtain a limit on the parameter space of the supersymmetric model mSUGRA.

Electrons and muons are reconstructed directly; isolated tracks are used as a proxy for $\tau$ leptons. Several analysis channels with different signal purity are defined and evaluated independently. Based on 7 observed and 6.4 expected background events this analysis was able set the first direct limits on chargino-neutralino production in mSUGRA since LEP. Based on mSUGRA phenomenology the sensitivity of this analysis is investigated; the mSUGRA parameter space is split into different phenomenology classes.

Experimental results from previous searches in this channel by CDF and D $\varnothing$ have been presented for specific parameter values of a given model. A more model-independent approach is proposed and, by splitting the results of this analysis into channels based on $\tau$ content, the results of the analysis are generalized. 


\section{Acknowledgements}

I want to express my deep gratitude to my advisor Prof. Sunil Somalwar. Thank you for supporting me at any time and keeping a clear overview whenever I feared to lose it. I have enjoyed the time as your student very much and have learnt from you far more than physics. I am much obliged to Sourabh Dube for being my supreme guide into high energy physics. This thesis would not have been possible in this way without your work and help. I also want to thank Alexander Sood for always being here, when help was needed, Prof. Scott Thomas for important help with all theory-related questions and numerous easily comprehensible explanations, Prof. Amitabh Lath, Prof. Eva Halkiadakis, Daryl Hare, Prof. Matthew Strassler and the whole high energy experiment group for a great time. At CDF I want to thank Prof. Benjamin Brau, Prof. Christopher Hays, Dr. Monica D'Onofrio and Prof. David Toback for their support and the pleasant environment in the CDF Exotic and SUSY working group. I want to thank the "Amerika-Programm" of the University of Würzburg and Rutgers University, especially Prof. Ronald Ransome, Shirley Hinds, Prof. Fakher Assaad and the Deutscher Akademischer Austauschdienst for making my stay at Rutgers University possible.

Friends are what make life worth living. Carolin, thanks for giving me all the compliments I don't deserve, but make me feel great. Jenny, thanks for even joining me in the most senseless talks and understanding all my kidding. Martin, thanks for being a great person to talk to about even the most absurd topics. Rebecca, thanks that I have always been able

to count on you. Silvia, thanks for being the only person in this world who appreciates my taste of music and for always being a great source of welcome distraction. I also want to thank Achim, Andrea, Johannes, Florian, Gencho, Mario, Miriam, Nina, Oliver, Sebastian, Sonja, Tim and all other people, which are missing in this list, for spending all these nice days and evenings with me and making my time in Würzburg so incredible. Thank you, Niki, for keeping an old and valued friendship alive even though we can't meet regularly. 
I also want to thank Robert and Jean-Patrick for sharing a lot of nice moments with me during my time at Rutgers.

Thank you, Corinne, for being with me for such a long time and knowing me like no one else does. Thank you for all your support, for the freedom I got from you and for bearing the side-effects when I am determined to achieve something. I want to thank my family, Benedikt, Christine and Manfred, for supporting me and being able to judge what is important in life - and what is not. I thank you for being the people you are, open-minded and supporting what is worth working and fighting for, but at the same time giving me freedom when I need it. Vielen Dank. 


\section{Dedication}

To life and all the liberal and open-minded people who make it so enjoyable. 


\section{Table of Contents}

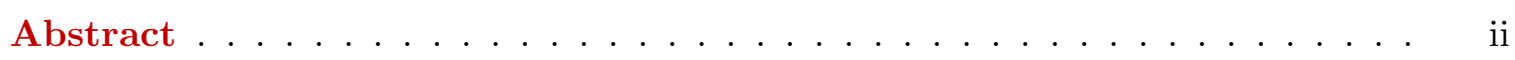

Acknowledgements .................... iii

Dedication ........................

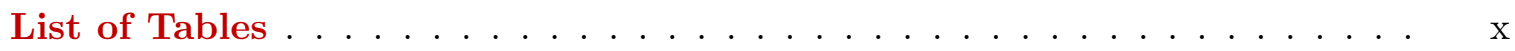

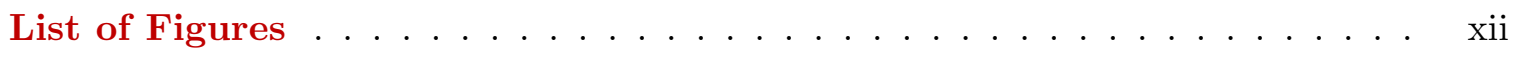

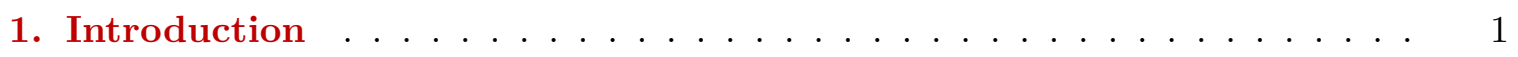

2. Overview and Theoretical Background . . . . . . . . . . 3

2.1. The Standard Model of Particle Physics . . . . . . . . . . . . . . . 3

2.1.1. Elementary Particles . . . . . . . . . . . . . . . . 3

2.1.2. Fundamental Interactions $\ldots \ldots \ldots$. . . . . . . . . 7

2.2. Supersymmetry ........................ 8

2.2.1. Particles of the Minimal Supersymmetric Standard Model . . . . . . 8

2.2.2. R-Parity . . . . . . . . . . . . . . . . 10

2.2.3. Supersymmetry Breaking . . . . . . . . . . . . . . 11

2.2.4. $\operatorname{mSUGRA} \ldots \ldots \ldots \ldots \ldots$

2.2.5. Neutralinos and Charginos . . . . . . . . . . . . 15

2.2.6. Supersymmetry and the Limitations of the Standard Model . . . . . 18

3. The Experimental Apparatus . . . . . . . . . . . . . . 23

3.1. The Tevatron and its Preaccelerators . . . . . . . . . . . 25

3.2. The Collider Detector at Fermilab . . . . . . . . . . . . 26

3.2.1. The Tracking System . . . . . . . . . . . . . . . 28 
3.2.2. The Calorimeter . . . . . . . . . . . . . . . 30

3.2.3. The Muon Chambers . . . . . . . . . . . . . . . . 32

3.2.4. The Trigger System . . . . . . . . . . . . . . . . . . 34

3.3. Datasets used in this Analysis . . . . . . . . . . . . . . 35

3.4. Monte Carlo Samples used in this Analysis . . . . . . . . . . . . . 36

4. The Trilepton Analysis . . . . . . . . . . . . . . . . 38

4.1. The Trilepton Signature . . . . . . . . . . . . . . . . . 38

4.2. Event Reconstruction . . . . . . . . . . . . . . . . . . 40

4.2.1. Event Vertex . . . . . . . . . . . . . . . . . 42

4.2.2. Tracks . . . . . . . . . . . . . . . . . . 42

4.2.3. Electrons . . . . . . . . . . . . . . . . . . 44

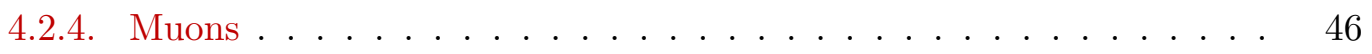

4.2.5. Jets . . . . . . . . . . . . . . . . . . . . 49

4.2.6. Identification Scale Factors . . . . . . . . . . . . . . 50

4.2.7. Missing Transverse Energy _. . . . . . . . . . . . . . . 50

4.2.8. Event Vetoes . . . . . . . . . . . . . . . . . . . 52

4.3. Definition of the Analysis Channels . . . . . . . . . . . . . 53

4.4. Standard Model and Non-physics Background . . . . . . . . . . . . 55

4.4.1. Background in the Trilepton Channels . . . . . . . . . . . . . . 55

4.4.2. Background in the Dilepton + Track Channels . . . . . . . . . 56

4.4.3. The Isolated Track Rate . . . . . . . . . . . . . . . . . 57

4.4.4. The Fake Rate . . . . . . . . . . . . . . . . 58

4.5. Control and Signal Regions . . . . . . . . . . . . . . . . 60

4.5.1. Definition . . . . . . . . . . . . . . . 60

4.5.2. Background Estimation . . . . . . . . . . . . . 61

4.6. Predictions and Results for the Signal Region . . . . . . . . . . . . . 64

4.6.1. Signal Optimization . . . . . . . . . . . . . . . . . . 64

4.6.2. Systematic Error . . . . . . . . . . . . . . . 66 
5. Interpretation of the Results in the mSUGRA Model . . . . . . . . . 71

5.1. Effects of the mSUGRA Parameters . . . . . . . . . . . . . 73

5.1.1. The Common Scalar Mass $m_{0} \ldots \ldots \ldots$. . . . . . . . . . 73

5.1.2. The Common Gaugino Mass $m_{1 / 2} \ldots \ldots \ldots$. . . . . . . 78

5.1.3. The Ratio of the Vacuum Expectation Values of the two Higgs Doublets $\tan \beta \ldots \ldots \ldots \ldots \ldots \ldots$. . . . . . . . . . . . . 82

5.1.4. The Common Trilinear Coupling $A_{0} \ldots \ldots \ldots$

5.2. Description of the Sensitivity of the Analysis . . . . . . . . . . . 88

5.2.1. Regions in mSUGRA Parameter Space . . . . . . . . . . . . 88

5.2.2. Cross Section for Associated Chargino-Neutralino Production . . . . 92

5.2.3. Branching Ratio of $\tilde{\chi}_{1}^{ \pm} \tilde{\chi}_{2}^{0}$ into Three Leptons . . . . . . . . . . . . . 92

5.2.4. Average Number of $\tau$ Leptons per Event . . . . . . . . . . . . . . 94

5.3. Limits on the Production Cross Section and the Chargino Mass . . . . . . 95

5.3.1. Calculation of a Limit on the Production Cross Section . . . . . . . 95

5.3.2. Limit on the Production Cross Section . . . . . . . . . . . 96

5.3.3. Limit on the Chargino Mass . . . . . . . . . . . . . . 98

5.4. Comparison of the Results to Previous Searches . . . . . . . . . . . . . . . 102

5.4.1. Results of LEP2 . . . . . . . . . . . . . . . . . . . . . . 102

5.4.2. CDF Results . . . . . . . . . . . . . . . . . . 103

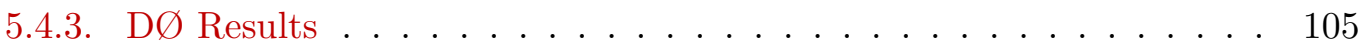

6. Model-Independent Interpretation of the Results . . . . . . . . . . . . 107

6.1. Description of the Method . . . . . . . . . . . . . . . 107

6.2. Determination of the Acceptance Functions . . . . . . . . . . . . . . 109

6.3. Verification of the Proposed Method in mSUGRA . . . . . . . . . . 113

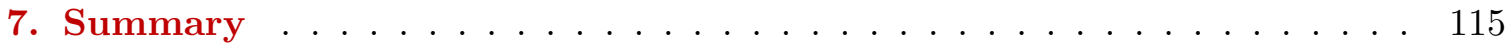

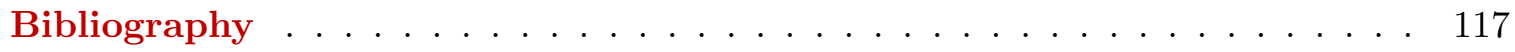




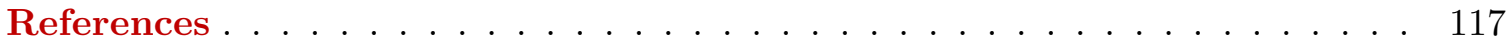

Appendix A. Control Regions . . . . . . . . . . . . . . . . . . 122

Appendix B. Limitations and Versions of Pythia and ISAJET . . . . . . . 124

Appendix C. Average Number of $\tau$ Leptons per Event . . . . . . . . . . 127

Appendix D. Monte Carlo Sample Generation for the Model-Independent

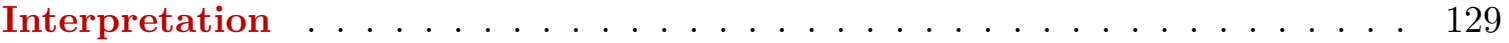

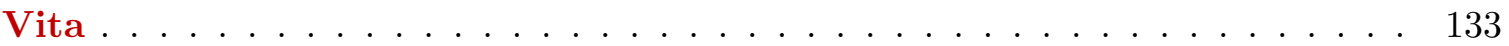




\section{List of Tables}

2.1. The elementary fermions . . . . . . . . . . . . . . . . 5

2.2. The four fundamental forces and their mediating gauge bosons $\ldots \ldots$. . . 7

3.1. Monte Carlo background samples . . . . . . . . . . . . . . . 36

4.1. Branching ratios for the different decay channels of the $\tau^{-}$lepton . . . . . . 41

4.2. Identification requirements for isolated tracks . . . . . . . . . . . . . . 42

4.3. Electron identification requirements . . . . . . . . . . . . 45

4.4. Muon identification requirements . . . . . . . . . . . . . . . 47

4.5. Lepton identification scale factors . . . . . . . . . . . . . . . 50

4.6. Definition of trilepton and dilepton analysis channels . . . . . . . . . . 54

4.7. Definition of control and signal regions . . . . . . . . . . . . . . 62

4.8. Background estimation . . . . . . . . . . . . . 66

4.9. Contributions to the systematic error . . . . . . . . . . . . . 68

4.10. Final number of expected signal and background events for the different analysis channels together with the observed number of events in data . . . 69

4.11. Characteristics of the observed events . . . . . . . . . . 70

5.1. Definition of the mSUGRA benchmark points and selected masses . . . . . 72

5.2. Mass of supersymmetric particles at benchmark point BP1 . . . . . . 76

5.3. Branching ratios for the different decay channels of the chargino at benchmark point $\mathrm{BP} 1 \ldots \ldots \ldots \ldots \ldots \ldots$. . . . . . . . . . . . . . . . 76

5.4. Mass of supersymmetric particles at benchmark point BP2 f . . . . . 76

5.5. Branching ratios for the different decay channels of the chargino at benchmark point $\mathrm{BP} 2 \ldots \ldots \ldots \ldots \ldots \ldots \ldots$

6.1. Selection criteria for the determination of the acceptance in the modelindependent approach . . . . . . . . . . . . . . . . . . . . 110 
6.2. Parameters of the acceptance fits of the model-independent interpretation .

6.3. Comparison of the actual acceptance from Pythia and the acceptance calculated using the fits . . . . . . . . . . . . . . . . . . 112

A.1. Number of expected and observed events for the trilepton control regions . 122

A.2. Number of expected and observed events for the dilepton control regions . . 123 


\section{List of Figures}

2.1. Evolution of sparticle masses as a function of the renormalization scale $Q$ for mSUGRA-like conditions . . . . . . . . . . . . . . . . . . . 14

2.2. Relevant allowed vertices for the decay of chargino and neutralino into leptons 18

2.3. Loop contributions to the scalar Higgs mass . . . . . . . . . . . . . . . . . 19

2.4. Evolution of the inverse gauge couplings as a function of the renormalization

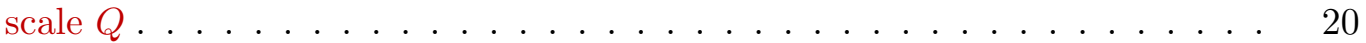

3.1. Aerial view of the Tevatron and the Main Injector . . . . . . . . . . . 23

3.2. Artistic illustration of the accelerator chain at Fermilab. . . . . . . . . . . . 24

3.3. Integrated luminosity delivered by the Tevatron accelerator . . . . . . . . . 24

3.4. The Collider Detector at Fermilab with opened plug . . . . . . . . . 26

3.5. Parts of the Collider Detector at Fermilab . . . . . . . . . . . . . . 27

3.6. The CDF tracking system . . . . . . . . . . . . . . 28

3.7. The CDF calorimeter system . . . . . . . . . . . . . . . . . 31

3.8. Coverage of the CDF muon detectors in the $(\eta, \phi) \ldots \ldots . \ldots 33$

4.1. Cross section for the production of different chargino and neutralino pairs . $\quad 38$

4.2. Leading order Feynman diagrams for the associated production of chargino and neutralino in $p \bar{p}$ collisions . . . . . . . . . . . . . . . . . . . . 39

4.3. Dominant decay channels of the neutralino $\tilde{\chi}_{2}^{0}$ and the chargino $\tilde{\chi}_{1}^{ \pm}$into leptons 41

4.4. Distribution of transverse momentum for dilepton + track events . . . . . . 43

4.5. Distribution of transverse momentum for trilepton events . . . . . . . . 48

4.6. Effects of successively applied corrections to missing $E_{T} \ldots \ldots \ldots$. . . . . 52

4.7. Measurement the isolated track rate . . . . . . . . . . . . . 57

4.8. Fake rates for TCE, LCE, CMUP, CMX and CMIO leptons . . . . . . . . 59

4.9. Illustration to the definition of signal and control regions . . . . . . . . 61 
4.10. Comparison of expected and observed number of events for the dilepton con-

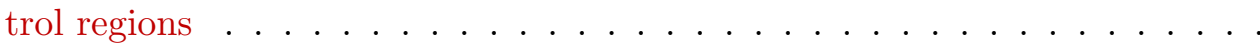

4.11. Comparison of expected and observed number of events for the trilepton control regions ......................... 63

4.12. Summary of expected and observed number of events in all control regions .

4.13. Invariant mass of the two leptons in the $l_{t} l_{t}$ analysis channel of the loMet dilepton control region . . . . . . . . . . . . . . . . .

4.14. Invariant mass of the two leptons in the $l_{t} l_{t} T$ analysis channel of the loMet trilepton control region . . . . . . . . . . . . . . . . . .

4.15. Missing transverse energy of events in the $l_{t} l_{t}$ analysis channel of the $\mathrm{Z}$ dilepton control region . . . . . . . . . . . . . . . .

4.16. Missing transverse energy of events in the $l_{t} l_{t} T$ analysis channel of the $\mathrm{Z}$ trilepton control region . . . . . . . . . . . . . . . . .

4.17. Signal and background N-1 plots for the signal optimization . . . . . . . .

5.1. Mass of the relevant supersymmetric particles as a function of $m_{0}$ at benchmark point BP3 with $\mu<0$ and at benchmark point BP1 with $\mu>0 \ldots$.

5.2. Branching ratios for the decay of chargino and neutralino as a function of $m_{0}$ at benchmark point $\mathrm{BP} 1$ with $\mu>0 \ldots \ldots \ldots$

5.3. Branching ratios for the decay of chargino and neutralino as a function of $m_{0}$ at benchmark point BP3 with $\mu<0 \ldots \ldots \ldots$

5.4. Branching ratio of $\tilde{\chi}_{1}^{ \pm} \tilde{\chi}_{2}^{0}$ into three leptons split into final states with $0,1,2$ or $3 \tau$ leptons as a function of $m_{0}$ at benchmark point BP3 with $\mu<0$ and at benchmark point BP1 with $\mu>0 \ldots \ldots \ldots$

5.5. Cross section for the production of $\tilde{\chi}_{1}^{ \pm} \tilde{\chi}_{2}^{0}$ in $p \bar{p}$ collisions at the Tevatron as a function of $m_{0}$ at benchmark point BP3 with $\mu<0$ and at benchmark point $\mathrm{BP} 1$ with $\mu>0 \ldots \ldots \ldots \ldots \ldots$

5.6. Mass of the relevant supersymmetric particles as a function of $m_{1 / 2}$ at benchmark point BP3 with $\mu<0$ and at benchmark point BP1 with $\mu>0 \ldots$ 
5.7. Branching ratios for the decay of chargino and neutralino as a function of $m_{1 / 2}$ at benchmark point BP1 with $\mu>0 \ldots \ldots$

5.8. Branching ratios for the decay of chargino and neutralino as a function of $m_{1 / 2}$ at benchmark point BP3 with $\mu<0 \ldots \ldots \ldots$

5.9. Branching ratio of $\tilde{\chi}_{1}^{ \pm} \tilde{\chi}_{2}^{0}$ into three leptons split into final states with $0,1,2$ or $3 \tau$ leptons as a function of $m_{1 / 2}$ at benchmark point BP3 with $\mu<0$ and at benchmark point BP1 with $\mu>0 \ldots \ldots \ldots$

5.10. Cross section for the production of $\tilde{\chi}_{1}^{ \pm} \tilde{\chi}_{2}^{0}$ in $p \bar{p}$ collisions at the Tevatron as a function of $m_{1 / 2}$ at benchmark point BP3 with $\mu<0$ and at benchmark point BP1 with $\mu>0 \ldots \ldots \ldots \ldots \ldots \ldots \ldots \ldots \ldots \ldots \ldots$

5.11. Mass of the relevant supersymmetric particles as a function of $\tan \beta$ at benchmark point BP3 with $\mu<0$ and at benchmark point BP1 with $\mu>0 \ldots$

5.12. Branching ratios for the decay of chargino and neutralino as a function of $\tan \beta$ at benchmark point BP1 with $\mu>0 \ldots \ldots \ldots$

5.13. Branching ratios for the decay of chargino and neutralino as a function of $\tan \beta$ at benchmark point BP3 with $\mu<0 \ldots \ldots$

5.14. Branching ratio of $\tilde{\chi}_{1}^{ \pm} \tilde{\chi}_{2}^{0}$ into three leptons split into final states with 0,1 , 2 or $3 \tau$ leptons as a function of $\tan \beta$ at benchmark point BP3 with $\mu<0$ and at benchmark point $\mathrm{BP} 1$ with $\mu>0 \ldots \ldots \ldots$

5.15. Cross section for the production of $\tilde{\chi}_{1}^{ \pm} \tilde{\chi}_{2}^{0}$ in $p \bar{p}$ collisions at the Tevatron as a function of $\tan \beta$ at benchmark point BP3 with $\mu<0$ and at benchmark point BP1 with $\mu>0 \ldots \ldots \ldots \ldots$

5.16. Mass of the relevant supersymmetric particles as a function of $A_{0}$ at benchmark point BP3 with $\mu<0$ and at benchmark point BP1 with $\mu>0 \ldots$

5.17. Branching ratios for the decay of chargino and neutralino as a function of $A_{0}$ at benchmark point $\mathrm{BP} 1$ with $\mu>0 \ldots \ldots \ldots$

5.18. Branching ratios for the decay of chargino and neutralino as a function of $A_{0}$ at benchmark point BP3 with $\mu<0 \ldots \ldots \ldots$ 
5.19. Branching ratio of $\tilde{\chi}_{1}^{ \pm} \tilde{\chi}_{2}^{0}$ into three leptons split into final states with $0,1,2$ or $3 \tau$ leptons as a function of $A_{0}$ at benchmark point BP3 with $\mu<0$ and at benchmark point $\mathrm{BP} 1$ with $\mu>0 \ldots \ldots \ldots$

5.20. Cross section for the production of $\tilde{\chi}_{1}^{ \pm} \tilde{\chi}_{2}^{0}$ in $p \bar{p}$ collisions at the Tevatron as a function of $A_{0}$ at benchmark point BP3 with $\mu<0$ and at benchmark point BP1 with $\mu>0 \ldots \ldots \ldots \ldots \ldots \ldots \ldots$

5.21. mSUGRA regions with different phenomenology classes $\ldots \ldots \ldots$. . . 88

5.22. Cross section for the production of an associated chargino-neutralino pair in $\sqrt{s}=1.96 \mathrm{TeV} p \bar{p}$ collisions at the Tevatron as a function of $m_{0}$ and $m_{1 / 2}$.

5.23. Branching ratio for the decay of a chargino-neutralino pair into three leptons

5.24. Mean number of $\tau$ leptons as a function of $m_{0}$ and $m_{1 / 2} \ldots \ldots$. . .

5.25. Monte Carlo points used for obtaining a limit on the $\tilde{\chi}_{1}^{ \pm} \tilde{\chi}_{2}^{0}$ production cross

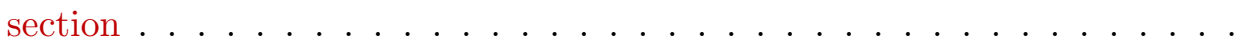

5.26. Observed limit on the production cross section multiplied with the branching ratio of $\tilde{\chi}_{1}^{ \pm} \tilde{\chi}_{2}^{0}$ into three leptons . . . . . . . . . . .

5.27. Limit on the mass of the chargino at $m_{0}=60 \mathrm{GeV} / \mathrm{c}^{2}$ in region $\mathrm{B}$, where two-body decays are dominant. . . . . . . . . . . . . . . .

5.28. Limit on the mass of the chargino at $m_{0}=100 \mathrm{GeV} / \mathrm{c}^{2}$ in region $\mathrm{A}$, where three-body decays are dominant. . . . . . . . . . . . . . . .

5.29. Interpolation of the observed upper limit and the theory cross section and branching ratio into three leptons to obtain an exclusion region in mSUGRA 100

5.30. $95 \%$ confidence level exclusion region in mSUGRA . . . . . . . . . . . 101

5.31. Overlay of the theoretical production cross section multiplied by the branching ratio of $\tilde{\chi}_{1}^{ \pm} \tilde{\chi}_{2}^{0}$ into three leptons and the exclusion region in mSUGRA .

5.32. Excluded regions in mSUGRA by the LEP experiments . . . . . . . . . . . 104

5.33. Excluded chargino masses for CDF and D . . . . . . . . . . . 105

6.1. Analysis acceptance split into channels according to the number of $\tau$ leptons in the trilepton final state $\ldots \ldots \ldots \ldots$ 
6.2. Exclusion region in mSUGRA obtained with the proposed model-independent

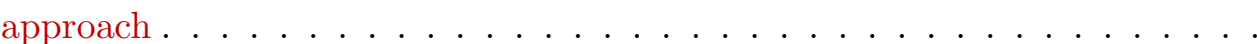

B.1. Branching ratio of chargino and neutralino into three leptons at the transition from off-shell three-body decay to on-shell sequential two-body decay . . . .

B.2. Branching ratio of chargino and neutralino into three leptons . . . . . . .

B.3. Branching ratios for the chargino as a function of $m_{1 / 2}$ at benchmark point

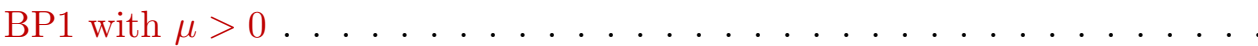

C.1. Average number of $\tau$ leptons per event as a function of $m_{0}$ at benchmark point BP1 with $\mu>0 \ldots \ldots \ldots \ldots \ldots$

C.2. Average number of $\tau$ leptons per event as a function of $m_{0}$ at benchmark point BP3 with $\mu<0 \ldots \ldots \ldots \ldots \ldots$

C.3. Average number of $\tau$ leptons per event as a function of $m_{1 / 2}$ at benchmark point BP1 with $\mu>0 \ldots \ldots \ldots \ldots$

C.4. Average number of $\tau$ leptons per event as a function of $m_{1 / 2}$ at benchmark point BP3 with $\mu<0 \ldots \ldots \ldots \ldots \ldots$

C.5. Average number of $\tau$ leptons per event as a function of $\tan \beta$ at benchmark point $\mathrm{BP} 1$ with $\mu>0 \ldots \ldots \ldots \ldots$

C.6. Average number of $\tau$ leptons per event as a function of $\tan \beta$ at benchmark point BP3 with $\mu<0 \ldots \ldots \ldots \ldots \ldots$

C.7. Average number of $\tau$ leptons per event as a function of $A_{0}$ at benchmark point BP1 with $\mu>0 \ldots \ldots \ldots \ldots \ldots$

C.8. Average number of $\tau$ leptons per event as a function of $A_{0}$ at benchmark point BP3 with $\mu<0 \ldots \ldots \ldots \ldots \ldots$ 


\section{Chapter 1 \\ Introduction}

"There's Plenty of Room at the Bottom"1

Richard P. Feynman

The universe, stars, planets, oceans, mountains, trees and humans, everything around us is made of matter. The question, what the universe and all matter is made of and if there is a smallest constituent, that makes up all matter, is one of the oldest questions that has been asked by humanity. The idea that the universe is made of small and simple building blocks is old and has probably first come up in the 6th century BC in ancient India, even though the idea is now often ascribed to Democritus. To search at a very small distance scale very high energies are necessary. In high energy physics experiments particles such as electrons or protons are accelerated to nearly the speed of light. In a collision of two particles, very high energy densities, similar to the ones that existed right after the Big Bang, are created and smaller structures, that might make up our matter, can be revealed.

What is mass? What is dark matter? Are there any other fundamental particles we have not yet seen? Are all forces low-energy realizations of a single force at a higher energy? Particle physics might be able to find first answers to these questions in the next years. The Tevatron at Fermilab near Chicago and the Large Hadron Collider at CERN near Geneva are two particle physics experiments that can accelerate protons to energies where secondary particle can be produced that no other experiment was ever able to see. The Higgs boson might give an answer to how particles acquire mass and supersymmetry - if realized in nature - doubles the number of elementary particles and might explain the nature of dark

\footnotetext{
1 "There's Plenty of Room at the Bottom" is the title of a talk given by Richard P. Feynman. Even though Feynman's talk was on the topic of nanophysics the idea is also applicable at the even smaller scale of particle physics.
} 
matter. Supersymmetry might be the next step towards a deeper understanding of the fundamental symmetries and laws of nature.

This thesis will document a search for supersymmetry at the Collider Detector at Fermilab (CDF II). At the Tevatron protons collide with antiprotons at a center-of-mass energy of $1.96 \mathrm{TeV}$ and a pair of supersymmetric particles, the chargino and the neutralino, may be produced. They may subsequently decay into three leptons along with weakly interacting particles, that can be seen in the detector as missing transverse energy. The Standard Model of Particle Physics is currently the best and very successful description of most experimental observations in high energy physics. As the Standard Model predicts a low number of events with three leptons and missing transverse energy in the final state, this signature is a good experimental probe to discover deviations from the Standard Model. Even though the signature with which this search is carried out is tailored towards a special realization of supersymmetry, it is possible to see deviations from the Standard Model due to other models.

\section{A Note on Units and used Terminology}

In particle physics it is commonly accepted to use units where $\hbar=c=1$. In this thesis units are determined up to powers of $\hbar$ and $c$; however in special cases powers of $\hbar$ and $c$ are given.

The term neutralino will be used for the next-to-lightest neutralino $\tilde{\chi}_{2}^{0}$; by chargino we denote the lighter chargino $\tilde{\chi}_{1}^{ \pm}$and by $\operatorname{LSP}^{2}$ the lightest neutralino $\tilde{\chi}_{1}^{0}$. The term lepton will be used context-dependent and includes electrons and muons or electrons, muons and $\tau$ leptons or electrons, muons, $\tau$ leptons and the matching neutrinos.

\footnotetext{
${ }^{2}$ LSP is an abbreviation for lightest supersymmetric particle. In the parameter space considered in this analysis the lightest neutralino is the lightest supersymmetric particle.
} 


\section{Chapter 2 \\ Overview and Theoretical Background}

\subsection{The Standard Model of Particle Physics}

The Standard Model of Particle Physics (SM) describes the electromagnetic, the weak and the strong interaction between the elementary particles of which all matter consists. It is formulated as a relativistic quantum field theory using the gauge group

$$
\mathrm{U}(1)_{\mathrm{Y}} \times \mathrm{SU}(2)_{\mathrm{L}} \times \mathrm{SU}(3)_{\mathrm{C}}
$$

as an abstract description of the interactions. The basis of the Standard Model of Particle Physics was developed in the first half of the 1970's and confirmed in numerous experimental probes.

\subsubsection{Elementary Particles}

The elementary particles in the Standard Model of Particle Physics can be divided into bosons and fermions. Bosons have integer spin $(J \in\{0,1,2,3, \ldots\})$ and act as the carriers of the four fundamental forces of nature, whereas fermions are particles with half-integer

spin $\left(J \in\left\{\frac{1}{2}, \frac{3}{2}, \frac{5}{2}, \ldots\right\}\right)$. The interactions of fermions are described by gauge theories and are mediated by the exchange of gauge bosons.

\section{Elementary Fermions}

The elementary particles our normal matter is made of are fermions. Examples for these particles are electrons and the up and down quark of which the proton and neutron are made. Elementary fermions have $\operatorname{spin} J=\frac{1}{2}$.

Elementary fermions can be categorized into quarks, which can participate in the strong, the weak and the electromagnetic interaction, and leptons, which can participate in the weak 
and the electromagnetic interaction only. The elementary fermions can be ordered in three generations, where in the first generation up and down quark as well as electron and electron neutrino are, in the second generation charm and strange quark as well as muon and muon neutrino and in the third generation bottom and top quark as well as tau and tau neutrino. A schematic ordering of these particles can be found in Table 2.1.

Quarks (antiquarks) carry a baryon quantum number of $B=1 / 3(B=-1 / 3)$, which is approximately conserved even though a violation has not yet been observed. A quarkantiquark pair can form a meson $(B=0)$ and three quarks can form a baryon $(B= \pm 1)$. Other combinations of quarks have not yet been observed. Protons, made of two up and one down quark, and neutrons, made of one up and two down quarks, are baryons. As quarks are spin $1 / 2$ particles they can occur in a left- and right-handed form according to the orientation of their spin. The right-handed quarks form weak-isospin singlets, the left-handed quarks of one generation form a weak-isospin doublet.

Leptons (antileptons) carry a lepton quantum number of $L=1(L=-1)$, which is approximately conserved even though a violation has not yet been observed ${ }^{1}$. The difference of baryon and lepton quantum number $B-L$ is absolutely conserved. The left-handed leptons and the neutrino of the same flavor form a weak-isospin doublet. The right-handed lepton forms a weak-isospin singlet. In the Standard Model there is no right-handed neutrino, however newer experiments suggest non-vanishing neutrino masses, which might be a reason for a right-handed neutrino to exist.

\section{Elementary Gauge Bosons}

The gauge bosons that are included in the Standard Model of Particle Physics are the photon $\gamma$, which is mediating the electromagnetic interaction, the $W^{+}, W^{-}, Z^{0}$ bosons, which are mediating the weak force, and the gluon $g$, which is mediating the strong color force. The hypothetical graviton $G$, which might be mediating gravity is not included in the Standard Model. An overview of the properties of the elementary gauge bosons can be found in Table 2.2.

\footnotetext{
${ }^{1} \mathrm{~A}$ violation of the lepton family numbers has been observed in the process of neutrino oscillations.
} 
Table 2.1: The elementary fermions and their masses ${ }^{a}$

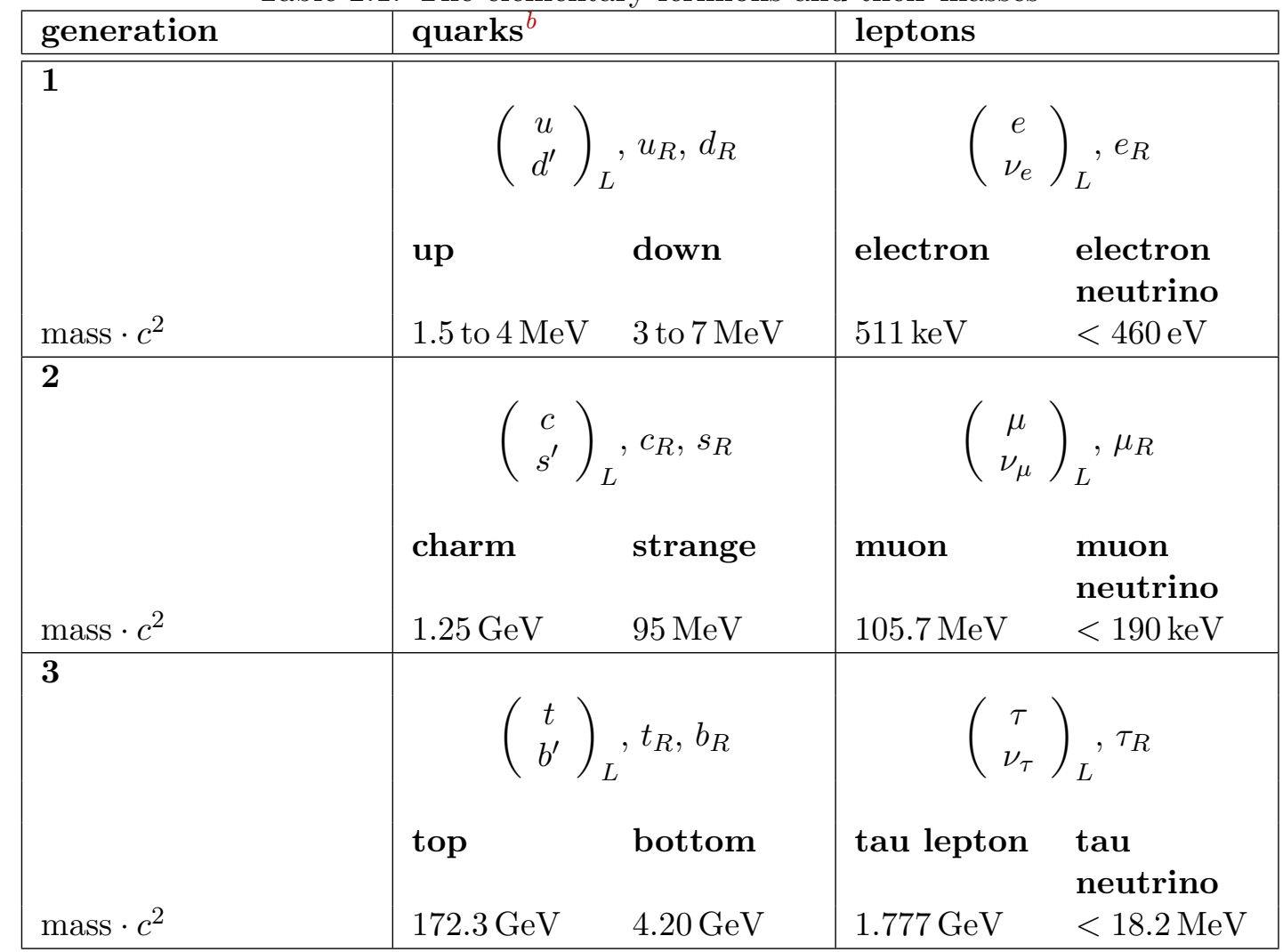

${ }^{a}$ Antiparticles are not listed. Masses are according to [1].

${ }^{b}$ The down-type quarks appearing in the doublets $d^{\prime}, s^{\prime}$ and $b^{\prime}$ are interaction eigenstates. Mass eigenstates are obtained by diagonalizing the Cabibbo-Kobayashi-Maskawa matrix[2]. 
The Standard Model Lagrangian is built of kinetic terms of the form

$$
\mathcal{L}=\imath \Psi^{\dagger} \not D \Psi
$$

where $\Psi$ is a Dirac spinor, for all fermions and terms for the potential energy and interactions. The gauge bosons enter the Lagrangian in the definition of

$$
\not D=\gamma^{\mu} D_{\mu}, \quad D_{\mu}=\partial_{\mu}-\frac{\imath}{2} g_{2} W_{\mu}^{a} \sigma^{a}-\imath g_{Y} Y_{\phi} B_{\mu},
$$

where the fields $W_{\mu}$ and $B_{\mu}$ mix to form the $W^{+}, W^{-}, Z^{0}$ bosons and the photon, $\gamma^{\mu}$ are the Dirac and $\sigma^{a}$ the Pauli matrices; $g_{2}$ and $g_{Y}$ are the coupling parameters for the $U(1)$ electromagnetic and the $S U(2)$ weak interaction. A more complete description of the Standard Model Lagrangian and the syntax used here can be found in [3].

\section{The Higgs Boson}

The Higgs boson is a hypothetical particle that can explain the origin of mass. In its minimal Standard Model version it is represented by a scalar field $\phi=\left(\phi_{1}, \phi_{2}\right)^{T}$ and yields the Lagrangian contribution[4]

$$
\mathcal{L}_{\phi}=\left(D_{\mu} \phi\right)^{\dagger} D_{\mu} \phi-\mu^{2} \phi^{\dagger} \phi-\lambda\left(\phi^{\dagger} \phi\right)^{2}
$$

The gauge symmetry of the Lagrangian is broken when a specific vacuum expectation value is chosen to minimize the potential:

$$
\langle\phi\rangle=\frac{1}{\sqrt{2}}\left(\begin{array}{c}
0 \\
v
\end{array}\right) \quad, \text { where } v=\sqrt{\frac{-\mu^{2}}{\lambda}} \quad \mu^{2}<0, \lambda>0 .
$$

The term $\left(D_{\mu} \phi_{i}\right)^{2}$ now includes the term

$$
\underbrace{\frac{1}{4} g_{2}^{2}\left(\sigma^{a} \phi\right)_{i}\left(\sigma^{b} \phi\right)_{i}}_{=m_{a b}^{2} \text { for } \phi=\phi_{0}} A_{\mu}^{a} A^{\mu b}
$$

where $m_{a b}^{2}$, after diagonalization, results in the boson masses

$$
m_{W}^{2}=\frac{1}{4} g_{2}^{2} v^{2}, \quad m_{Z}^{2}=\frac{1}{4}\left(g_{2}^{2}+g_{Y}^{2}\right) v^{2}
$$

Fermion masses result from a Yukawa coupling to the Higgs boson doublet. 
Table 2.2: The four fundamental forces and their mediating gauge bosons ${ }^{a}[5]$.

\begin{tabular}{|l|l|l|l|l|}
\hline Force & Gravity $^{b}$ & $\begin{array}{l}\text { Electromagnetic } \\
\text { Force }\end{array}$ & Weak Force & Strong Force \\
\hline \hline $\begin{array}{l}\text { Field Boson } \\
\text { Spin- }\end{array}$ & Graviton G & Photon $\gamma$ & $\mathrm{W}^{ \pm}, Z^{0}$ & Gluon $\mathrm{g}$ \\
Parity & $2^{+}$ & $1^{-}$ & $1^{-}, 1^{+}$ & $1^{+}$ \\
Mass & 0 & 0 & $80.4 \mathrm{GeV}(W)$, & 0 \\
range & $\infty$ & $\infty$ & $91.2 \mathrm{GeV}(Z)$ & $\sim 10^{-18} \mathrm{~m}$ \\
source & mass & electric charge & 'weak charge' & 'color charge' \\
coupling & $\frac{G_{N} M^{2}}{4 \pi \hbar c}$ & $\alpha=\frac{e^{2}}{4 \pi \hbar c}$ & $\frac{G\left(M c^{2}\right)^{2}}{(\hbar c)^{3}}$ & $\alpha_{s} \leq 1$ \\
constant & $\approx 5 \cdot 10^{-40}$ & $\approx \frac{1}{137}$ & $\approx 1.17 \cdot 10^{-5}$ & \\
(effective) & $\propto \frac{1}{r}$ & $\propto \frac{1}{r}$ & $\propto \frac{\exp -m_{W, Z^{r}}}{r}$ & $\propto K r-\frac{\alpha}{r}$ \\
potential & $\propto \frac{1}{r}$ & &
\end{tabular}

${ }^{a}$ Numbers are calculated for for $M c^{2}=1 \mathrm{GeV}$.

${ }^{b}$ Gravity is not included in the Standard Model of Particle Physics; the graviton is a hypothetical particle.

\subsubsection{Fundamental Interactions}

As of now the four known fundamental forces are: the strong force, the weak force, the electromagnetic force and gravity. Except for gravity, all fundamental interactions can be described within the framework of the Standard Model of Particle Physics. The electromagnetic force and the weak force can be described by the electroweak theory which is a unified theory of quantum electrodynamics and the weak interaction. The strong force can be described by quantum chromodynamics. As long as energies are well below the Planck scale $M_{p}$ effects of gravity can be neglected ${ }^{2}$, but the fact that the Standard Model of Particle Physics has to break down at energies comparable to the Planck scale can be taken as a hint that the Standard Model is an effective theory for energies smaller than the Planck scale.

\footnotetext{
${ }^{2}$ It is $M_{P}=\sqrt{\frac{\hbar c}{G_{N}}} \approx 1.22 \cdot 10^{28} \mathrm{eV}$.
} 


\subsection{Supersymmetry}

Supersymmetry is a proposed symmetry, which relates bosons and fermions. The supersymmetry operator $Q_{r}$ acts on bosons (fermions) according to

$$
\left.\left.\left.\left.Q_{r} \mid \text { boson }\right\rangle=\mid \text { fermion }\right\rangle \quad Q_{r} \mid \text { fermion }\right\rangle=\mid \text { boson }\right\rangle
$$

and introduces a superpartner for every Standard Model particle. The superpartner differs from the Standard Model particle by spin $\frac{1}{2}$, so that the superpartner for a boson is a fermion and the superpartner for a fermion is a boson.

\subsubsection{Particles of the Minimal Supersymmetric Standard Model}

The Minimal Supersymmetric Standard Model (MSSM) tries to parameterize a model with minimal particle content and soft supersymmetry breaking in a way as general as possible. In the flavor sector alone it has 110 free parameters: 30 masses, 39 real mixing angles and 41 phases[6].

While in the Standard Model one scalar Higgs boson is enough to generate masses, in the Minimal Supersymmetric Standard Model two Higgs doublets

$$
H_{u}=\left(\begin{array}{c}
H_{u}^{+} \\
H_{u}^{0}
\end{array}\right), \quad H_{d}=\left(\begin{array}{c}
H_{d}^{0} \\
H_{d}^{-}
\end{array}\right)
$$

are needed. The vacuum expectation values of the two Higgs doublets are related to the Standard Model Z boson mass and the couplings $g_{2}$ and $g_{Y}$ via Eqn. 2.7, so that

$$
\left\langle H_{u}\right\rangle^{2}+\left\langle H_{d}\right\rangle^{2}=v_{u}^{2}+v_{d}^{2}=v^{2}=\frac{2 m_{Z}^{2}}{g_{Y}^{2}+g_{2}^{2}}=\frac{2 m_{W}^{2}}{g_{2}^{2}} \approx(174 \mathrm{GeV})^{2}[7] .
$$

The ratio of the vacuum expectation values of the two Higgs doublets

$$
\tan \beta=\frac{v_{u}}{v_{d}}
$$

is a free parameter of the theory. As all components of the two doublets can be complex, eight degrees of freedom exist[8] and mix to form the five physical states of the Higgs 
spectrum $H^{ \pm}, A^{0}, h^{0}, H^{0}$ and the three Goldstone bosons, that provide the longitudinal components of the massive $W^{ \pm}$and $Z^{0}$ bosons via the Higgs mechanism. It is

$$
\begin{aligned}
H^{ \pm} & =H_{d}^{ \pm} \sin \beta+H_{u}^{ \pm} \cos \beta, \\
A^{0} & =\sqrt{2}\left(\operatorname{Im} H_{d}^{0} \sin \beta+\operatorname{Im} H_{u}^{0} \cos \beta\right), \\
h^{0} & =-\left(\sqrt{2} \operatorname{Re} H_{d}^{0}-v_{d}\right) \sin \alpha+\left(\sqrt{2} \operatorname{Re} H_{u}^{0}-v_{u}\right) \cos \alpha, \\
H^{0} & =\left(\sqrt{2} \operatorname{Re} H_{d}^{0}-v_{d}\right) \cos \alpha+\left(\sqrt{2} \operatorname{Re} H_{u}^{0}-v_{u}\right) \sin \alpha .
\end{aligned}
$$

The superpartners of the Higgs bosons are the Higgsinos. In a superfield formulation usual mass terms for the Higgs superfields $H_{u}, H_{d}$ can not be introduced as the superpotential has to be analytic in the superfields. In the supersymmetric Lagrangian the term

$$
\mu H_{u} H_{d}
$$

where $\mu$ is the Higgs mixing parameter, is introduced to ensure electroweak symmetry breaking[9] and give mass to all quarks and leptons. Together with the Bino $\tilde{B}^{0}$ and the neutral Wino $\tilde{W}^{0}$ the neutral Higgsinos $\tilde{H}_{d}^{0}$ and $\tilde{H}_{u}^{0}$ form mass eigenstates, the so-called neutralinos $\tilde{\chi}_{i}^{0}, i=1,2,3,4$. The charged Higgsinos $\tilde{H}^{ \pm}$together with the charged Winos $\tilde{W}^{ \pm}$also mix and form mass eigenstates, the charginos $\tilde{\chi}_{i}^{ \pm}, i=1,2$.

In the Standard Model six doublets, containing the three generations of left-handed leptons and quarks $\left(e_{i}, \nu_{i}\right)_{L},\left(u_{i}, d_{i}\right)_{L}$, and nine singlets, containing the right-handed leptons and quarks $e_{i, R}, u_{i, R}, d_{i, R}$, exist. According to section 2.2 for every Standard Model doublet a supersymmetric doublet and for every Standard Model singlet a supersymmetric singlet exists, containing the selectrons, smuons, stau sleptons and squarks

$$
\begin{array}{r}
\left(\tilde{e}, \tilde{\nu}_{e}\right)_{L},\left(\tilde{\mu}, \tilde{\nu}_{\mu}\right)_{L},\left(\tilde{\tau}, \tilde{\nu}_{\tau}\right)_{L}, \tilde{e}_{R}, \tilde{\mu}_{R}, \tilde{\tau}_{R} \\
(\tilde{u}, \tilde{d})_{L},(\tilde{c}, \tilde{s})_{L},(\tilde{t}, \tilde{b})_{L}, \tilde{u}_{R}, \tilde{d}_{R}, \tilde{c}_{R}, \tilde{s}_{R}, \tilde{t}_{R}, \tilde{b}_{R}
\end{array}
$$

The superpotential of the MSSM is[7]

$$
W_{\mathrm{MSSM}}=\bar{u}_{R} \mathbf{y}_{\mathbf{u}} Q_{L} H_{u}-\bar{d}_{R} \mathbf{y}_{\mathbf{d}} Q_{L} H_{d}-\bar{e}_{R} \mathbf{y}_{\mathbf{e}} L_{L} H_{d}+\mu H_{u} H_{d},
$$


where $H_{u}, H_{d}, Q_{L}, L_{L}, \bar{u}_{R}, \bar{d}_{R}, \bar{e}_{R}$ are chiral superfields and $\mathrm{L}, \mathrm{R}$ denotes left- and righthanded particle content. $\mathbf{y}_{\mathbf{u}}, \mathbf{y}_{\mathbf{d}}, \mathbf{y}_{\mathbf{e}}$ are the Yukawa matrices and in the approximation that

$$
\mathbf{y}_{\mathbf{u}} \approx\left(\begin{array}{ccc}
0 & 0 & 0 \\
0 & 0 & 0 \\
0 & 0 & y_{t}
\end{array}\right), \mathbf{y}_{\mathbf{d}} \approx\left(\begin{array}{ccc}
0 & 0 & 0 \\
0 & 0 & 0 \\
0 & 0 & y_{b}
\end{array}\right), \mathbf{y}_{\tau} \approx\left(\begin{array}{ccc}
0 & 0 & 0 \\
0 & 0 & 0 \\
0 & 0 & y_{\tau}
\end{array}\right)
$$

the superpotential is

$$
W_{\mathrm{MSSM}} \approx y_{t}\left(\bar{t} t H_{u}^{0}-\bar{t} b H_{u}^{+}\right)-y_{b}\left(\bar{b} t H_{d}^{-}-\bar{b} b H_{d}^{0}\right)-y_{\tau}\left(\bar{\tau} \nu_{\tau} H_{d}^{-}-\bar{\tau} \tau H_{d}^{0}\right)+\mu\left(H_{u}^{+} H_{d}^{-}-H_{u}^{0} H_{d}^{0}\right)
$$

In this approximation it can be seen that the third generation superfields have a coupling to the Higgs superfields while the first and second generation superfields do not. The consequence of this approximation is that the first and second generation gauge and mass eigenstates are approximately equal while left- and right-handed sfermions of the third generation mix to form the sfermions

$$
\tilde{\tau}_{1}, \tilde{\tau}_{2}, \tilde{\nu}_{\tau}, \tilde{t}_{1}, \tilde{t}_{2}, \tilde{b}_{1}, \tilde{b}_{2}
$$

According to [7], the degree of mixing depends on the ratio of the vacuum expectation values of the two Higgs doublets $\tan \beta$. For moderate $\tan \beta$ the mass eigenstates are similar to the gauge eigenstates ${ }^{3}$.

\subsubsection{R-Parity}

R-parity is a possible symmetry of the supersymmetric Lagrangian. It was first introduced to suppress lepton and baryon number violating processes at low energy scale and is defined by

$$
R=(-1)^{3 B+L+2 s}
$$

where $\mathrm{B}$ is the baryon number, $\mathrm{L}$ is the lepton number and $\mathrm{s}$ is the $\operatorname{spin}^{4}$. It can be seen that this combination ensures that

\footnotetext{
${ }^{3}$ In practice moderate $\tan \beta$ means $\tan \beta<10$.

${ }^{4} \mathrm{As} \mathrm{B}, \mathrm{L}, \mathrm{s}$ are quantized the definition $R=(-1)^{3(B-L)+2 s}$ is equivalent.
} 
- $R=1$ for Standard Model particles

- $R=-1$ for supersymmetric particles.

For this thesis it is assumed that - if supersymmetry is realized in nature - R-parity violating interaction vertices are not allowed and thus that R-parity is conserved. This has important consequences for the experimental signatures that can possibly be seen in detectors, as interactions always have to happen with an even number of supersymmetric particles and the lightest supersymmetric particle (LSP) is not able to decay and thus provides a good candidate for non-baryonic dark matter, if it is neither electrically nor color charged. As the LSP has $R=-1$ it can neither decay to supersymmetric particles, as this would violate energy conservation, nor to Standard Model particles, as this would violate Rparity conservation. If the lightest supersymmetric particle would have had electric or color charge it would be bound to nuclei and nuclei with unusual charge to mass ratio have not been observed[10]. The LSP escapes particle detectors unseen and carries away momentum. In hadron colliders the transverse momentum is conserved and a momentum imbalance can be a hint for a particle that was not seen in the detector. Momentum imbalance is an important signature for possibly supersymmetric events provided R-parity is conserved.

\subsubsection{Supersymmetry Breaking}

One of the axioms of the supersymmetry algebra[11] is

$$
\left[Q_{r}, P^{\mu}\right]=0
$$

where $Q_{r}$ is the supersymmetry operator and $P^{\mu}$ the four-momentum operator. Let $\mid$ boson $\rangle$ and $\mid$ fermion $\rangle$ be a Standard Model particle and its superpartner, so that $Q_{r} \mid$ boson $\rangle=$ $\mid$ fermion $\rangle$. It is thus

$$
\begin{aligned}
\left.P^{\mu} P_{\mu} Q_{r} \mid \text { boson }\right\rangle & \left.\left.=P^{\mu} P_{\mu} \mid \text { fermion }\right\rangle=m_{f}^{2} \mid \text { fermion }\right\rangle \\
& \left.\left.=Q_{r} P^{\mu} P_{\mu} \mid \text { boson }\right\rangle=m_{b}^{2} \mid \text { fermion }\right\rangle,
\end{aligned}
$$

where $m_{b}$ and $m_{f}$ are the masses of the boson and fermion respectively. If supersymmetry is not broken, it follows that $m_{f}=m_{b}$. The searches of collider experiments, however, have 
not found particles that are consistent with superpartners, so that — if supersymmetry is realized in nature - it has to be broken.

For phenomenological reasons, the supersymmetry breaking term should be soft enough to avoid reintroducing the hierarchy problem or destroying the unification of forces which is a good motivation for supersymmetry ${ }^{5}$. Without an underlying breaking model the most general supersymmetry breaking terms that assure these conditions are[7]

$$
\begin{aligned}
\mathcal{L}_{\mathrm{soft}}^{\mathrm{MSSM}} & =-\frac{1}{2}\left(M_{1} \tilde{B} \tilde{B}+M_{2} \tilde{W} \tilde{W}+M_{3} \tilde{g} \tilde{g}+\text { c.c. }\right) \\
& -\left(\tilde{\bar{u}}_{R} \mathbf{a}_{\mathbf{u}} \tilde{Q}_{L} H_{u}-\tilde{\bar{d}}_{R} \mathbf{a}_{\mathbf{d}} \tilde{Q}_{L} H_{d}-\tilde{\bar{e}}_{R} \mathbf{a}_{\mathbf{e}} \tilde{L}_{L} H_{d}+\text { c.c. }\right) \\
& -\tilde{Q}_{L}^{\dagger} \mathbf{m}_{\mathbf{Q}}^{2} \tilde{Q}_{L}-\tilde{L}_{L}^{\dagger} \mathbf{m}_{\mathbf{L}}^{\mathbf{2}} \tilde{L}_{L}-\tilde{\bar{u}}_{R} \mathbf{m}_{\overline{\mathbf{u}}}^{\mathbf{2}} \tilde{\bar{u}}_{R}^{\dagger}-\tilde{\bar{d}}_{R} \mathbf{m}_{\overline{\mathbf{d}}}^{\mathbf{2}} \tilde{\bar{d}}_{R}^{\dagger}-\tilde{\bar{e}}_{R} \mathbf{m}_{\overline{\mathbf{e}}}^{\mathbf{2}} \tilde{\bar{e}}_{R}^{\dagger} \\
& -m_{H_{u}}^{2} H_{u}^{*} H_{u}-m_{H_{d}}^{2} H_{d}^{*} H_{d}-\left(b H_{u} H_{d}+\text { c.c. }\right)
\end{aligned}
$$

where $M_{i}, i=1,2,3$ are the mass terms for Bino, Wino and gluino; $\mathbf{a}_{\mathbf{i}}$ are the scalar trilinear couplings; $m_{i}^{2}, i=L, Q, \bar{u}, \bar{d}, \bar{e}$ the slepton and squark mass terms and $m_{H_{u}}^{2}, m_{H_{d}}^{2}$ and $b$ the Higgs mass and mixing terms.

The way supersymmetry is broken affects the phenomenology of the specific supersymmetric model and most supersymmetric models are named after the breaking mechanism, e.g.

- gravity mediated supersymmetry breaking

- gauge mediated supersymmetry breaking.

Many theories of supersymmetry have at least two sectors: A visible sector, consisting of the supersymmetric particles which have been introduced earlier, and a hidden sector, consisting of particles which have no or very weak direct tree-level interactions with particles from the visible sector. Supersymmetry breaking is usually assumed to occur in the hidden sector and is transfered to the visible sector. Often this involves a third so-called messenger sector. The supersymmetry breaking mediated by gravity happens at the Planck scale

$$
M_{P}=\sqrt{\frac{\hbar c}{G_{N}}} \approx 1.22 \cdot 10^{28} \mathrm{eV}
$$

\footnotetext{
${ }^{5}$ See section 2.2.6 for details on the hierarchy problem and the unification of forces.
} 
and will be described in more detail in section 2.2.4. Gauge mediated supersymmetry breaking happens at lower scales at the order of several $\mathrm{TeV}$; the symmetry breaking is mediated by electroweak and QCD gauge interactions.

\subsection{4 mSUGRA}

Minimal supergravity grand unification is a proposed model where supersymmetry is a local symmetry. It is called supergravity since in order to achieve local supersymmetry it must include gravity as a fourth interaction in the calculations. In mSUGRA, non-renormalizable interaction terms between the hidden and the visible sector exist, but they are suppressed by $\frac{1}{M_{P}^{x}}$, where $x>1$. mSUGRA assumes that at the GUT scale ${ }^{6}$

$$
\begin{aligned}
m_{0}^{2} \mathbf{1} & :=\mathbf{m}_{\mathbf{Q}}^{\mathbf{2}}=\mathbf{m}_{\overline{\mathbf{u}}}^{\mathbf{2}}=\mathbf{m}_{\overline{\mathbf{d}}}^{\mathbf{2}}=\mathbf{m}_{\mathbf{L}}^{\mathbf{2}}=\mathbf{m}_{\overline{\mathbf{e}}}^{\mathbf{2}} \\
m_{0}^{2} & =m_{H_{u}}^{2}=m_{H_{d}}^{2}, \\
m_{1 / 2} & :=M_{1}=M_{2}=M_{3} \\
\mathbf{a}_{\mathbf{i}} & =A_{0} \mathbf{y}_{\mathbf{i}}, i=u, d, e \\
b & =B_{0} \mu .
\end{aligned}
$$

A realization of mSUGRA is fully defined by the parameters

$$
m_{0}, m_{1 / 2}, A_{0}, b, \mu \text {. }
$$

The masses and parameters at the weak scale can be obtained by using the renormalization group equation (RGE)[12]. Following [9] two relations for $b$ and $\mu$ can be derived by minimizing the Higgs potential and by using Eqn. 2.10

$$
\begin{aligned}
b & =\frac{\left(m_{H_{d}}^{2}-m_{H_{u}}^{2}\right) \tan 2 \beta+m_{Z}^{2} \sin 2 \beta}{2} \\
\mu^{2} & =\frac{m_{H_{u}}^{2} \sin ^{2} \beta-m_{H_{d}}^{2} \cos ^{2} \beta}{\cos 2 \beta}-\frac{m_{Z}^{2}}{2} .
\end{aligned}
$$

It is possible to determine $\mu$ and $b$ from $\tan \beta$ and the sign of $\mu$ as the mass of the $\mathrm{Z}$ boson $m_{Z}$ is well measured and the masses $m_{H_{u}}$ and $m_{H_{d}}$ are determined by $m_{0}$ and the

\footnotetext{
${ }^{6}$ The GUT scale is defined as the energy scale, where the couplings for the electromagnetic, weak and strong force unite. It is $m_{G U T} \approx 2 \cdot 10^{16} \mathrm{GeV}[12]$.
} 


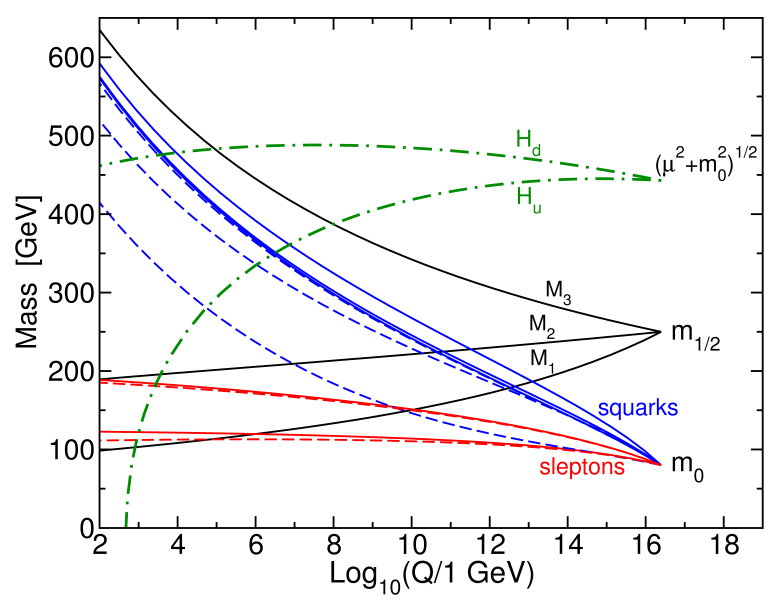

Figure 2.1: Evolution of different supersymmetric particle masses as a function of the renormalization scale $Q$ according to the renormalization group equation[7]. The gaugino and scalar masses are assumed to unite at the GUT scale in mSUGRA-like conditions; the negative mass of $H_{u}$ leads to a natural breaking of the electroweak symmetry.

renormalization group equation. The set of parameters given in Eqn. 2.34 is thus equivalent to the canonical set of parameters

$$
m_{0}, m_{1 / 2}, \tan \beta, A_{0}, \operatorname{sgn} \mu
$$

where

- $m_{0}$ is the common scalar mass at the GUT scale,

- $m_{1 / 2}$ is the common gaugino mass at the GUT scale,

- $\tan \beta=v_{u} / v_{d}$ is the ratio of the vacuum expectation values of the two Higgs doublets,

- $A_{0}$ is the common trilinear coupling at the GUT scale and

- $\operatorname{sgn} \mu$ is the sign of the Higgs mixing parameter.

In this thesis a benchmark point, henceforth called benchmark point $\mathrm{BP} 1$, with $m_{0}=$ $60 \mathrm{GeV} / \mathrm{c}^{2}, m_{1 / 2}=190 \mathrm{GeV} / \mathrm{c}^{2}, \tan \beta=3, A_{0}=0 \mathrm{GeV}, \mu>0$ is defined to evaluate the sensitivity of the analysis. The benchmark point was chosen to maximize the sensitivity for a trilepton signature at reasonable chargino and neutralino masses. 


\subsubsection{Neutralinos and Charginos}

It was already noted in section 2.2.1 that the supersymmetric partners of the Higgs and gauge bosons, the Higgsinos $\tilde{H}_{u}^{+}, \tilde{H}_{d}^{-}, \tilde{H}_{u}^{0}, \tilde{H}_{d}^{0}$, the Bino $\tilde{B}^{0}$ and the Winos $\tilde{W}^{0}, \tilde{W}^{ \pm}$are gauge eigenstates, but not mass eigenstates. The charged particles mix to form the charginos $\tilde{\chi}_{i}^{ \pm}, i=1,2$, while the neutral particles mix to form the neutralinos $\tilde{\chi}_{i}^{0} i=1,2,3,4$.

In the basis $\left(\tilde{B}^{0}, \tilde{W}^{0}, \tilde{H}_{d}^{0}, \tilde{H}_{u}^{0}\right)$ the neutralino mass matrix is[7]

$$
\left(\begin{array}{cccc}
M_{1} & 0 & -g_{Y} v_{d} / \sqrt{2} & g_{Y} v_{u} / \sqrt{2} \\
0 & M_{2} & g_{2} v_{d} / \sqrt{2} & -g_{2} v_{u} / \sqrt{2} \\
-g_{Y} v_{d} / \sqrt{2} & g_{2} v_{d} / \sqrt{2} & 0 & -\mu \\
g_{Y} v_{u} / \sqrt{2} & -g_{2} v_{u} / \sqrt{2} & -\mu & 0
\end{array}\right),
$$

where $M_{1}$ and $M_{2}$ are the MSSM parameters for the Bino and Wino mass and $\mu$ is the Higgs mixing parameter from the term $\propto \mu H_{u} H_{d}$. The terms in the off-diagonal $2 \times 2$ submatrices are the trilinear couplings between gaugino, Higgs and Higgsino. Using Eqn. 2.10, $v_{d}=$ $v \cos \beta, v_{u}=v \sin \beta$ and the definition of the weak mixing angle $\cos \theta_{W}=m_{W} / m_{Z}$ this can be recast into

$$
\left(\begin{array}{cccc}
M_{1} & 0 & -\cos \beta \sin \theta_{W} m_{Z} & \sin \beta \sin \theta_{W} m_{Z} \\
0 & M_{2} & \cos \beta \sin \theta_{W} m_{Z} & -\sin \beta \sin \theta_{W} m_{Z} \\
-\cos \beta \sin \theta_{W} m_{Z} & \cos \beta \cos \theta_{W} m_{Z} & 0 & -\mu \\
\sin \beta \sin \theta_{W} m_{Z} & -\sin \beta \cos \theta_{W} m_{Z} & -\mu & 0
\end{array}\right) .
$$

In the case of the the charged Higgsinos and Winos and a gauge eigenstate basis of the form $\left(\tilde{W}^{+}, \tilde{H}_{u}^{+}, \tilde{W}^{-}, \tilde{H}_{d}^{-}\right)[1]$ the mass matrix for the charginos can be written as

$$
\begin{aligned}
M_{\tilde{\chi}^{ \pm}} & =\left(\begin{array}{cc}
0 & X^{T} \\
X & 0
\end{array}\right), \text { where } \\
X & =\left(\begin{array}{cc}
M_{2} & g_{2} v_{u} \\
g_{2} v_{d} & \mu
\end{array}\right)=\left(\begin{array}{cc}
M_{2} & \sqrt{2} \sin \beta m_{W} \\
\sqrt{2} \cos \beta m_{W} & \mu
\end{array}\right),
\end{aligned}
$$

so that the mass term in the Lagrangian can be written as

$$
\mathcal{L}_{\text {chargino mass }}=-\frac{1}{2}\left(\psi^{ \pm}\right)^{T} M_{\tilde{\chi}^{ \pm}} \Psi^{ \pm}+\text {c.c. } .
$$


If the relation between the mass and the gauge eigenstates is written as

$$
\left(\begin{array}{c}
\tilde{\chi}_{1}^{+} \\
\tilde{\chi}_{2}^{+}
\end{array}\right)=V\left(\begin{array}{c}
\tilde{W}^{+} \\
\tilde{H}_{u}^{+}
\end{array}\right) \quad ; \quad\left(\begin{array}{c}
\tilde{\chi}_{1}^{-} \\
\tilde{\chi}_{2}^{-}
\end{array}\right)=U\left(\begin{array}{c}
\tilde{W}^{-} \\
\tilde{H}_{d}^{-}
\end{array}\right),
$$

where $U$ and $V$ are unitary matrices, the mass matrix can be diagonalized by

$$
U^{*} X V^{-1}=\left(\begin{array}{cc}
\tilde{\chi}_{1}^{ \pm} & 0 \\
0 & \tilde{\chi}_{2}^{ \pm}
\end{array}\right)
$$

and the masses at tree-level are

$$
\begin{aligned}
& m_{\chi_{1}^{ \pm}}^{2}, m_{\chi_{2}^{ \pm}}^{2}= \\
& \left.\frac{1}{2}\left[\mid M_{2}\right]^{2}+|\mu|^{2}+2 m_{W}^{2} \mp \sqrt{\left(\left|M_{2}\right|^{2}+|\mu|^{2}+2 m_{W}^{2}\right)^{2}-4\left|\mu M_{2}-m_{W}^{2} \sin 2 \beta\right|^{2}}\right] .
\end{aligned}
$$

If the gaugino masses and the gauge couplings are assumed to unify at the GUT scale, it is[12]

$$
\frac{M_{3}}{g_{s}^{2}}=\frac{M_{2}}{g_{2}^{2}}=\frac{M_{1}}{5 / 3 g_{Y}^{2}}=\frac{m_{1 / 2}}{g^{2}\left(M_{G U T}\right)}
$$

invariant under application of the renormalization group equation ${ }^{7}$. Using Eqn. 2.10 this implies that

$$
\frac{M_{1}\left(m_{Z}\right)}{M_{2}\left(m_{Z}\right)}=\frac{5}{3} \tan ^{2} \theta_{W} \approx \frac{1}{2}
$$

In the limit of $|\mu| \gg\left|M_{i}\right| \gg m_{Z}, i=1,2$ the lightest neutralino $\tilde{\chi}_{1}^{0}$ is mostly the Bino; the next to lightest neutralino $\tilde{\chi}_{2}^{0}$ is mostly the neutral Wino and the neutralinos $\tilde{\chi}_{i}^{0}$, $i=3,4$, are mostly neutral Higgsinos. The lighter chargino $\tilde{\chi}_{1}^{ \pm}$is mostly the charged Wino, whereas the heavier chargino is mostly the charged Higgsino. Furthermore

$$
m_{\tilde{\chi}_{1}^{ \pm}} \approx m_{\tilde{\chi}_{2}^{0}} \approx 2 \cdot m_{\tilde{\chi}_{1}^{0}}, \quad|\mu| \approx m_{\tilde{\chi}_{3}^{0}} \approx m_{\tilde{\chi}_{4}^{0}} \approx m_{\tilde{\chi}_{2}^{ \pm}} \gg m_{\tilde{\chi}_{1}^{ \pm}}
$$

following Eqn. 2.46.

In the limit of $|\mu| \ll\left|M_{i}\right|, i=1,2$, the neutralinos $\tilde{\chi}_{i}^{0}, i=1,2$, are mostly the neutral Higgsinos; the neutralinos $\tilde{\chi}_{i}^{0}, i=3,4$, are the Bino and neutral Wino whereas the lighter

\footnotetext{
${ }^{7}$ The factor $5 / 3$ comes from the difference between the GUT normalization and the usual SM normalization of the hypercharge generator[12].
} 
chargino $\tilde{\chi}_{1}^{ \pm}$is mostly the charged Higgsino and the heavier chargino $\tilde{\chi}_{2}^{ \pm}$mostly the charged Wino. Following Eqn. 2.46

$$
m_{\tilde{\chi}_{1}^{ \pm}} \approx m_{\tilde{\chi}_{1}^{0}} \approx m_{\tilde{\chi}_{2}^{0}} \approx|\mu|, \quad 2 \cdot m_{\tilde{\chi}_{3}^{0}} \approx m_{\tilde{\chi}_{4}^{0}} \approx m_{\tilde{\chi}_{2}^{ \pm}}
$$

The relevant couplings of the chargino and neutralino components are listed in Fig. 2.2. It has to be noted that the charged Wino $\tilde{W}^{ \pm}$couples only to left-handed sleptons and that the Higgsino $\tilde{H}$ has a Yukawa coupling to third generation particles. The actual decays of the neutralinos and charginos depend strongly on the mass spectrum and the kinematically allowed decay channels. The relevant possible two-body decay channels of the neutralino are

$$
\begin{aligned}
& \tilde{\chi}_{i}^{0} \rightarrow \tilde{\chi}_{j}^{0} Z^{0}, \\
& \tilde{\chi}_{i}^{0} \rightarrow \tilde{\chi}_{j}^{ \pm} W^{ \pm}, \\
& \tilde{\chi}_{i}^{0} \rightarrow \tilde{l}^{ \pm} l^{\mp}, \\
& \tilde{\chi}_{i}^{0} \rightarrow \tilde{\nu}_{l} \nu_{l}, \\
& \tilde{\chi}_{i}^{0} \rightarrow \tilde{\chi}_{j}^{0} h^{0},
\end{aligned}
$$

where $l=e, \mu, \tau$. Sleptons and sneutrinos subsequently mostly decay according to

$$
\begin{gathered}
\tilde{\nu}_{l} \rightarrow \tilde{\chi}_{i}^{0} \nu_{l}, \\
\tilde{l}^{ \pm} \rightarrow \tilde{\chi}_{i}^{0} l^{ \pm} .
\end{gathered}
$$

Decays of the neutralinos into $\tilde{\chi}_{j}^{0} A^{0}, \tilde{\chi}_{j}^{0} H^{0}, \tilde{\chi}_{j}^{\mp} H^{ \pm}$and $\tilde{q} q$ are possible for the right choice of the supersymmetric parameters, but not dominant for the parameter space considered in this thesis. If neither of the two-body decay channels is kinematically allowed, usually a three-body decay via off-shell $Z^{0 *}$ boson happens according to

$$
\tilde{\chi}_{i}^{0} \rightarrow \tilde{\chi}_{j}^{0} Z^{0 *} \rightarrow \tilde{\chi}_{j}^{0}\left\{\begin{array}{l}
l^{+} l^{-} \\
q \bar{q} \\
\nu_{l} \bar{\nu}_{l}
\end{array}\right.
$$




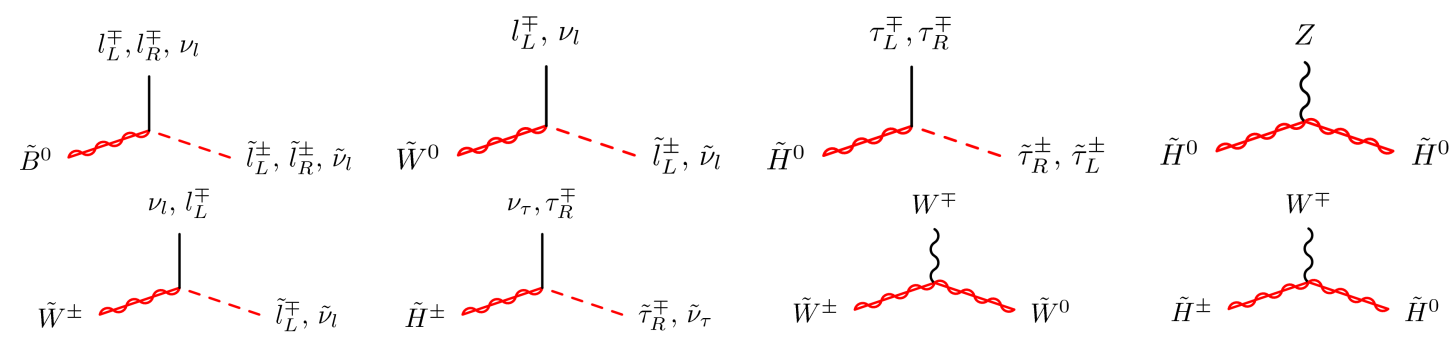

Figure 2.2: Relevant allowed vertices for the decay of chargino and neutralino into leptons

The decays of the charginos are similar with the decay channels

$$
\begin{aligned}
& \tilde{\chi}_{i}^{ \pm} \rightarrow \tilde{\chi}_{j}^{0} W^{ \pm}, \\
& \tilde{\chi}_{i}^{ \pm} \rightarrow \tilde{\chi}_{j}^{ \pm} Z^{0}, \\
& \tilde{\chi}_{i}^{ \pm} \rightarrow \tilde{l}^{ \pm} \nu_{l}, \\
& \tilde{\chi}_{i}^{ \pm} \rightarrow \tilde{\nu}_{l} l^{ \pm},
\end{aligned}
$$

where the slepton and sneutrino subsequently decay according to Eqns. 2.54 and 2.55. Decays into $\tilde{\chi}_{j}^{ \pm} h^{0}, \tilde{\chi}_{j}^{ \pm} A^{0}, \tilde{\chi}_{j}^{ \pm} H^{0}, \tilde{\chi}_{j}^{0} H^{ \pm}$and $\tilde{q}_{1} \bar{q}_{2}$ are possible, but not dominant for the parameter space considered in this thesis. If none of the two-body decay channels is kinematically allowed usually a three-body decay via off-shell $W^{ \pm *}$ boson happens according to

$$
\tilde{\chi}_{i}^{ \pm} \rightarrow \tilde{\chi}_{j}^{0} W^{ \pm *} \rightarrow \tilde{\chi}_{j}^{0}\left\{\begin{array}{c}
l^{ \pm} \nu_{l} \\
q_{1} \bar{q}_{2}
\end{array}\right.
$$

\subsubsection{Supersymmetry and the Limitations of the Standard Model}

High energy physics experiments have tested the Standard Model of Particle Physics in collisions up to a center-of-mass energy of $1.96 \mathrm{TeV}$, which can currently be reached at the Tevatron accelerator at Fermilab. The predictions of the Standard Model agree very well with the experimental results and additional structure has not yet been seen. However, the Standard Model of Particle Physics has at least 18 free parameters ${ }^{8}$ and an underlying, more fundamental theory is expected. Furthermore there are several open questions that are not addressed by the Standard Model.

\footnotetext{
${ }^{8}$ It should be noted that general MSSM has 110 free parameters and thus might not be considered a fundamental theory.
} 


\section{The Hierarchy Problem}

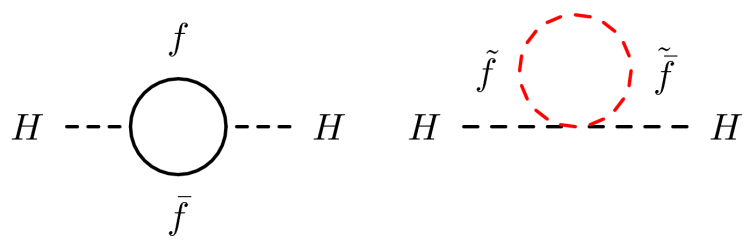

Figure 2.3: Fermionic and sfermionic loop contributions to the scalar Higgs mass

In Eqn. 2.4 a term proportional to $\left(\phi^{\dagger} \phi\right)^{2}$ was introduced into the Lagrangian to ensure electroweak symmetry breaking. If the mass of the Higgs boson is calculated, 1-loop level contributions from fermionic and scalar loops have to be considered. An example of a fermionic loop can be found in Fig. 2.3. For an interaction term of the form $-\lambda_{f} H \bar{f} f$ the contribution of the fermionic loop to the Higgs mass in Landau notation is

$$
\Delta m_{H}^{2}=-\frac{\left|\lambda_{f}\right|^{2}}{8 \pi^{2}} \Lambda^{2}+\mathcal{O}\left(\ln \left(\frac{\Lambda}{m_{f}}\right)\right)[7],
$$

where $\Lambda$ is a cutoff parameter for the applicability of the Standard Model and $m_{f}$ is the mass of the fermion in the loop. The bare mass of the Higgs boson can be obtained from Eqn. 2.4 as

$$
m_{0}^{2}=2 \mu^{2}=-2 \lambda v^{2}
$$

so that the Higgs mass is $m_{H}^{2}=m_{0}^{2}+\sum_{\text {all loops }} \Delta m_{H}^{2}$. Based on data collected at the LEP experiment the Higgs mass was constrained at $95 \%$ confidence level to

$$
114.4 \mathrm{GeV} / \mathrm{c}^{2}<m_{H}<193 \mathrm{GeV} / \mathrm{c}^{2}
$$

in $[13,14]$. As the cutoff for new physics $\Lambda$ is very large in order to get a Higgs mass in this allowed interval, a cancellation of the mass corrections and the bare Higgs mass is needed. In supersymmetric models a cancellation happens naturally as the contributions by Standard Model particles are canceled by the contributions of supersymmetric particles. Without supersymmetric particles the cancellation of the bare mass and the corrections is regarded unnatural. This is known as the hierarchy or the fine-tuning problem. 


\section{Unification of Forces}

In quantum field theory coupling constants are defined by

$$
\alpha=\frac{g_{x}^{2}}{2 \pi}
$$

where $g_{x}$ is an energy dependent coupling parameter. As the values of the coupling parameters are known to high precision at the scale $Q=M_{Z}[1]$ their value at arbitrary scale can be obtained by application of the renormalization group equation. The renormalization group equation at 1-loop order is[7]

$$
\frac{d}{d t} g_{a}=\frac{1}{16 \pi^{2}} b_{a} g_{a}^{3}, \quad \text { where } \quad b_{1,2,3}= \begin{cases}(41 / 10, / 19 / 6,-7) & \text { Standard Model } \\ (33 / 5,1,-3) & \text { MSSM }\end{cases}
$$

$t=\ln \left(Q / Q_{0}\right)$ and $Q_{0}$ is a high energy scale, where different contributions to the Lagrangian are assumed. The evolution of the gauge couplings as a function of the scale $Q$ is shown in Fig. 2.4. It can be seen, that the couplings do not fully unite in the SM evolution, whereas in the MSSM evolution supersymmetric loop contributions have to be considered and the couplings unite at the precision of the figure.

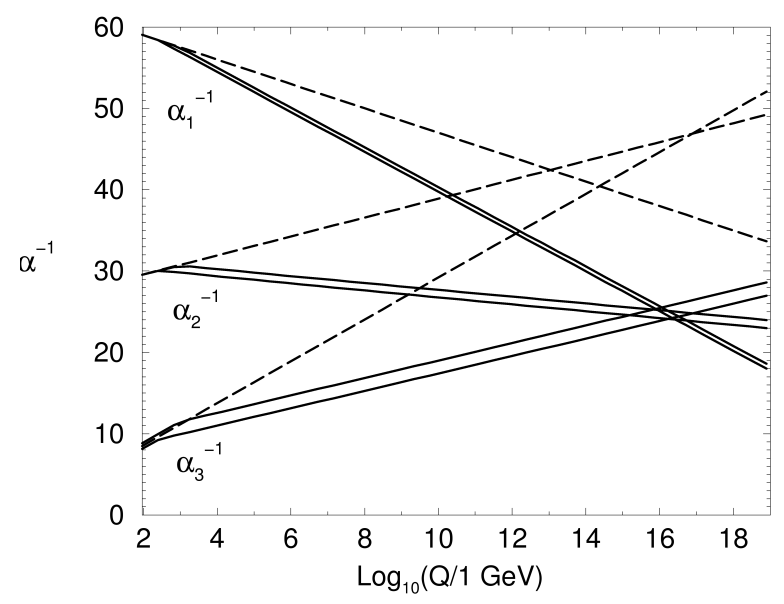

Figure 2.4: Inverse gauge coupling evolution as a function of the renormalization scale $Q$ according to the renormalization group equation for the Standard Model (dashed lines) and the MSSM (solid lines). In the MSSM case, the sparticle mass thresholds are varied between $250 \mathrm{GeV}$ and $1 \mathrm{TeV}$, and $\alpha_{3}\left(m_{Z}\right)$ between 0.113 and 0.123 . Two-loop effects are included[7]. 


\section{Dark Matter and Dark Energy}

The Friedmann equation is[15]

$$
H^{2}=\frac{\dot{R}^{2}}{R^{2}}=\frac{8 \pi G_{N}}{3} \rho-\frac{k c^{2}}{R^{2}},
$$

where $H$ is the Hubble constant, $R$ an arbitrary length, which is used as a measure for the metric of the universe, $G_{N}$ Newton's gravitational constant and k a constant that is -1 for an open, 0 for a flat and 1 for a geometrically closed universe. The Friedmann equation describes the time evolution and the geometry of the universe and is strongly dependent on the density of the universe $\rho$. For the critical density

$$
\rho_{c}=\frac{3 H^{2}}{8 \pi G_{N}}
$$

the universe is flat. The density parameter of the universe is defined by

$$
\Omega_{m}=\frac{\rho}{\rho_{c}}
$$

$\Omega_{b}$, defined by $\Omega_{b}=\rho_{b} / \rho_{c}$, where $\rho_{b}$ is the baryonic density, is called the baryonic density parameter; $\Omega_{c}$ is the cold dark matter density parameter, so that $\Omega_{m}=\Omega_{b}+\Omega_{c}$, where $\Omega_{m}$ is the density parameter for all matter. Dark matter is matter that does not take part in the electromagnetic and strong interactions, but can be observed by its gravitational interaction. The Friedmann equation in the form of Eqn. 2.67 describes a decelerating universe; Einstein introduced the term $-\frac{1}{3} \Lambda c^{2}$ where $\Lambda$ is the cosmological constant. The cosmological constant can be interpreted as acceleration due to dark energy. The density parameter is given by $\Omega_{\Lambda}=\frac{\Lambda}{3 H_{0}^{2}}$.

The Wilkinson Microwave Anisotropy Probe (WMAP) is a satellite which recorded the cosmic microwave background. A six parameter cosmological model is fit to the data and together with measurements from Type Ia supernovae the following results can be obtained[16]:

$$
\begin{aligned}
& \Omega_{b}=0.0462 \pm 0.0015 \\
& \Omega_{c}=0.233 \pm 0.013 \\
& \Omega_{\Lambda}=0.721 \pm 0.015
\end{aligned}
$$


From Eqns. 2.70 to 2.72 it can be derived that baryonic matter makes up less than $5 \%$ of the energy and less than $20 \%$ of the matter in the universe.

It is still unknown what dark matter is made of. The Standard Model does not offer particles that might make up dark matter. In the case of conserved R-parity the lightest supersymmetric particle is stable and in the case of a neutral LSP it can serve as a dark matter candidate. In big regions of the MSSM parameter space the lightest neutralino is an uncharged and color neutral LSP and is thus able to make up dark matter.

A review on reasons to judge if supersymmetry might be the answer to outstanding problems of the Standard Model can be found in [17]. 


\section{Chapter 3}

\section{The Experimental Apparatus}

This analysis is using data collected by the Collider Detector at Fermilab in the configuration of Run II (CDF II) detector. The CDF detector is one of two multipurpose detectors that use $1.96 \mathrm{TeV}$ proton-antiproton collisions taking place in the Tevatron accelerator at the Fermi National Accelerator Laboratory (FNAL, Fermilab). Until the Large Hadron Collider is in operation Fermilab houses the world's most energetic operating particle accelerator.

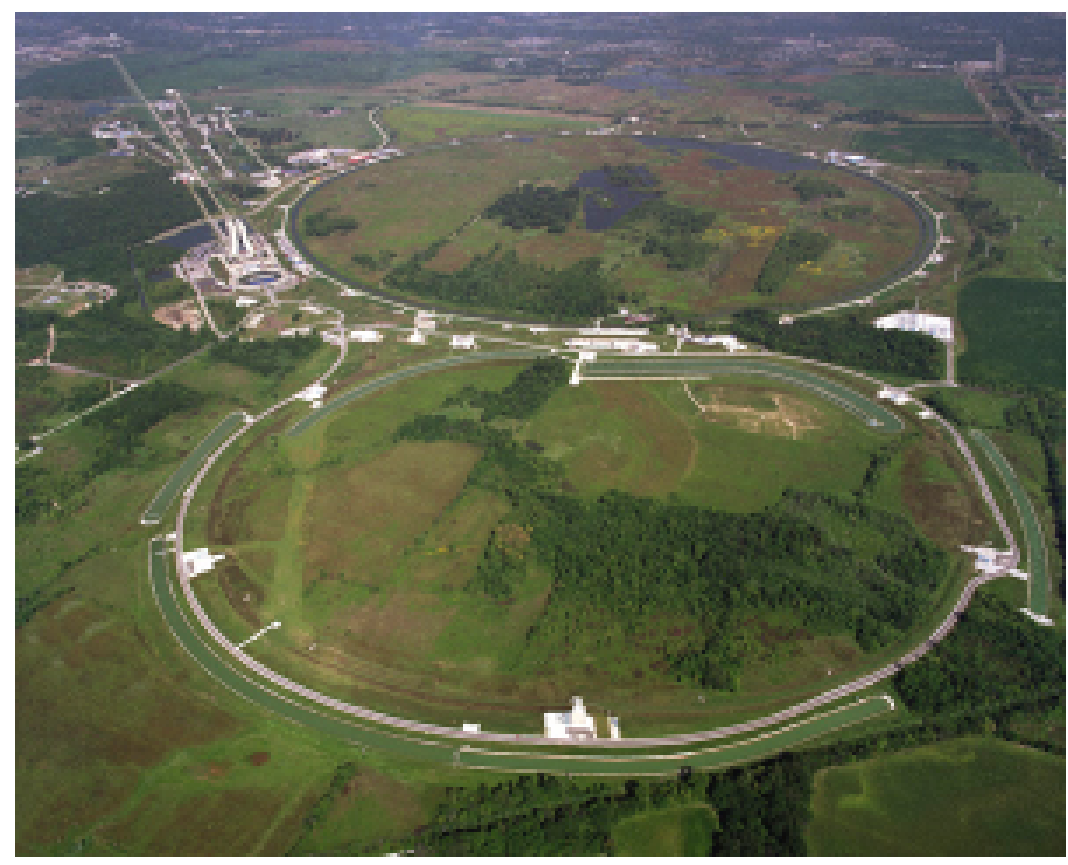

Figure 3.1: Aerial view of the Tevatron (top) and the Main Injector (bottom). Robert Rathbun Wilson Hall ("The High Rise"), the main office and laboratory building, is in the top left. The CDF Detector Hall is the red building next to the Tevatron ring. 


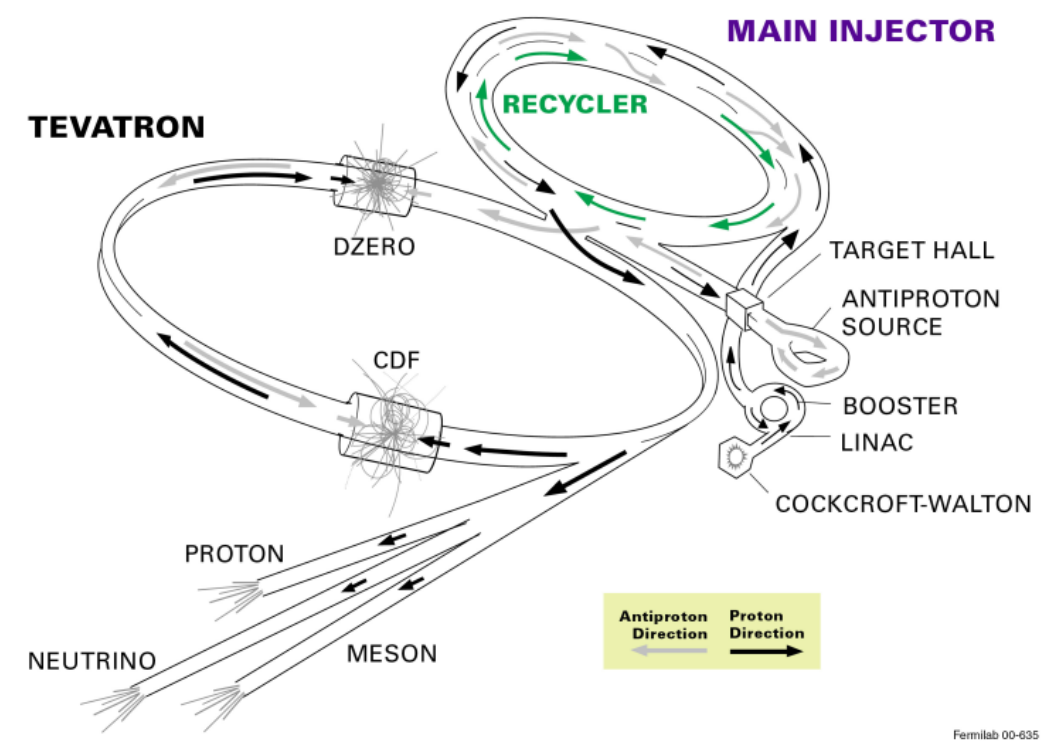

Figure 3.2: Artistic illustration of the accelerator chain at Fermilab.

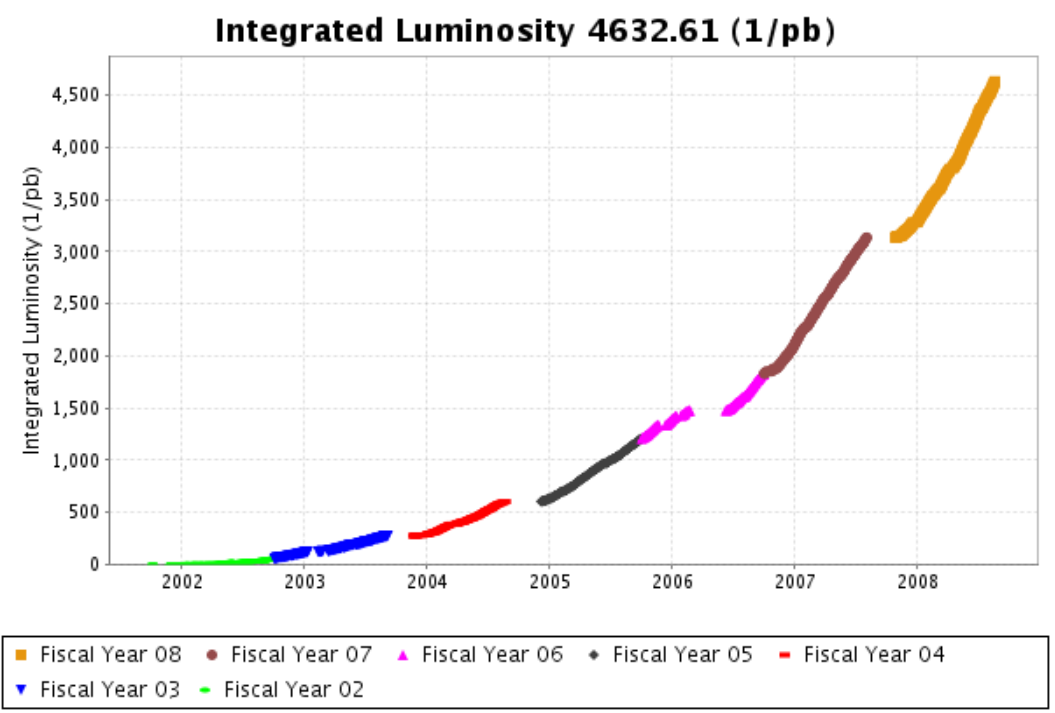

Figure 3.3: Integrated luminosity delivered by the Tevatron accelerator as of August 20, $2008[18]$. 


\subsection{The Tevatron and its Preaccelerators}

At Fermilab hydrogen is ionized and accelerated in a magnetron to $25 \mathrm{keV}$. The resulting $\mathrm{H}^{-}$ ions are passed to a Cockcroft-Walton preaccelerator, a voltage multiplier, which by using a constant voltage accelerates the $\mathrm{H}^{-}$ions to $750 \mathrm{keV}$ and inserts them into the Linac, an approximately $150 \mathrm{~m}$ long linear accelerator. The Linac uses oscillating electric fields to reach an energy of $400 \mathrm{MeV}$. At the end of the Linac the $\mathrm{H}^{-}$ions beam crosses a carbon foil and electrons are stripped off, so that a proton beam can be separated. The proton beam is passed into the Booster, the first circular accelerator. Having a circumference of $475 \mathrm{~m}$ it accelerates the protons to an energy of $8 \mathrm{GeV}$. From the Booster the proton beam is inserted into the Main Injector, which fulfills three functions: It can accelerate protons to an energy of $150 \mathrm{GeV}$ and insert them into the Tevatron; it can accelerate protons to an energy of $120 \mathrm{GeV}$ and shoot them into a nickel target to produce antiprotons and it can accelerate previously produced antiprotons to $150 \mathrm{GeV}$ and insert them into the Tevatron. When shot into a nickel target a $120 \mathrm{GeV}$ proton beam produces antiprotons with different energies and angles among a lot of other secondary particles. Antiprotons with an energy of approximately $8 \mathrm{GeV}$ are focused by a lithium lense and cooled and stacked in the Debuncher and the Accumulator. The production of antiprotons has always been one of the main limiting factors for the Tevatron operation. For this reason the Recycler ring was built in the tunnel of the Main Injector. The Recycler's role is to store antiprotons from the Accumulator and cool them further down to increase the antiproton stacking rate. Unlike the Accumulator the Recycler's magnet system can store antiprotons efficiently for a longer time. Additionally the Recycler was designed to store antiprotons that are left over from previous Tevatron runs, however, this operation mode is currently not used.

The Tevatron finally accelerates protons and antiprotons to an energy of approximately $1 \mathrm{TeV}$, so that a center-of-mass energy of $\sqrt{s}=1.96 \mathrm{TeV}$ can be reached in a protonantiproton collision. In the Tevatron protons and antiprotons are ordered in 140 and 103 bunches respectively[19] and are accelerated separately, so that they collide in the two multi-purpose detectors CDF and D $\varnothing$ at the sector points B0 and D0. Every proton bunch consists of approximately $2.7 \times 10^{11}$ protons and every antiproton bunch of approximately 
$1.0 \times 10^{11}$ antiprotons at a length of $37 \mathrm{~cm}$. The bunches have a separation of $132 \mathrm{nsec}$. Integrated Luminosity is a measure for the number of collisions that have been delivered. The Tevatron currently delivers a peak luminosity of[18]

$$
\mathcal{L} \approx 3.2 \times 10^{32} \frac{1}{\mathrm{~cm}^{2} \mathrm{~s}}=3.2(\mu \mathrm{b})^{-1}
$$

which currently leads to a weekly integrated luminosity of

$$
\int_{\text {week }} \mathcal{L}(t) d t \approx 5.5 \times 10^{37} \frac{1}{\mathrm{~cm}^{2} \mathrm{~s}}=55(\mathrm{pb})^{-1} .
$$

\subsection{The Collider Detector at Fermilab}

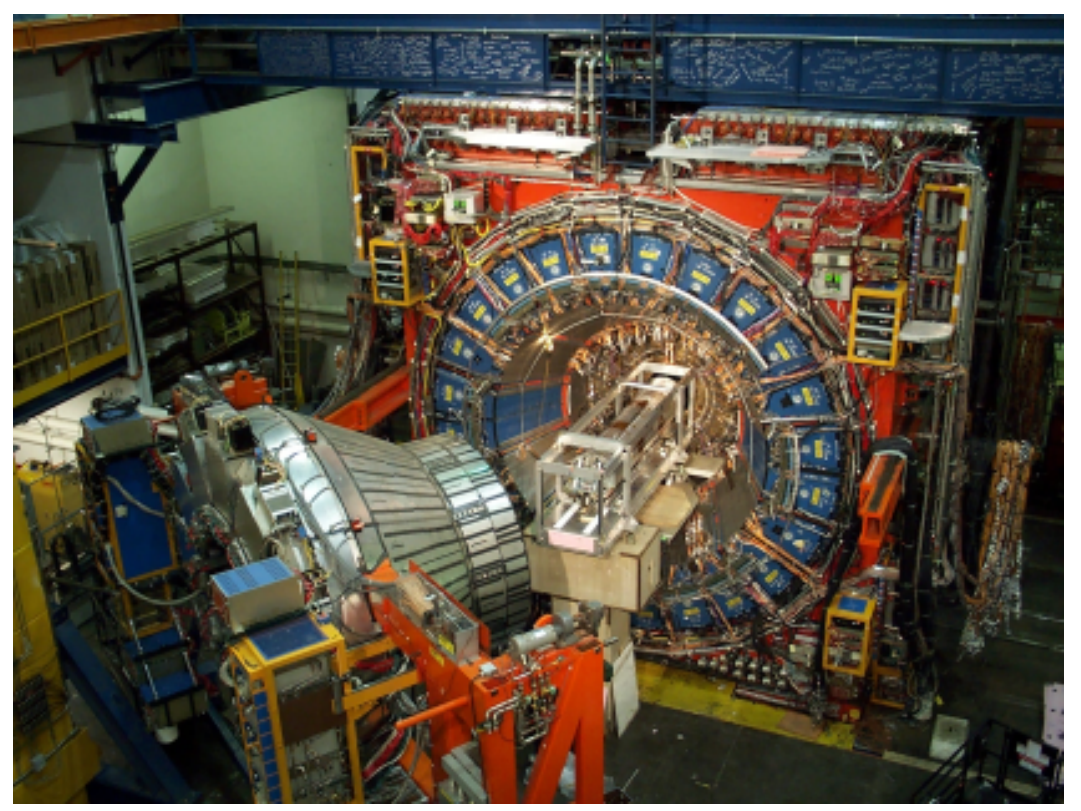

Figure 3.4: Photo of the Collider Detector at Fermilab with opened plug

The Collider Detector at Fermilab $\left(\mathrm{CDF} \mathrm{II}^{1}\right)[20,21]$ is one of two multipurpose detectors at the Tevatron. It is built symmetrically in azimuthal direction around the Tevatron beamline, is measuring approximately $12 \mathrm{~m} \times 12 \mathrm{~m} \times 12 \mathrm{~m}$ and weighing approximately 1000 metric tons. Figure 3.5 shows a schematic drawing of the CDF II detector. Starting at the Tevatron beamline the CDF II detector's main components are the tracking system, the solenoid, the electromagnetic and the hadronic calorimeters and the muon detector system.

\footnotetext{
${ }^{1}$ The Collider Detector at Fermilab was updated after the end of Run I. The name CDF II refers to the configuration of the detector in Run II.
} 
The solenoid generates a $1.4 \mathrm{~T}$ magnetic field, which bends the tracks of charged particles, so that their momentum can be determined in the tracker.

The CDF coordinate system is uniquely defined by the coordinates $(r, \theta, \phi)$ which is equivalent to $(r, \eta, \phi)$, where

the radius $r$ is measured from the center of the detector,

the polar angle $\theta$ is measured from the beamline direction,

the azimuthal angle $\phi$ is measured from the plane defined by the Tevatron ring and the pseudorapidity $\eta$ is defined by $\eta=-\ln \tan \frac{\theta}{2}$.

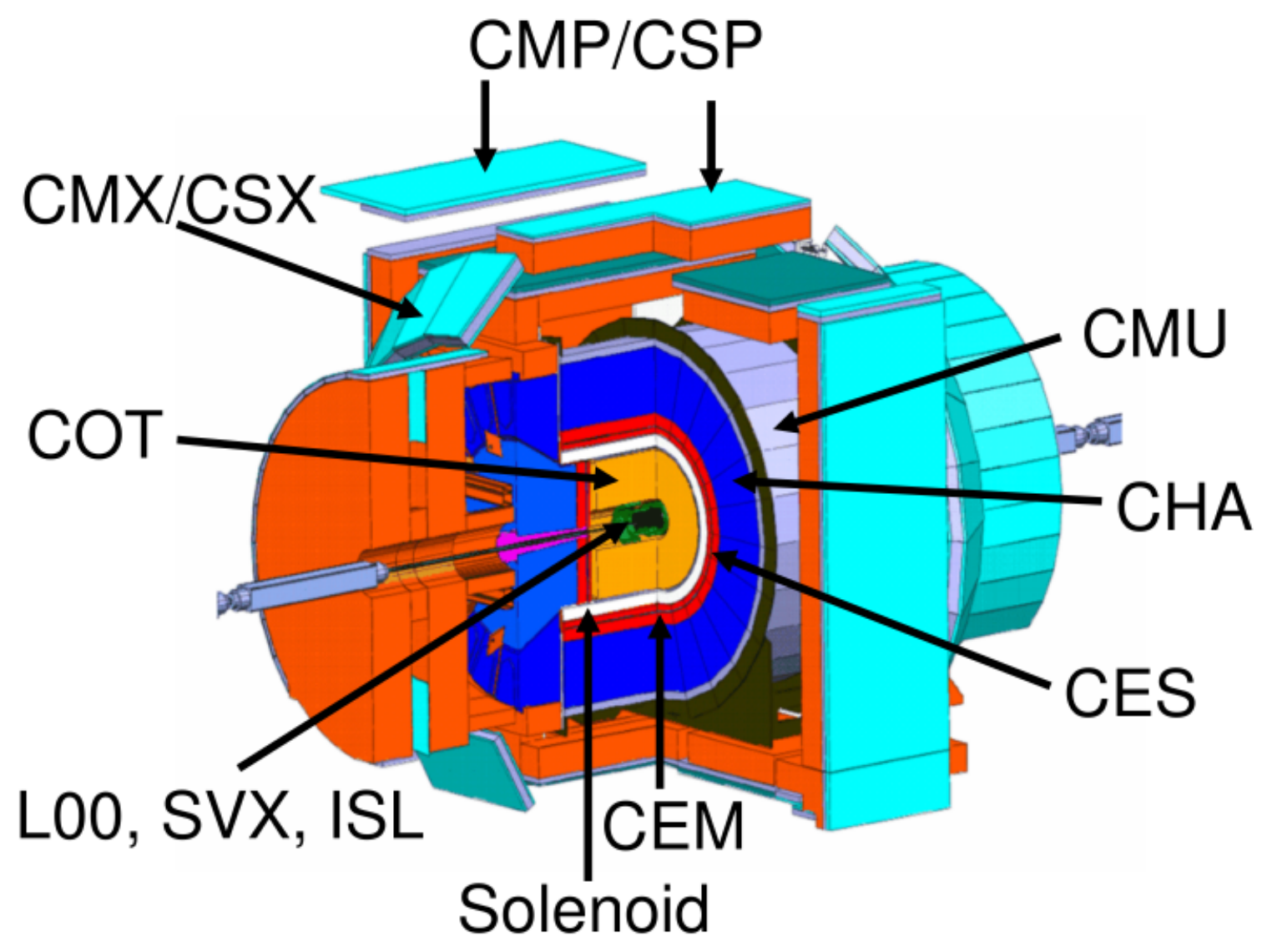

Figure 3.5: Parts of the Collider Detector at Fermilab (CDF). L00: Layer 00 silicon tracker, SVX: Silicon VerteX detector, ISL: Intermediate Silicon Layers, COT: Central Outer Tracker, CEM: Central ElectroMagnetic calorimeter, CES: Central Electromagnetic Strip chamber, CHA: Central HAdronic calorimeter, CMU: Central MUon detector, CMP: Central Muon uPgrade detector, CSP: Central muon Scintillator uPgrade, CMX: Central Muon eXtension detector, CSX: Central muon eXtension Scintillators 


\subsubsection{The Tracking System}

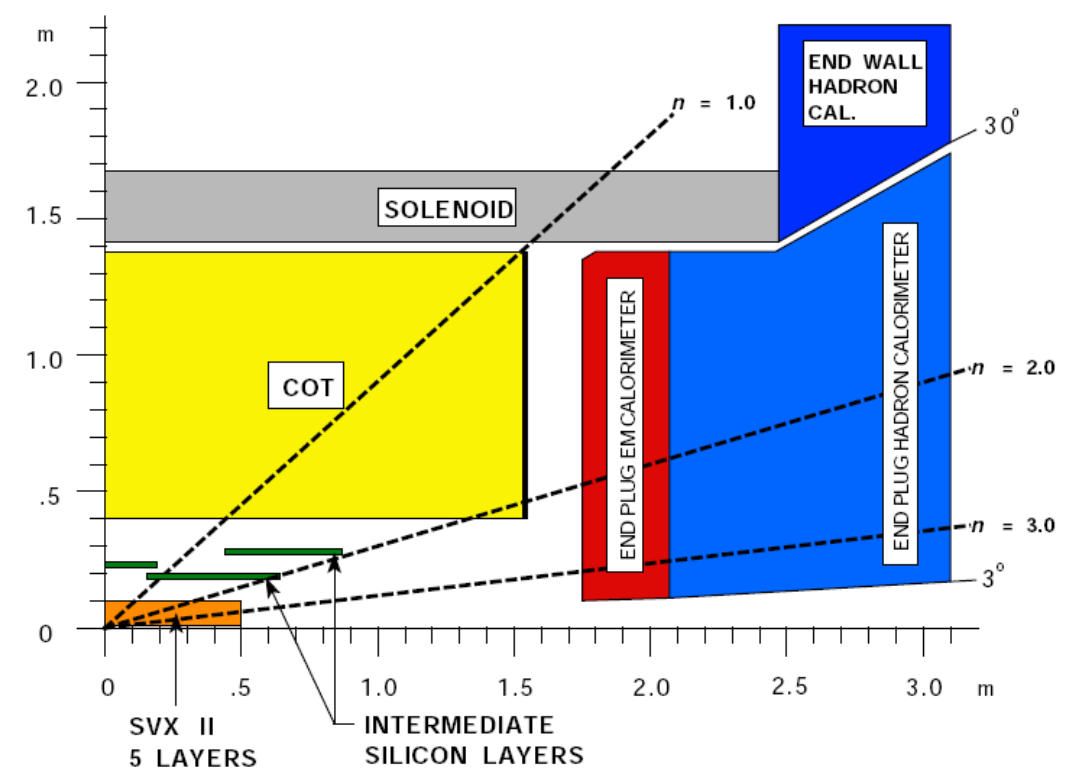

Figure 3.6: Longitudinal view of the CDF II tracking system[20] and parts of the calorimeter. The Layer 00 tracking system is the grey line below the SVX.

The CDF Tracking system is shown in Fig. 3.6 and consists of the silicon detectors Layer 00, the Silicon Vertex Detector, the Intermediate Silicon Layers and a proportional wire chamber, the Central Outer Tracker.

\section{The Silicon Tracker}

In silicon detectors charged particles produce electron-hole pairs. If a voltage is applied, a current can be measured and, for several read-out channels, a particle track can be reconstructed. The Layer 00 tracking system is a single-sided silicon layer starting at $1.35 \mathrm{~cm}$ around the center of the beam pipe. Around the Layer 00 tracker the Silicon Vertex Detector (SVX) was built. It consists of 5 double-sided silicon layers placed from $2.44 \mathrm{~cm}$ to $10.6 \mathrm{~cm}$. Whereas single-sided silicon layers only have a one-dimensional resolution, doublesided silicon layers have two layers rotated by $90^{\circ}$ (for three of five layers) or $1.2^{\circ}$ (small angle stereo for two layers) towards each other to provide a two-dimensional resolution. In the region $|\eta|<1$ full 3D track reconstruction is possible, if tracks in the Silicon Vertex Detector and the Central Outer Tracker are matched. For $\eta>1$ the SVX can provide only 
$2 \mathrm{D}$ vertex reconstruction. The Intermediate Silicon Layers (ISL) are additional single silicon layers, which are placed at $22 \mathrm{~cm}$ for $|\eta|<1$ and at $20 \mathrm{~cm}$ and $28 \mathrm{~cm}$ around the beamline for $1<|\eta|<2$. The main design goals of the L00, the SVX and the ISL layers were to reach a precise impact parameter, secondary vertex and $z_{0}$ measurement. The impact parameter $d_{0}$ is the distance of the particle's track to the beamline in the $(r, \phi)$-plane; secondary vertex measurement is needed for B-tagging ability and $z_{0}$ is the distance from the intersection of the interpolation of the track and the beamline to the center of the detector. The silicon tracking system can provide a hit resolution of $11 \mu \mathrm{m}$ (L00) and $9 \mu \mathrm{m}$ (SVX) which results in an impact parameter resolution of $40 \mu \mathrm{m}$, where $30 \mu \mathrm{m}$ contribution from the beamline is included. The $z_{0}$ resolution is $70 \mu \mathrm{m}[22]$.

\section{The Central Outer Tracker}

The Central Outer Tracker is an open cell drift chamber located from $40 \mathrm{~cm}$ to $138 \mathrm{~cm}$ around the beampipe. Charged particles can ionize gas atoms an in an electric field, the produced particles can drift to the anode or cathode respectively and induce a current. The Central Outer Tracker is built of a gold covered polyester cathode plate and gold covered tungsten wires as anode in several cells. These are filled with Argon : Ethane gas in a $50: 50$ mixture with small amounts of isopropyl alcohol to obtain a good drift time. To obtain a better spatial resolution than the COT cell size the drift time, which is proportional to the distance to the hit, is measured. However, only based on drift time there is no information on which side the track has passed the sense wire. Together with the information of other cells the full track can nevertheless be reconstructed. The position resolution for a hit in the COT is $140 \mu \mathrm{m}$. The momentum resolution is

$$
\sigma_{p_{T}} / p_{T}= \begin{cases}0.15 \% \times p_{T} / \mathrm{GeV} & \text { COT only } \\ 0.07 \% \times p_{T} / \mathrm{GeV} & \mathrm{COT}+\mathrm{SVX}+\mathrm{ISL} \\ 0.05 \% \times p_{T} / \mathrm{GeV} & \text { COT beam-constrained }\end{cases}
$$

where beam-constrained assumes that the track originates in the beamline. 


\subsubsection{The Calorimeter}

The calorimeter of the CDF detector can be divided into central $(|\eta|<1.1)$, end wall and end plug calorimeter $(1.1<|\eta|<3.6)$. The naming scheme of the reconstructed objects, e.g. central or plug electrons reflects these calorimeter parts. A schematic drawing of the calorimeter can be found in Fig. 3.7. If particles travel trough calorimeter material, they interact with its atoms by several processes such as ionization, bremsstrahlung, the photoelectric effect, Compton scattering, pair production and nuclear processes. Finally electrons, photons and hadrons can lose all their energy into heat and light; muons are minimum ionizing particles and do not lose their full energy in the limited space that a calorimeter can take. A calorimeter mostly consists of several layers of absorber material, where interaction and scintillation layers alternate ${ }^{2}$. The light released by processes in the scintillation layers is collected and can be related to the energy of the incident particle. The interaction of electrons or photons and hadrons with matter is different; materials with high proton number $\mathrm{Z}$ and low mass number $\mathrm{A}$ are used to measure electromagnetic energy ${ }^{3}$, whereas materials with high mass number A are used to measure hadronic energy.

\section{The Electromagnetic Calorimeter}

The central electromagnetic calorimeter is located inside the hadronic calorimeter and consists of towers and wedges with a size of $15^{\circ}$ in azimuthal direction and 0.11 in pseudorapidity. Each wedge is built as a sampling calorimeter and made of lead absorbing and polystyrene scintillator material. The Central PreRadiator (CPR), a wire chamber located between the solenoid and the CEM, and the Central Electromagnetic Strip chamber (CES), a shower maximum detector based on a multiwire proportional counter in the middle of the CEM, help to differentiate electrons and photons using the position measurement to

\footnotetext{
${ }^{2}$ Interaction layers are present for sampling calorimeters, but not for homogeneous calorimeters.

${ }^{3}$ Electrons and photons deposit most of their energy in the electromagnetic calorimeter; hadrons deposit most of their energy in the hadronic calorimeter
} 


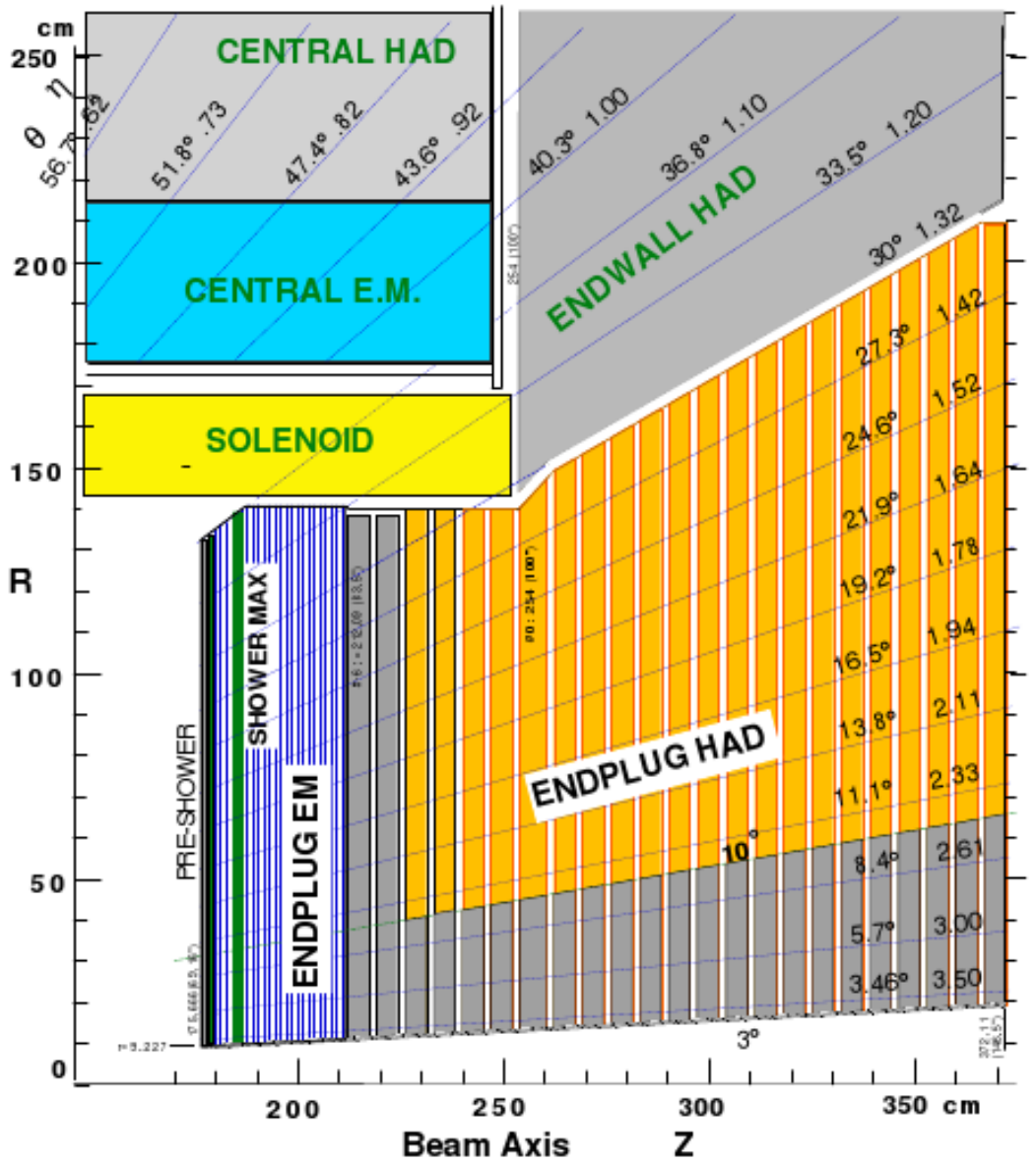

Figure 3.7: The CDF calorimeter system is consisting of the Central ElectroMagnetic calorimeter (CEM), the Central HAdronic calorimeter (CHA), the endWall HAdronic calorimeter (WHA), the endPlug ElectroMagnetic calorimeter (PEM) and the endPlug HAdronic calorimeter (PHA). 
match calorimeter deposits with tracks and to differentiate photons from $\pi^{0}$ 's based on the transverse shower profile. The energy resolution of the CEM is

$$
\sigma_{E} / E=13.5 \% \frac{1}{\sqrt{E_{T} / \mathrm{GeV}}}+2 \%
$$

\section{The Hadronic Calorimeter}

The Central HAdronic calorimeter (CHA) is a sampling calorimeter based on absorbing iron and scintillating acrylic glass material and is located outside of the CEM. The energy resolution of the $\mathrm{CHA}$ is

$$
\sigma_{E} / E=50 \% \frac{1}{\sqrt{E_{T} / \mathrm{GeV}}}+3 \%
$$

\subsubsection{The Muon Chambers}

The muon chambers are single wire tracking chambers located behind the calorimeters. Muons are minimum ionizing particles that hardly interact with the calorimeter and pass through the calorimeter material; all other particles are usually stopped in the calorimeter material. It can be assumed that every measured track outside of the calorimeter comes from a muon, however, there is background from so-called non-interacting punch through, other particles, mostly pions, that have been able to pass the calorimeter. The muon system consists of four parts: the Central MUon detector (CMU), the Central Muon uPgrade detector (CMP), the Central Muon eXtension detector (CMX) and the Intermediate MUon chambers (IMU), which are made of the Toroid Scintillator Upgrade (TSU), the Barrel MUon detector (BMU) and the Barrel Scintillator Upgrade (BSU). The $(\eta, \phi)$ coverage of these detector systems can be seen in Fig. 3.8.

\section{The Central Muon Detector}

The Central MUon detector (CMU) covers the region where $|\eta|<0.6$ and is built from $6.35 \mathrm{~cm} \times 2.68 \mathrm{~cm} \times 226 \mathrm{~cm}$ cells with a $50 \mu \mathrm{m}$ stainless steel wire in the middle. If charged particles pass through the cell, they produce ions and a current pulse can be measured in the sense wire. The CMU consists of four radial cell layers, where the first and third, 


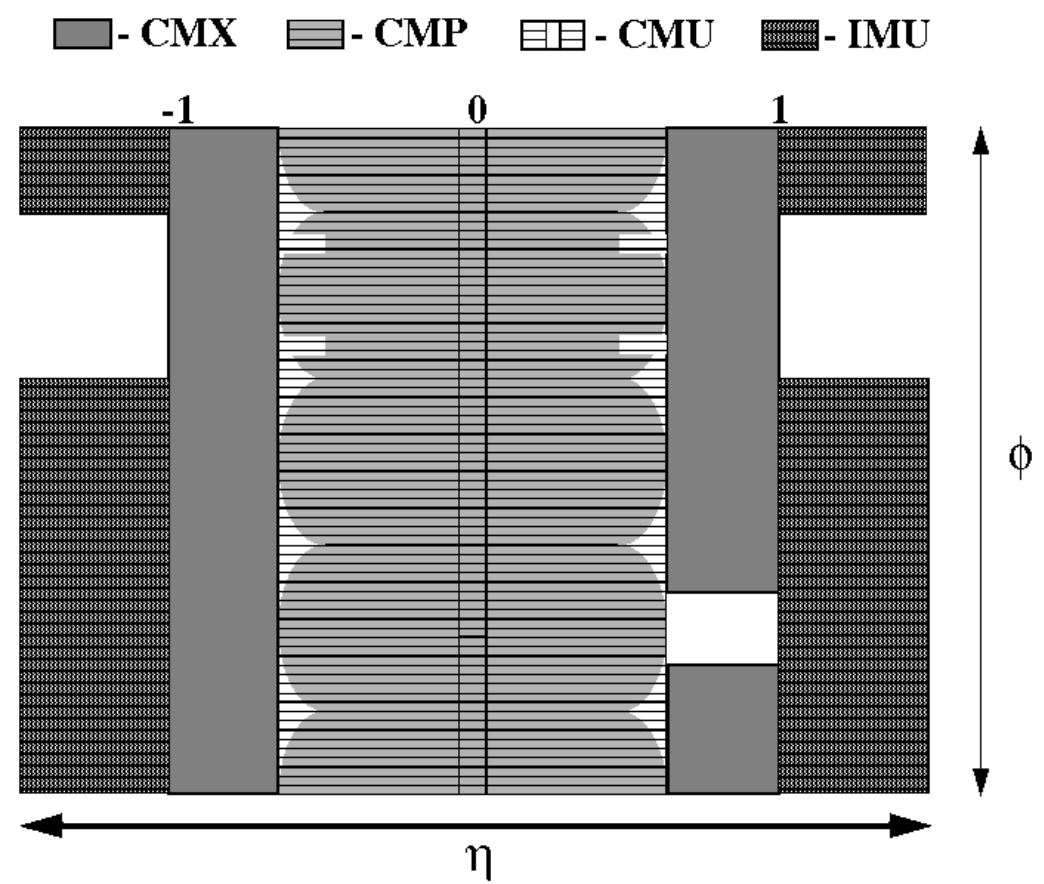

Figure 3.8: Coverage of the CDF muon detectors in the $(\eta, \phi)$-plane. CMU: Central MUon detector, CMP: Central Muon uPgrade detector, CMX: Central Muon eXtension detector, IMU: Intermediate MUon chambers consisting of the Toroid Scintillator Upgrade (TSU), the Barrel MUon detector (BMU) and the Barrel Scintillator Upgrade (BSU) 
second and fourth layers have a slight offset in $\phi$ to get a multidimensional resolution. The minimum detectable muon $p_{T}$ is $1.4 \mathrm{GeV}$.

\section{The Central Muon uPgrade Detector}

The Central Muon uPgrade detector (CMP) is located behind an extra layer of $60 \mathrm{~cm}$ steel and, similar to the $\mathrm{CMU}$, consists of a single wire drift chamber, which is operated in proportional mode and covers most of the region where $|\eta|<0.6$. Unlike the CMU, the CMP detector also includes a layer of scintillation counters with connected photomultiplier tubes, which are located on the outer side of the CMP and provide timing information for the CMP hits.

\section{The Central Muon Extension Detector}

The CMX is the outer-most muon system. It is built in a similar design to the CMP, consists of drift tubes and scintillation counter and covers the region where $0.6<|\eta|<1.0$. It can be seen in Fig. 3.8 that the CMX detector has a $30^{\circ}$ gap in the $\phi$ coverage to provide space for the Tevatron main ring and the solenoid refrigerator.

\subsubsection{The Trigger System}

At CDF events happen at a rate of several million per second even though the number of stored events has to be less 100 per second. Triggers are hardware and software components that decide which events may provide interesting physics information and are thus stored. CDF has implemented a three stage trigger system:

- Level 1 is a synchronous hardware trigger, which reduces the event input rate from 1.7 MHz to $25 \mathrm{kHz}$. It makes decisions based on fast inputs.

- Level 2 is an asynchronous mixed hard- and software trigger even though the software components are dominant. It reduces the event input rate from $25 \mathrm{kHz}$ to $550 \mathrm{~Hz}$. The level 2 trigger performs a limited event reconstruction.

- Level 3 is a software farm which reduces the event input rate from $550 \mathrm{~Hz}$ to $120 \mathrm{~Hz}$. At the stage of the level 3 trigger a full event reconstruction is done. 


\subsection{Datasets used in this Analysis}

For this analysis we use the following trigger datasets:

- high $p_{T}$ central electron (bhel*), where one central electron with $p_{T}>18 \mathrm{GeV}$ is required,

- high $p_{T}$ muon (bhmu*), where one CMUP or one CMX muon with $p_{T}>18 \mathrm{GeV}$ is required,

- SUSY dilepton (edil*), where two electrons or two muons with $p_{T}>4 \mathrm{GeV}$ for each lepton $\left(p_{T}>8,4 \mathrm{GeV}\right.$ for the last $191 \mathrm{pb}^{-1}$ of data $)$ is required.

The changed requirements for the SUSY dilepton dataset have no influence on our lepton selection due to higher thresholds in the definition of the analysis channels ${ }^{4}$. Runlist version 18 with option $(1,0,4,1)$ is used to determine good runs as we require the electromagnetic calorimeter and the muon system to be operating normally. After using the runlist there are 3636 good runs remaining, which correspond to a luminosity of $2008 \mathrm{pb}^{-1}$ for the high $p_{T}$ electron triggers. We apply correction factors

1. of 1.019 for the historical interpolation of the inelastic cross section between Run I $(\sqrt{s}=1.8 \mathrm{TeV})$ and Run II $(\sqrt{s}=1.96 \mathrm{TeV})[23]$ and

2. for a cut of $\left|z_{0}\right|<60 \mathrm{~cm}[24]^{5}$. The correction factor is 0.958 for data taking periods 0 to $7,0.968$ for periods 8 to 11 and 0.972 for periods 12 and 13 .

It is conventional to quote the integrated luminosity after the correction for the interpolation of the inelastic cross section. The integrated luminosity used by this analysis is

$$
\int \mathcal{L} d t=2046 \mathrm{pb}^{-1}
$$

for the high $p_{T}$ electron trigger. Different luminosities of the other triggers are absorbed into the trigger efficiencies. For example, if a trigger was present for only corrected $500 \mathrm{pb}^{-1}$

\footnotetext{
${ }^{4}$ See Table 4.6 for details

${ }^{5}$ For details see section 4.2.2.
} 
of $2046 \mathrm{pb}^{-1}$ and the efficiency of its path $A$ taken by lepton $i$ is $\epsilon$, we take its efficiency to be

$$
\epsilon_{i, A}^{\mathrm{corr}}=\epsilon \times \frac{500}{2046} .
$$

The uncorrected trigger efficiencies for the high $p_{T}$ electron and muon dataset and the SUSY dilepton dataset have been measured in $[25,26,27,28,29,30]$ as a function of lepton $E_{T}$. The trigger efficiency for an event is

$$
\epsilon_{A}^{\mathrm{event}}=1-\sum_{i=1}^{n} \prod_{j \neq i}\left(1-\epsilon_{j, A}\right)+(n-1) \prod_{i=1}^{n}\left(1-\epsilon_{i, A}\right),
$$

where $\mathrm{n}$ is the number of identified leptons, the second term is the efficiency for one lepton to fire the trigger and the third term compensates double-counting.

\subsection{Monte Carlo Samples used in this Analysis}

\begin{tabular}{|c|c|c|c|}
\hline Sample & Generator & $\sigma \times \mathrm{BR} / \mathrm{pb}$ & $\mathcal{L} / \mathrm{fb}^{-1}$ \\
\hline \hline $\mathrm{DY}, Z / \gamma^{*} \rightarrow e e$ & Pythia & $355 \times 1.4$ & 19.8 \\
$\mathrm{DY}, Z / \gamma^{*} \rightarrow \mu \mu$ & Pythia & $355 \times 1.4$ & 20.3 \\
$\mathrm{DY}, Z / \gamma^{*} \rightarrow \tau \tau$ & Pythia & $355 \times 1.4$ & 18.7 \\
$Z \gamma \rightarrow e e \gamma$ & Baur & $10.33 \times 1.36$ & 409 \\
$Z \gamma \rightarrow \mu \mu \gamma$ & Baur & $10.33 \times 1.36$ & 405 \\
$Z \gamma \rightarrow \tau \tau \gamma$ & Baur & $10.33 \times 1.36$ & 408 \\
$W W$ & Pythia & 1.27 & 404 \\
$W Z$ & Pythia & 0.208 & 559.6 \\
$Z Z$ & Pythia & 2.116 & 491.8 \\
$t \bar{t}$ & Pythia & 6.9 & 593.0 \\
\hline
\end{tabular}

Table 3.1: Monte Carlo background samples. Diboson samples include the decay of off-shell particles.

Monte Carlo samples for the background estimation were produced with the generators in Table 3.1. For signal Monte Carlo we use the CDF MCPRoduction 6.1.4mc standard MC tarballs with PYTHIA 6.409[31] $(6.216)^{6}$, parton distribution function CTEQ5L[32], spectrum calculated by ISASUgra from ISAJET 7.75[33] $(7.51)^{6}$ and $\tau$ decays by TAUOLA[34]. Cross

\footnotetext{
${ }^{6}$ For a detailed discussion of the used versions of IsAJET and PYTHIA see appendix B.
} 
sections for supersymmetric processes are calculated at next to leading order with PROSPINO $2.0[35]$.

In the analysis section of this thesis the signal predictions are obtained from a Monte Carlo sample produced with mSUGRA parameters as defined as benchmark point BP1 in section 2.2.4 with the parameters

- $m_{0}=60 \mathrm{GeV} / \mathrm{c}^{2}$

- $m_{1 / 2}=190 \mathrm{GeV} / \mathrm{c}^{2}$

- $\tan \beta=3$

- $A_{0}=0 \mathrm{GeV}$

- $\mu>0$. 


\section{Chapter 4}

\section{The Trilepton Analysis}

The basis of this thesis is a search for supersymmetric chargino-neutralino production in the three leptons and missing transverse energy final state. Setup as an unbiased counting experiment, several channels based on lepton flavor are defined and the number of events observed in data of the CDF II detector is compared with the expectation for the Standard Model background.

\subsection{The Trilepton Signature}

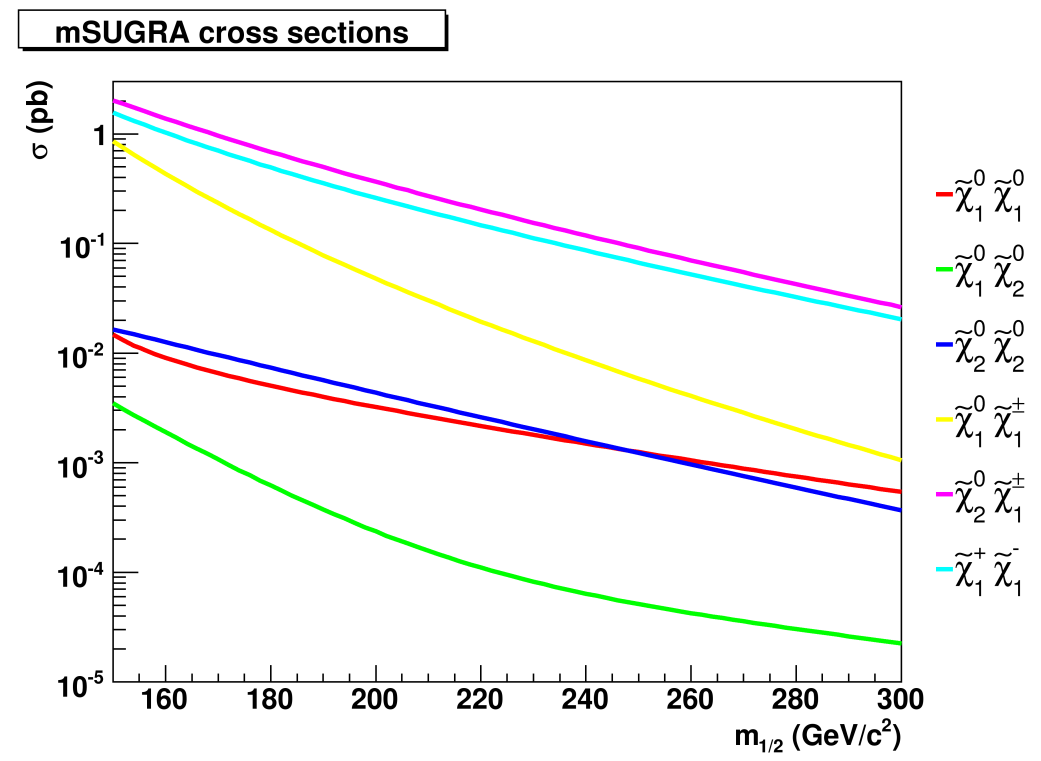

Figure 4.1: Cross sections at next-to-leading order precision for the production of different chargino-chargino, chargino-neutralino and neutralino-neutralino pairs in $p \bar{p} \sqrt{s}=1.96 \mathrm{TeV}$ collisions at the Tevatron. The cross sections for the production of pairs of the form $\tilde{\chi}_{i}^{0} \tilde{\chi}_{j}^{ \pm}$ are the sum for the cross sections for positively and negatively charged chargino production. The mass spectrum was calculated with ISAJET 7.75 in mSUGRA as a function of $m_{1 / 2}$ at benchmark point BP1; the cross section was calculated with PROSPINO 2.0. 
At the Tevatron associate production of chargino $\tilde{\chi}_{1}^{ \pm}$and neutralino $\tilde{\chi}_{2}^{0}$ can happen in $p \bar{p}$ collisions. The s-channel production occurs via exchange of an off-shell $W^{ \pm *}$ boson; the t-channel production via exchange of an off-shell squark $\tilde{q}^{*}$. The s- and t-channel interfere destructively; for high squark masses the t-channel production is suppressed and the cross section increases[36]. Feynman diagrams for the production channels can be found in Fig. 4.2. The production cross section for $\tilde{\chi}_{1}^{ \pm} \tilde{\chi}_{2}^{0}$ at benchmark point $\mathrm{BP} 1^{1}$ at the precision of a next to leading order calculation with PROSPINO $2.0[35]^{2}$ is

$$
\sigma=(0.500 \pm 0.050) \mathrm{pb}
$$

so that for an integrated luminosity $\int \mathcal{L} d t=2.0 \mathrm{fb}^{-1}$ a total of $1000 \tilde{\chi}_{1}^{ \pm} \tilde{\chi}_{2}^{0}$ events are expected for direct production. This search is focusing on the leptonic decay of chargino
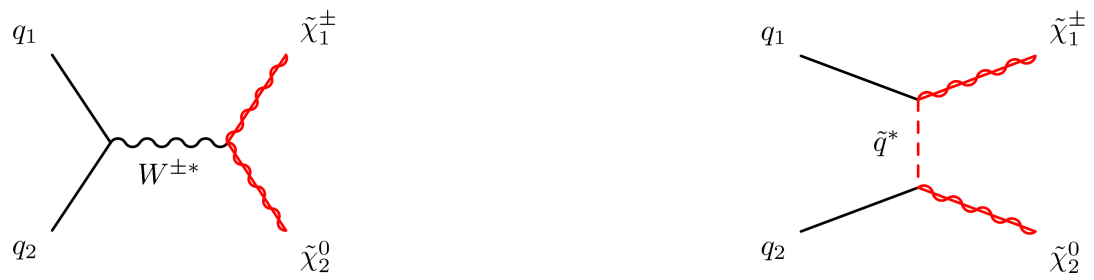

Figure 4.2: Leading order Feynman diagrams for the associated production of chargino and neutralino in $p \bar{p}$ collisions

and neutralino. Figure 4.3 shows the dominant leptonic decays of chargino and neutralino

$$
\begin{aligned}
& \tilde{\chi}_{1}^{ \pm} \rightarrow \tilde{\chi}_{1}^{0} W^{ \pm *} \rightarrow \tilde{\chi}_{1}^{0} l^{ \pm} \nu_{l} \\
& \tilde{\chi}_{1}^{ \pm} \rightarrow \tilde{\tau}_{1}^{ \pm} \nu_{\tau} \rightarrow \tilde{\chi}_{1}^{0} \tau^{ \pm} \nu_{\tau} \\
& \tilde{\chi}_{1}^{ \pm} \rightarrow \tilde{\nu}_{l} l^{ \pm} \rightarrow \tilde{\chi}_{1}^{0} l^{ \pm} \nu_{l} \\
& \tilde{\chi}_{2}^{0} \rightarrow \tilde{\chi}_{1}^{0} Z^{0 *} \rightarrow \tilde{\chi}_{1}^{0} l^{+} l^{-} \\
& \tilde{\chi}_{2}^{0} \rightarrow \tilde{l}_{R}^{ \pm} l^{\mp} \rightarrow \tilde{\chi}_{1}^{0} l^{+} l^{-},
\end{aligned}
$$

where $l=e, \mu, \tau$ and $\tilde{l}_{R}^{ \pm}=\tilde{e}_{R}^{ \pm}, \tilde{\mu}_{R}^{ \pm}, \tilde{\tau}_{1}^{ \pm}$. The decay of the chargino into right-handed selectrons and smuons is highly suppressed. The chargino is a mixed mass eigenstate with a charged Wino and a Higgsino component; Fig. 2.2 shows the couplings of the Higgsino and charged

${ }^{1}$ mSUGRA $m_{0}=60 \mathrm{GeV} / \mathrm{c}^{2}, m_{1 / 2}=190 \mathrm{GeV} / \mathrm{c}^{2}, \tan \beta=3, A_{0}=0 \mathrm{GeV}, \mu>0$

${ }^{2}$ The error calculated by PROSPINO is too low; a $10 \%$ error is assumed[37]. 
Wino component of the chargino. The charged Wino component couples to left-handed sleptons only. As there is very low mixing of the left- and right-handed selectrons and smuons the coupling of the chargino's Wino component to the lighter selectron and smuon is negligible. The lighter stau $\tilde{\tau}_{1}$ is a mixture of the left- and right-handed stau and the charged Wino can couple to the left-handed component of the $\tilde{\tau}_{1}$. The Higgsino component of the chargino couples via a Yukawa term to sleptons. In the approximation of Eqn. 2.20 the coupling of the Higgsino to selectron and smuon is negligible, whereas there is a nonnegligible coupling to the stau. A more detailed analysis of the chargino and neutralino branching ratios as a function of the mSUGRA parameters can be found in section 5.1.

As the lightest supersymmetric particles $\tilde{\chi}_{1}^{0}$ and the neutrino $\nu_{l}$ escape the detector undetected and result in missing transverse energy, the signature for the leptonic decay of chargino and neutralino in Eqns. 4.2 to 4.6 is three leptons and missing transverse energy, the so-called trilepton signature. One lepton comes from the decay of the chargino; two leptons from the decay of the neutralino. The production of chargino and neutralino in the trilepton channel is one of the most promising searches for supersymmetry at the Tevatron and is often referred to as the "golden" channel. [38] is examining several final states of supersymmetric interaction in a constrained MSSM model for their discovery at the Tevatron.

\subsection{Event Reconstruction}

Electrons and muons can be identified directly based on their signals in the tracker, the calorimeter and the muon system, however $\tau$ leptons decay in the detector and can only be identified via their decay products. A list of the decay channels of the $\tau$ lepton and their branching ratios can be found in Table 4.1. If the $\tau$ lepton decays into an electron or a muon, it is possible to identify them in the respective categories provided that they pass the quality criteria. In approximately $50 \%$ of all cases the $\tau$ lepton decays into a hadronic one-prong final state. In order to get sensitivity to this hadronic one-prong decay of the $\tau$ lepton the analysis is identifying isolated tracks. The isolation criterion is needed to suppress background; three-prong decays usually do not pass the isolation criterion. 

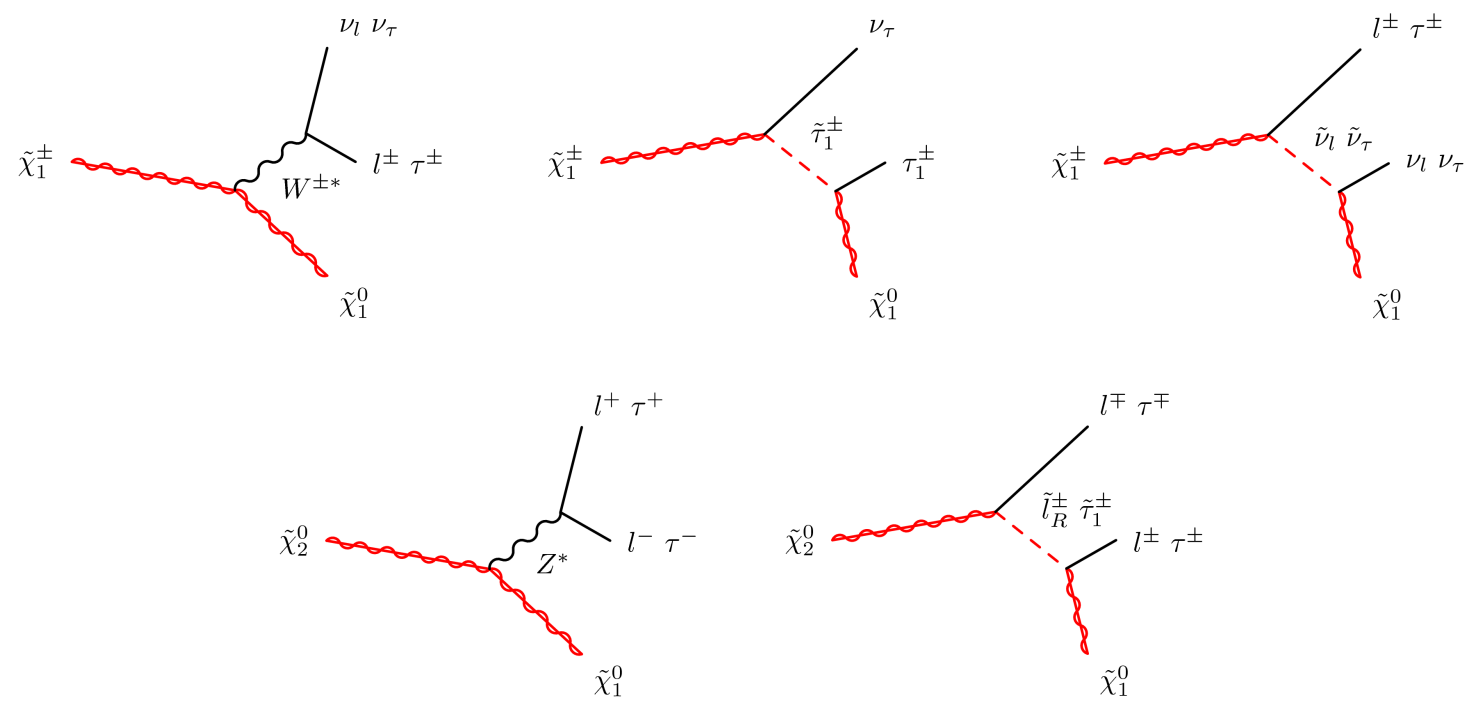

Figure 4.3: Dominant decay channels of the neutralino $\tilde{\chi}_{2}^{0}$ and the chargino $\tilde{\chi}_{1}^{ \pm}$into leptons. It is $l=e, \mu$.

\begin{tabular}{|c|l|}
\hline$\tau^{-} \rightarrow$ & $\mathrm{BR}$ \\
\hline \hline one-prong decay & $85.4 \%$ \\
\hline$\mu^{-} \bar{\nu}_{\mu} \nu_{\tau}$ & $17.4 \%$ \\
\hline$e^{-} \bar{\nu}_{e} \nu_{\tau}(+\gamma)$ & $17.9 \%$ \\
\hline$\pi^{-} \nu_{\tau}$ & $10.9 \%$ \\
\hline$\pi^{-} \pi^{0} \nu_{\tau}$ & $25.5 \%$ \\
\hline$\pi^{-} \pi^{0} \pi^{0} \nu_{\tau}$ & $9.3 \%$ \\
\hline$\pi^{-} \pi^{0} \pi^{0} \pi^{0} \nu_{\tau}$ & $1.0 \%$ \\
\hline$X^{-} \nu_{\tau}$ & $1.2 \%$ \\
\hline$K(892)^{-} \nu_{\tau}$ & $1.2 \%$ \\
\hline
\end{tabular}

\begin{tabular}{|c|l|}
\hline$\tau^{-} \rightarrow$ & $\mathrm{BR}$ \\
\hline \hline three-prong decay & $14.6 \%$ \\
\hline$\pi^{-} \pi^{+} \pi^{-} \nu_{\tau}$ & $9.3 \%$ \\
\hline$\pi^{-} \pi^{+} \pi^{-} \pi^{0} \nu_{\tau}$ & $4.6 \%$ \\
\hline five-prong decay & $0.1 \%$ \\
\hline
\end{tabular}

Table 4.1: Branching ratios for the different decay channels of the $\tau^{-}$lepton according to [1]. Decay channels with braching ratio smaller than $1 \%$ are neglected. 


\subsubsection{Event Vertex}

The event or primary vertex is defined as the place where the $p \bar{p}$ collision happens and where most particles originate. The vertex finder algorithm ZVERTEXFINDER generates a seed list of track vertex candidates with certain quality requirements based on silicon and COT hits. $z_{0}$ is the distance from the center of the detector to the intersection of the beamline and the interpolation of the track. Vertices with a $z_{0}$ distance smaller than $3 \mathrm{~cm}$ are joined and a new weighted $z_{0}$ is calculated for the vertex compound. If there are no vertices or vertex compounds with a distance less than $3 \mathrm{~cm}$ left, the vertex or vertex compound with the highest transverse energy and CDF quality 12 is chosen as the event vertex $z_{V}$. For events, which we select, we require

$$
\left|z_{V}\right|<60 \mathrm{~cm}
$$

\subsubsection{Tracks}

\begin{tabular}{|l|l|}
\hline ID Cut & Track \\
\hline \hline Track $\left|z_{0}\right|$ & $\leq 60 \mathrm{~cm}$ \\
Track $\left|z_{0}-z_{V}\right|$ & $\leq 5 \mathrm{~cm}$ \\
$p_{T}$ & $\geq 5 \mathrm{GeV}$ \\
NAxialSeg( $\geq 5$ Hits $)$ & $\geq 3$ \\
NStereoSeg( $\geq 5$ Hits $)$ & $\geq 3$ \\
Fractional Track Isolation & 0 \\
\hline
\end{tabular}

Table 4.2: Identification requirements for isolated tracks. In addition we require that the track is not associated to a selected electron or muon.

In this analysis tracks with hits in the Central Outer Tracker are used; if available, hits in the silicon system are matched. The track reconstruction algorithm[39, 40] looks for seeds, which are hits in three neighboring layers, and fits a straight line. Close hits are added, the line fit is adapted and a segment-linking algorithm forms a track from the seeds. Tracks are also fit by the histogram-tracking algorithm. A histogram is filled with the likelihood, that 
a track might pass through a hit as a function of the track radius. If a bin content reaches a certain threshold, a track is fit.

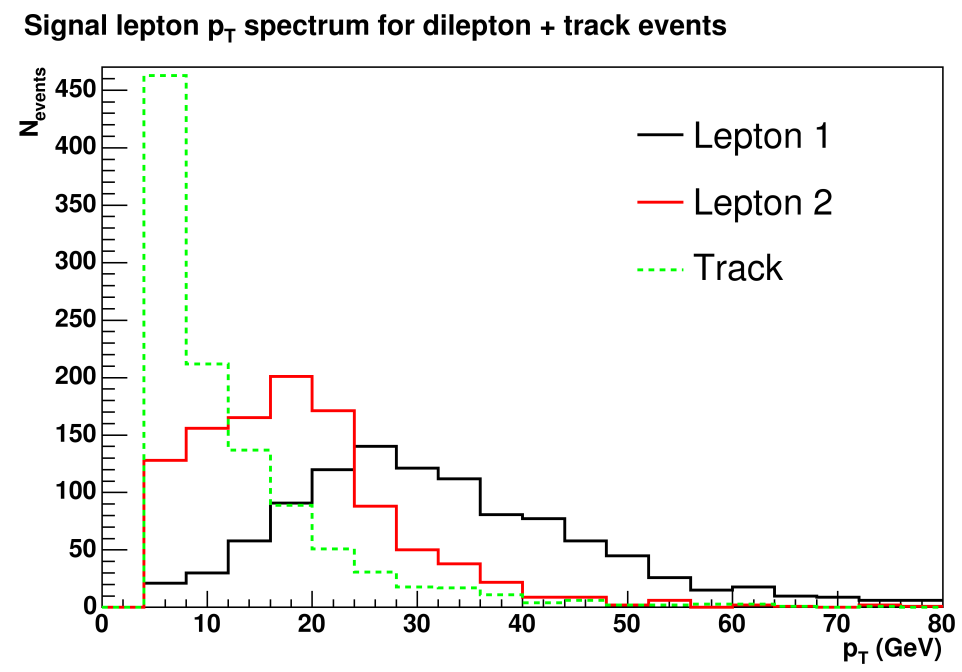

Figure 4.4: Distribution of transverse momentum for electrons, muons and isolated tracks in dilepton + track events at generator level for benchmark point BP1. The leptons are ordered by decreasing $p_{T}$. A cut of $p_{T}>4 \mathrm{GeV}$ was applied to remove leptons from meson decays.

For the identification of a track we require the conditions listed in Table 4.2, where

Track $\left|z_{0}\right|$ is the distance from the center of the detector to the intersection of the beamline and the interpolation of the track,

Track $\left|z_{0}-z_{V}\right|$ is the distance from $z_{0}$ to the event vertex $z_{V}$.

$p_{T}$ is the transverse momentum of the track,

NAxialSeg( $\geq 5$ Hits) and NStereoSeg( $\geq 5$ Hits) are the numbers of axial and stereo segments in the central outer tracker with at least 5 hits,

Fractional Track Isolation is defined as the ratio of the scalar sum of $p_{T}$ of all tracks in a cone of 0.4 in the $(\eta, \phi)$-plane with $p_{T}>0.4 \mathrm{GeV}$ and $p_{T}$ of the candidate track. The tracks considered need to satisfy

$$
\left|z_{0}^{\text {surrounding }}-z_{0}^{\text {candidate }}\right|<4 \mathrm{~cm} .
$$

We require a fractional track isolation of 0 which is equivalent to having no tracks passing these requirements in the cone. 
By restricting the choice of tracks to tracks from the COT an additional implicit pseudorapidity cut $|\eta|<1.5$ is introduced. The transverse momentum distribution for tracks in signal Monte Carlo can be found in Fig. 4.4.

\subsubsection{Electrons}

For the reconstruction of electrons (and muons) we follow the standard CDF joint physics definition[41] for most requirements. In the CDF detector a central electron is a COT track that is matched to a deposit in the electromagnetic calorimeter. We identify central tight (TCE) and central loose electrons (LCE), where central refers to $|\eta|<1.1$ and tight and loose refers to the degree of the identification requirements. Electrons in the forward region of the detector, the so-called Phoenix or plug electrons, are not reconstructed for this analysis. We require the conditions documented in Table 4.3, where

CEM fiduciality is the requirement for the calorimeter deposit to be away from problematic regions of the central electromagnetic calorimeter. The fiducial volume doesn't include tower 9 and the chimney in tower 7, where the cables exit the detector. Additionally a signal in a CES wire and strip cluster is required.

$E_{T}=E \sin \theta$ and $p_{T}=p \sin \theta$ are the transverse energy measured in the calorimeter and the transverse momentum measured in the tracker.

Had/EM is the ratio of hadronic and electromagnetic energy. It is expected that an electron deposits most of its energy in the electromagnetic calorimeter.

CES $\chi_{\text {strip }}^{2}$ is a statistic measure for how similar the CES shower profile is to test beam data.

Isolation energy is the energy in a cone of $\Delta R=\sqrt{\Delta \eta^{2}+\Delta \phi^{2}}=0.4$ around the calorimeter deposit.

Fractional isolation is the ratio of the isolation energy and the energy of the cluster itself.

$\mathbf{E} / \mathbf{p}$ is the ratio of energy deposit and momentum of the track, which is, due to the small electron mass, usually close to 1. 


\begin{tabular}{|l|l|l|}
\hline ID Cut & Central Tight Electron & Central Loose Electron \\
\hline \hline Fiduciality & $\mathrm{CEM}$ & $\mathrm{CEM}$ \\
Track $\left|z_{0}\right|$ & $<60 \mathrm{~cm}$ & $<60 \mathrm{~cm}$ \\
Track $\left|z_{0}-z_{V}\right|$ & $<5 \mathrm{~cm}$ & $<5 \mathrm{~cm}$ \\
$E_{T}$ & $\geq 4 \mathrm{GeV}$ & $\geq 4 \mathrm{GeV}$ \\
$p_{T}$ & $\geq 4 \mathrm{GeV}$ & $\geq 4 \mathrm{GeV}$ \\
Had/EM Energy & $<0.055+0.00045 \quad \times$ & $<0.055+0.00045 \quad \times$ \\
& $E_{E M} / \mathrm{GeV}$ & $E_{E M} / \mathrm{GeV}$ \\
NAxialSeg( $\geq 5 \mathrm{Hits})$ & $\geq 3$ & $\geq 3$ \\
NStereoSeg $(\geq 5 \mathrm{Hits})$ & $\geq 2$ & $\geq 2$ \\
CES $\chi_{\text {strip }}^{2}$ & $<10$ & $<20$ \\
Fractional Isolation & $<0.1$ if $E_{E M, T}>20 \mathrm{GeV}$ & $<0.1$ \\
Isolation Energy & $<2 \mathrm{GeV}$ if $E_{E M, T}>$ & - \\
& $20 \mathrm{GeV}$ & \\
E/p & $<2$ if Track $p_{T}<50 \mathrm{GeV}$ & - \\
Lshr & $<0.2$ & \\
charge $\times \Delta X$ & $>-3 \mathrm{~cm}$ and $<1.5 \mathrm{~cm}$ & - \\
$|\Delta Z|$ & $<3 \mathrm{~cm}$ & - \\
\hline
\end{tabular}

Table 4.3: Electron identification requirements for tight central and loose central electrons 
Lshr is defined as[42]

$$
\operatorname{Lshr}=0.14 \times \frac{\sum_{i}\left(M_{i}-P_{i}\right)}{\sqrt{\left(0.14 \sqrt{E_{E M}}\right)^{2}+\sum_{i} \Delta P_{i}^{2}}},
$$

where all numbers are in $\mathrm{GeV}, i$ sums over the calorimeter towers adjacent to the seed tower, $M_{i}$ is the measured energy deposit in tower $i, P_{i}$ is the predicted energy deposit in tower $i$ based on test beam data, $\Delta P_{i}$ is a measure of the error of $P_{i}$ and $E_{E M}$ is the total electromagnetic energy in the considered cluster.

Charge $\times \Delta X$ is the charge of the track multiplied by the distance in the $(r, \phi)$-plane between the interpolation of the COT track and the nearest CES cluster.

$|\Delta Z|$ is the distance in the $(r, z)$-plane between the interpolation of the COT track and the nearest CES cluster.

The assignment to the categories is exclusive; a candidate lepton that is able to pass the requirements for TCE and LCE is categorized as a TCE electron.

It can be seen in Fig. 4.5, that for a good signal acceptance, it is crucial to identify leptons with low transverse momentum or energy. For increasing $\tan \beta$ this becomes even more important as the number of $\tau$ leptons increases ${ }^{3}$ and electrons and muons, which are decay products of a $\tau$ leptons, are usually softer than electrons or muons, that originate from neutralinos, sleptons or off-shell $W^{ \pm *}$ or $Z^{0 *}$ bosons. For this reason the requirements on the lepton transverse momentum and energy are significantly lower than the CDF joint physics definition.

\subsubsection{Muons}

For the identification of muons information from the tracker, the calorimeter and the muon system (for CMUP and CMX muons) is used. The energy deposited in the calorimeter has to be consistent with the signal from a minimum ionizing particle. The CMUP, CMX category is named after the muon systems, where the muon has left a stub; CMIO muons are stubless. For the reconstruction of CMUP or CMX muons a stub in the muon system

\footnotetext{
${ }^{3}$ See section 5.1 .3 for details.
} 


\begin{tabular}{|c|c|c|}
\hline ID Cut & CMUP, CMX & CMIO \\
\hline Fiduciality & $(\mathrm{CMU}$ and $\mathrm{CMP})$ or $\mathrm{CMX}$ & $\begin{array}{l}\text { not (CMU and CMP), not } \\
\text { CMX }\end{array}$ \\
\hline$|\eta|$ & $\leq 1.0$ & $\leq 1.0$ \\
\hline $\mathrm{BC} p_{T}$ & $\geq 4 \mathrm{GeV}$ & $\geq 10 \mathrm{GeV}$ \\
\hline Track $\left|z_{0}\right|$ & $\leq 60 \mathrm{~cm}$ & $\leq 60 \mathrm{~cm}$ \\
\hline Track $\left|z_{0}-z_{V}\right|$ & $\leq 5 \mathrm{~cm}$ & $\leq 5 \mathrm{~cm}$ \\
\hline$E_{T}$ & $\geq 5 \mathrm{GeV}$ & $\geq 5 \mathrm{GeV}$ \\
\hline $\begin{array}{l}\text { Stub Matching } \Delta X \\
(\mathrm{CMU}, \mathrm{CMP}, \mathrm{CMX})\end{array}$ & $\leq 7,5,6$ & - \\
\hline Track $\chi^{2}$ (Data) & $<2.3$ & $<2.3$ \\
\hline $\begin{array}{l}\text { Corrected } d_{0} \text { (for } \mathrm{Si} \\
\text { Hits, for no Si Hits) }\end{array}$ & $\leq 0.2,0.02$ & $\leq 0.2,0.02$ \\
\hline NAxialSeg( $\geq 5$ Hits $)$ & $\geq 3$ & $\geq 3$ \\
\hline NStereoSeg( $\geq 5$ Hits $)$ & $\geq 2$ & $\geq 3$ \\
\hline Had Energy & $\leq 6 \mathrm{GeV}+$ sliding & $\leq 6 \mathrm{GeV}+$ sliding \\
\hline EM Energy & $\leq 2 \mathrm{GeV}+$ sliding & $\leq 2 \mathrm{GeV}+$ sliding \\
\hline EM + Had Energy & $\geq 0.1 \mathrm{GeV}$ & $\geq 0.1 \mathrm{GeV}$ \\
\hline Fractional Isolation & $\begin{array}{l}\leq 0.1 \text { for track } p_{T}> \\
20 \mathrm{GeV}\end{array}$ & $\leq 0.1$ \\
\hline Isolation Energy & $\begin{array}{l}\leq 2 \mathrm{GeV} \text { for track } p_{T}< \\
20 \mathrm{GeV}\end{array}$ & \\
\hline
\end{tabular}

Table 4.4: Muon identification requirements for CMUP/CMX and CMIO muons. "+ sliding" stands for the requirements Had Energy $\leq 6 \mathrm{GeV}+$ $\max (0 \mathrm{GeV}, 0.0028 \times(p-100 \mathrm{GeV}))$ if track $p_{T}>20 \mathrm{GeV}$, Had Energy $<3.5+1 / 8 \times \operatorname{track} p_{T}$ if track $p_{T} \leq 20 \mathrm{GeV}$ and EM Energy $\leq 2 \mathrm{GeV}+$ $\max (0 \mathrm{GeV}, 0.0115 \times(p-100 \mathrm{GeV}))$. 


\section{Signal lepton $\mathrm{p}_{\mathrm{T}}$ spectrum for trilepton events}

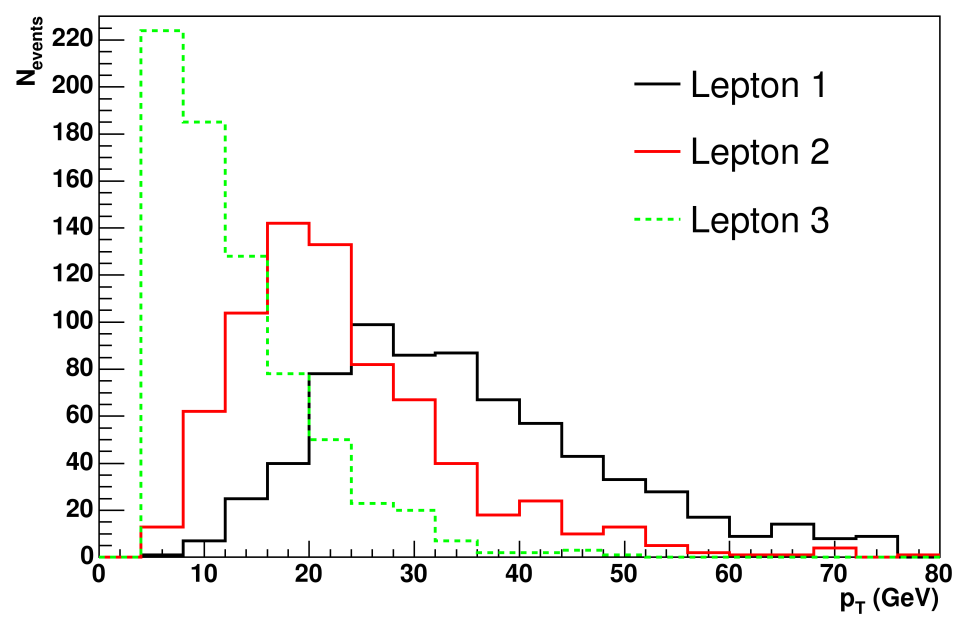

Figure 4.5: Distribution of transverse momentum for electrons and muons in trilepton events at generator level for benchmark point BP1. The leptons are ordered by decreasing $p_{T}$. A cut at $p_{T}=4 \mathrm{GeV}$ was applied to remove leptons from meson decays.

is used as a seed and the best matching calorimeter deposits and tracks are identified. In addition we pose the requirements listed in Table 4.4, where

$\eta$ is the pseudorapidity of the muon given by

$$
\eta=-\ln \tan \frac{\theta}{2}
$$

Fiduciality here refers to the appropriate regions in the CMU, CMP or CMX muon system,

BC $p_{T}$ is the beam constrained track $p_{T}$,

Stub Matching $\Delta X$ is the distance in the $(r, \phi)$-plane between the interpolation of the COT track and the muon stub,

Track $\chi^{2}$ (Data) is a measurement for the agreement of the track and the hits in the COT and reduces the background from poorly reconstructed tracks, primarily from kaons that decay in flight,

Corrected $d_{0}$ is the impact parameter of the track, the distance between the beamline and the position of the track's interpolation in the $(r, \phi)$-plane.

All other requirements have been explained in section 4.2.3. Similar to the assignment of electron categories the assignment of muon categories is exclusive; a candidate lepton that is 
able to pass the requirements for CMUP/CMX and CMIO is categorized as a CMUP/CMX muon.

\subsubsection{Jets}

Jets are clusters of towers with signals in the hadronic and electromagnetic calorimeters. In this analysis jets were identified with the JETCLU algorithm and a cone radius of $\Delta R=$ $0.4[43]$. The algorithm is described below:

1. A list of seed towers with $E_{T}=E_{\text {em }} \sin \theta_{\text {em }}+E_{\text {had }} \sin \theta_{\text {had }}>1.0 \mathrm{GeV}$ is created.

2. The highest $E_{T}$ seed tower (and subsequently all other to that point unused seed towers) are defined as a precluster and seed towers within a cone of

$$
\Delta R=\sqrt{\left(\eta_{\text {tower }}-\eta_{\text {precluster }}\right)^{2}+\left(\phi_{\text {tower }}-\phi_{\text {precluster }}\right)^{2}}=0.4
$$

around the precluster are exclusively assigned to the precluster. It has to be noted that $\eta$ and $\phi$ of the precluster change when towers are added.

3. Other calorimeter towers within the cone and $E_{T}>0.1 \mathrm{GeV}$ are added.

4. The overlap fraction of preclusters is calculated as the energy of common towers divided by the energy of the smaller cluster. If the overlap fraction is greater than $75 \%$ the clusters are merged; otherwise common towers are assigned exclusively to the nearer cluster.

5. Final clusters are regarded as jets.

After clustering the jet energy is corrected to level 5 which includes online/offline calibrations (for example corrections for minimum ionizing particles, level 0), $\eta$ dependent corrections (level 1), correction for multiple interactions as a function of the number of vertices in the event (level 4) and corrections for non-linearity and energy loss in the uninstrumented regions of the calorimeter (level 5). A systematic error for the jet energy scale is applied according to section 4.6.2. In this analysis we select jets with raw $E_{T}>8 \mathrm{GeV}$, level-5 corrected $E_{T}>15 \mathrm{GeV}$ and EM fraction $<0.9$. Jets are not used as a direct analysis object, but to correct missing transverse energy and to reject events with high hadronic activity or jets that are close to analysis objects. 


\subsubsection{Identification Scale Factors}

If the identification efficiency for leptons is compared in data and in Monte Carlo, it turns out that there are differences. To account for these differences a scale factor for the identification of leptons is introduced. A description of how these scale factors are obtained can be found in [44]. The track identification scale factors are assumed to be 1.

\begin{tabular}{|l|l|l|}
\hline Lepton & $E_{T}$ range & Scale factor \\
\hline \hline TCE & $\geq 20 \mathrm{GeV}$ & $0.979 \pm 0.006$ \\
& $8 \ldots 20 \mathrm{GeV}$ & $0.96 \pm 0.02$ \\
& $5 \ldots 8 \mathrm{GeV}$ & $0.88 \pm 0.16$ \\
$\mathrm{LCE}$ & $\geq 20 \mathrm{GeV}$ & $0.964 \times 1.025 \pm 0.03$ \\
& $8 \ldots 20 \mathrm{GeV}$ & $0.968 \times 1.015 \pm 0.03$ \\
& $5 \ldots 8 \mathrm{GeV}$ & $0.97 \pm 0.10$ \\
CMUP & $\geq 20 \mathrm{GeV}$ & $0.92 \pm 0.006$ \\
& $8 \ldots 20 \mathrm{GeV}$ & $0.90 \pm 0.04$ \\
& $5 \ldots 8 \mathrm{GeV}$ & $0.87 \pm 0.04$ \\
CMX & $\geq 20 \mathrm{GeV}$ & $0.97 \pm 0.01$ \\
& $8 \ldots 20 \mathrm{GeV}$ & $0.91 \pm 0.04$ \\
& $5 \ldots 8 \mathrm{GeV}$ & $0.88 \pm 0.04$ \\
CMIO & $\geq 20 \mathrm{GeV}$ & $1.0 \pm 0.01$ \\
& $10 \ldots 20 \mathrm{GeV}$ & $1.01 \pm 0.057$ \\
\hline
\end{tabular}

Table 4.5: Lepton identification scale factors

\subsubsection{Missing Transverse Energy}

Neutrinos and the lightest neutralino interact with the detector material only via the weak force, so that their energy can not be measured. However they carry away energy and momentum leaving an unbalance. As the Tevatron is a hadron collider the longitudinal energy of the interacting quarks can not be unambiguously determined. It is therefore 
necessary to restrict the search for a momentum unbalance to the transverse component. The transverse energy of an event is defined as

$$
\vec{E}_{T}^{\mathrm{raw}}=\sum_{i=1}^{N_{\text {towers }}} E_{\text {tower }, i} \sin \theta_{i} \vec{n}_{i},
$$

where $N_{\text {towers }}$ is the number of towers in the calorimeter, $E_{\text {tower, } i}$ is the energy deposited in calorimeter tower $i, \vec{n}_{i}$ is a unit vector pointing pointing from the center of the detector to the center of calorimeter tower $i$ and $\theta_{i}$ is the angle between $\vec{n}_{i}$ and the beam axis, so that $\sin \theta_{i} \vec{n}_{i}$ is the transverse component of $\vec{n}_{i}$. The z component of $\vec{E}_{T}^{\mathrm{raw}}$ is set to 0 . Missing transverse energy $\vec{E}_{T}$ can thus be defined by

$$
\vec{E}_{T}^{\text {raw }}=-\vec{E}_{T}^{r a w}
$$

but it is often used as a scalar quantity. Missing $E_{T}$ is vulnerable towards mismeasurements. Corrections for overlapping jets and tracks, muons, jets and tracks that are not associated to a muon, but have a signal in the muon chambers, are applied to account for mismeasurements.

- If an isolated track that is not coming from a minimum ionizing muon and a jet have a distance $\Delta R=\sqrt{\Delta \eta^{2}+\Delta \phi^{2}}<0.4$, the missing $E_{T}$ is corrected for the energy difference of the jet and the track. If the track $E_{T}$ is lower than the jet $E_{T}$, the track is probably included in the jet; if the jet $E_{T}$ is lower than the track $E_{T}$, the jet $E_{T}$ is certainly measured too low by mistake.

- For jets with $|\eta|>2.5$ and $E_{T}>15 \mathrm{GeV}$ the missing $E_{T}$ is corrected for the difference in raw $E_{T}$ and level-5 corrected $E_{T}{ }^{4}$.

- For selected muons the missing $E_{T}$ is corrected for the difference between muon track $p_{T}$ and $E_{T}=\frac{E_{e m}+E_{h a d}}{\cosh \eta}$ as muons are minimum ionizing particles.

- In the dilepton + track channels ${ }^{5}$ missing $E_{T}$ is corrected for the difference between $E_{T}$ and track $p_{T}$ of of a track if there is no signal in the muon chamber and $E / p<1.0$.

The effects of the corrections on the missing $E_{T}$ distribution can be seen in Fig. 4.6.

\footnotetext{
${ }^{4} \eta$ is calculated assuming $z_{0}=0$.

${ }^{5}$ For details on the definition of analysis channels see Table 4.6.
} 


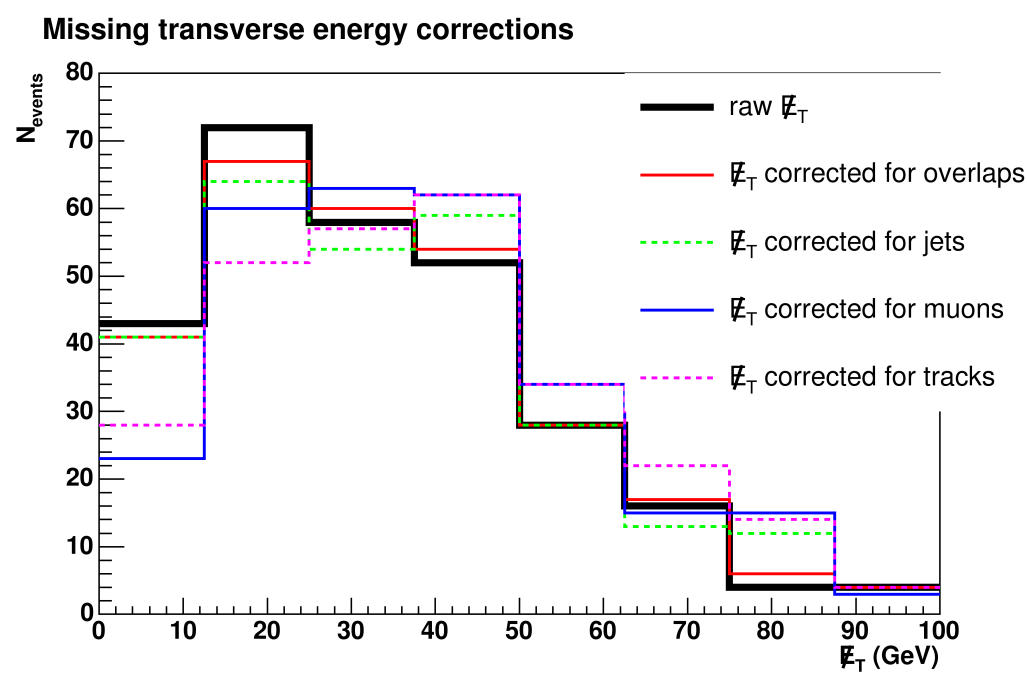

Figure 4.6: Effects of successively applied corrections to missing $E_{T}$ for benchmark point $\mathrm{BP} 1$ in the $l_{t} l_{t} T$ channel at the level of full detector simulation. The histograms for the corrections contain all previous corrections, e.g. the value for $\mathbb{E}_{T}$ corrected for tracks contains all other corrections.

\subsubsection{Event Vetoes}

We require an event vertex to be selected according to section 4.2.1. Events where no event vertex can be selected are rejected.

\section{Cosmic Ray Veto}

Cosmic rays are energetic particles from space that produce pions and kaons when they interact with gas atoms in the earth's atmosphere. Pions and kaons are unstable and can decay into muons. As muons are minimum ionizing particles their interaction with the earth's atmosphere is weak enough, so that the energetic ones are able to reach the earth's surface and can be seen in the CDF detector. When a cosmic muon passes the CDF detector it can be reconstructed as two oppositely charged muons. When going into the detector the muon is travelling in opposite direction to a muon that would come from the interaction point and the muon charge is misidentified.

In this analysis we use the CDF Run II Cosmic Ray Tagger[45] to reject events with cosmic muons. The Cosmic Ray Tagger is rejecting events based on their timing and geometry information as muons from cosmic rays are expected to be out-of-time and backto-back. 


\section{Photon Conversion Veto}

The process of a photon decaying into two oppositely charged electrons is called a conversion.

In this analysis a pair of electrons having opposite charge may be rejected if $\Delta \cot \theta$ and the minimal separation of the associated tracks are consistent with a conversion. The photon conversion removal efficiency is different for data and Monte Carlo; a scale factor is applied to account for different conversion removal efficiencies[46].

\section{Separation Veto}

To avoid effects from not uniquely reconstructed analysis objects, we require a separation in the $(\eta, \phi)$-plane of $\Delta R=\sqrt{\Delta \eta^{2}+\Delta \phi^{2}}>0.4$ between

- lepton - lepton

- lepton - track

- lepton - jet and

- track - jet pairs.

In addition we reject events where

- the missing $E_{T}$ and any jet with $E_{T}>10 \mathrm{GeV}$ are azimuthally separated by less than $\Delta \phi=0.35$. Previous studies in jet20 data[47] have shown a strong correlation of the difference in azimuthal separation and mismeasurement of missing $E_{T}$,

- the missing $E_{T}$ and the leading or next-to-leading lepton are azimuthally separated by less than $\Delta \phi=0.17$. Previous studies have shown that this requirement rejects mismeasurement of missing $E_{T}$ in Drell Yan events.

\subsection{Definition of the Analysis Channels}

\footnotetext{
${ }^{7}$ Exclusive here refers to the exclusivity of the trilepton and dilepton channels within each category; for example the events of the trilepton channel $l_{t} l_{t} l_{t}$ are fully included in the dilepton channel $l_{t} l_{t}$, but the trilepton channels $l_{t} l_{t} l_{t}$ and $l_{t} l_{t} l_{l}$ are exclusive.
} 


\begin{tabular}{|c|c|c|}
\hline Channel & Selection & $E_{T}^{i} / \mathrm{GeV}, i=1,2(, 3)$ \\
\hline \hline \multicolumn{2}{|c|}{ Trilepton channels } \\
\hline$l_{t} l_{t} l_{t}$ & 3 tight leptons or 2 tight leptons $+1 \mathrm{LCE}$ & $15,5,5(8$ if LCE $)$ \\
$l_{t} l_{t} l_{l}$ & 2 tight leptons and $1 \mathrm{CMIO}$ & $15,5,10$ \\
$l_{t} l_{l} l_{l}$ & 1 tight lepton and 2 loose leptons & $20,8,5(10$ if CMIO $)$ \\
\hline Dilepton + track channels & $15,5,5$ \\
\hline$l_{t} l_{t} T$ & 2 tight leptons and 1 isolated track & $20,8(10$ if CMIO $), 5$ \\
\hline & 1 tight and 1 loose lepton and 1 isolated track \\
\hline
\end{tabular}

\begin{tabular}{|c|c|c|}
\hline \multicolumn{2}{|l|}{ Dilepton channels } \\
\hline$l_{t} l_{t}$ & 2 tight leptons & 15,5 \\
$l_{t} l_{l}$ & 1 tight lepton and 1 loose lepton & $20,8(10$ if CMIO $)$ \\
\hline
\end{tabular}

Table 4.6: Definition of exclusive ${ }^{7}$ trilepton and dilepton analysis channels. Dilepton analysis channels are only considered as control regions.

Based on section 4.2 we can reconstruct different analysis objects. As the signature of the leptonic $\tilde{\chi}_{1}^{ \pm} \tilde{\chi}_{2}^{0}$ decay is three leptons and missing transverse energy we define our analysis channels based on the content of main analysis objects, where main analysis objects are TCE and LCE electrons, CMUP/CMX and CMIO muons and isolated tracks. In general the signal to background ratio of events is strongly dependent on the analysis object content, e.g. the signal to background ratio of an event with three TCE electrons is higher than the same ratio for an event with $1 \mathrm{TCE}$ and $2 \mathrm{LCE}$ leptons. The sensitivity of any physics analysis is highly dependent on the signal to background ratio. Assigning the events to different analysis channels based on the signal to background ratio results in a higher sensitivity than having only one channel. Tight leptons are defined as TCE electrons or CMUP/CMX muons; loose leptons are LCE electrons or CMIO muons as these have similar signal to background ratios. Analysis channels are then defined as documented in Table 4.6. To preserve the ability of a simple unification of the results in the five analysis channels into a single result, the analysis channels are defined exclusively: Every event is assigned to the analysis channel with highest signal to background ratio to maximize sensitivity. The 
signal to background fraction of the analysis channels is given in Table 4.10. In addition we require for all analysis channels, that

$$
\left|\sum_{i=1}^{3} q_{i}\right|=1,
$$

where $q_{i}$ is the charge of the selected lepton or isolated track $i, i=1,2,3$. Even though the signature requires that, out of the three selected leptons, there are always two leptons of a kind $\left(e^{+} e^{-}, \mu^{+} \mu^{-}, \tau^{+} \tau^{-}\right)$, we have no requirement on the lepton flavor. Otherwise, as $\tau$ leptons are not identified directly, the case

$$
\tilde{\chi}_{1}^{+} \tilde{\chi}_{2}^{0} \rightarrow \tilde{\chi}_{1}^{0} \mu^{-} \tilde{\chi}_{1}^{0} \tau^{+} \tau^{-} \rightarrow \tilde{\chi}_{1}^{0} \mu^{-} \tilde{\chi}_{1}^{0} e^{+} \bar{\nu}_{e} \nu_{\tau} \pi^{-} \nu_{\tau}
$$

leads to a final state that would not have passed such a lepton flavor requirement.

\subsection{Standard Model and Non-physics Background}

The possible background processes for the trilepton and missing $E_{T}$ signature is dependent on the nature of the analysis objects and thus the analysis channel: the background of the dilepton+track channels is fundamentally different from the background of the trilepton channels.

\subsubsection{Background in the Trilepton Channels}

The major backgrounds for channels with three leptons are

- three genuine leptons from

1. $W Z / \gamma^{*}$

2. $Z / \gamma^{*} Z / \gamma^{*}$

3. $t \bar{t}$ with a subsequent semileptonic B decay

- two genuine leptons and a lepton from a photon conversion from

1. $W W$ and a photon conversion

2. Drell Yan and a photon conversion 
- two genuine leptons and a third lepton from the underlying event ("fake") ${ }^{8}$

The background contribution from processes with three genuine leptons including the processes where one lepton comes from a photon conversion is estimated in Monte Carlo. We weight Monte Carlo events by their respective trigger efficiencies and lepton identification scale factors. The fake contribution is fully estimated in data. For details on the estimation method, see section 4.4.4. As the probability of obtaining a faked lepton is relatively small, the contribution of events with two or three objects being faked is negligible[48].

\subsubsection{Background in the Dilepton + Track Channels}

The major backgrounds for channels with two genuine leptons and one isolated track is

- two genuine leptons and one isolated track from a not fully reconstructed lepton from

1. $W Z / \gamma^{*}$

2. $W W$ and a photon conversion

3. $Z / \gamma^{*} Z / \gamma^{*}$

4. $t \bar{t}$ with a subsequent semileptonic B decay

5. Drell Yan and a photon conversion

- one isolated track from the underlying event or a jet, where one charged particle showers outside the core of the jet, and two genuine leptons from

1. $W Z / \gamma^{*}$

2. $W W$

3. $Z / \gamma^{*} Z / \gamma^{*}$

4. $t \bar{t}$

5. Drell Yan

- two genuine leptons and a third lepton from the underlying event ("fake") ${ }^{9}$

\footnotetext{
${ }^{8}$ Background from processes with one faked lepton is estimated independently of the production process for the two real leptons. However, the major contribution is from Drell Yan.

${ }^{9}$ Backgrounds from processes with one faked lepton are estimated independently of the production process for the two real leptons. However, the major contribution is Drell Yan and $\mathrm{W}+$ jets, where one lepton comes from the $\mathrm{W}$, one lepton is faked by a jet and another jet is identified as an isolated track.
} 


\subsubsection{The Isolated Track Rate}

The isolated track rate[49] is a measure for the probability of getting an additional track from the underlying event. We measure the isolated track rate in $Z \rightarrow e^{+} e^{-}$and $Z \rightarrow \mu^{+} \mu^{-}$ data from the high $p_{T}$ electron and muon triggers as a function of all tracks of a certain quality in the event.

We select $Z \rightarrow e e$ and $Z \rightarrow \mu \mu$ events by requiring two tight electrons or muons with an invariant mass $\left|m_{l l}-91.2 \mathrm{GeV}\right|<15 \mathrm{GeV}$. In addition we require $\mathbb{E}_{T}<10 \mathrm{GeV}$ to remove background with intrinsic $\mathbb{E}_{T}(W Z, t \bar{t})$. The remaining background is below 1\%[50]. The ratio of the number of events with at least one isolated track and the number of all events with a certain number of tracks (excluding the two tracks forming the $\mathrm{Z}$ mass) is defined as the isolated track rate. We reconstruct tracks based on the requirements in Table 4.2, where the isolation requirement is applied to isolated tracks only.

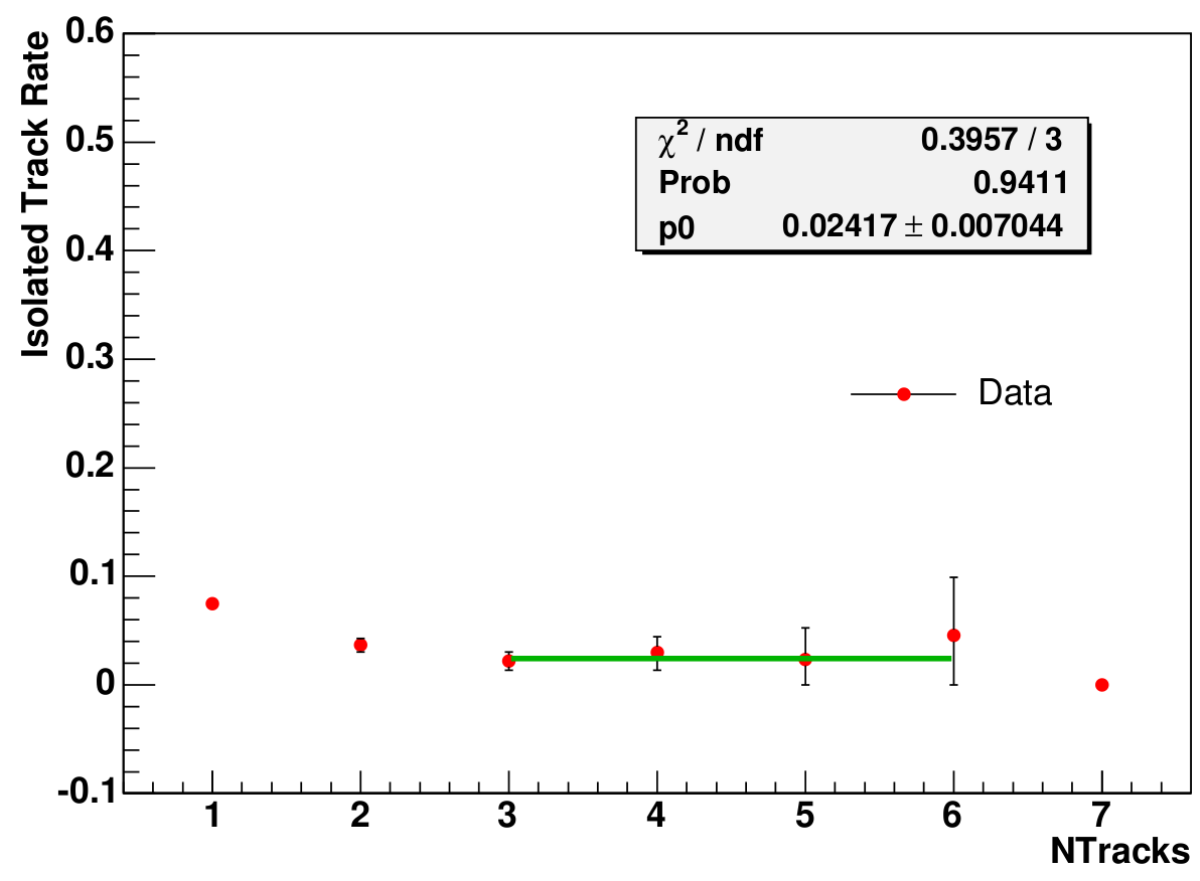

Figure 4.7: Measurement of the isolated track rate in $\mathrm{Z}$ events 
Figure 4.7 shows the results of this measurement. For NTracks $>2$ we assume that the isolated track rate is constant and fit a function of the form $\operatorname{ITR}(\mathrm{NTracks})=p_{0}$. We then obtain the isolated track rate

$$
\operatorname{ITR}(\text { NTracks })=\left\{\begin{array}{ll}
0.075 \pm 0.005 & \text { for NTracks }=1 \\
0.037 \pm 0.006 & \text { for NTracks }=2 \\
0.024 \pm 0.007 & \text { for NTracks }>2
\end{array} .\right.
$$

The isolated track rate is applied to events in Monte Carlo if there are two identified leptons and every track is matched to one of the generator-level leptons. Together with the trigger efficiency and the lepton ID scale factor the isolated track rate forms the event weight $\varepsilon_{\text {dilep}}$. As the isolated track rate does not include any information about of the additional track (e.g. angles to other tracks), it is not possible to apply the usual cuts to this type of events. We assume that the ratio of events that pass the cut to all events with two genuine lepton and one isolated track from the underlying event is equal to the equivalent ratio for events with two leptons and a track, that is not from the underlying event. This ratio is denoted by $N_{\text {dilep+track }}^{\text {cut }} / N_{\text {dilep+track }}^{\text {base }}$, where $N_{\text {dilep+track }}^{\text {base }}$ is the number of events before and $N_{\text {dilep+track }}^{\text {cut }}$ the number of events after cuts. If we denote the number of dilepton events that are missing a track by $N_{\text {dilep}}$, the average event weight for dilepton + track events by $\varepsilon_{\text {dilep+track }}^{\text {cut }}$ and the total number of events by $N_{\text {gen }}$, the overall event acceptance is

$$
A=\frac{N_{\text {dilep }+ \text { track }}^{\text {cut }} \varepsilon_{\text {dilep }+ \text { track }}^{\text {cut }}+N_{\text {dilep }+ \text { track }}^{\text {cut }} / N_{\text {dilep }+ \text { track }}^{\text {base }} N_{\text {dilep }} \varepsilon_{\text {dilep }}}{N_{\text {gen }}} .
$$

\subsubsection{The Fake Rate}

The expected number of events, where two leptons come from a physics process and third lepton from underlying event, is estimated with the so-called fake rate[51]. The fake rate is a measure for the probability of a jet being reconstructed as an electron or a track being reconstructed as a muon. In addition the fake rate includes real leptons within jets from processes such as a semileptonic B decay.

In jet-triggered samples jets, isolated tracks, electrons and muons are reconstructed. For the electron fake rate we count the number of jets and the number of reconstructed leptons, which are within $\Delta R=\sqrt{\Delta \eta^{2}+\Delta \phi^{2}}<0.4$ of any reconstructed jet, as a function of the 

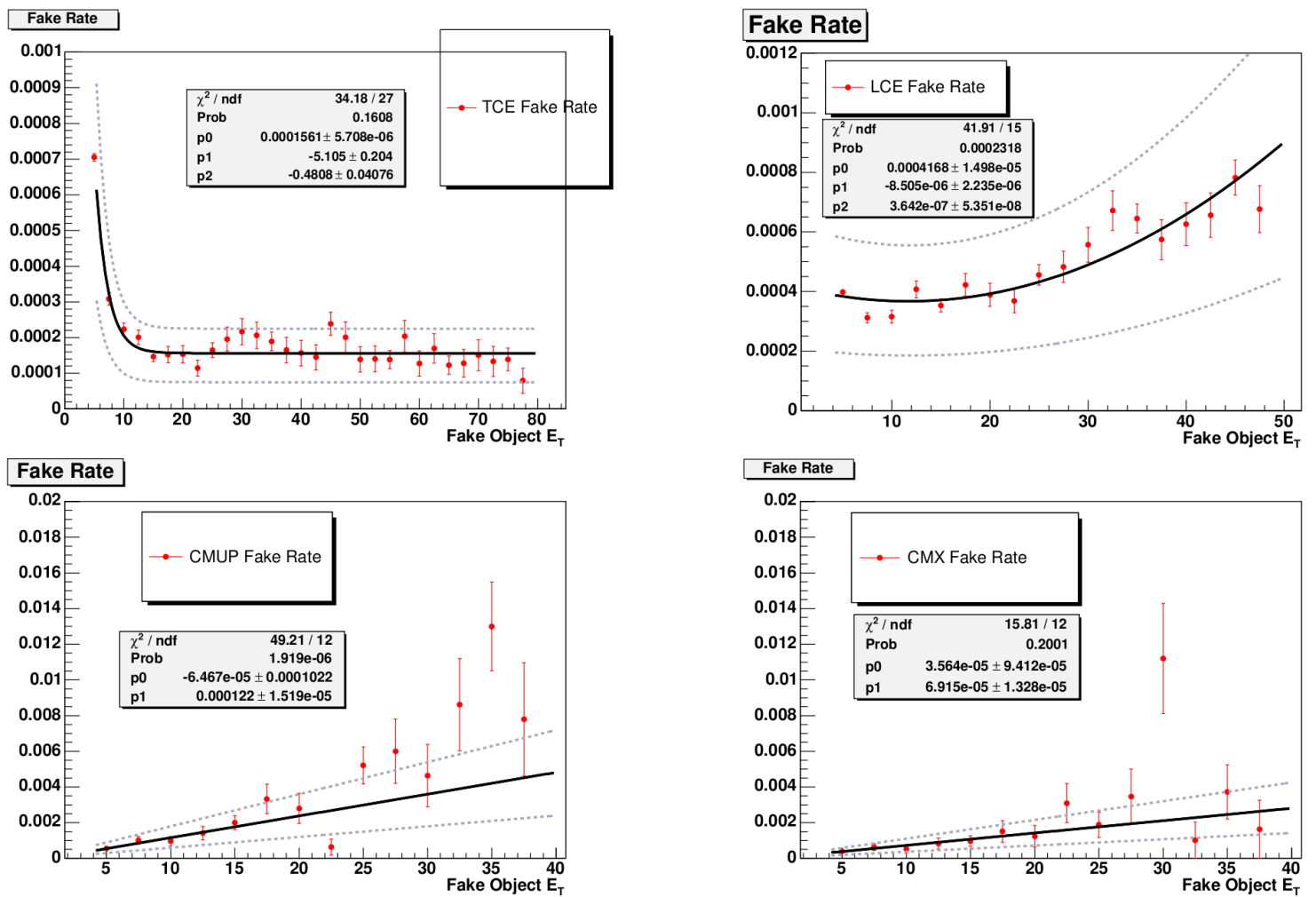

\section{Fake Rate}

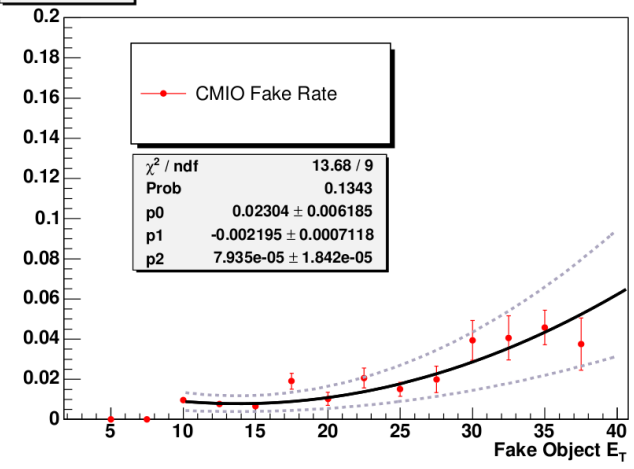

Figure 4.8: Fake rates for TCE, LCE, CMUP, CMX and CMIO leptons. We fit an exponential added to a constant to the fake rates for TCE, LCE and CMIO and first order polynomials to the fake rates for CMUP and CMX. The dashed lines show the $50 \%$ systematic error. 
jet's $E_{T}$. The electron fake rate is defined as the ratio of counted leptons and the number of all jets as a function of jet $E_{T}$. The muon fake rate is determined in a similar way; unlike for electrons where jets have the ability to fake, for muons isolated tracks have the ability to fake. The fake rate is defined accordingly. A constant and an exponential of the form

$$
f(x)=p_{0}+\exp \left(p_{1} \times E_{T}+p_{2}\right)
$$

are fit to the fake rates of TCE, LCE and CMIO leptons independently. To CMUP and CMX muon fake rates we fit a first order polynomial of the form

$$
f(x)=p_{0}+p_{1} x
$$

The results are shown in Fig. 4.8.

The fake rates are then applied to dilepton events in data where a fakeable object exists. According to the measurement of the fake rate a fakeable object is a jet for electrons and an isolated track for muons. Similar to the application of the isolated track rate the fake rate is used as a component of the event weight.

\subsection{Control and Signal Regions}

This analysis is set up as an unbiased counting experiment. We define signal and control regions. Before the data in the signal region is examined, we verify our background predictions in the control regions.

\subsubsection{Definition}

In order the check the estimation methods several control regions are defined. The signal signature is expected to have high $\mathbb{E}_{T}$, so that we demand $\mathbb{E}_{T}>15 \mathrm{GeV}$ for the signal region. In addition we define our signal region outside a window of $15 \mathrm{GeV}$ around the $\mathrm{Z}$ mass, where $m_{O S}^{1} \in[76 \mathrm{GeV}, 106 \mathrm{GeV}]$ and $m_{O S}^{1}$ is the higher of the two invariant masses that can be formed from the three analysis objects. In this region we expect the observed

events to be dominated by background from Drell Yan. We use the regions where $m_{O S}^{1} \in$ [76 GeV, $106 \mathrm{GeV}]$ or $\mathbb{E}_{T}<15 \mathrm{GeV}$ as control regions. The control regions are further split into the analysis channels of Table 4.6. The control region where $m_{O S}^{1} \in[76 \mathrm{GeV}, 106 \mathrm{GeV}]$ 


\section{Control and Signal Regions}

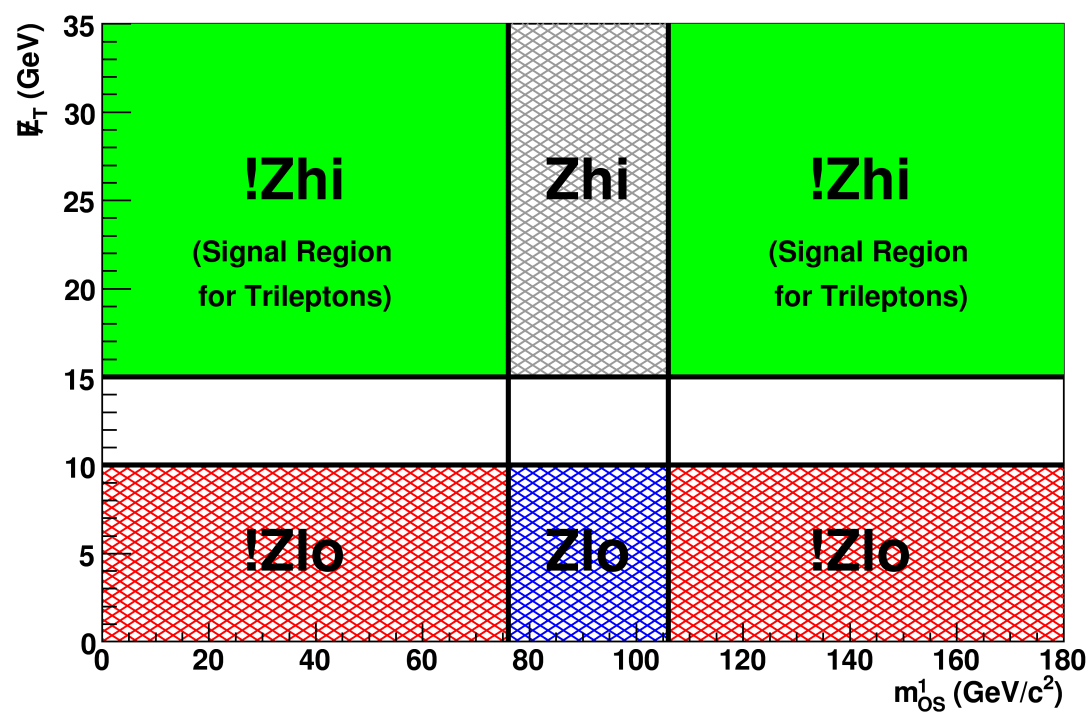

Figure 4.9: Illustration to the definition of signal and control regions. !Zhi is the signal region for trilepton events; for dilepton events !Zhi is a control region. $m_{O S}^{1}$ is the higher of the two invariant masses that can be calculated from three analysis objects

is used to check the estimations for Drell Yan; the region where $\mathbb{E}_{T}<10 \mathrm{GeV}$ is used to check low mass Drell Yan. Compared to the number of events with two leptons the number of events with three leptons is relatively low. To check the predictions in regions with high statistics we use events with two leptons as control regions. We split them into channels with two tight leptons and one tight and one loose lepton. The control region with two leptons and high missing $E_{T}$ can be used to test the predictions for $t \bar{t}$. Additionally we use control regions based on the lepton flavor of the final states. The naming scheme and the exact definition of control and signal regions can be found in Table 4.7.

\subsubsection{Background Estimation}

We estimate the Standard Model and non-physics background with exception of the fake contribution in the Monte Carlo samples listed in Table 3.1. The predictions can be found in appendix A. In Figs. 4.10 and 4.11 the ratio of the difference of observed and expected and the expected number of event for the control regions defined in Table 4.7 is plotted. If the predictions are consistent with the observations, the plotted ratio should agree with 0 within its limits. 


\begin{tabular}{|c|c|c|}
\hline Region & \multicolumn{2}{|l|}{ Definition } \\
\hline \multicolumn{3}{|c|}{ Trilepton Control Regions } \\
\hline Signal & $\mathbb{E}_{T}>15 \mathrm{GeV}$ & $m_{O S}^{1} \notin[76 \mathrm{GeV}, 106 \mathrm{GeV}]$ \\
\hline Zlo & $\mathbb{E}_{T}<10 \mathrm{GeV}$ & $m_{O S}^{1} \in[76 \mathrm{GeV}, 106 \mathrm{GeV}]$ \\
\hline !Zlo & $\mathbb{E}_{T}<10 \mathrm{GeV}$ & $m_{O S}^{1} \notin[76 \mathrm{GeV}, 106 \mathrm{GeV}]$ \\
\hline !Zhi & $\mathbb{E}_{T}>15 \mathrm{GeV}$ & $m_{O S}^{1} \notin[76 \mathrm{GeV}, 106 \mathrm{GeV}]$ \\
\hline \multicolumn{3}{|c|}{ Dilepton Control Regions } \\
\hline $\mathrm{Z}$ & & $m_{l l} \in[76 \mathrm{GeV}, 106 \mathrm{GeV}]$ \\
\hline Zlo & $\mathbb{E}_{T}<10 \mathrm{GeV}$ & $m_{l l} \in[76 \mathrm{GeV}, 106 \mathrm{GeV}]$ \\
\hline Zhi & $\mathbb{E}_{T}>15 \mathrm{GeV}$ & $m_{l l} \in[76 \mathrm{GeV}, 106 \mathrm{GeV}]$ \\
\hline !Z & & $m_{l l} \notin[76 \mathrm{GeV}, 106 \mathrm{GeV}]$ \\
\hline !Zlo & $\mathbb{E}_{T}<10 \mathrm{GeV}$ & $m_{l l} \notin[76 \mathrm{GeV}, 106 \mathrm{GeV}]$ \\
\hline !Zhi & $\mathbb{E}_{T}>15 \mathrm{GeV}$ & $m_{l l} \notin[76 \mathrm{GeV}, 106 \mathrm{GeV}]$ \\
\hline loMet & $\mathbb{E}_{T}<10 \mathrm{GeV}$ & \\
\hline
\end{tabular}

Table 4.7: Definition of control and signal regions. For the trilepton control and signal regions, additionally we require $\left|\sum_{i=1}^{3} q_{i}\right|=1$, where $q_{i}$ is the charge of analysis object $\mathrm{i}$.

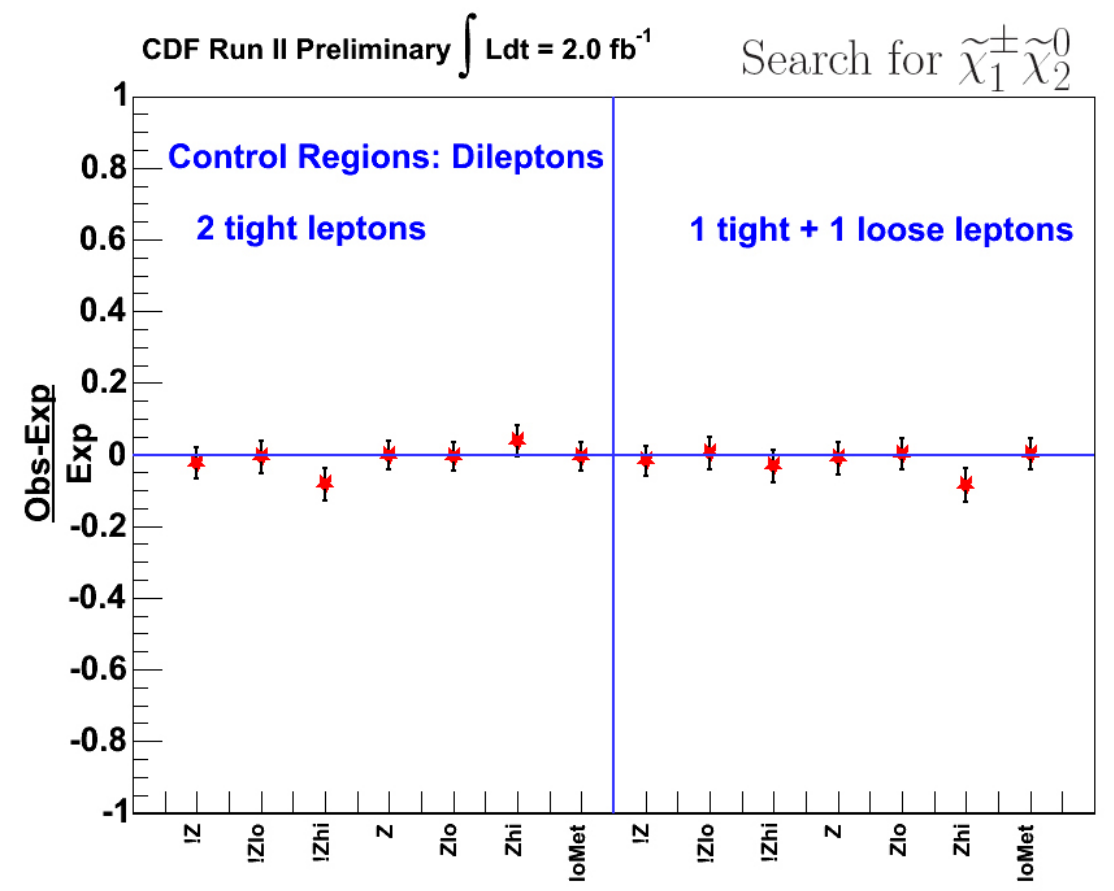

Figure 4.10: Comparison of expected and observed number of events for the dilepton control regions 


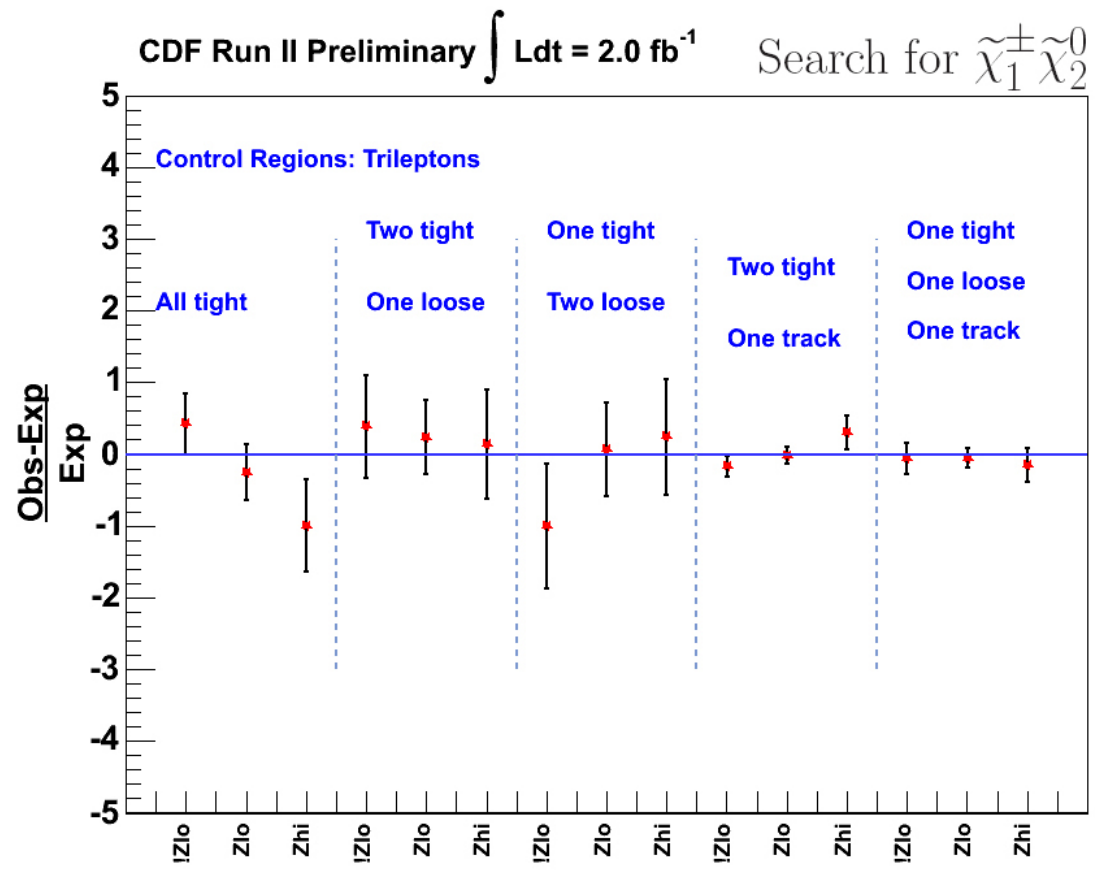

Figure 4.11: Comparison of expected and observed number of events for the trilepton control regions

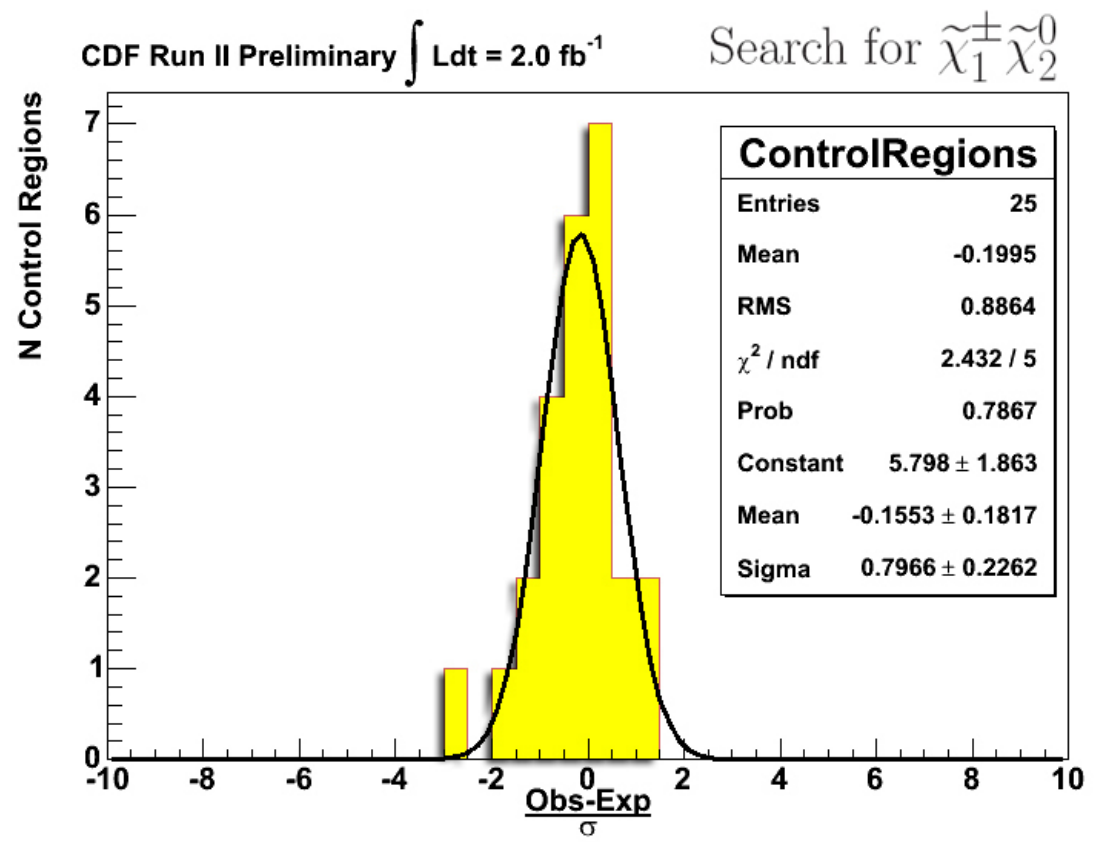

Figure 4.12: Summary of expected and observed number of events in all control regions. To check the number of events in the individual control regions for gaussian distribution with $\mu=\operatorname{Exp}$ and $\sigma^{2}=\operatorname{Exp}$ we fit a function of the form $C \cdot \mathcal{N}\left(\mu, \sigma^{2}\right)$, where $\mu, \sigma^{2}$ and $C$ are the fit parameters. 
Following the law of rare events we assume that the numbers of observed events follow a Poisson inspired normal distributions $\mathcal{N}(\operatorname{Exp}, \operatorname{Exp})$ with a density of the form

$$
f(x)=\frac{1}{\sqrt{2 \pi \operatorname{Exp}}} \exp \left(-\frac{1}{2} \frac{(x-\operatorname{Exp})^{2}}{\operatorname{Exp}}\right),
$$

where Exp is the number of expected events. As the mean $\mu=$ Exp and the variance $\sigma^{2}=\operatorname{Exp}$ is different for every analysis channels, we plot $($ Obs $-\operatorname{Exp}) / \sqrt{\operatorname{Exp}}$, where Obs denotes the number of observed events in data, in a histogram in Fig. 4.12. Assuming the expected number of events is in fact a good background estimation, the histogram should follow a $\mathcal{N}(0,1)$ distribution. We check this by fitting the appropriate density function with a normalization factor to account for the fact, that the histogram is not normalized and obtain

$$
\begin{aligned}
& \mu_{\text {fit }}=-0.16 \pm 0.18, \\
& \sigma_{\text {fit }}=0.78 \pm 0.23
\end{aligned}
$$

which is consistent with the assumption of a $\mathcal{N}(0,1)$ distribution.

In addition to the comparison of expected and observed number of events we check distributions of $p_{T}^{1}, p_{T}^{2}, E_{T}^{1}, E_{T}^{2}, \Delta \phi, m_{l l}, \mathbb{E}_{T}, N_{\text {jets }}, N_{\text {tracks }}$ in each dilepton control region split into the analysis channels $l_{t} l_{t}$ and $l_{t} l_{l}$. In the trilepton control regions we check the distribution of $p_{T}^{1}, p_{T}^{2}, p_{T}^{3}, E_{T}^{1}, E_{T}^{2}, E_{T}^{3}, \Delta \phi_{12}, \Delta \phi_{13}, \Delta \phi_{23}, m_{T}^{1}, m_{T}^{2}, m_{T}^{3}, m_{O S}^{1}, m_{O S}^{2}, \mathbb{E}_{T}$, $N_{\text {jets }}$ in each analysis channel. A selection of control region plots can be seen in Figs. 4.13 to 4.16; a more complete set of control region plots can be found in [52].

\subsection{Predictions and Results for the Signal Region}

\subsubsection{Signal Optimization}

For the signal region we define additional cuts to optimize the analysis channels for a better signal to background ratio. In the following we list the optimization cuts and the backgrounds which are most affected by the cut:

- $\mathbb{E}_{T} \geq 20 \mathrm{GeV}$ to remove Drell Yan which has no intrinsic $\mathbb{E}_{T}$ 


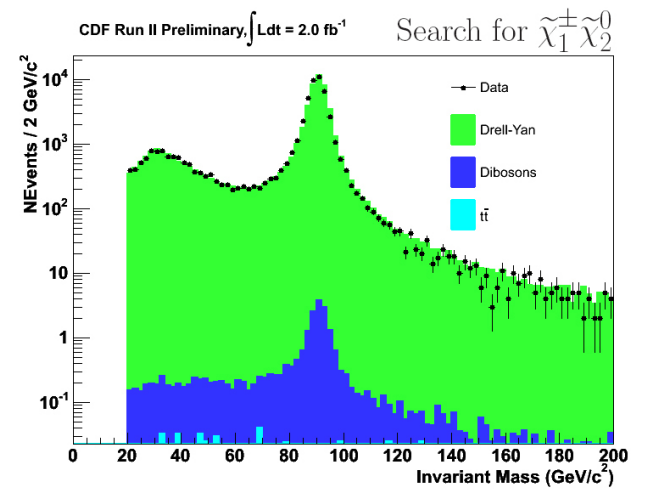

Figure 4.13: Invariant mass of the two leptons in the $l_{t} l_{t}$ analysis channel of the loMet dilepton control region

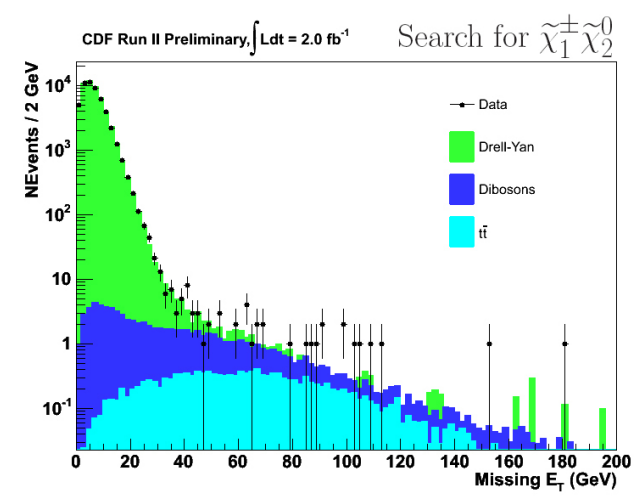

Figure 4.15: Missing transverse energy of events in the $l_{t} l_{t}$ analysis channel of the $\mathrm{Z}$ dilepton control region

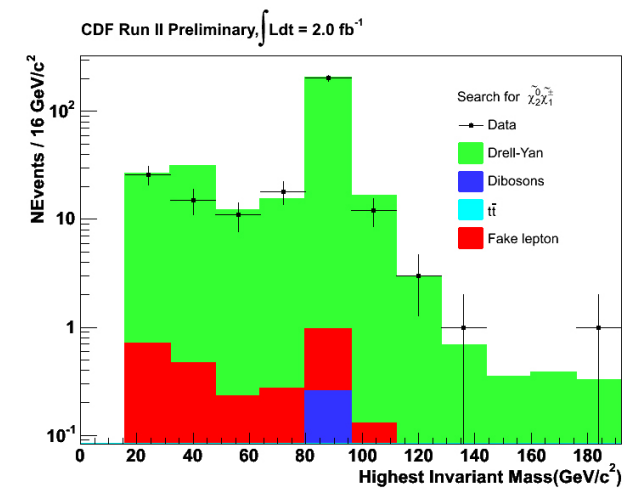

Figure 4.14: Invariant mass of the two leptons in the $l_{t} l_{t} T$ analysis channel of the loMet trilepton control region

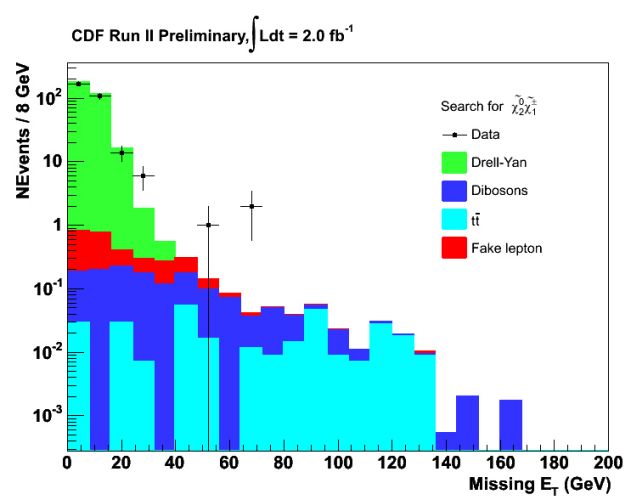

Figure 4.16: Missing transverse energy of events in the $l_{t} l_{t} T$ analysis channel of the $\mathrm{Z}$ trilepton control region 
- $\Delta \phi_{O S}^{i} \leq \begin{cases}2.9 & \text { for the dilepton }+ \text { track channels } \\ 2.8 & \text { for the trilepton channel }\end{cases}$

and $i=1,2$ to remove Drell Yan, where the leptons have the tendency to be back-toback.

- $m_{O S}^{i} \notin[76 \mathrm{GeV}, 106 \mathrm{GeV}]$ for $i=1,2$ to remove on-shell Drell Yan and diboson background

- $N_{\text {jets }}<2$ and $\sum_{\text {jets }} E_{T, i} \leq 80 \mathrm{GeV}$, where the sum goes over jets with $E_{T}^{\text {jet }}>10 \mathrm{GeV}$, to remove $\mathrm{QCD}$, especially $t \bar{t}$, background where hadronic activity is expected. The leptonic decay of chargino and neutralino does not produce hard jets.

The background estimation for the signal region based on Monte Carlo (data for the fake category) and optimized cuts for the supersymmetric signal can be found in Table 4.8.

\begin{tabular}{|l|ccccc|}
\hline \multicolumn{1}{|c|}{$\begin{array}{c}\text { Background } \\
\text { Process }\end{array}$} & $l_{t} l_{t} l_{t}$ & $l_{t} l_{t} l_{l}$ & $l_{t} l_{l} l_{l}$ & $l_{t} l_{t} T$ & $l_{t} l_{l} T$ \\
\hline \hline$Z \rightarrow e e$ & 0.03 & 0.00 & 0.00 & 0.81 & 0.73 \\
$Z \rightarrow \mu \mu$ & 0.02 & 0.01 & 0.00 & 0.00 & 0.30 \\
$Z \rightarrow \tau \tau$ & 0.00 & 0.00 & 0.00 & 0.82 & 0.29 \\
$W W$ & 0.00 & 0.00 & 0.00 & 0.38 & 0.29 \\
$W Z$ & 0.24 & 0.13 & 0.06 & 0.15 & 0.05 \\
$Z Z$ & 0.05 & 0.07 & 0.02 & 0.08 & 0.04 \\
$t \bar{t}$ & 0.02 & 0.01 & 0.03 & 0.22 & 0.18 \\
Fake & 0.12 & 0.04 & 0.03 & 0.75 & 0.41 \\
\hline$\sum$ backgrounds & $0.49 \pm 0.07$ & $0.25 \pm 0.03$ & $0.14 \pm 0.22$ & $3.22 \pm 0.60$ & $2.28 \pm 0.51$ \\
\hline Signal at BP1 & $2.25 \pm 0.17$ & $1.61 \pm 0.13$ & $0.68 \pm 0.08$ & $4.44 \pm 0.22$ & $2.42 \pm 0.16$ \\
\hline
\end{tabular}

Table 4.8: Number of expected signal and background events for $\int \mathcal{L} d t=2.0 \mathrm{fb}^{-1}$ of data. Uncertainties are statistical and partial systematics. The boson background processes include off-shell bosons.

\subsubsection{Systematic Error}

The systematic errors used for this analysis are listed in Table 4.9, where 

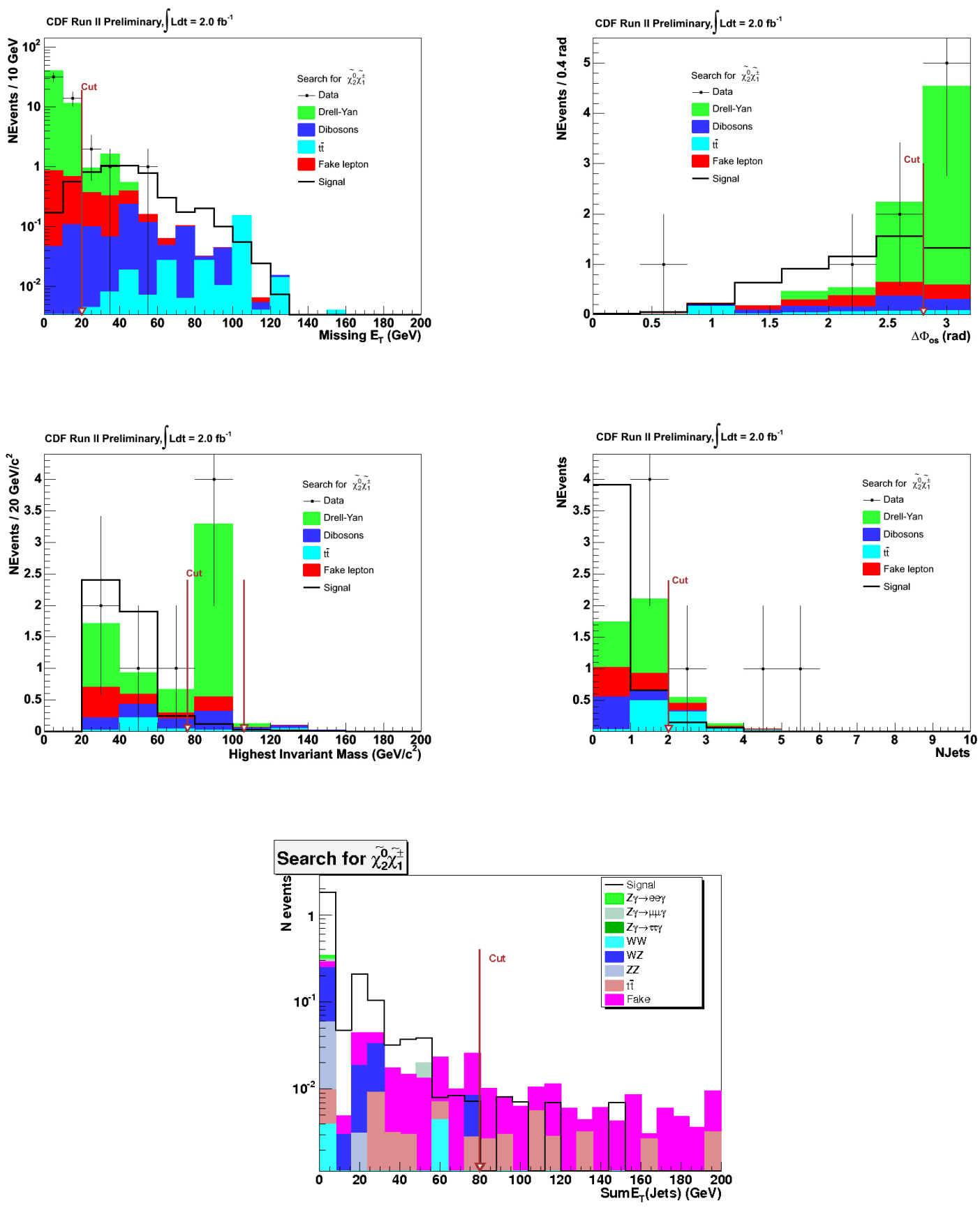

Figure 4.17: Signal and background N-1 plots for the $\mathbb{E}_{T}, \Delta \phi_{O S}^{i}, m_{O S}^{i}$ and the $N_{\text {jets }}$ cuts in the analysis channel $l_{t} l_{t} T$ (for the $\sum_{\text {jets }} E_{T, i}$ cut in the channel $l_{t} l_{t} l_{t}$ ). The backgrounds are stacked. We apply all cuts except the cut on the quantity plotted; $N_{\text {jets }}$ and $\sum_{\text {jets }} E_{T, i}$ are highly correlated and we plot N-2 plots, where the cuts of both quantities are not applied. 


\begin{tabular}{|c|ccccc|c|}
\hline \multirow{2}{*}{ Source } & \multicolumn{6}{|c|}{ Channels } \\
& $l_{t} l_{t} l_{t}$ & $l_{t} l_{t} l_{l}$ & $l_{t} l_{l} l_{l}$ & $l_{t} l_{t} T$ & $l_{t} l_{l} T$ & Signal at BP1 \\
\hline \hline ID & 2.3 & 2.5 & 2.2 & 1.8 & 1.8 & 4 \\
Trig & 0.3 & 0.3 & 0.3 & 0.2 & 0.2 & 0.5 \\
JES & 1.5 & 1.7 & 3.5 & 3.9 & 5.2 & 0.5 \\
X-sec & 5.0 & 5.9 & 5.0 & 2.3 & 2.4 & 10 \\
PDF & 1.4 & 1.6 & 1.3 & 1.5 & 1.5 & 2 \\
ISR/FSR & 2.3 & 2.5 & 2.2 & 1.8 & 1.8 & 4 \\
Conv & 2.2 & 2.1 & 1.8 & - & - & - \\
ITR (nom) & - & - & - & 5.8 & 8.6 & - \\
ITR (alt) & - & - & - & 6.0 & 10.5 & - \\
Fake & 12.2 & 8 & 10.7 & 11.6 & 9.0 & - \\
\hline
\end{tabular}

Table 4.9: Systematic error broken down by source and channel in percentage. A universal $6 \%$ uncertainty on the luminosity is not included.

ID is the error of th lepton identification scale factors described in the appropriate CDF notes.

Trig is the error of the trigger efficiencies[25].

JES is the error on the Jet Energy Scale. The jet energies are fluctuated up and down by one standard deviation and the difference in acceptance from the nominal is evaluated. In cases where the statistics preclude this type of estimation we use a signal-like selection (two lepton, $\mathbb{E}_{T}>20 \mathrm{GeV}, \sum_{\text {jets }} E_{T, i}<80 \mathrm{GeV}, N_{\text {jets }}<2$ and $\Delta \phi_{12}<2.9$ ) and get the difference from the nominal.

$\mathrm{X}$-sec is the error on the cross section of the background processes. For diboson processes we use the error from the CDF WZ search[53]; for $t \bar{t}$ we use the error from the top mass measurement[54].

PDF is the error of the Parton Distribution Function. We use the errors quoted by [53, 54].

ISR/FSR is the error due to turning on Initial State (ISR) and Final State Radiation (FSR). We use the measurements from a previous round of this analysis. 
Conv is the systematic error on the conversion scale factor. The systematic error from [46] is applied to the backgrounds in trilepton channels where the third lepton is expected to come from a photon conversion, e.g. $Z \gamma \rightarrow e e \gamma$.

ITR (nom) is the systematic on the isolated track rate as described in section 4.4.3.

ITR (alt) is the difference between the ITR parameterized as a function of number of tracks and the ITR parameterized as a function of $\sum_{\text {jet }} E_{T, i}$ where the sum goes over all jets with $E_{T}^{\text {corr }}>10 \mathrm{GeV}$.

Fake is the systematic error on the fake measurement which is taken to be $50 \%$.

\subsubsection{Results}

\begin{tabular}{|c|ccccc|c|}
\hline Channel & $l_{t} l_{t} l_{t}$ & $l_{t} l_{t} l_{l}$ & $l_{t} l_{l} l_{l}$ & $l_{t} l_{t} T$ & $l_{t} l_{l} T$ & Echannels \\
\hline \hline Signal at BP1 & 2.25 & 1.61 & 0.68 & 4.44 & 2.42 & 11.40 \\
Statistical Uncertainty & \pm 0.13 & \pm 0.11 & \pm 0.07 & \pm 0.19 & \pm 0.14 & \pm 0.30 \\
Systematic Uncertainty & \pm 0.29 & \pm 0.21 & \pm 0.09 & \pm 0.58 & \pm 0.32 & \pm 0.76 \\
\hline Background & 0.49 & 0.25 & 0.14 & 3.22 & 2.28 & 6.38 \\
Statistical Uncertainty & \pm 0.04 & \pm 0.03 & \pm 0.02 & \pm 0.48 & \pm 0.47 & \pm 0.67 \\
Systematic Uncertainty & \pm 0.08 & \pm 0.03 & \pm 0.02 & \pm 0.53 & \pm 0.42 & \pm 0.68 \\
\hline Observed & 1 & 0 & 0 & 4 & 2 & 7 \\
\hline
\end{tabular}

Table 4.10: Final number of expected signal and background events in the different analysis channels together with the observed number of events in data. The signal predictions are for benchmark point BP1.

Table 4.10 shows the observed number of events in the signal region in comparison with the expected number based on estimations in Monte Carlo and data. The observed number of events shows no significant excess over the expected number of events in the sum of all channels as well as in every individual channel. A summary of the characteristics of the observed events can be found in Table 4.11. The number of events at the mSUGRA benchmark point BP1 is significantly higher than the expected background. This shows that the analysis is sensitive to mSUGRA. A more detailed analysis of the sensitivity can be found in section 5 . 


\begin{tabular}{|c|c|c|ccc|cc|c|c|}
\hline Channel & Date & Type & $E_{T}^{1}$ & $E_{T}^{2}$ & $E_{T}^{3}$ & $M_{O S}^{1}$ & $M_{O S}^{1}$ & $\mathbb{E}_{T}$ & Jet $E_{T}$ \\
\hline \hline$l_{t} l_{t} l_{t}$ & Aug 8, 2005 & -TCE +TCE -TCE & 23.6 & 17.2 & 5.8 & 29.1 & 15.5 & 37.2 & 59.4 \\
\hline$l_{t} l_{t} T$ & Oct 25, 2006 & -TCE + TCE -TRK & 26.9 & 9.7 & 8.5 & 41.4 & 18.8 & 27.6 & 23.6 \\
$l_{t} l_{t} T$ & Mar 12, 2007 & -TCE -TCE +TRK & 22.8 & 9.3 & 55.9 & 70.3 & 46.2 & 57.8 & 17.7 \\
$l_{t} l_{t} T$ & Nov 14, 2006 & +CMUP -CMX -TRK & 33.7 & 6.2 & 9.2 & 32.9 & 28.3 & 20.4 & 21.4 \\
$l_{t} l_{t} T$ & Feb 7, 2006 & -CMUP +CMX -TRK & 44.7 & 21.2 & 7.8 & 29.2 & 25.8 & 38.9 & 41.1 \\
\hline$l_{t} l_{l} T$ & Feb 20, 2005 & +CMUP -CMIO +TRK & 22.8 & 12.2 & 6.5 & 39.2 & 17.8 & 28.5 & 33.6 \\
$l_{t} l_{l} T$ & Jan 24, 2007 & +CMUP -CMIO -TRK & 58.6 & 69.9 & 44.1 & 124.0 & 57.5 & 36.8 & - \\
\hline
\end{tabular}

Table 4.11: Characteristics of the observed events. $E_{T}^{i}, i=1,2,3$ refers to transverse energy for electrons and transverse momentum for muons and tracks; $M_{O S}^{i}, i=1,2$ are the invariant masses of the two oppositely charged lepton combinations. All numbers are in GeV. 


\section{Chapter 5}

\section{Interpretation of the Results in the mSUGRA Model}

In the following a systematic application of the results of the analysis to the supersymmetric model mSUGRA (minimal supergravity grand unification) is tried. It was chosen to evaluate the results of this analysis in mSUGRA as this model is widely accepted as a benchmark for supersymmetry searches[55, 56]. As discussed in section 2.2.4 mSUGRA has four undetermined scalar parameters and one undetermined sign:

- $m_{0}$, the common scalar mass at the GUT scale,

- $m_{1 / 2}$, the common gaugino mass at the GUT scale,

- $\tan \beta$, the ratio of the vacuum expectation values of the two Higgs doublets,

- $A_{0}$, the common trilinear coupling constant at the GUT scale,

- $\operatorname{sgn} \mu$, the sign of $\mu$, where $\mu$ is the Higgs mixing parameter.

Based on different phenomenology classes a set of three benchmark points is defined in Table 5.1. Benchmark point BP1 was used to evaluate the analysis in section 4.

The ability of this analysis to exclude a distinct set of parameters depends on several factors, among which the most important are:

- Kinematics of the event. As described in section 4.3 the most important cuts for the analysis are minimum transverse momentum requirements for the three leptons (two leptons and one track), a minimum missing transverse energy requirement and the requirement that the invariant mass of a pair of oppositely charged leptons has to be outside a certain interval around the mass of the $Z^{0}$ boson. The set of mSUGRA parameters can have an important effect on the transverse momentum of the leptons. 


\begin{tabular}{|c|c|c|c|}
\hline & BP1 & BP2 & BP3 \\
\hline \hline$m_{0} / \mathrm{GeV} / \mathrm{c}^{2}$ & 60 & 140 & 110 \\
\hline$m_{1 / 2} / \mathrm{GeV} / \mathrm{c}^{2}$ & 190 & 190 & 190 \\
\hline $\tan \beta$ & 3 & 3 & 3 \\
\hline$A_{0} / \mathrm{GeV}$ & 0 & 0 & 0 \\
\hline $\operatorname{sgn} \mu$ & 1 & 1 & -1 \\
\hline \hline$m\left(\tilde{\chi}_{1}^{0}\right) / \mathrm{GeV} / \mathrm{c}^{2}$ & 67.8 & 68.4 & 77.9 \\
\hline$m\left(\tilde{\chi}_{2}^{0}\right) / \mathrm{GeV} / \mathrm{c}^{2}$ & 124.4 & 125.7 & 152.3 \\
\hline$m\left(\tilde{\chi}_{1}^{ \pm}\right) / \mathrm{GeV} / \mathrm{c}^{2}$ & 121.9 & 123.3 & 152.7 \\
\hline$m\left(\tilde{e}_{R}^{ \pm}\right)=m\left(\tilde{\mu}_{R}^{ \pm}\right) / \mathrm{GeV} / \mathrm{c}^{2}$ & 99.8 & 161.1 & 135.9 \\
\hline$m\left(\tilde{\tau}_{1}^{ \pm}\right) / \mathrm{GeV} / \mathrm{c}^{2}$ & 99.7 & 160.6 & 135.9 \\
\hline$m\left(\tilde{\nu}_{e}\right)=m\left(\tilde{\nu}_{\mu}\right) / \mathrm{GeV} / \mathrm{c}^{2}$ & 129.9 & 180.7 & 159.0 \\
\hline$m\left(\tilde{\nu}_{\tau}\right) / \mathrm{GeV} / \mathrm{c}^{2}$ & 128.8 & 180.1 & 158.2 \\
\hline
\end{tabular}

Table 5.1: Definition of the mSUGRA benchmark points and selected masses

- Cross section for the associated production of a chargino-neutralino pair in a $p \bar{p}$ collision $\sigma\left(\tilde{\chi}_{2}^{0} \tilde{\chi}_{1}^{ \pm}\right)$

- Branching ratio of chargino and neutralino into 3 leptons $\operatorname{BR}\left(\tilde{\chi}_{2}^{0} \tilde{\chi}_{1}^{ \pm} \rightarrow 3\right.$ leptons $)$

- the ratio of the number of events in a channel with three leptons and the number of events in a channel with two leptons and a track; a good quantity to study this ratio is the mean number of $\tau$ leptons per event ${ }^{1}$ as signal tracks dominantly come from hadronic $\tau$ decays $^{2}$.

\footnotetext{
${ }^{1}$ See appendix $\mathrm{C}$ for details.

${ }^{2}$ The single-prong branching fraction for the $\tau$ lepton is $85.33 \%$, among which $17.36 \%$ is the decay to $\mu \bar{\nu}_{\mu} \nu_{\tau}$ and $17.84 \%$ is the decay to $e \bar{\nu}_{e} \nu_{\tau}[1]$.
} 


\subsection{Effects of the mSUGRA Parameters}

In the following we investigate the effect of a change of the mSUGRA parameters. We start at benchmark point BP1 with $m_{0}=60 \mathrm{GeV} / \mathrm{c}^{2}, m_{1 / 2}=190 \mathrm{GeV} / \mathrm{c}^{2}, \tan \beta=3, A_{0}=$ $0 \mathrm{GeV}, \mu>0$ and vary one parameter at a time. For $\mu<0$ we start at BP3 $m_{0}=$ $110 \mathrm{GeV} / \mathrm{c}^{2}, m_{1 / 2}=190 \mathrm{GeV} / \mathrm{c}^{2}, \tan \beta=3, A_{0}=0 \mathrm{GeV}$ to maximize the sensitivity of the analysis. The mass of the important supersymmetric particles, the branching ratio of the different decay channels, the branching ratio into three leptons split into final states with 0,1,2 or $3 \tau$ leptons and the cross section for chargino-neutralino production are shown.

\subsubsection{The Common Scalar Mass $m_{0}$}
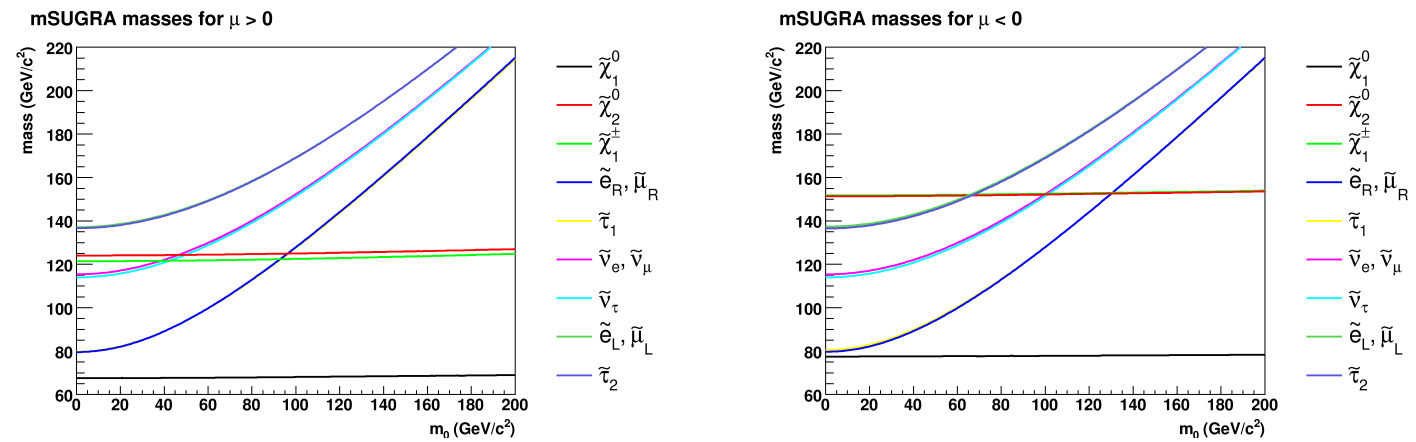

Figure 5.1: Mass of the relevant supersymmetric particles as a function of $m_{0}$ at benchmark point BP1 with $\mu>0$ (left) and at benchmark point BP3 with $\mu<0$ (right). Benchmark point $\mathrm{BP} 1$ is at $m_{0}=60 \mathrm{GeV} / \mathrm{c}^{2}$ for $\mu>0$, benchmark point $\mathrm{BP} 2$ is at $m_{0}=140 \mathrm{GeV} / \mathrm{c}^{2}$ for $\mu>0$ and benchmark point BP3 is at $m_{0}=110 \mathrm{GeV} / \mathrm{c}^{2}$ for $\mu<0$.

It can be seen in Fig. 5.1, that the mass of the neutralino and chargino is almost independent of $m_{0}$, while the mass of the charged sleptons and the sneutrinos has a dependence on $m_{0}$. As long as the mass of the sleptons is lower than the mass of the decaying neutralino and chargino a sequential two-body decay via on-shell sleptons

$$
\tilde{\chi}_{1}^{ \pm} \rightarrow \tilde{l}_{R}^{ \pm} \nu \rightarrow \tilde{\chi}_{1}^{0} l^{ \pm} \nu, \quad \tilde{\chi}_{2}^{0} \rightarrow \tilde{l}_{R}^{ \pm} l^{\mp} \rightarrow \tilde{\chi}_{1}^{0} l^{+} l^{-}
$$

where $\tilde{l}_{R}^{ \pm}=\tilde{e}_{R}^{ \pm}, \tilde{\mu}_{R}^{ \pm}, \tilde{\tau}_{1}^{ \pm}$, is possible and dominant (compare Figs. 5.1, 5.2 and 5.3). The decay via off-shell $W^{ \pm *}$ or $Z^{0 *}$ boson

$$
\tilde{\chi}_{1}^{ \pm} \rightarrow \tilde{\chi}_{1}^{0} W^{ \pm *} \rightarrow \tilde{\chi}_{1}^{0} l^{ \pm} \nu, \quad \tilde{\chi}_{2}^{0} \rightarrow \tilde{\chi}_{1}^{0} Z^{0 *} \rightarrow \tilde{\chi}_{1}^{0} l^{+} l^{-}
$$


is dominant for $m\left(\tilde{l}_{R}^{ \pm}\right) \gg m\left(\tilde{\chi}_{1}^{ \pm}\right) \approx m\left(\tilde{\chi}_{2}^{0}\right)$. In the intermediate region the decay channel via off-shell slepton has a non-negligible contribution.

The decay process has important effects on the transverse momentum of the leptons and the number of $\tau$ leptons in the event. In Figs. 5.2 and 5.3 it can be seen that the chargino dominantly decays into a stau slepton, if this decay is kinematically allowed and the decay into sneutrinos is kinematically not allowed. The reason is that the decay via on-shell particles is preferred over a decay via off-shell particles. The chargino is a mixture that has Higgsino and Wino components. For the lighter chargino the Wino component is dominant in the parameter regime considered. For the charged Wino no coupling to right-handed sleptons exists ${ }^{3}$. As the selectron and smuon have very low mixing between left- and righthanded form and the lighter stau has a significant left-handed component the charged Wino component of the lighter chargino prefers the decay into a stau. The Higgsino component has a Yukawa coupling to sleptons. In the approximation of Eqn. 2.20 the Higgsino component couples only to the stau. As both chargino components have a negligible coupling to the selectron and smuon, the chargino decay into selectron and smuon is negligible and in the region where the decay is kinematically allowed, the chargino decays mostly into a stau. For the next-to-lightest neutralino the Bino component couples to the selectron and the smuon and the decay is possible. However the decay into a stau slepton is enhanced as the Bino, Wino and Higgsino components of the neutralino couple to the stau.

At benchmark point BP1 the branching ratios of the major decay channels are given in Table 5.3. Benchmark point BP2 is in a region, where the decay via an on-shell slepton is kinematically forbidden. The decay of the chargino is via an off-shell $W^{ \pm *}$ boson and the decay channels with final states $\tilde{\chi}_{1}^{0} e \nu_{e}, \tilde{\chi}_{1}^{0} \mu \nu_{\mu}$ and $\tilde{\chi}_{1}^{0} \tau \nu_{\tau}$ have approximately the same branching ratio. The masses and branching ratios for the point BP2 can be found in the Tables 5.4 and 5.5. Obviously the preferred decay channel has an effect on the number of $\tau$ leptons in an event and for this reason also the number of events in the dilepton + track channel. The relevant quantities are the branching ratios of chargino and neutralino into three leptons with $i=0,1,2,3 \tau$ leptons, which can be seen in Fig. 5.4. In a region, where

\footnotetext{
${ }^{3}$ See Fig. 2.2 for the relevant couplings of the chargino and neutralino components.
} 
the decay via an on-shell stau slepton is kinematically allowed, almost all events have either one or three $\tau$ leptons as decay products of the chargino and the neutralino.

It can be seen in Fig. 5.1 that the masses of $\tilde{\chi}_{1}^{ \pm}$and $\tilde{\chi}_{2}^{0}$ have a small dependence on $m_{0}$. As a result the dependence of the cross section for their production on $m_{0}$ can be neglected. For Fig. 5.5 the cross section for the production of associated $\tilde{\chi}_{1}^{ \pm}$and $\tilde{\chi}_{2}^{0}$ in $p \bar{p}$ $\sqrt{s}=1.96 \mathrm{TeV}$ collisions at the Tevatron was calculated with Prospino $2.0[35]^{4}$. The low dependence on $m_{0}$ can be seen.
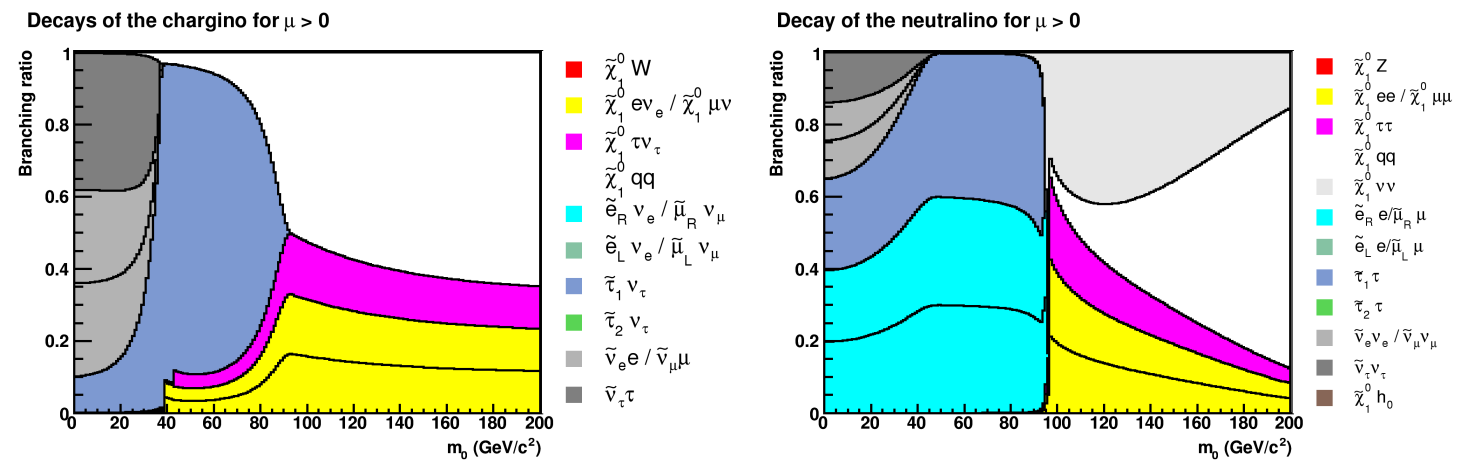

Figure 5.2: Branching ratios for the decay of chargino and neutralino as a function of $m_{0}$ at benchmark point BP1 with $\mu>0$. Benchmark point BP1 is at $m_{0}=60 \mathrm{GeV} / \mathrm{c}^{2}$; benchmark point BP2 is at $m_{0}=140 \mathrm{GeV} / \mathrm{c}^{2}$.
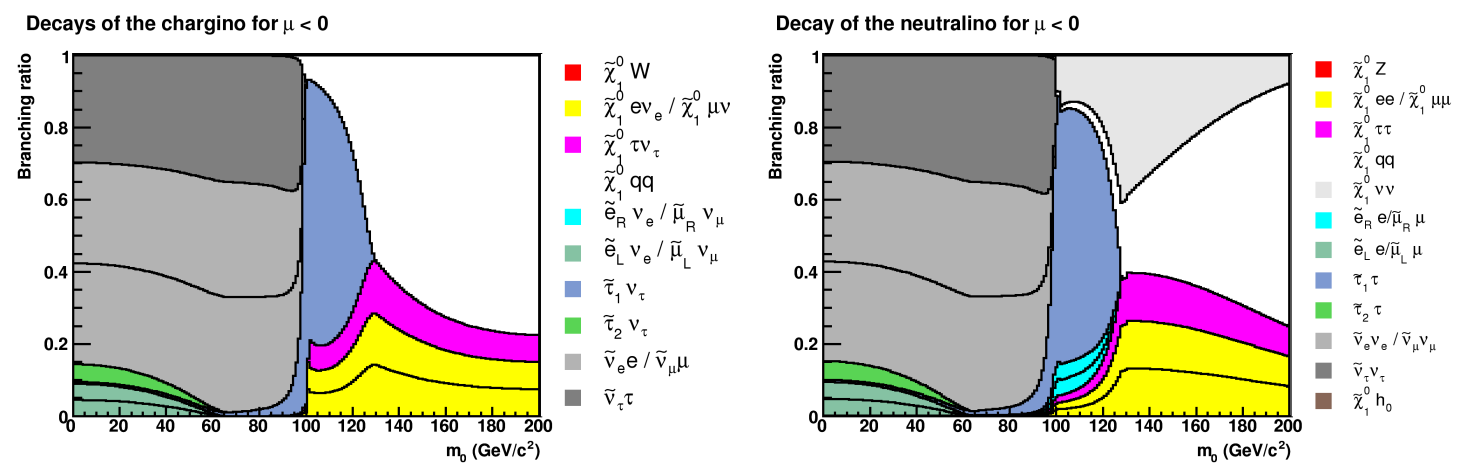

Figure 5.3: Branching ratios for the decay of chargino and neutralino as a function of $m_{0}$ at benchmark point BP3 with $\mu<0$. Benchmark point BP3 is at $m_{0}=110 \mathrm{GeV} / \mathrm{c}^{2}$.

\footnotetext{
${ }^{4}$ The particle spectrum used was produced with ISAJET $7.75[33]$.
} 


\begin{tabular}{|l|l|}
\hline Particle & Mass \\
\hline \hline$\tilde{\chi}_{2}^{0}$ & $124.4 \mathrm{GeV} / \mathrm{c}^{2}$ \\
\hline$\tilde{\chi}_{1}^{ \pm}$ & $121.9 \mathrm{GeV} / \mathrm{c}^{2}$ \\
\hline$\tilde{e}_{R}$ & $99.8 \mathrm{GeV} / \mathrm{c}^{2}$ \\
\hline$\tilde{\mu}_{R}$ & $99.8 \mathrm{GeV} / \mathrm{c}^{2}$ \\
\hline$\tilde{\tau}_{1}$ & $99.7 \mathrm{GeV} / \mathrm{c}^{2}$ \\
\hline$\tilde{\chi}_{1}^{0}$ & $67.8 \mathrm{GeV} / \mathrm{c}^{2}$ \\
\hline
\end{tabular}

Table 5.2: Mass of supersymmetric particles at benchmark point $\mathrm{BP} 1$ for $\mu>0$ (all other particles have higher masses).

\begin{tabular}{|l|l|}
\hline Particle & Mass \\
\hline \hline$\tilde{e}_{R}$ & $161.1 \mathrm{GeV} / \mathrm{c}^{2}$ \\
\hline$\tilde{\mu}_{R}$ & $161.1 \mathrm{GeV} / \mathrm{c}^{2}$ \\
\hline$\tilde{\tau}_{1}$ & $160.6 \mathrm{GeV} / \mathrm{c}^{2}$ \\
\hline$\tilde{\chi}_{2}^{0}$ & $125.7 \mathrm{GeV} / \mathrm{c}^{2}$ \\
\hline$\tilde{\chi}_{1}^{ \pm}$ & $123.4 \mathrm{GeV} / \mathrm{c}^{2}$ \\
\hline$\tilde{\chi}_{1}^{0}$ & $68.4 \mathrm{GeV} / \mathrm{c}^{2}$ \\
\hline
\end{tabular}

Table 5.4: Mass of supersymmetric particles at benchmark point BP2 (all other particles have higher masses).

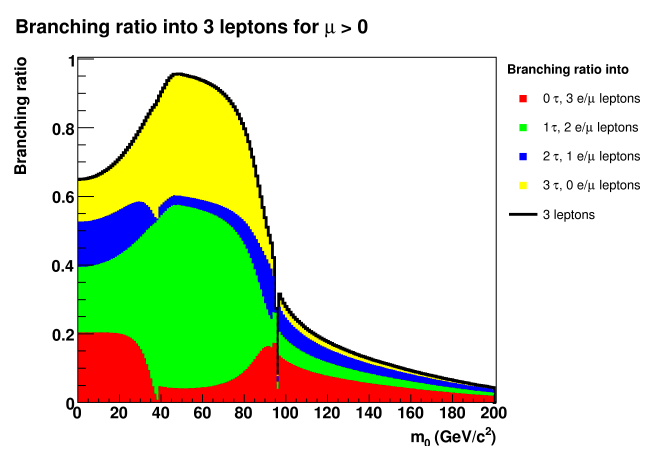

\begin{tabular}{|c|l|}
\hline$\tilde{\chi}_{1}^{+} \rightarrow$ & $\mathrm{BR}$ \\
\hline \hline$\tilde{\tau}_{1}^{+} \nu_{\tau}$ & $82.6 \%$ \\
\hline$\tilde{\chi}_{1}^{0} \tau^{+} \nu_{\tau}$ & $4.0 \%$ \\
\hline$\tilde{\chi}_{1}^{0} e^{+} \nu_{e}$ & $3.7 \%$ \\
\hline$\tilde{\chi}_{1}^{0} \mu^{+} \nu_{\mu}$ & $3.7 \%$ \\
\hline$\tilde{\chi}_{1}^{0} \bar{d} u$ & $3.0 \%$ \\
\hline$\tilde{\chi}_{1}^{0} \bar{s} c$ & $3.0 \%$ \\
\hline
\end{tabular}

Table 5.3: Branching ratios for the different decay channels of the chargino at benchmark point BP1 for $\mu>0$. Decay channels with branching ratio smaller than $1 \%$ are neglected.

\begin{tabular}{|c|l|}
\hline$\tilde{\chi}_{1}^{+} \rightarrow$ & $\mathrm{BR}$ \\
\hline \hline$\tilde{\chi}_{1}^{0} \bar{d} u$ & $30.3 \%$ \\
\hline$\tilde{\chi}_{1}^{0} \bar{s} c$ & $30.3 \%$ \\
\hline$\tilde{\chi}_{1}^{0} \tau^{+} \nu_{\tau}$ & $13.2 \%$ \\
\hline$\tilde{\chi}_{1}^{0} e^{+} \nu_{e}$ & $13.1 \%$ \\
\hline$\tilde{\chi}_{1}^{0} \mu^{+} \nu_{\mu}$ & $13.1 \%$ \\
\hline$\tilde{\tau}_{1}^{+} \nu_{\tau}$ & $0.0 \%$ \\
\hline
\end{tabular}

Table 5.5: Branching ratios for the different decay channels of the chargino at benchmark point BP2. Decay channels with branching ratio smaller than $1 \%$ are neglected.

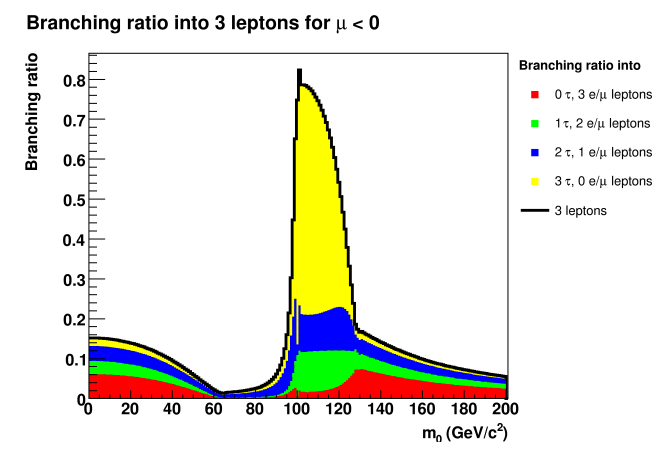

Figure 5.4: Branching ratio of $\tilde{\chi}_{1}^{ \pm} \tilde{\chi}_{2}^{0}$ into three leptons split into final states with $0,1,2$ or $3 \tau$ leptons as a function of $m_{1 / 2}$ at benchmark point BP1 with $\mu>0$ (left) and at benchmark point BP3 with $\mu<0$ (right). Benchmark point BP1 is at $m_{0}=60 \mathrm{GeV} / \mathrm{c}^{2}$ for $\mu>0$, benchmark point BP2 is at $m_{0}=140 \mathrm{GeV} / \mathrm{c}^{2}$ for $\mu>0$ and benchmark point BP3 is at $m_{0}=110 \mathrm{GeV} / \mathrm{c}^{2}$ for $\mu<0$. 

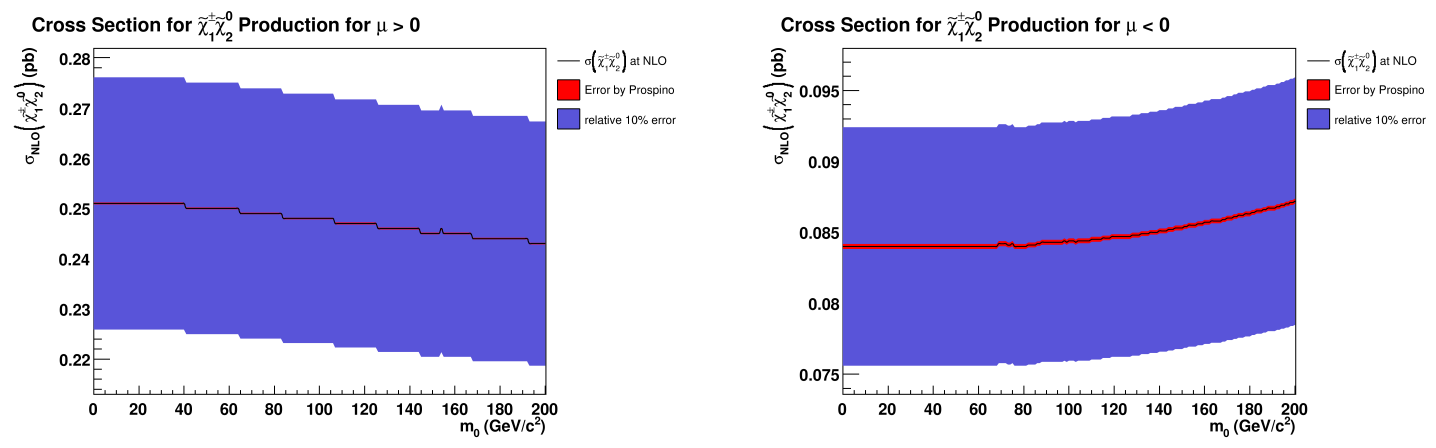

Figure 5.5: Cross section for the production of $\tilde{\chi}_{1}^{ \pm} \tilde{\chi}_{2}^{0}$ in $p \bar{p}$ collisions at the Tevatron as a function of $m_{0}$ at benchmark point BP1 with $\mu>0$ (left) and at benchmark point BP3 with $\mu<0$ (right). Benchmark point $\mathrm{BP} 1$ is at $m_{0}=60 \mathrm{GeV} / \mathrm{c}^{2}$ for $\mu>0$, benchmark point $\mathrm{BP} 2$ is at $m_{0}=140 \mathrm{GeV} / \mathrm{c}^{2}$ for $\mu>0$ and benchmark point BP3 is at $m_{0}=110 \mathrm{GeV} / \mathrm{c}^{2}$ for $\mu<0$. 


\subsubsection{The Common Gaugino Mass $m_{1 / 2}$}

The mSUGRA parameter $m_{1 / 2}$ is important for the masses of the relevant particles (and thus the cross section for their production); all relevant particle masses have a strong dependence on $m_{1 / 2}$. However, Fig. 5.6 shows, that sleptons and charginos or neutralinos show a
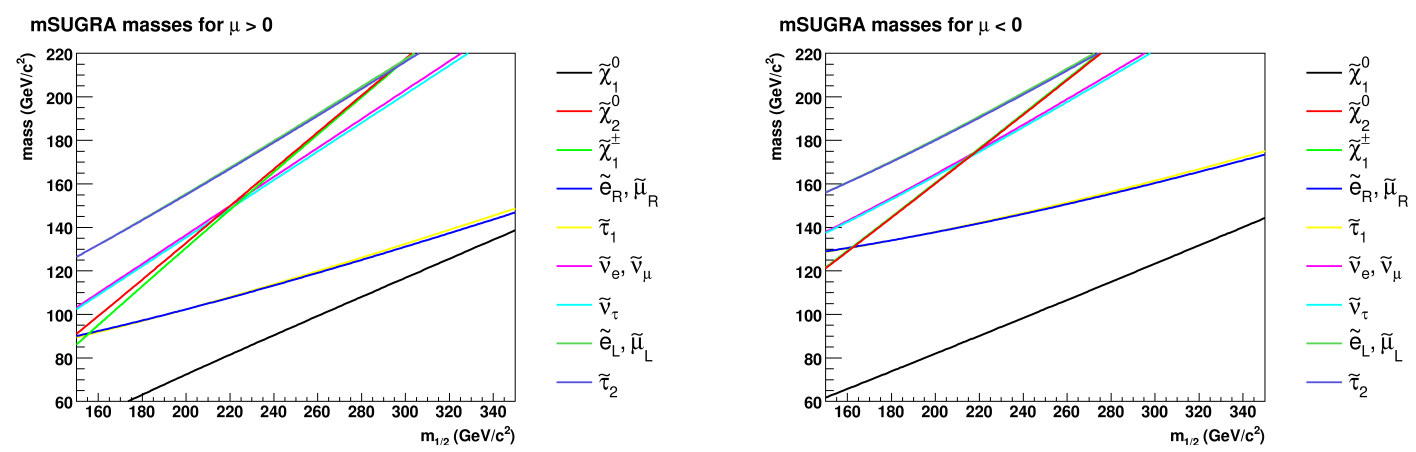

Figure 5.6: Mass of the relevant supersymmetric particles as a function of $m_{1 / 2}$ at benchmark point BP1 with $\mu>0$ (left) and at benchmark point BP3 with $\mu<0$ (right). The benchmark points BP1 and BP3 are at $m_{1 / 2}=190 \mathrm{GeV} / \mathrm{c}^{2}$ for $\mu>0$ and $\mu<0$ respectively.

different dependence on $m_{1 / 2}$ and for rising values of $m_{1 / 2}$ the following decay channels are possible:

1. The decay via off-shell particles is always possible, but it is suppressed as long as decay channels via on-shell particles are open. The decay via off-shell particles is mostly a decay via off-shell $W^{ \pm *}$ or $Z^{0 *}$ boson, where quarks dominate the final state, whereas in the decay via off-shell sleptons leptons and neutrinos are dominant in the final state. In the region, where $m\left(\tilde{l}_{R}^{ \pm}\right) \approx m\left(\tilde{\chi}_{2}^{0}\right)$ the decay via off-shell sleptons is dominant for a small $m_{1 / 2}$ range.

$$
\begin{gathered}
\tilde{\chi}_{1}^{ \pm} \rightarrow \tilde{\chi}_{1}^{0} W^{ \pm *} \rightarrow\left\{\begin{array}{l}
\tilde{\chi}_{1}^{0} l^{ \pm} \nu_{l} \\
\tilde{\chi}_{1}^{0} q_{1} \bar{q}_{2}
\end{array} \quad, \quad \tilde{\chi}_{2}^{0} \rightarrow \tilde{\chi}_{1}^{0} Z^{0 *} \rightarrow\left\{\begin{array}{c}
\tilde{\chi}_{1}^{0} l^{+} l^{-} \\
\tilde{\chi}_{1}^{0} q \bar{q} \\
\tilde{\chi}_{1}^{0} \nu \bar{\nu}
\end{array}\right.\right. \\
\tilde{\chi}_{1}^{ \pm} \rightarrow \tilde{l}_{R}^{ \pm *} \nu_{l} \rightarrow \tilde{\chi}_{2}^{0} l^{ \pm} \nu_{l} \quad, \quad \tilde{\chi}_{2}^{0} \rightarrow \tilde{l}_{R}^{ \pm *} l^{\mp} \rightarrow \tilde{\chi}_{2}^{0} l^{+} l^{-}
\end{gathered}
$$


2. If the slepton mass is lower than the mass of the chargino and neutralino, the decay via an on-shell right-handed slepton is possible and, as long as no other decay via on-shell particles is allowed, this decay is dominant.

$$
\tilde{\chi}_{1}^{ \pm} \rightarrow \tilde{l}_{R}^{ \pm} \nu_{l} \quad, \quad \tilde{\chi}_{2}^{0} \rightarrow \tilde{l}_{R}^{ \pm} l^{\mp}
$$

3. If the sneutrino mass is lower than the mass of the chargino and the neutralino, the decay

$$
\tilde{\chi}_{1}^{ \pm} \rightarrow \tilde{\nu}_{l} l^{ \pm} \quad, \quad \tilde{\chi}_{2}^{0} \rightarrow \tilde{\nu}_{l} \nu_{l}
$$

is possible. In the decay of the neutralino via an off-shell sneutrino finally an LSP and two neutrinos are in the final state. As neutrinos are visible only via missing transverse energy in the detector, it is hard to see such a decay. The trilepton analysis is not sensitive to this decay.

4. If the mass of the left-handed sleptons is lower than the mass of the chargino and neutralino, the decay

$$
\tilde{\chi}_{1}^{ \pm} \rightarrow \tilde{l}_{L}^{ \pm} \nu_{l} \quad, \quad \tilde{\chi}_{2}^{0} \rightarrow \tilde{l}_{L}^{ \pm} l^{\mp}
$$

is possible.

5. If $m\left(\tilde{\chi}_{1}^{ \pm}\right) \geq m\left(\tilde{\chi}_{1}^{0}\right)+m\left(W^{ \pm}\right), m\left(\tilde{\chi}_{2}^{0}\right) \geq m\left(\tilde{\chi}_{1}^{0}\right)+m\left(Z^{0}\right)$ or $m\left(\tilde{\chi}_{2}^{0}\right) \geq m\left(\tilde{\chi}_{1}^{0}\right)+$ $m\left(h_{0}\right)$, the decay channels

$$
\tilde{\chi}_{1}^{ \pm} \rightarrow \tilde{\chi}_{1}^{0} W^{ \pm} \quad, \quad \tilde{\chi}_{2}^{0} \rightarrow \tilde{\chi}_{1}^{0} Z^{0}, \quad \tilde{\chi}_{2}^{0} \rightarrow \tilde{\chi}_{1}^{0} h_{0}
$$

are allowed. Figure 2.2 shows, that the chargino can decay into a $W^{ \pm}$boson and the lightest neutralino via the Wino-Wino-W vertex and the Higgsino-Higgsino-W vertex, while the neutralino can decay into the $Z^{0}$ boson and the lightest neutralino only via the Higgsino-Higgsino-Z vertex. As $\tilde{\chi}_{1}^{ \pm}$and $\tilde{\chi}_{2}^{0}$ have a strong Wino component the decay of the $\tilde{\chi}_{2}^{0}$ into a $Z^{0}$ is suppressed, while the decay of the $\tilde{\chi}_{1}^{ \pm}$into a $W^{ \pm}$may be significant based on the chosen set of parameters.

All other decays are negligible in the considered parameter space. In Figs. 5.7 and 5.8 the branching ratios for the different decay channels can be seen. 

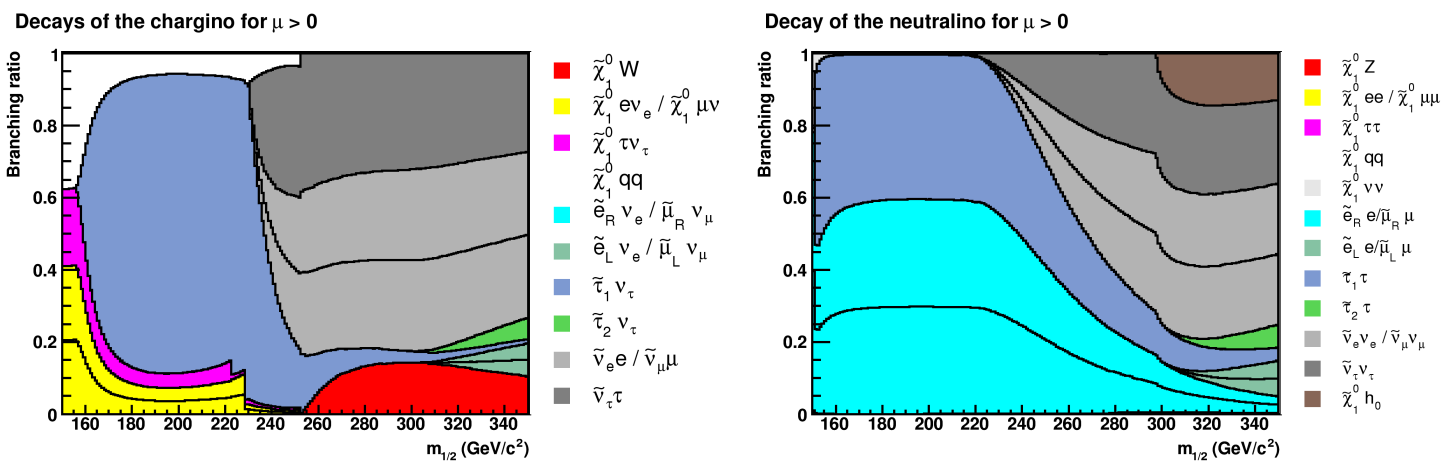

Figure 5.7: Branching ratios for the decay of chargino and neutralino as a function of $m_{1 / 2}$ at benchmark point BP1 with $\mu>0$. Benchmark point BP1 is at $m_{1 / 2}=190 \mathrm{GeV} / \mathrm{c}^{2}$.
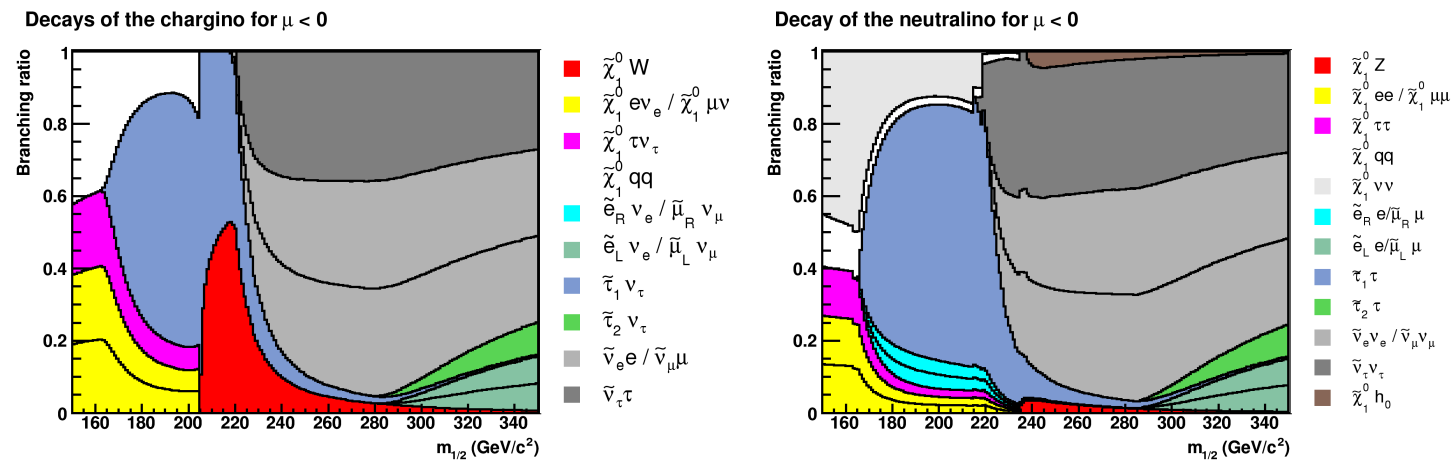

Figure 5.8: Branching ratios for the decay of chargino and neutralino as a function of $m_{1 / 2}$ at benchmark point BP3 with $\mu<0$. Benchmark point BP3 is at $m_{1 / 2}=190 \mathrm{GeV} / \mathrm{c}^{2}$.
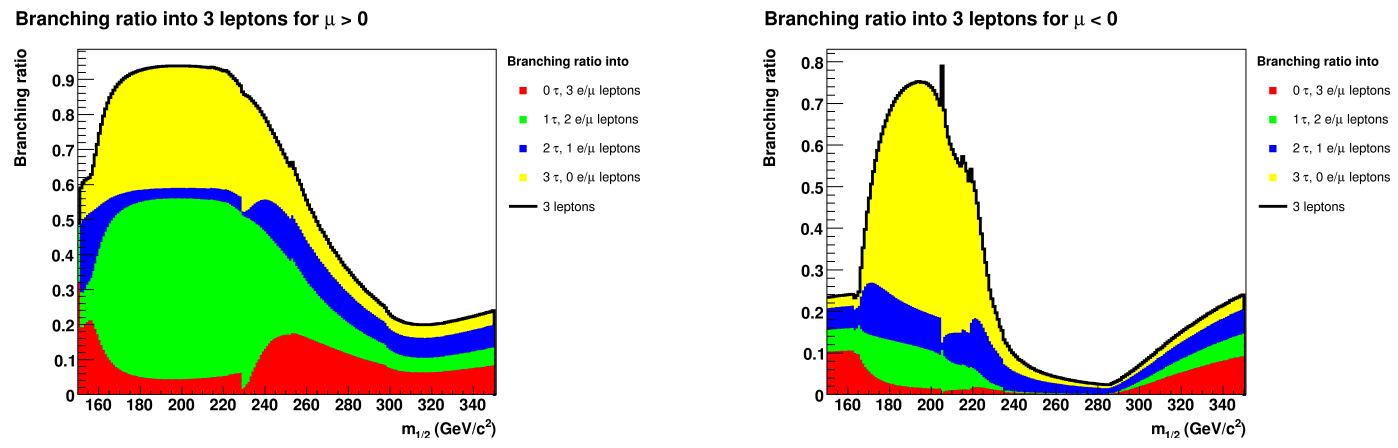

Figure 5.9: Branching ratio into three leptons split into final states with 0,1,2 or $3 \tau$ leptons as a function of $m_{1 / 2}$ at benchmark point BP1 with $\mu>0$ (left) and at benchmark point BP3 with $\mu<0$ (right). The benchmark points BP1 and BP3 are at $m_{1 / 2}=190 \mathrm{GeV} / \mathrm{c}^{2}$ for $\mu>0$ and $\mu<0$ respectively. 

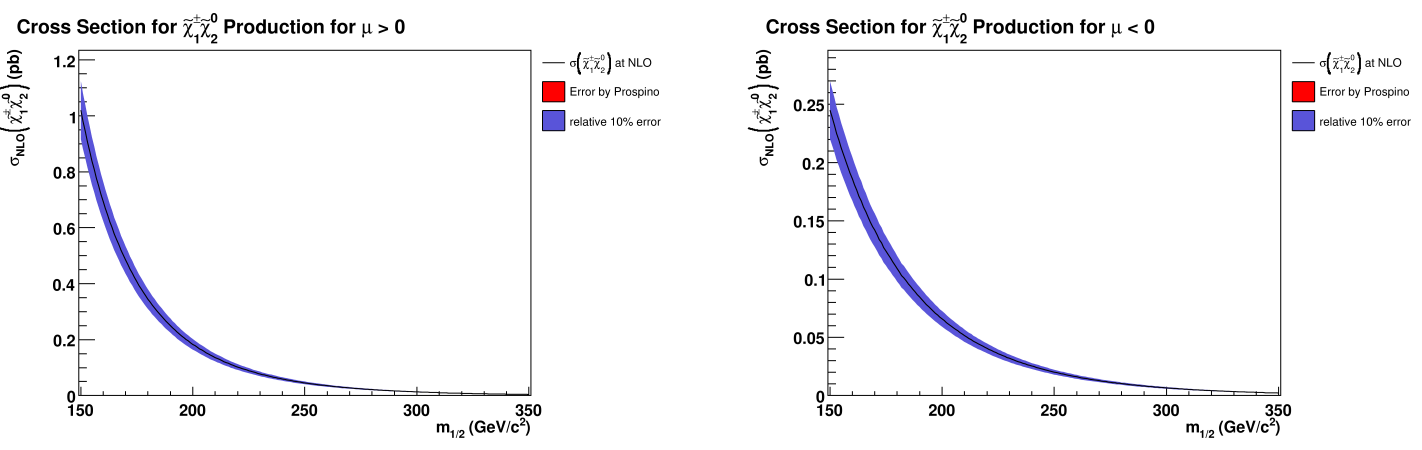

Figure 5.10: Cross section for the production of $\tilde{\chi}_{1}^{ \pm} \tilde{\chi}_{2}^{0}$ in $p \bar{p}$ collisions at the Tevatron as a function of $m_{1 / 2}$ at benchmark point BP1 with $\mu>0$ (left) and at benchmark point BP3 with $\mu<0$ (right). The benchmark points BP1 and BP3 are at $m_{1 / 2}=190 \mathrm{GeV} / \mathrm{c}^{2}$ for $\mu>0$ and $\mu<0$ respectively. 


\subsubsection{The Ratio of the Vacuum Expectation Values of the two Higgs Doublets $\tan \beta$}

The value of $\tan \beta$ has an influence on the mixing of the third generation sleptons. With increasing $\tan \beta$ the mixing of the $\tilde{\tau}$ sleptons increases and thus also the difference in mass between the selectron or smuon and the stau slepton. The masses of all other particles show less dependence on $\tan \beta$.

In Figs. 5.12 and 5.13 the branching ratios for the relevant decay channels are shown. For $\tan \beta<1.5$ perturbation theory breaks down as one of the Yukawa couplings is greater than 10. For $\mu>0$ and $\tan \beta=15.3$ ISAJET can not find a solution for the renormalization group equation.

It can be seen that with increasing $\tan \beta$ the decay into third generation sleptons is favored and for this reason more $\tau$ leptons will be in the final state. This is especially visible for the decay of the neutralino which does not favor the third generation sleptons as much as the decay of the chargino does. The reason for this is, that the $\tilde{\tau}_{1}$ slepton is a mixture of $\tilde{\tau}_{L}$ and $\tilde{\tau}_{R}$. For increasing $\tan \beta$ the $\tilde{\tau}_{1}$ becomes more $\tilde{\tau}_{L}$-like. As the lighter chargino and the next-to-lightest neutralino are mostly Winos, which couple to left-handed sleptons only, the decay into $\tilde{\tau}_{1}$ becomes more favored. Figure 5.14 shows that the branching ratio into final states with two electrons or muons and one $\tau$ lepton decreases for increasing $\tan \beta$ as the branching ratio of the decay $\tilde{\chi}_{2}^{0} \rightarrow \tilde{\tau}_{1}^{ \pm} \tau^{\mp} \rightarrow \tilde{\chi}_{1}^{0} \tau^{+} \tau^{-}$increases. The dependence of the cross section on $\tan \beta$ follows the mass dependence of $\tilde{\chi}_{1}^{ \pm}$and $\tilde{\chi}_{2}^{0}$.
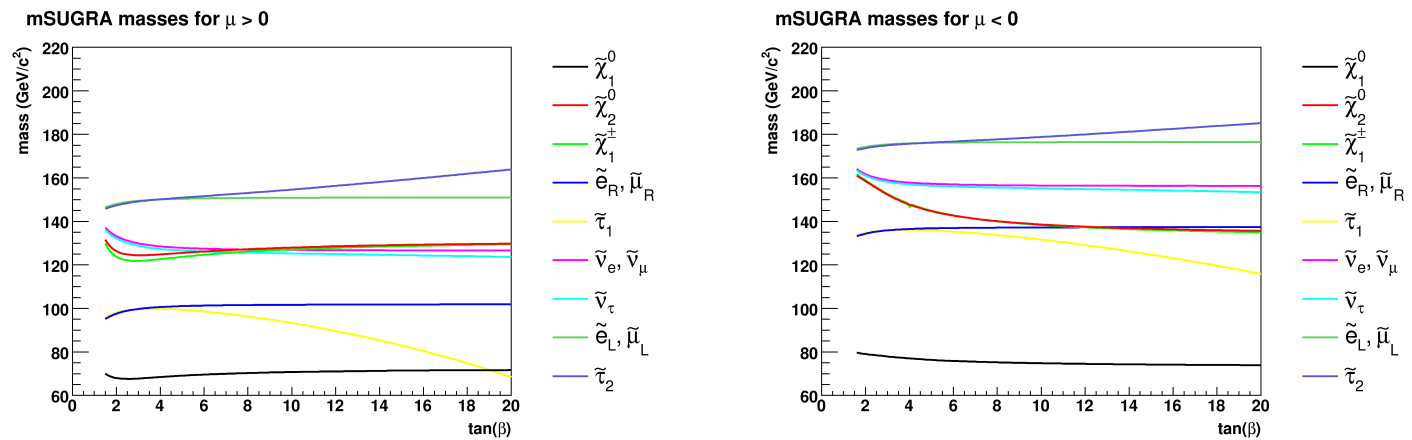

Figure 5.11: Mass of the relevant supersymmetric particles as a function of $m_{1 / 2}$ at benchmark point BP1 with $\mu>0$ (left) and at benchmark point BP3 with $\mu<0$ (right). The benchmark points BP1 and BP3 are at $\tan \beta=3$ for $\mu>0$ and $\mu<0$ respectively. 

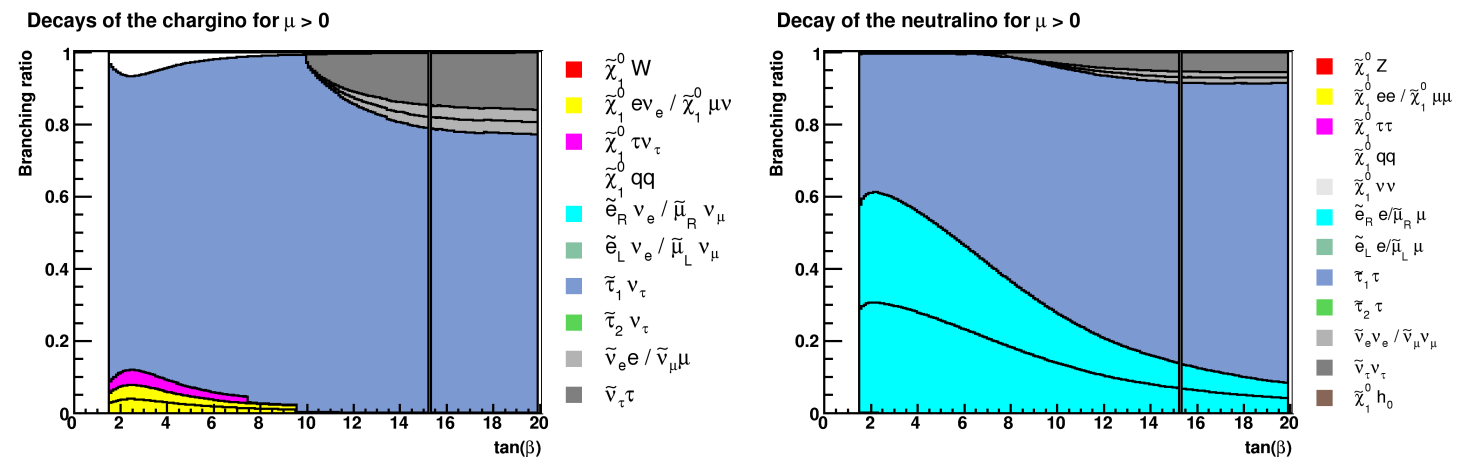

Figure 5.12: Branching ratios for the decay of chargino and neutralino as a function of $\tan \beta$ at benchmark point BP1 with $\mu>0$. Benchmark point BP1 is at $\tan \beta=3$.
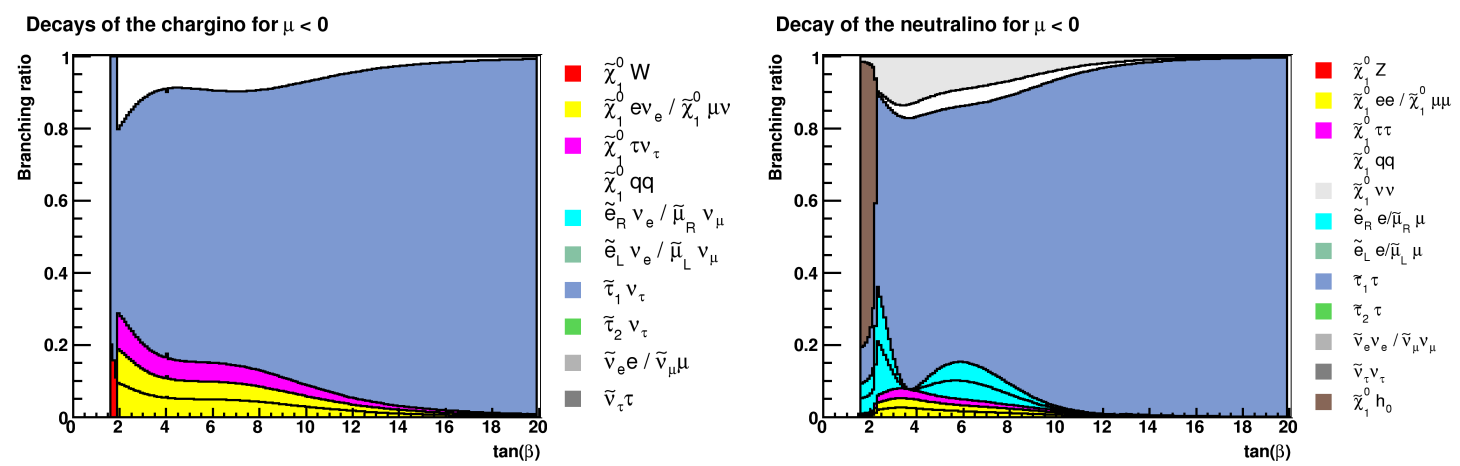

Figure 5.13: Branching ratios for the decay of chargino and neutralino as a function of $\tan \beta$ at benchmark point BP3 with $\mu<0$. Benchmark point BP3 is at $\tan \beta=3$.
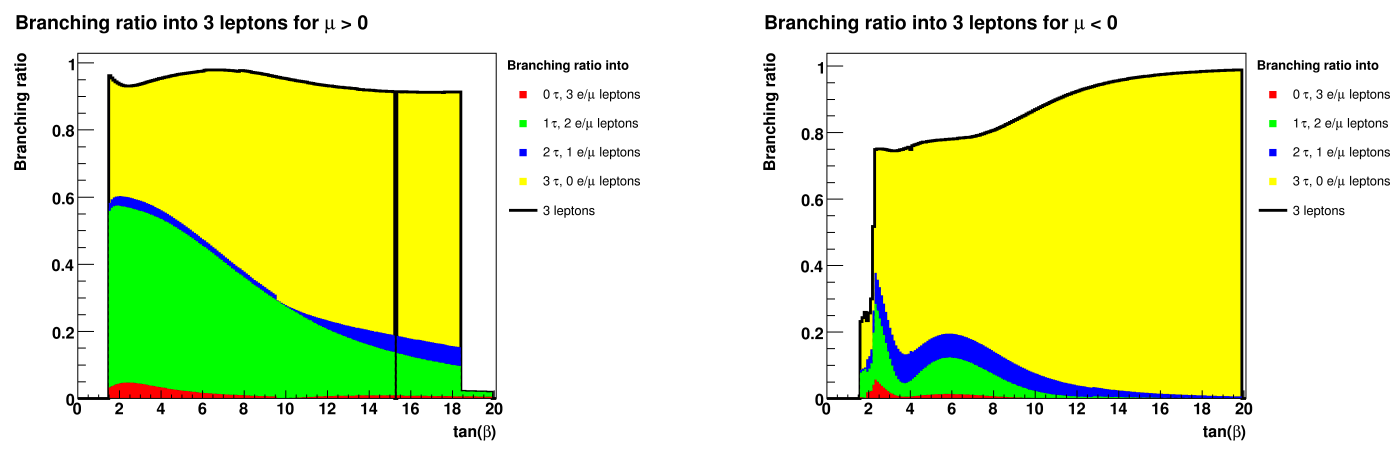

Figure 5.14: Branching ratio into three leptons split into final states with $0,1,2$ or $3 \tau$ leptons as a function of $\tan \beta$ at benchmark point BP1 with $\mu>0$ (left) and at benchmark point BP3 with $\mu<0$ (right). The benchmark points BP1 and BP3 are at $\tan \beta$ for $\mu>0$ and $\mu<0$ respectively. 

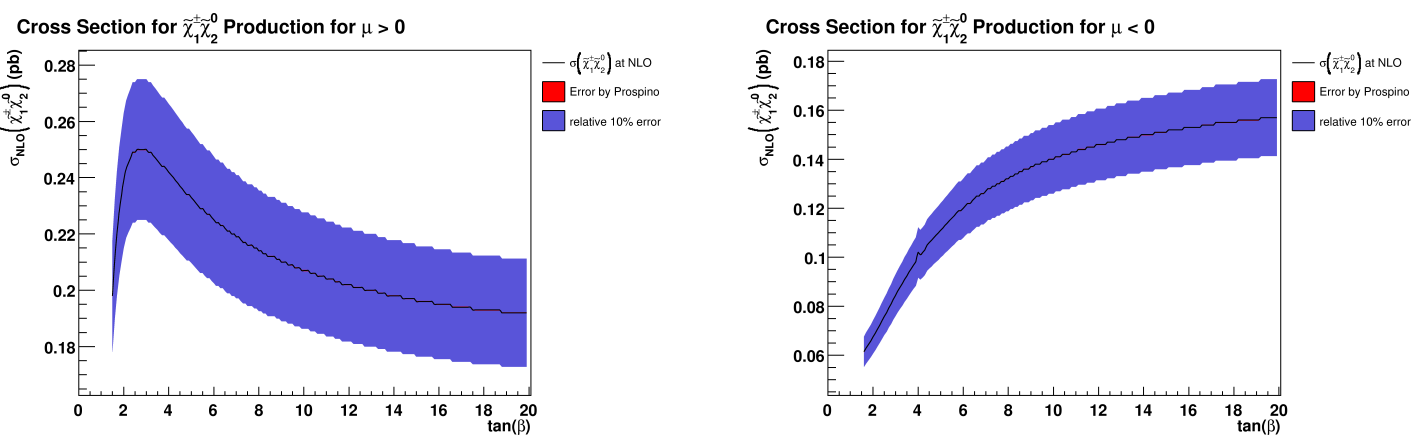

Figure 5.15: Cross section for the production of $\tilde{\chi}_{1}^{ \pm} \tilde{\chi}_{2}^{0}$ in $p \bar{p}$ collisions at the Tevatron as a function of $\tan \beta$ at benchmark point BP1 with $\mu>0$ (left) and at benchmark point BP3 with $\mu<0$ (right). The benchmark points BP1 and BP3 are at $\tan \beta=3$ for $\mu>0$ and $\mu<0$ respectively. 


\subsubsection{The Common Trilinear Coupling $A_{0}$}

The neutralino and chargino masses show a low dependence on $A_{0}$, while there is only a very low mass dependence visible for all other supersymmetric particles. Fig. 5.18 for $\mu<0$ shows that this mass dependence has no major effects on the branching ratios of the different decay channels provided no other decay channels open up. In Fig. 5.17 for $\mu>0$ the decay channel into on-shell sleptons is open for small trilinear coupling and closed for bigger trilinear couplings. This has an effect on the branching ratios. In general the dependence of the branching ratios on $A_{0}$ is rather weak.
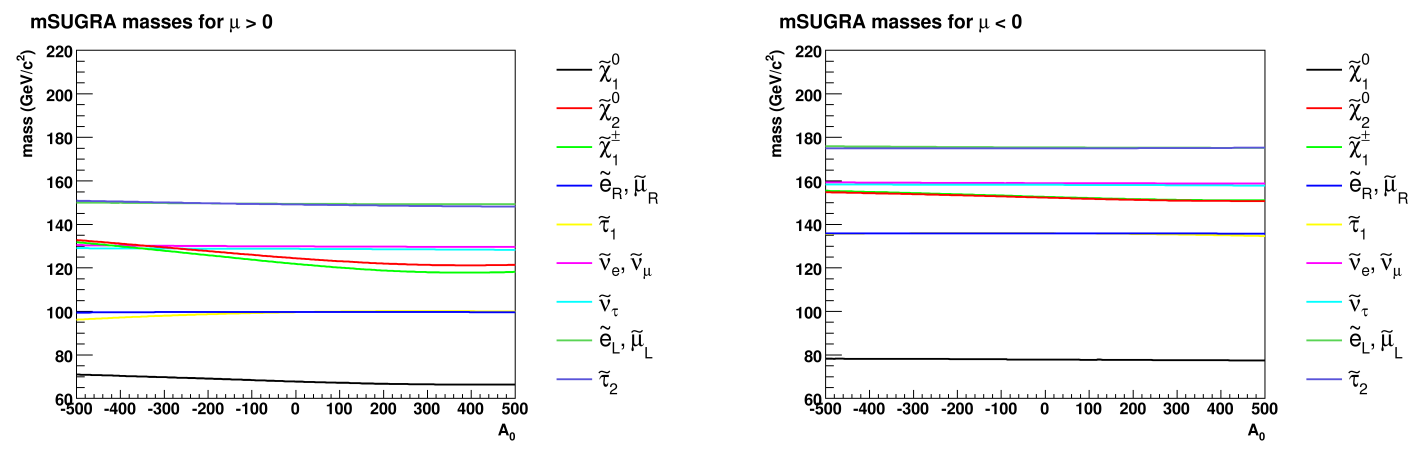

Figure 5.16: Mass of the relevant supersymmetric particles as a function of $A_{0}$ at benchmark point BP1 with $\mu>0$ (left) and at benchmark point BP3 with $\mu<0$ (right). The benchmark points $\mathrm{BP} 1$ and $\mathrm{BP} 3$ are at $A_{0}=0 \mathrm{GeV}$ for $\mu>0$ and $\mu<0$ respectively. 

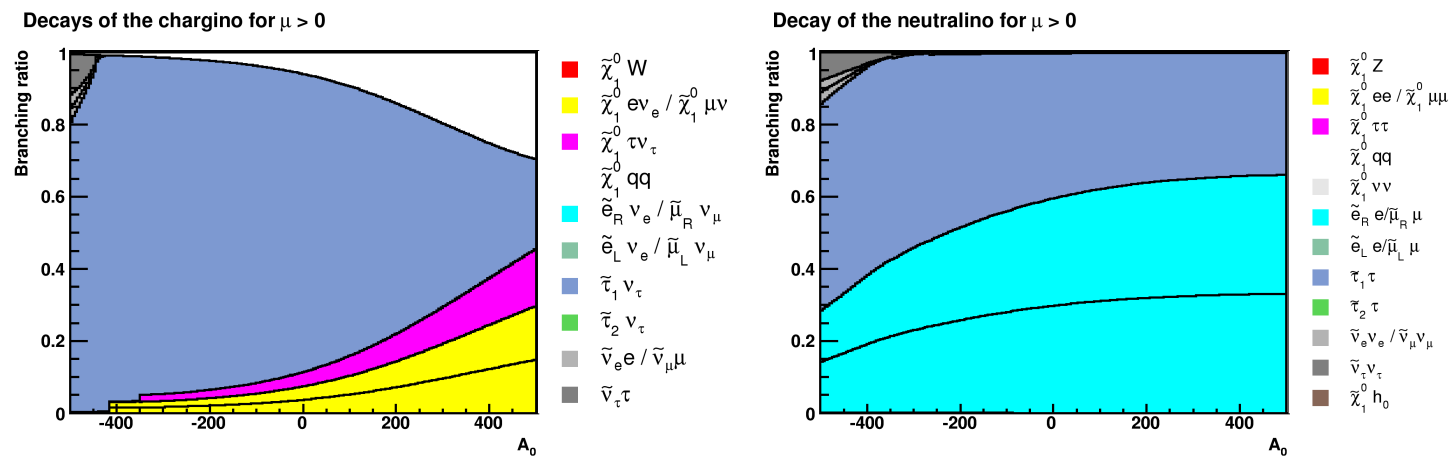

Figure 5.17: Branching ratios for the decay of chargino and neutralino as a function of $A_{0}$ at benchmark point $\mathrm{BP} 1$ with $\mu>0$. Benchmark point $\mathrm{BP} 1$ is at $A_{0}=0 \mathrm{GeV}$.
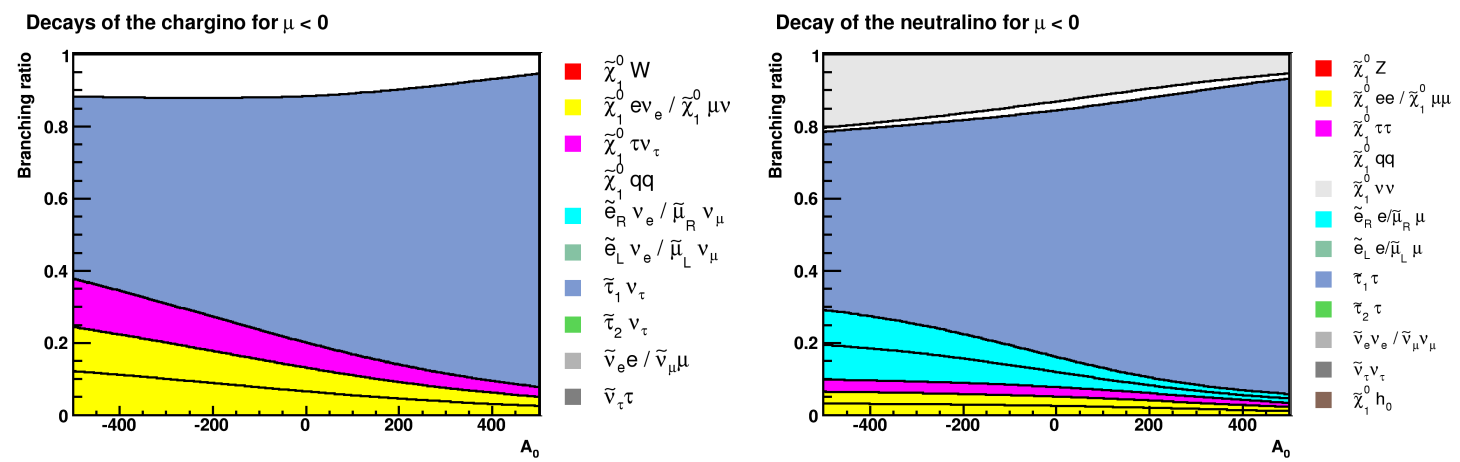

Figure 5.18: Branching ratios for the decay of chargino and neutralino as a function of $A_{0}$ at benchmark point BP3 with $\mu<0$. Benchmark point BP3 is at $A_{0}=0 \mathrm{GeV}$.
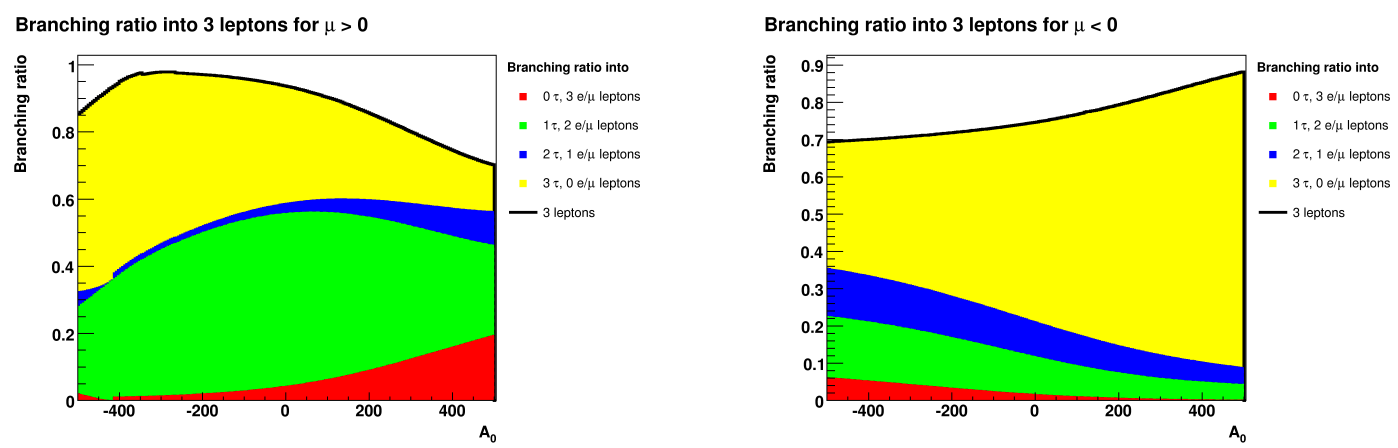

Figure 5.19: Branching ratio into three leptons split into final states with $0,1,2$ or $3 \tau$ leptons as a function of $A_{0}$ at benchmark point BP1 with $\mu>0$ (left) and at benchmark point BP3 with $\mu<0$ (right). The benchmark points BP1 and BP3 are at $A_{0}=0 \mathrm{GeV}$ for $\mu>0$ and $\mu<0$ respectively. 

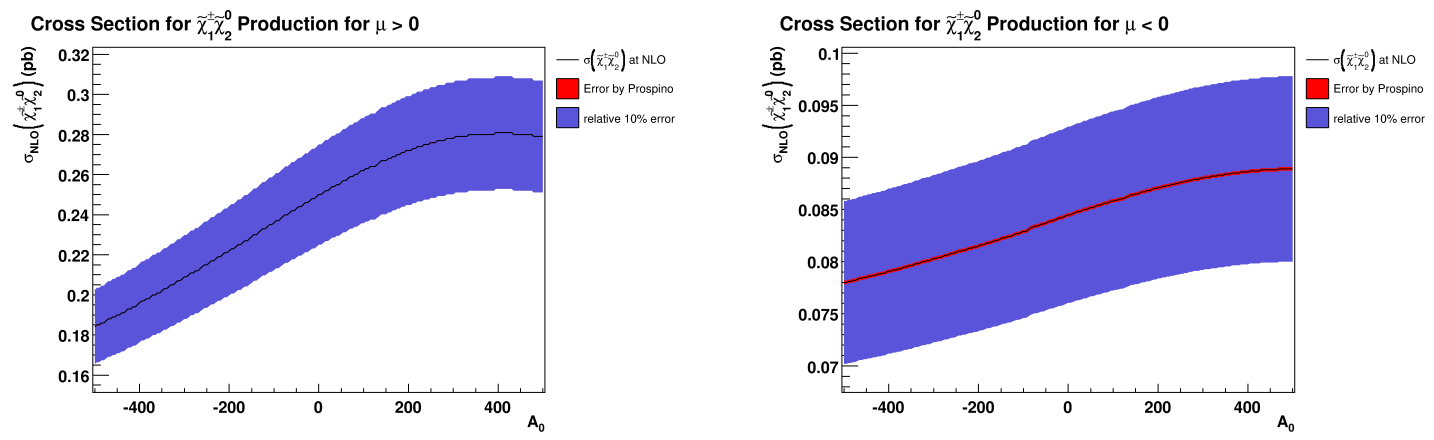

Figure 5.20: Cross section for the production of $\tilde{\chi}_{1}^{ \pm} \tilde{\chi}_{2}^{0}$ in $p \bar{p}$ collisions at the Tevatron as a function of $A_{0}$ at benchmark point BP1 with $\mu>0$ (left) and at benchmark point BP3 with $\mu<0$ (right). The benchmark points BP1 and BP3 are at $A_{0}=0 \mathrm{GeV}$ for $\mu>0$ and $\mu<0$ respectively. 


\subsection{Description of the Sensitivity of the Analysis}

In the following we limit the discussion to the mSUGRA parameters $m_{0}$ and $m_{1 / 2}$ which we have identified as the parameters that have the biggest influence on the sensitivity of the analysis. The following parameters are fixed:

- $\tan \beta=3$

- $A_{0}=0 \mathrm{GeV}$

- $\mu>0$

\subsubsection{Regions in mSUGRA Parameter Space}

It was shown in section 5.1, that, based on the allowed decay channels, the mSUGRA parameter space can be divided into different phenomenology classes. Figure 5.21 divides the $\left(m_{0}, m_{1 / 2}\right)$-parameter space into 3 regions:

\section{Regions in the mSUGRA parameter space}

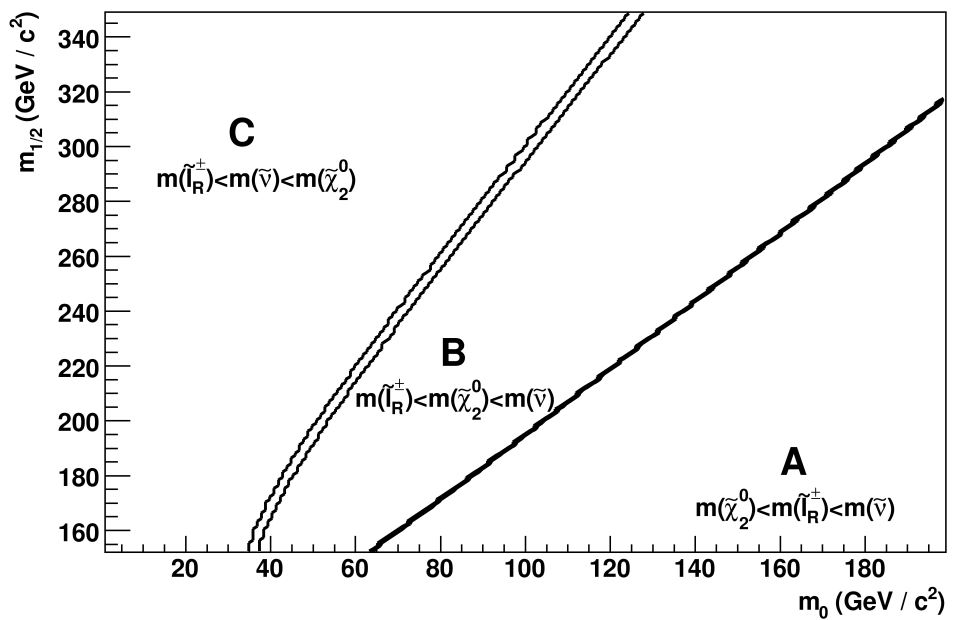

Figure 5.21: Based on the different dependences of slepton, chargino and neutralino masses on the mSUGRA parameters, regions with different possible decay channels can be defined in the mSUGRA parameter space. 


\section{Region A: Only three-body decays via off-shell particles}

Region A is defined by the mass relations

$$
\begin{gathered}
m\left(\tilde{\chi}_{2}^{0}\right)<m\left(\tilde{l}_{R}^{ \pm}\right)<m(\tilde{\nu})<m\left(\tilde{l}_{L}^{ \pm}\right) \\
m\left(\tilde{\chi}_{1}^{ \pm}\right)<m\left(\tilde{l}_{R}^{ \pm}\right)<m(\tilde{\nu})<m\left(\tilde{l}_{L}^{ \pm}\right) \\
m\left(\tilde{\chi}_{2}^{0}\right)<\left\{\begin{array}{l}
m\left(\tilde{\chi}_{1}^{0}\right)+m\left(Z^{0}\right) \quad ; m\left(\tilde{\chi}_{1}^{ \pm}\right)<m\left(\tilde{\chi}_{1}^{0}\right)+m\left(W^{ \pm}\right) \\
m\left(\tilde{\chi}_{1}^{0}\right)+m\left(h^{0}\right)
\end{array}\right.
\end{gathered}
$$

where $\tilde{l}_{R}^{ \pm}=\tilde{e}_{R}^{ \pm}, \tilde{\mu}_{R}^{ \pm}, \tilde{\tau}_{1}^{ \pm}$and $\tilde{l}_{L}^{ \pm}=\tilde{e}_{L}^{ \pm}, \tilde{\mu}_{L}^{ \pm}, \tilde{\tau}_{2}^{ \pm}$. As in mSUGRA $m\left(\tilde{\chi}_{1}^{ \pm}\right) \approx m\left(\tilde{\chi}_{2}^{0}\right)$ the relations 5.9 and 5.10 are equivalent ${ }^{5}$. In the parameter space considered relation 5.11 holds for $m_{1 / 2}<250 \mathrm{GeV} / \mathrm{c}^{2}$.

In region A only three-body decays via off-shell particles are possible and the relevant decays are

- via off-shell $W^{ \pm *}$ or $Z^{0 *}$ boson

$$
\tilde{\chi}_{2}^{0} \rightarrow \tilde{\chi}_{1}^{0} Z^{0 *} \rightarrow \tilde{\chi}_{1}^{0}\left\{\begin{array}{l}
l^{+} l^{-} \\
q \bar{q} \\
\nu \bar{\nu}
\end{array} \quad ; \quad \tilde{\chi}_{1}^{ \pm} \rightarrow \tilde{\chi}_{1}^{0} W^{ \pm *} \rightarrow \tilde{\chi}_{1}^{0}\left\{\begin{array}{l}
{ }^{ \pm} \nu_{l} \\
q_{1} \bar{q}_{2}
\end{array}\right.\right.
$$

- via off-shell right-handed slepton $\tilde{e}_{R}, \tilde{\mu}_{R}$ or $\tilde{\tau}_{1}$

$$
\tilde{\chi}_{2}^{0} \rightarrow \tilde{l}_{R}^{ \pm *} l^{\mp} \rightarrow \tilde{\chi}_{1}^{0} l^{+} l^{-} ; \quad \tilde{\chi}_{1}^{ \pm} \rightarrow \tilde{l}_{R}^{ \pm *} \nu_{l} \rightarrow \tilde{\chi}_{1}^{0} l^{ \pm} \nu_{l}
$$

- via off-shell sneutrino $\tilde{\nu}_{e}, \tilde{\nu}_{\mu}$ or $\tilde{\nu}_{\tau}$

$$
\tilde{\chi}_{2}^{0} \rightarrow \tilde{\nu}_{l}^{*} \bar{\nu}_{l} \rightarrow \tilde{\chi}_{1}^{0} \nu_{l} \bar{\nu}_{l} ; \quad \tilde{\chi}_{1}^{ \pm} \rightarrow \tilde{\nu}_{l}^{*} l^{ \pm} \rightarrow \tilde{\chi}_{1}^{0} l^{ \pm} \nu_{l}
$$

The decay via off-shell $W^{ \pm *}$ or $Z^{0 *}$ boson is dominant for higher $m_{0}$. At the border of region $\mathrm{A}$ it is $m\left(\tilde{\chi}_{2}^{0}\right) \approx m\left(\tilde{l}_{R}^{ \pm}\right)$and the decay via off-shell slepton is dominant.

\footnotetext{
${ }^{5}$ See section 2.2 .5 for details.
} 


\section{Region B: Decay via on-shell right-handed sleptons dominant}

Region $\mathrm{B}$ is defined by the mass relations

$$
\begin{array}{r}
m\left(\tilde{l}_{R}^{ \pm}\right)<m\left(\tilde{\chi}_{2}^{0}\right)<m(\tilde{\nu})<m\left(\tilde{l}_{L}^{ \pm}\right) \\
m\left(\tilde{l}_{R}^{ \pm}\right)<m\left(\tilde{\chi}_{1}^{ \pm}\right)<m(\tilde{\nu})<m\left(\tilde{l}_{L}^{ \pm}\right) \\
m\left(\tilde{\chi}_{2}^{0}\right)<\left\{\begin{array}{l}
m\left(\tilde{\chi}_{1}^{0}\right)+m\left(Z^{0}\right) \quad ; m\left(\tilde{\chi}_{1}^{ \pm}\right)<m\left(\tilde{\chi}_{1}^{0}\right)+m\left(W^{ \pm}\right) \\
m\left(\tilde{\chi}_{1}^{0}\right)+m\left(h^{0}\right)
\end{array}\right.
\end{array}
$$

where $\tilde{l}_{R}^{ \pm}=\tilde{e}_{R}^{ \pm}, \tilde{\mu}_{R}^{ \pm}, \tilde{\tau}_{1}^{ \pm}$and $\tilde{l}_{L}^{ \pm}=\tilde{e}_{L}^{ \pm}, \tilde{\mu}_{L}^{ \pm}, \tilde{\tau}_{2}^{ \pm}$. In region B there are three-body decays via off-shell particles and two-body decays via on-shell right-handed sleptons possible. The relevant decays are

- via on-shell right-handed slepton $\tilde{e}_{R}, \tilde{\mu}_{R}$ or $\tilde{\tau}_{1}$

$$
\tilde{\chi}_{2}^{0} \rightarrow \tilde{l}_{R}^{ \pm} l^{\mp} \rightarrow \tilde{\chi}_{1}^{0} l^{+} l^{-} ; \quad \tilde{\chi}_{1}^{ \pm} \rightarrow \tilde{l}_{R}^{ \pm} \nu_{l} \rightarrow \tilde{\chi}_{1}^{0} l^{ \pm} \nu_{l}
$$

- via off-shell $W^{ \pm *}$ or $Z^{0 *}$ boson

$$
\tilde{\chi}_{2}^{0} \rightarrow \tilde{\chi}_{1}^{0} Z^{0 *} \rightarrow \tilde{\chi}_{1}^{0}\left\{\begin{array} { l } 
{ l ^ { + } l ^ { - } } \\
{ q \overline { q } } \\
{ \nu \overline { \nu } }
\end{array} \quad \tilde { \chi } _ { 1 } ^ { \pm } \rightarrow \tilde { \chi } _ { 1 } ^ { 0 } W ^ { \pm * } \rightarrow \tilde { \chi } _ { 1 } ^ { 0 } \left\{\begin{array}{c}
{ }^{ \pm} \nu_{l} \\
q_{1} \bar{q}_{2}
\end{array}\right.\right.
$$

- via off-shell sneutrino $\tilde{\nu}_{e}, \tilde{\nu}_{\mu}$ or $\tilde{\nu}_{\tau}$

$$
\tilde{\chi}_{2}^{0} \rightarrow \tilde{\nu}_{l}^{*} \bar{\nu}_{l} \rightarrow \tilde{\chi}_{1}^{0} \nu_{l} \bar{\nu}_{l} ; \quad \tilde{\chi}_{1}^{ \pm} \rightarrow \tilde{\nu}_{l}^{*} l^{ \pm} \rightarrow \tilde{\chi}_{1}^{0} l^{ \pm} \nu_{l}
$$

As a decay via on-shell particle becomes available the decay channel becomes dominant. In region B this is the decay via right-handed on-shell sleptons as soon as there is enough phase space for the decay products. If there is not enough phase space for the products of the decay via an on-shell slepton, which is the case at the border of region $\mathrm{A}$ to $\mathrm{B}$, the decays via off-shell particles, mostly sleptons, have a significant branching ratio.

It is shown in appendix B, that the combination of PYTHIA and ISAJET does not handle mass widths correctly. As a result in a band of approximately $4 \mathrm{GeV}$ around the transition area from three-body decay via off-shell particles to sequential two-body decay via on-shell particles the branching ratios for the used decay channels are artificial and do not represent the predictions by mSUGRA. 


\section{Region C: Decay via on-shell right-handed sleptons and sneutrinos dominant}

Region $\mathrm{C}$ is defined by the mass relations

$$
\begin{gathered}
m\left(\tilde{l}_{R}^{ \pm}\right)<m(\tilde{\nu})<m\left(\tilde{\chi}_{2}^{0}\right)<m\left(\tilde{l}_{L}^{ \pm}\right) \\
m\left(\tilde{l}_{R}^{ \pm}\right)<m(\tilde{\nu})<m\left(\tilde{\chi}_{1}^{ \pm}\right)<m\left(\tilde{l}_{L}^{ \pm}\right) \\
m\left(\tilde{\chi}_{2}^{0}\right)<\left\{\begin{array}{l}
m\left(\tilde{\chi}_{1}^{0}\right)+m\left(Z^{0}\right) \\
m\left(\tilde{\chi}_{1}^{0}\right)+m\left(h^{0}\right)
\end{array} ; m\left(\tilde{\chi}_{1}^{ \pm}\right)<m\left(\tilde{\chi}_{1}^{0}\right)+m\left(W^{ \pm}\right)\right.
\end{gathered}
$$

where $\tilde{l}_{R}^{ \pm}=\tilde{e}_{R}^{ \pm}, \tilde{\mu}_{R}^{ \pm}, \tilde{\tau}_{1}^{ \pm}$and $\tilde{l}_{L}^{ \pm}=\tilde{e}_{L}^{ \pm}, \tilde{\mu}_{L}^{ \pm}, \tilde{\tau}_{2}^{ \pm}$. In region $\mathrm{C}$ there are off-shell three-body decays and decays via on-shell right-handed sleptons possible. The relevant decays are

- via on-shell sneutrino $\tilde{\nu}_{e}, \tilde{\nu}_{\mu}$ or $\tilde{\nu}_{\tau}$

$$
\tilde{\chi}_{2}^{0} \rightarrow \tilde{\nu}_{l} \bar{\nu}_{l} \rightarrow \tilde{\chi}_{1}^{0} \nu_{l} \bar{\nu}_{l} ; \quad \tilde{\chi}_{1}^{ \pm} \rightarrow \tilde{\nu}_{l} l^{ \pm} \rightarrow \tilde{\chi}_{1}^{0} l^{ \pm} \nu_{l}
$$

- via on-shell right-handed slepton $\tilde{e}_{R}, \tilde{\mu}_{R}$ or $\tilde{\tau}_{1}$

$$
\tilde{\chi}_{2}^{0} \rightarrow \tilde{l}_{R}^{ \pm} l^{\mp} \rightarrow \tilde{\chi}_{1}^{0} l^{+} l^{-} ; \quad \tilde{\chi}_{1}^{ \pm} \rightarrow \tilde{l}_{R}^{ \pm} \nu_{l} \rightarrow \tilde{\chi}_{1}^{0} l^{ \pm} \nu_{l}
$$

- via off-shell $W^{ \pm *}$ or $Z^{0 *}$ boson

$$
\tilde{\chi}_{2}^{0} \rightarrow \tilde{\chi}_{1}^{0} Z^{0 *} \rightarrow \tilde{\chi}_{1}^{0}\left\{\begin{array} { l } 
{ l ^ { + } l ^ { - } } \\
{ q \overline { q } } \\
{ \nu \overline { \nu } }
\end{array} \quad \tilde { \chi } _ { 1 } ^ { \pm } \rightarrow \tilde { \chi } _ { 1 } ^ { 0 } W ^ { \pm * } \rightarrow \tilde { \chi } _ { 1 } ^ { 0 } \left\{\begin{array}{l}
l^{ \pm} \nu_{l} \\
q_{1} \bar{q}_{2}
\end{array}\right.\right.
$$

In region $\mathrm{C}$ on-shell decays via right-handed sleptons and sneutrinos are available. Which of the two on-shell decay channels is dominant depends on the mixing of the chargino and neutralino.

It has to be noted that the division into regions is only valid up to a couple of GeV. For example at the transition from region $\mathrm{A}$ to $\mathrm{B}$ the border is at $m\left(\tilde{\chi}_{2}^{0}\right)=m\left(\tilde{\tau}_{1}\right)$ even though for the third generation decay the decay changes from off-shell slepton, $W^{ \pm *}$ or $Z^{0 *}$ boson to on-shell slepton is at $m\left(\tilde{\chi}_{2}^{0}\right)=m\left(\tilde{\tau}_{1}^{ \pm}\right)+m\left(\tau^{\mp}\right)$. At the transition from region A to B the region border is similar for all three generations; at the transition from region $\mathrm{B}$ to $\mathrm{C}$ a difference between first or second and third generation slepton to sneutrino transition is visible. 


\subsubsection{Cross Section for Associated Chargino-Neutralino Production}

We calculate the cross section for associated chargino-neutralino production in $\sqrt{s}=1.96 \mathrm{TeV}$ $p \bar{p}$ collisions at the Tevatron with PROsPINo 2.0[35 $]^{6}$. It was shown in section 5.1, that the main dependence of the cross section for associated chargino-neutralino production is on $m_{1 / 2}$. Figure 5.22 shows the cross section in the $\left(m_{0}, m_{1 / 2}\right)$-plane.

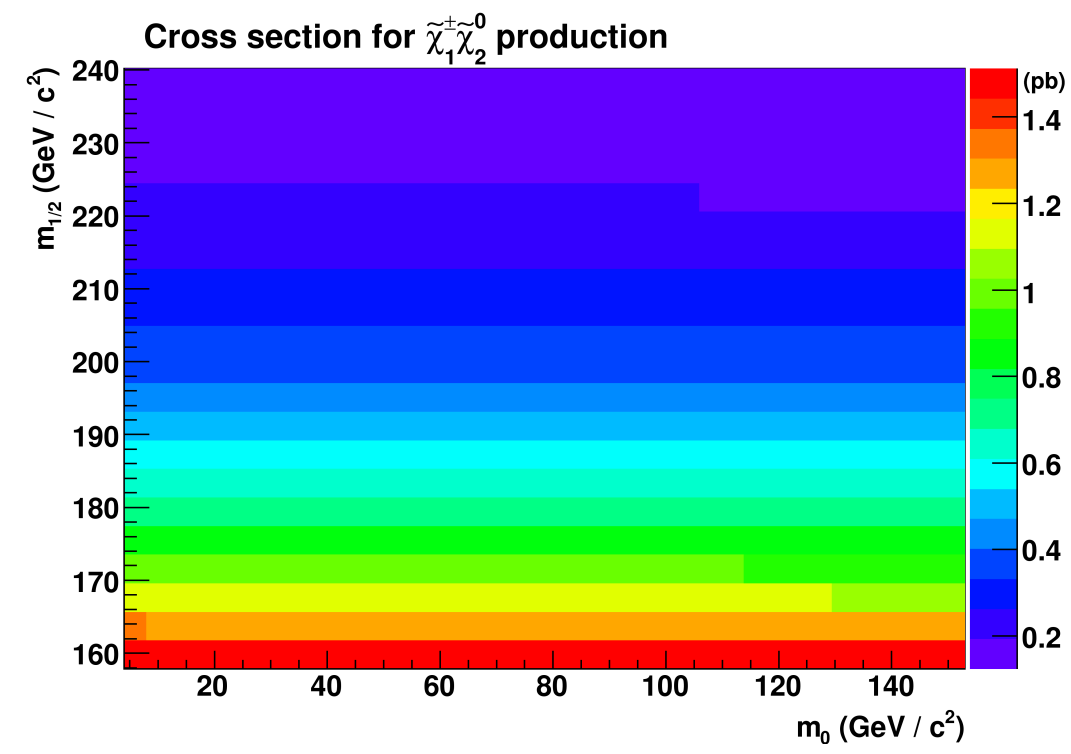

Figure 5.22: Cross section for the production of an associated chargino-neutralino pair in $\sqrt{s}=1.96 \mathrm{TeV} p \bar{p}$ collisions at the Tevatron as a function of $m_{0}$ and $m_{1 / 2}$.

\subsubsection{Branching Ratio of $\tilde{\chi}_{1}^{ \pm} \tilde{\chi}_{2}^{0}$ into Three Leptons}

Together with the masses, which affect the cross section limit, the branching ratio of the produced chargino-neutralino pair into three leptons and the cross section for its production are the most important quantities for an exclusion of parts of the mSUGRA parameter space. As the cross section for the production has a rather simple dependence on the mSUGRA parameters $m_{0}$ and $m_{1 / 2}$ the branching ratio into three leptons is the major factor that is determining the sensitivity of the discussed trilepton analysis.

The branching ratio can be seen in Fig. 5.23. Starting in region A for high $m_{0}$ the decay is dominated by the exchange of an off-shell $W^{ \pm *}$ or $Z^{0 *}$ boson. As this decay can lead

\footnotetext{
${ }^{6}$ The particle spectrum used was produced with ISAJET $7.75[33]$.
} 


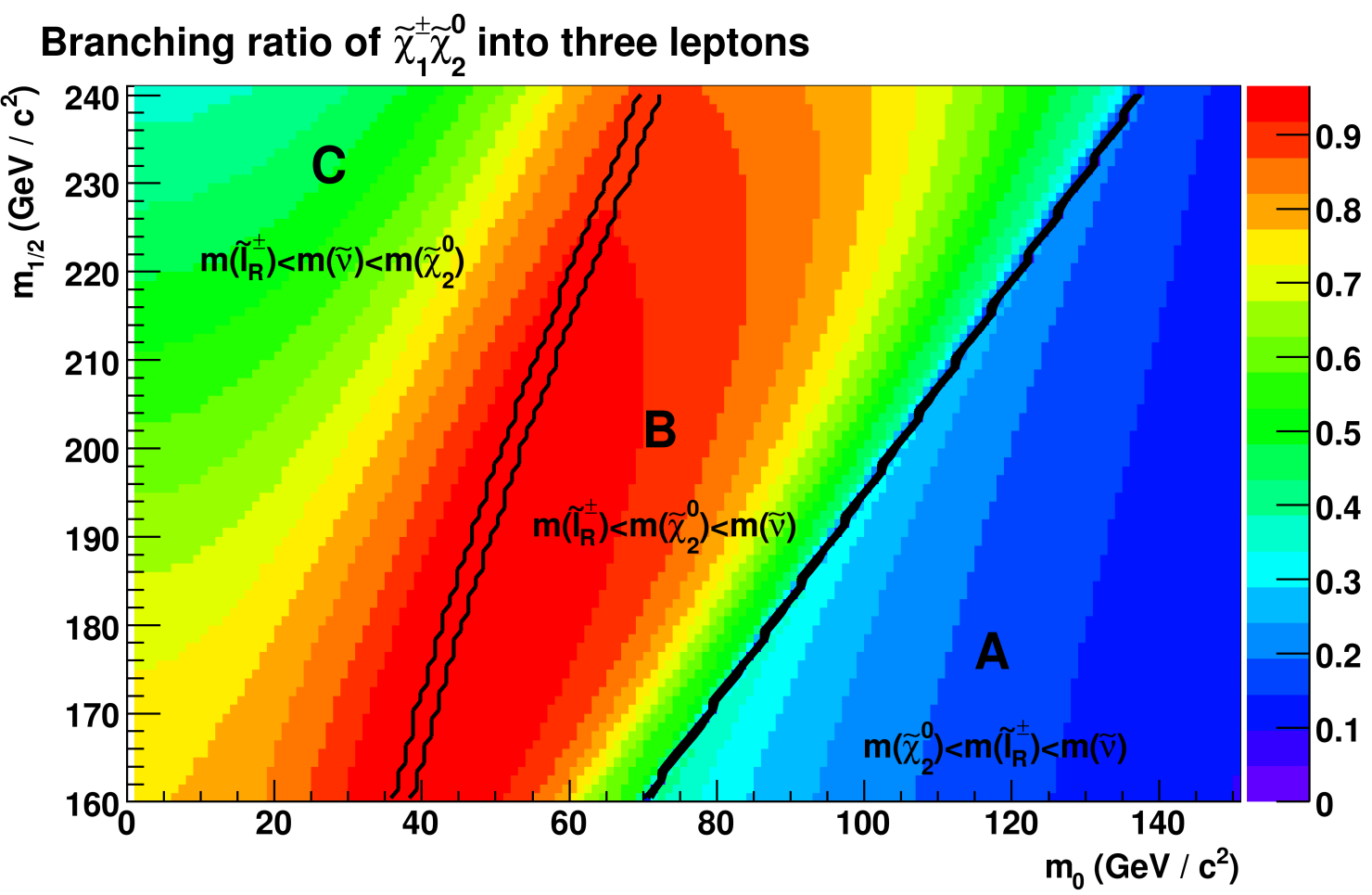

Figure 5.23: Branching ratio for the decay of a chargino-neutralino pair into three leptons, where leptons are electrons, muons and $\tau$ leptons.

to final states with quarks, neutrinos and leptons the branching ratio into three leptons is comparably low and a lot of events have quarks in the final state ${ }^{7}$. Decays into quarks are not detectable with a trilepton signature. Close to the line where $m\left(\tilde{\chi}_{2}^{0}\right) \approx m\left(\tilde{\chi}_{1}^{ \pm}\right) \approx m\left(\tilde{l}_{R}^{ \pm}\right)$ the decay via off-shell slepton becomes more important. As this decay goes into leptons with $100 \%$ branching ratio, the branching ratio of $\tilde{\chi}_{1}^{ \pm} \tilde{\chi}_{2}^{0}$ into three leptons increases. At the border of region $\mathrm{A}$ to $\mathrm{B}$ the branching ratio of $\tilde{\chi}_{1}^{ \pm} \tilde{\chi}_{2}^{0}$ into three leptons drops for a width at the order of $1 \mathrm{GeV}$. This is an artefact of the combination of ISAJET and PYTHIA. For a more detailed description of the effect see appendix B. In region B the decay via on-shell right-handed sleptons is dominant. As this decay has $100 \%$ leptons in the final state, the branching ratio into three leptons increases. In region $\mathrm{C}$ the decay via on-shell sneutrinos becomes available and the branching ratio decreases again. From the point of the branching ratio the exclusion limit should be optimal in region $\mathrm{B}$.

\footnotetext{
${ }^{7}$ For details see also Fig. 5.2.
} 


\subsubsection{Average Number of $\tau$ Leptons per Event}

Even though the branching ratio into three leptons favors region B for exclusion, the average number of $\tau$ leptons disfavors region B. It can be seen in section 4.6.3 that the purity of the channels with three leptons is significantly better than the purity of the channels with two leptons and one track. The average number of $\tau$ leptons is a measure for the ratio of the number of events in the two channel categories.

In Fig. $\mathrm{C}$ it can be seen that the average number of $\tau$ leptons per event in region $\mathrm{B}$ is significantly higher. The reason is that the chargino is a mixed mass eigenstate which is dominated by the Higgsino gauge eigenstate. The coupling of the Higgsino to the third generation $\tilde{\tau}_{1}$ slepton is significantly higher than the coupling to the selectron $\tilde{e}_{R}$ and smuon $\tilde{\mu}_{R}$. As a decay via $\tilde{\tau}_{1}$ slepton yields $\tau$ leptons the average number of $\tau$ leptons increases.

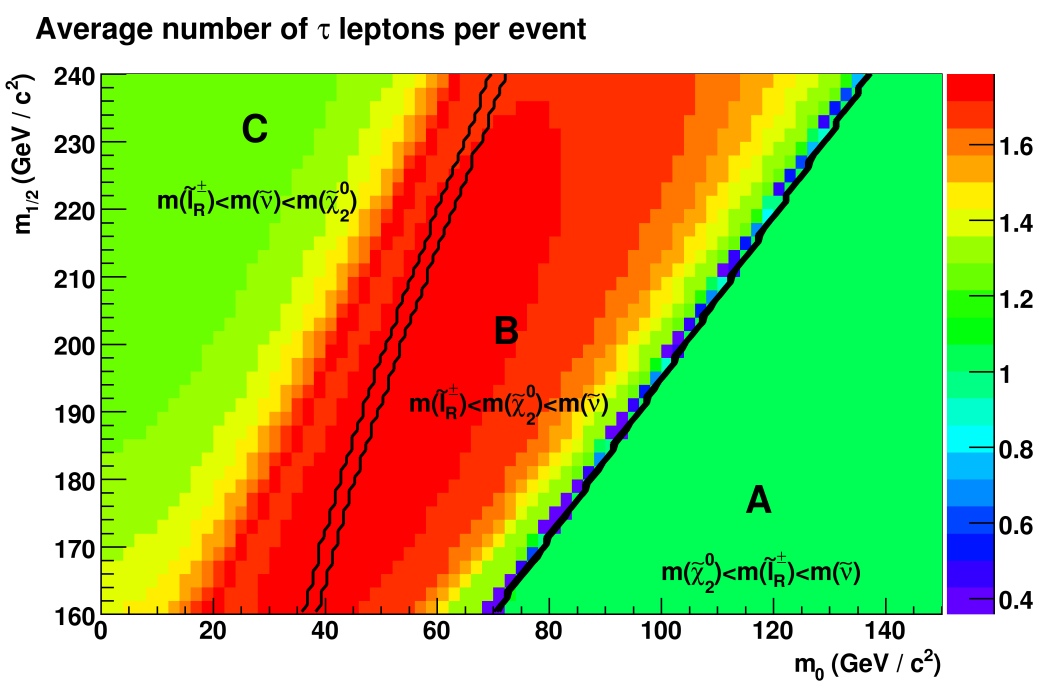

Figure 5.24: The mean number of $\tau$ leptons per event is a measure for the number of events in the trilepton versus the number of events in the dilepton + track channel. The drop in the mean number of $\tau$ leptons at the border of region A to B is due to the artefact of the setup of PYTHIA and ISAJET discussed in appendix B. 


\subsection{Limits on the Production Cross Section and the Chargino Mass}

Within errors the results of the search for trileptons in section 4.6.3 have been consistent with the predictions for the standard model background. As no deviations from the Standard Model have been found, it is possible to obtain an observed upper limit on the cross section multiplied by the branching ratio of $\tilde{\chi}_{1}^{ \pm} \tilde{\chi}_{2}^{0}$ into three leptons in mSUGRA. The observed upper limit is the maximal cross section times braching ratio that is consistent at $95 \%$ confidence level with the number of observed events.

\subsubsection{Calculation of a Limit on the Production Cross Section}

Based on the results from section 4.6 .3 a $95 \%$ confidence level limit on the cross section

and branching ratio for the process $\tilde{\chi}_{2}^{0} \tilde{\chi}_{1}^{ \pm} \rightarrow 3$ leptons, where a lepton can be an electron, a muon or a $\tau$ lepton, was calculated. As suggested by the CDF Statistics Committee[57] we use the MCLIMIT software[58] to calculate confidence levels.

This software is using the frequentist definition of a probability and is comparing the signal + background hypothesis $H_{1}$ ("Supersymmetry is realized in nature") to the nullhypothesis $H_{0}$ (background only, "Supersymmetry is not realized in nature"). If $s_{i}$ is the expected signal, $b_{i}$ the expected background and $d_{i}$ the observed number of events in in channel $\mathrm{i}$ according to the Poisson distribution the likelihood ratio for channel i $Q_{i}$ is defined as

$$
Q_{i}=\frac{P\left(H_{1} \mid \text { data }\right)}{P\left(H_{0} \mid \text { data }\right)}=\frac{\exp -\left(s_{i}+b_{i}\right)\left(s_{i}+b_{i}\right)^{d_{i}}}{d_{i} !} / \frac{\exp -\left(b_{i}\right)\left(b_{i}\right)^{d_{i}}}{d_{i} !}
$$

and the combined likelihood ratio as

$$
Q=\prod_{i} Q_{i}
$$

The confidence level for excluding the signal + background hypothesis is

$$
C L_{s+b}=P_{s+b}\left(Q<Q_{\mathrm{obs}}\right)
$$

and similarly $C L_{b}$ for excluding the null-hypothesis. For this analysis the modified frequency confidence levels

$$
C L_{s}=\frac{C L_{s+b}}{C L_{b}}
$$


are used. In the following the cross section multiplied by branching ratio into three leptons $\tilde{\chi}_{1}^{ \pm} \tilde{\chi}_{2}^{0} \rightarrow 3$ leptons for which $C L_{s}\left(\sigma\left(\tilde{\chi}_{1}^{ \pm} \tilde{\chi}_{2}^{0}\right) \times \operatorname{BR}\left(\tilde{\chi}_{1}^{ \pm} \tilde{\chi}_{2}^{0} \rightarrow 3\right.\right.$ leptons $\left.)\right)=0.05$, the $95 \%$ confidence level, is determined.

\subsubsection{Limit on the Production Cross Section}

In order to set a limit on the cross section for the production of $\tilde{\chi}_{1}^{ \pm} \tilde{\chi}_{2}^{0} 87$ Monte Carlo points were produced. The supersymmetric particle spectrum from ISAJET 7.51[33] is used to generate events in PYTHIA 6.216[31]. $\tau$ decays are treated by TAUOLA[34]; full detector and trigger simulation is done with the CDF software MCPRODUCTION 6.1.4mc. For all Monte Carlo points we use mSUGRA with $\tan \beta=3, A_{0}=0 \mathrm{GeV}, \mu>0$ and varied $m_{0}$ and $m_{1 / 2}$. The values of $m_{0}$ and $m_{1 / 2}$ for the produced Monte Carlo samples can be obtained from Fig. 5.25. As stated in section 5.3.1 the MCLIMIT software[58] was used to calculate a 95\% confidence level upper limit for the cross section multiplied with the branching ratio of $\tilde{\chi}_{1}^{ \pm} \tilde{\chi}_{2}^{0}$ into three leptons.

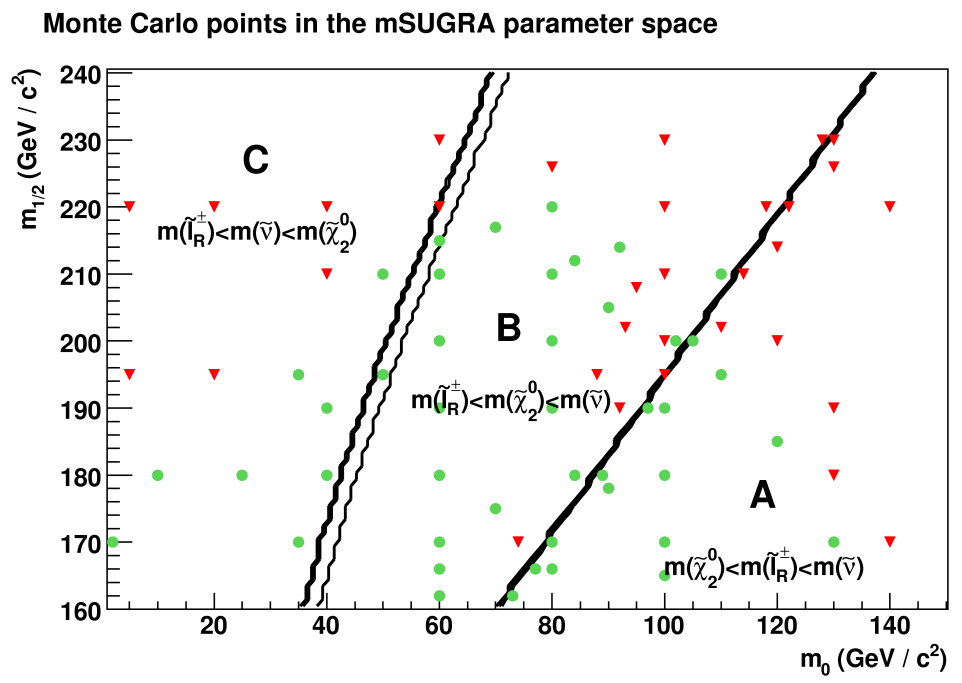

Figure 5.25: Monte Carlo points used for obtaining a limit on the $\tilde{\chi}_{1}^{ \pm} \tilde{\chi}_{2}^{0}$ production cross section. For the red points supersymmetry does not manifest itself at this set of parameters; for the green points exclusion is not possible.

The observed upper limit on the cross section for chargino-neutralino production multiplied by the branching ratio of their decay into three leptons (lepton $=\mathrm{e}, \mu, \tau)$ set by this 
analysis can be found in Fig. 5.26. The observed limit on the cross section decouples the effects of the branching ratio and the production cross section from kinematic effects ${ }^{8}$ on the limit set by this analysis. Through the kinematic properties of the event the observed limit is indirectly dependent on mSUGRA. For a more detailed explanation of the features of Fig. 5.26 see section 5.3.3.

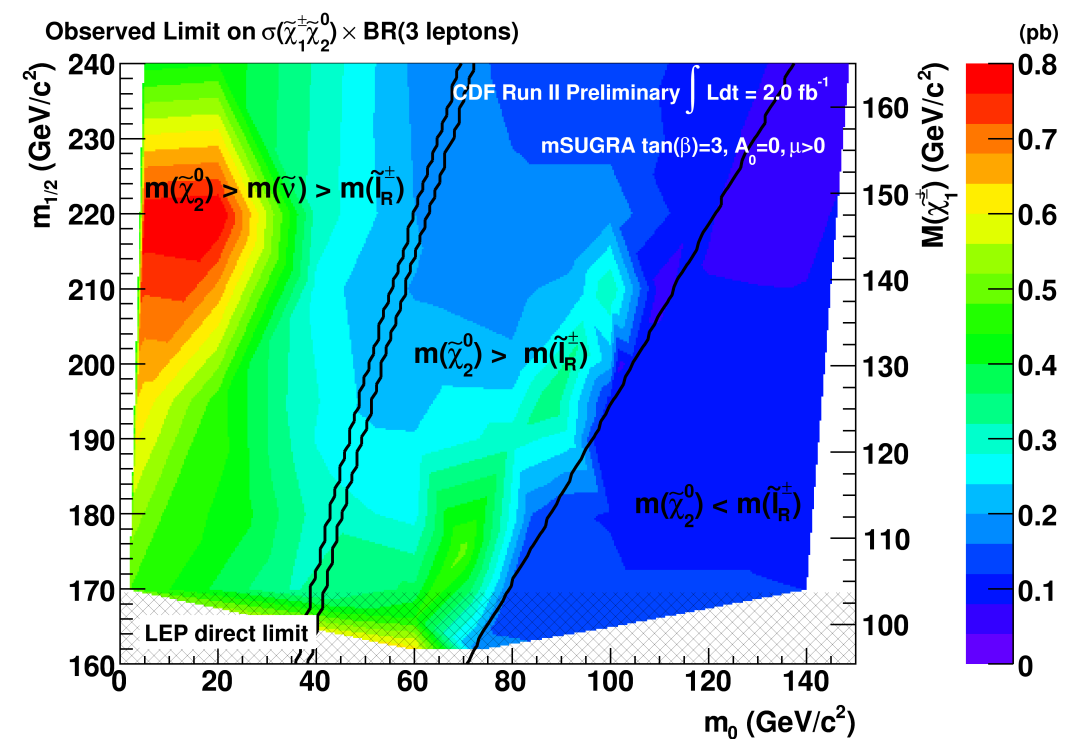

Figure 5.26: Observed limit on the production cross section multiplied with the branching ratio of $\tilde{\chi}_{1}^{ \pm} \tilde{\chi}_{2}^{0}$ into three leptons, where leptons are electrons, muons and $\tau$ leptons.

\footnotetext{
${ }^{8}$ The observed upper limit on $\sigma \times \mathrm{BR}$ is still including effects from the number of events in the trilepton and the dilepton + track channels and thus the mean number of $\tau$ leptons in the final state.
} 


\subsubsection{Limit on the Chargino Mass}

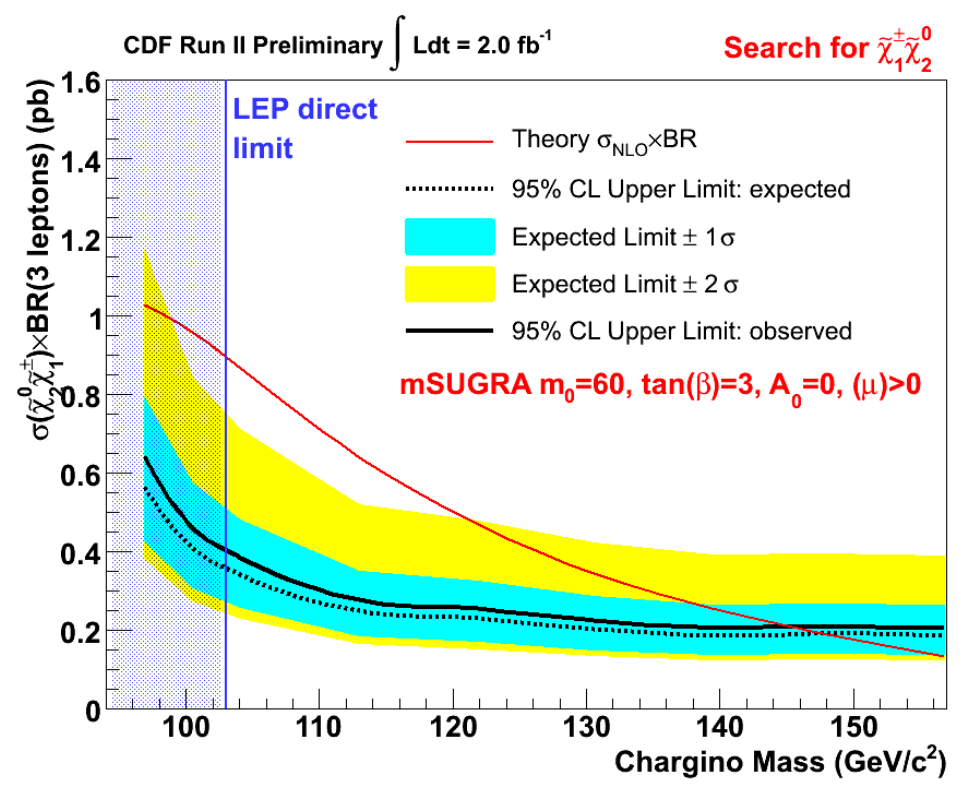

Figure 5.27: Limit on the mass of the chargino at $m_{0}=60 \mathrm{GeV} / \mathrm{c}^{2}$ in region $\mathrm{B}$, where two-body decays are dominant.

As the branching ratio of $\tilde{\chi}_{1}^{ \pm} \tilde{\chi}_{2}^{0}$ into three leptons and the cross section for the production of chargino and neutralino are determinded by the mSUGRA parameters, an exclusion in mSUGRA can be calculated. The theory cross section was calculated with PROSPINO 2.0[35]; the theoretical branching ratio of chargino and neutralino into three leptons was calculated with PYTHia 6.409[31]. Both programs use the particle spectrum of ISAJET 7.75[33] as an input.

In Fig. 5.27 the theory cross section times branching ratio from mSUGRA and the observed upper limit is plotted as a function of $m_{1 / 2}$ at benchmark point BP1 in region $\mathrm{B}$, where the decay is dominantly via on-shell right-handed sleptons. This analysis is able to exclude that supersymmetry manifests itself at all parameter points where the upper limit is lower than the theoretical quantity. In Fig. 5.27 it is possible to exclude chargino masses below approximately $145 \mathrm{GeV}$ for $m_{0}=60 \mathrm{GeV}, \tan \beta=3, A_{0}=0 \mathrm{GeV}, \mu>0$. The collider LEP has set a limit on the chargino mass based on its center-of-mass energy at $m\left(\tilde{\chi}_{1}^{ \pm}\right)=103.5 \mathrm{GeV} / \mathrm{c}^{2}[59]$; the limit is shown. 


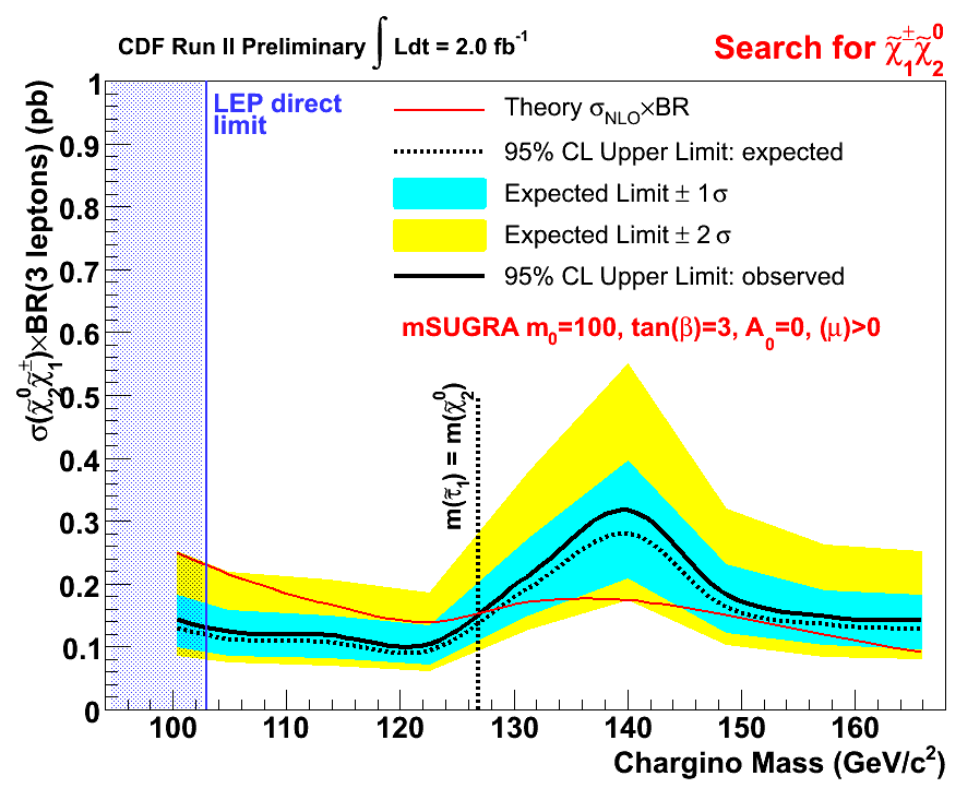

Figure 5.28: Limit on the mass of the chargino at $m_{0}=100 \mathrm{GeV} / \mathrm{c}^{2}$ in region $\mathrm{A}$, where three-body decays are dominant.

In Fig. 5.28 the corresponding plot can be seen as a function of $m_{1 / 2}$ at $m_{0}=100 \mathrm{GeV} / \mathrm{c}^{2}$, $\tan \beta=3, A_{0}=0 \mathrm{GeV}$ and $\mu>0$. Up to approximately $127 \mathrm{GeV} / \mathrm{c}^{2}$ this is in region $\mathrm{A}$, where the decay is dominantly via off-shell particles $W^{ \pm *}, Z^{0 *}, \tilde{l}_{R}^{ \pm *}$. Here the observed limit and the theoretical expectation follow the shape from Fig. 5.27, but are at lower $\sigma \times$ BR. The cross section at same values of $m_{1 / 2}$ should be comparable, but the branching ratio into three leptons is significantly lower in this regions which leads to a lower theory expectation. At $m_{\tilde{\chi}_{1}^{ \pm}}>127 \mathrm{GeV} / \mathrm{c}^{2}$ the observed upper limit increases. The decay here is mostly via an on-shell slepton and as the slepton mass is approximately the mass of the neutralino there is very small phase space for the lepton produced in the decay

$$
\tilde{\chi}_{2}^{0} \rightarrow \tilde{l}_{R}^{ \pm} l^{\mp}
$$

The probability for the lepton not to pass the minimum requirements on the lepton transverse momentum ${ }^{9}$ increases and and so does the observed upper limit. Once the phase space

\footnotetext{
${ }^{9}$ For the $p_{T}$ requirements of the analysis see section 4.3 .
} 
for the produced lepton is big enough, more leptons pass the minimum transverse momentum requirements and the observed limit is decreases again. The limit on the chargino mass here is approximately $127 \mathrm{GeV} / \mathrm{c}^{2}$.

It has to be noted that these limits are only valid for four fixed parameters: $m_{0}, \tan \beta$, $A_{0}$ and $\mu$. It was shown in section 5.1 that the parameters most important for the masses of the important supersymmetric particles are $m_{0}$ and $m_{1 / 2}$. In what follows we relax the requirement that $m_{0}$ is fixed and examine the sensitivity of the analysis for varying $m_{0}$ and $m_{1 / 2}$, but fixed $\tan \beta=3, A_{0}=0 \mathrm{GeV}$ and $\mu>0$. Figure 5.25 documents the Monte Carlo points that we have produced. It can be seen that the Monte Carlo points are not in a regular grid; in section 5.2.1 several regions with different phenomenology have been introduced and we followed the shape of these regions.

For every Monte Carlo point the observed upper limit on $\sigma \times \mathrm{BR}$ was determined. The quantity

$$
X=\frac{\sigma \times B R(\text { Observed limit })-\sigma \times \mathrm{BR}(\text { Theory })}{\sigma \times B R(\text { Theory })}
$$

is interpolated by Delauney triangulation with ROOT and is plotted in Fig. 5.29. The region where $X<0$ can be excluded and is displayed in Fig. 5.30. It can be seen that the

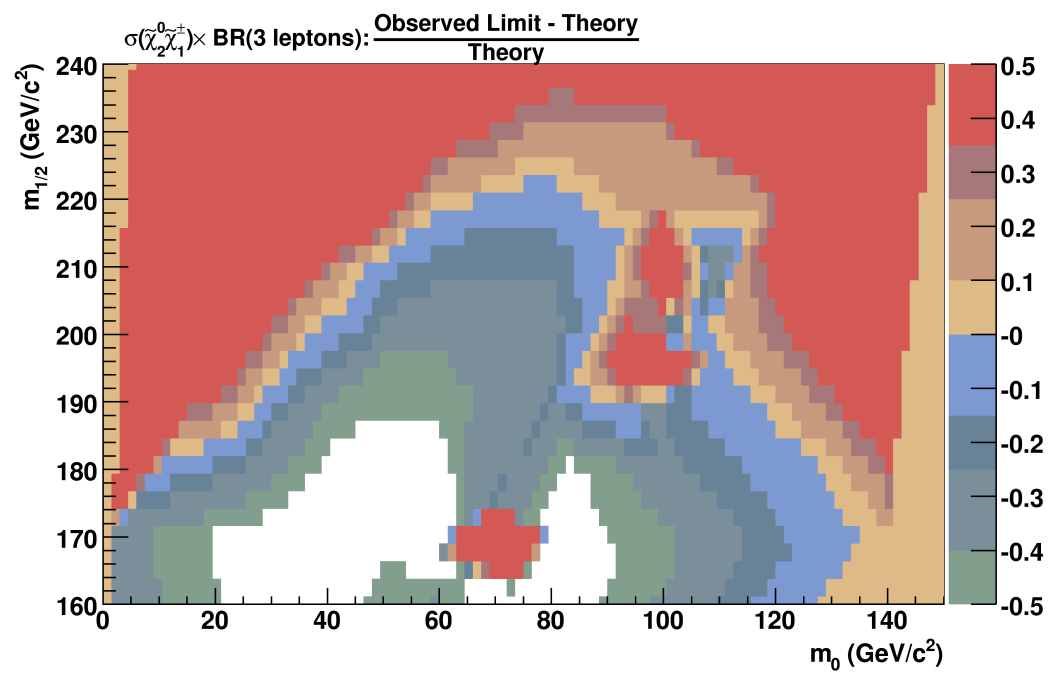

Figure 5.29: Interpolation of the observed upper limit and the theory cross section and branching ratio into three leptons to obtain an exclusion region in mSUGRA

exclusion region is split into two parts with a strip of approximately 10 to $15 \mathrm{GeV}$, where no exclusion can be claimed. Following the explanation for Fig. 5.28 a soft lepton that can not 
always pass the $p_{T}$ cuts is produced and the sensitivity of the analysis worsens. The exact value of the observed upper limit for the cross section is highly dependent on a fine change in the parameters $m_{0}$ and $m_{1 / 2}$, so that the interpolation is not reliable and an exclusion can not be claimed in this regions.

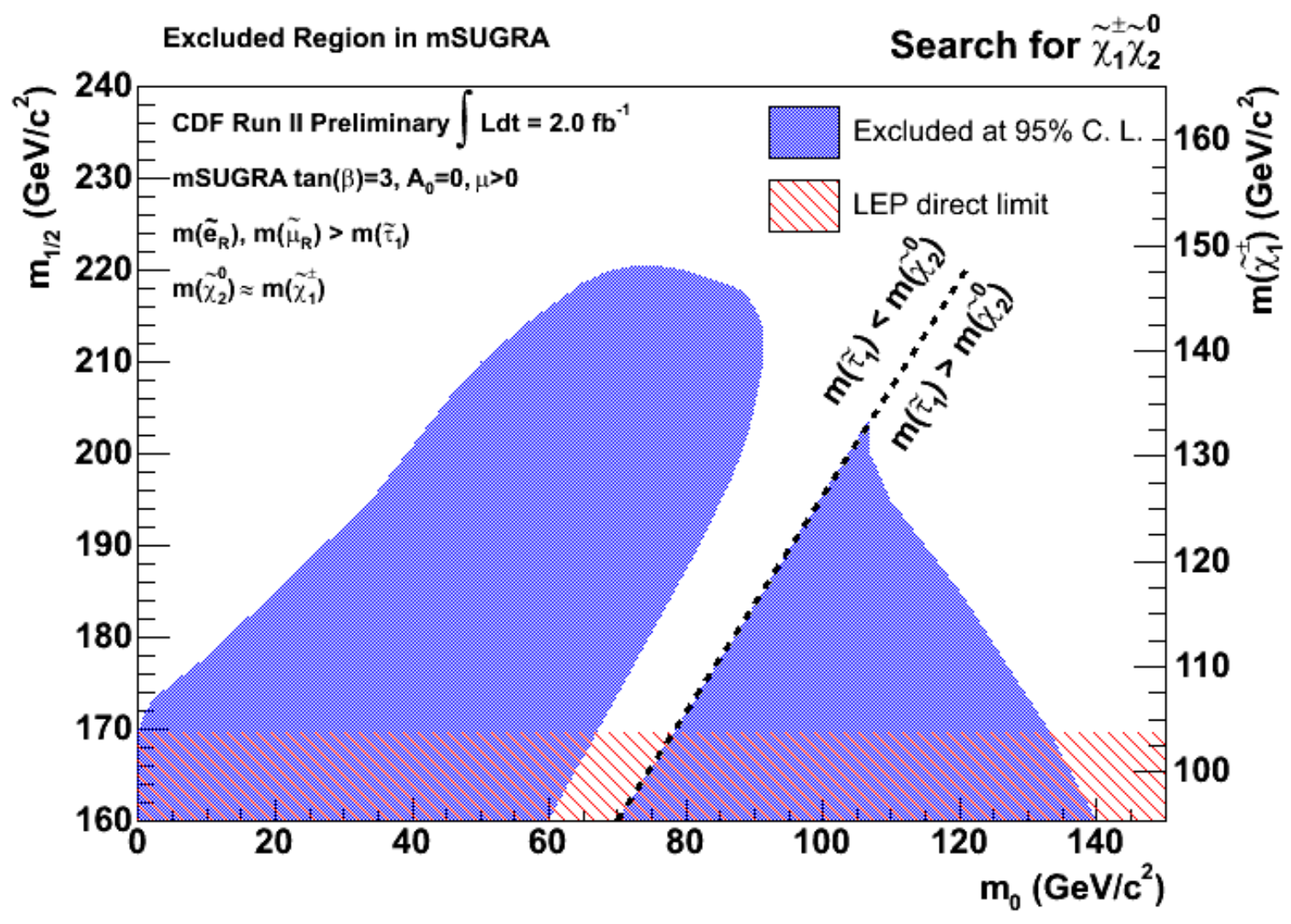

Figure 5.30: 95\% confidence level exclusion region in mSUGRA

Nevertheless a search for two leptons with equal charge should have sensitivity in this strip. In $50 \%$ of all events the soft lepton has a different charge than the other two leptons. As one soft leptons implies two harder leptons a search for like-sign leptons that is specifically designed to look for supersymmetry in this strip can search for two hard leptons with same charge. The standard model background should be low and preliminary studies have shown such an analysis has sensitivity in the biggest part of this strip.

Figure 5.31 shows an overlay of the theoretical production cross section multiplied by the branching ratio of $\tilde{\chi}_{1}^{ \pm} \tilde{\chi}_{2}^{0}$ into three leptons and the exclusion region in mSUGRA. It can be seen that except at the border of region $\mathrm{A}$ and $\mathrm{B}$ the exclusion region follows the contour of $\sigma \times \mathrm{BR}$. This can be taken as a proof that the important quantities for the exclusion 
are the branching ratio of $\tilde{\chi}_{1}^{ \pm} \tilde{\chi}_{2}^{0}$ into three leptons and the production cross section. The kinematics of the event have an impact on the rather smooth observed upper limit at the border of regions where small mass differences exist only.

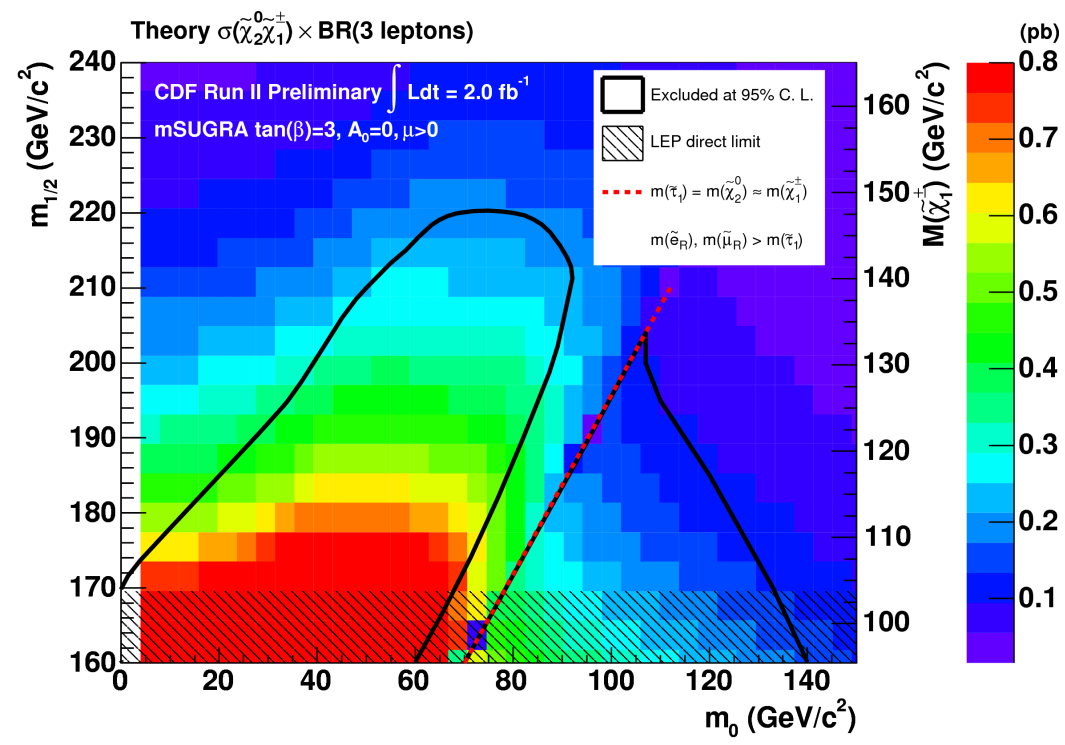

Figure 5.31: Overlay of the theoretical production cross section multiplied by the branching ratio of $\tilde{\chi}_{1}^{ \pm} \tilde{\chi}_{2}^{0}$ into three leptons and the exclusion region in mSUGRA

\subsection{Comparison of the Results to Previous Searches}

Searches for supersymmetry have been carried out at several high energy collider experiments. At the Tevatron both CDF and D $\varnothing$ have been looking for chargino and neutralino production; however, most of the current limits on supersymmetric models are derived by analyses using data of the collider LEP.

\subsubsection{Results of LEP2}

The Joint SUSY Working Group by the experiments ALEPH, DELPHI, L3 and OPAL has published a combination of the searches for supersymmetry and other limiting searches of the individual experiments[59]. The searches most relevant for this analysis are

- Combined LEP Chargino Results for low DM,

- Combined LEP Chargino Results for large $m_{0}$, 
- Combined LEP Selectron/Smuon/Stau Results,

- Interpretation of the results in Minimal SUGRA.

All four LEP experiments have searched for charginos in the decay channel into leptons, leptons+jets and jets. In the combined LEP Chargino Results for large $m_{0}$ the limits are combined under the assumption that the chargino decays via a $W^{ \pm *}$ boson. It can be seen in section 5.1.1 that this is the case for $m_{0} \gg 100 \mathrm{GeV} / \mathrm{c}^{2}$ in the parameter space considered in this thesis, but for lower $m_{0}$ the decay via on- or off-shell sleptons may be dominant. Overall for $m\left(\tilde{\nu}_{l}\right)>300 \mathrm{GeV} / \mathrm{c}^{2}$ the exclusion limit from $206-208 \mathrm{GeV}$ data is

$$
m\left(\tilde{\chi}_{1}^{ \pm}\right)>103.5 \mathrm{GeV} / \mathrm{c}^{2}
$$

The condition $m\left(\tilde{\nu}_{l}\right)>300 \mathrm{GeV} / \mathrm{c}^{2}$ is fulfilled for high $m_{0}$, but might not be fulfilled for small $m_{0}$.

The search for chargino with low DM, where $D M=m\left(\tilde{\chi}_{1}^{ \pm}\right)-m$ (LSP), is done in the MSSM framework. The published limits for $0 \mathrm{GeV} / \mathrm{c}^{2}<D M<10 \mathrm{GeV} / \mathrm{c}^{2}$ and high $m_{0}$ or $\mu \ll M_{2}$ are

$$
m\left(\tilde{\chi}_{1}^{ \pm}\right)>92.4 \mathrm{GeV} / \mathrm{c}^{2}\left(\text { for } \mu \ll M_{2}\right) ; \quad m\left(\tilde{\chi}_{1}^{ \pm}\right)>91.9 \mathrm{GeV} / \mathrm{c}^{2}\left(\text { for high } m_{0}\right) .
$$

The LEP search for right-handed sleptons has also published limits. The exclusion regions for the interpretation of the LEP results in mSUGRA can be found in Fig. 5.32.

\subsubsection{CDF Results}

CDF has published previous results with approximately $1 \mathrm{fb}^{-1}$ of data in the like-sign channels $e^{ \pm} e^{ \pm}, e^{ \pm} \mu^{ \pm}$and $\mu^{ \pm} \mu^{ \pm}[60]$, the non-exclusive high $p_{T}$ channels eel, e $\mu l, \mu \mu l$ and $\mu e l[61]$, the low $p_{T}$ channel $\mu \mu l[62]$ and a trilepton channel including a track eeT[63]. A combination of the channels can be found in [64].

A limit in the mSUGRA model for $m_{0}=60 \mathrm{GeV}, \tan \beta=3, A_{0}=0 \mathrm{GeV}, \mu>0$ and varying $m_{1 / 2}$ is shown in Fig. 5.33. It was not possible to derive a limit on the chargino mass. CDF also presents limits in a scenario without slepton mixing and same couplings for all slepton generations. mSUGRA with the same set of parameters as above is used 
ADLO Preliminary - $\mu>0, \mathrm{M}_{\mathrm{lop}}=175 \mathrm{GeV} / \mathrm{c}^{2}, \mathrm{~A}_{0}=0$
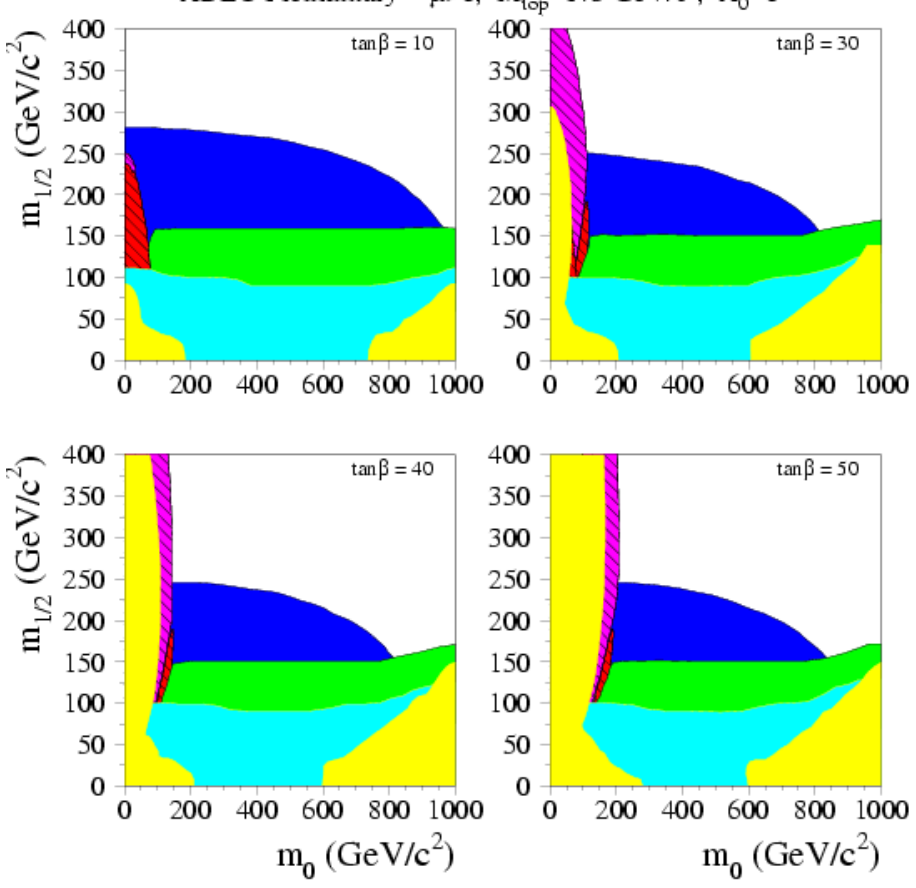

ADLO Preliminary - $\mu<0, M_{10 p}=175 \mathrm{GeV} / \mathrm{c}^{2}, \mathrm{~A}_{0}=0$
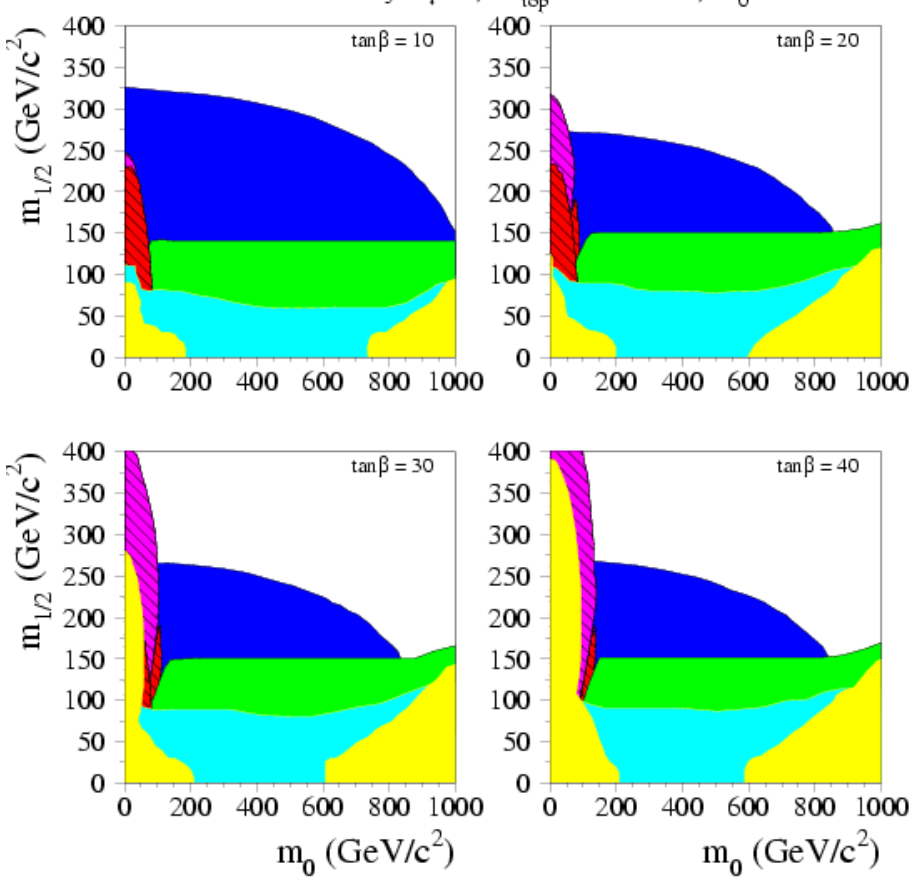

No Minimal SUGRA solution: no EWSB or tachyonic particles;

Regions inconsistent with the measurement of the electroweak parameters at LEP1;

Regions excluded by chargino searches;

Regions excluded by selectron or stau standard searches:

Regions excluded by the search for $\mathrm{hZ}$;

Regions excluded by the search for heavy stable charged particles applied to staus.

Figure 5.32: Excluded regions by the LEP experiments in the $\left(m_{0}, m_{1 / 2}\right)$-plane of mSUGRA for fixed $\tan \beta, A_{0}, \operatorname{sgn}(\mu)$. 
and slepton mixing in the mixing matrix used by SOFTSUSY is turned off. This procedure decreases the number of $\tau$ leptons in the final state and leads to a higher acceptance of the analysis. A limit of $m\left(\tilde{\chi}_{1}^{ \pm}\right)>129 \mathrm{GeV}$ can be derived for this special set of parameters. In a model with $\tan \beta=3, A_{0}=0 \mathrm{GeV}, \mu>0$ and

$$
\begin{array}{r}
m\left(\tilde{\chi}_{1}^{ \pm}\right)=m\left(\tilde{\chi}_{2}^{0}\right)=m\left(\tilde{\chi}_{1}^{0}\right) \\
\operatorname{BR}\left(\tilde{\chi}_{1}^{ \pm} \rightarrow \tilde{\chi}_{1}^{0} l^{ \pm} \nu_{l}\right)=\operatorname{BR}\left(W^{ \pm} \rightarrow l^{ \pm} \nu_{l}\right) \\
\operatorname{BR}\left(\tilde{\chi}_{2}^{0} \rightarrow \tilde{\chi}_{1}^{0} l^{+} l^{-}\right)=\operatorname{BR}\left(Z \rightarrow l^{+} l^{-}\right)
\end{array}
$$

the limit on $\sigma \times \mathrm{BR}$ is shown in [64], but it was not possible to derive a limit on the chargino mass.
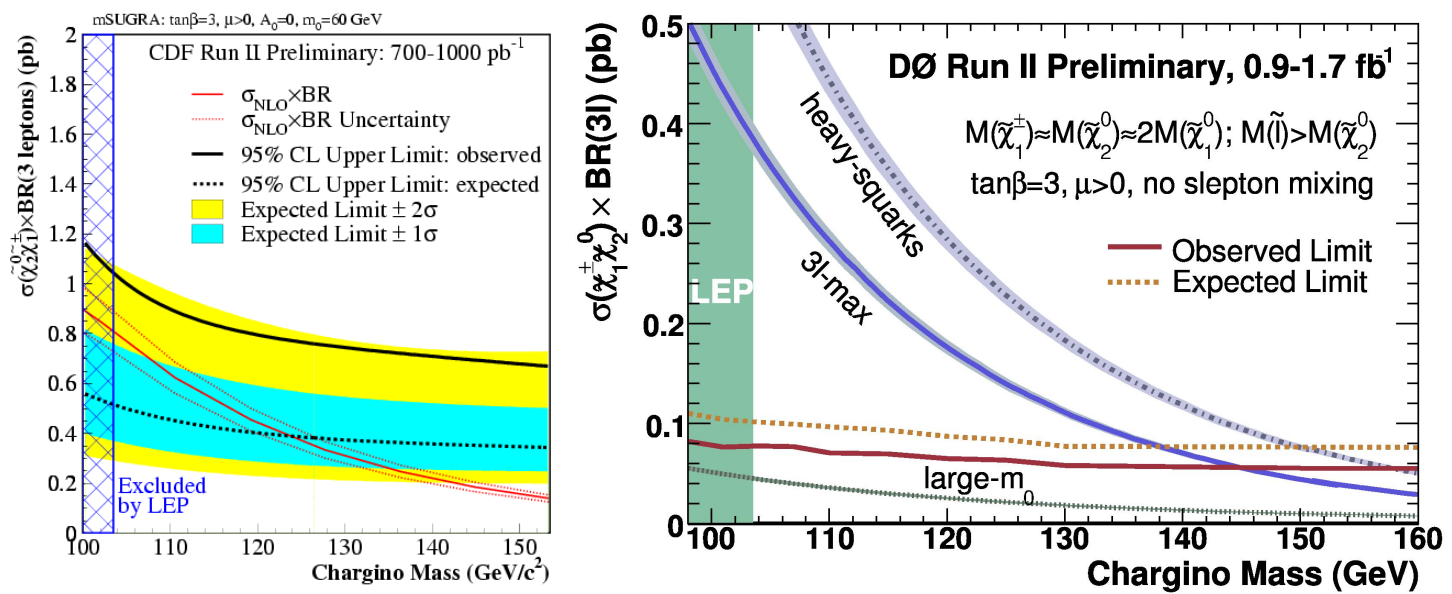

Figure 5.33: Excluded chargino masses for CDF and DØ. CDF was using plain mSUGRA, whereas DØ was working in a mSUGRA inspired MSSM scenario with no slepton mixing.

\subsubsection{DØ Results}

A similar search for chargino and neutralino production in a trilepton final state was done by $\mathrm{D} \varnothing[65,66]$ with up to $1.7 \mathrm{fb}^{-1}$ of data. DØ is presenting results in a mSUGRA inspired MSSM scenario, where slepton mixing is turned off; it is assumed that all three slepton generation have the same couplings and

$$
m\left(\tilde{\chi}_{1}^{ \pm}\right) \approx m\left(\tilde{\chi}_{2}^{0}\right) \approx 2 \times m\left(\tilde{\chi}_{1}^{0}\right)
$$

For the 3l-max scenario the chargino and slepton mass parameters are chosen in a way that $m(\tilde{l})>m\left(\tilde{\chi}_{2}^{0}\right)$ and the branching ratio $\operatorname{BR}\left(\tilde{\chi}_{2}^{0} \tilde{\chi}_{1}^{ \pm} \rightarrow 3\right.$ leptons $)$ is maximized. In 
practice the maximization of the branching ratio is achieved by $\operatorname{setting} m\left(\tilde{l}_{R}^{ \pm}\right) \gtrsim m\left(\tilde{\chi}_{2}^{0}\right)$. This requirement ensures that the decay is dominantly via off-shell sleptons. Similar to the no slepton mixing scenario used by CDF these requirements lead to less $\tau$ leptons in the final state and thus a higher acceptance. The heavy squarks scenario squarks are set to high masses to increase the production cross section of chargino-neutralino pairs ${ }^{10}$. Additionally a scenario with high $m_{0}$, where the chargino and neutralino decay is dominantly via an off-shell $W^{ \pm *}$ or $Z^{0 *}$ boson, is shown. As the branching ratio into leptons is significantly smaller in this scenario no limit on the chargino mass can be derived. The derived limits for the 3l-max and the heavy slepton scenario can be seen in Fig. 5.33.

It should be noted that the previous CDF and DØ results are only applicable for a very restrained set of supersymmetric models and choice of parameters. It is tried to generalize the results obtained in this thesis to a broader range of supersymmetric models and sets of parameters in chapter 6 .

\footnotetext{
${ }^{10}$ See section 4.1 for details.
} 


\section{Chapter 6}

\section{Model-Independent Interpretation of the Results}

\subsection{Description of the Method}

Section 5.4 shows that the results of CDF and D $\varnothing$ have always been interpreted in a specific model. Furthermore the models have major differences, ranging from plain mSUGRA to the MSSM scenario of DØ, where several other assumptions are used to maximize the sensitivity. The development of a model-independent description of the analysis results is investigated in this section.

Limits on the cross section as calculated in section 5.3 are dependent on

1. the expected Standard Model and non-physics background and the observed number of events in data and

2. the acceptance of the analysis for the supersymmetric signal.

Whereas the expected background and the observed number of events in data are completely model-independent and depend only on the analysis, the acceptance of the analysis for the supersymmetric signal is naturally dependent on the model for which limits are to be derived.

The acceptance itself is dependent on the type and the kinematics of the reconstructed analysis objects. For the acceptance the difference between a reconstructed electron and a reconstructed muon is negligible, but electrons or muons and isolated tracks have different purities. Since for signal tracks mainly originate from decays of $\tau$ leptons, the ratio of the 
number of events with i $\tau$ leptons $(i=0,1,2,3)$ and the number of all events with 3 leptons plays an important role. We define this ratio as

$$
\begin{aligned}
F_{i} & =\frac{\operatorname{BR}\left(\tilde{\chi}_{1}^{ \pm} \tilde{\chi}_{2}^{0} \rightarrow i \tau+(3-i) l_{e / \mu}+X\right)}{\operatorname{BR}\left(\tilde{\chi}_{1}^{ \pm} \tilde{\chi}_{2}^{0} \rightarrow 3 l+X\right)}= \\
& =\frac{\text { Number of trilepton events with i } \tau \text { leptons }}{\text { Number of total trilepton events }},
\end{aligned}
$$

where $l=e, \mu, \tau$ and $l_{e / \mu}=e, \mu$. If it is possible to obtain the acceptance $A_{i}$ of a signal sample that is only consisting of events with i $\tau$ leptons in the final state, the overall acceptance is

$$
A=\sum_{i=0}^{3} F_{i} A_{i} .
$$

It was shown earlier in this thesis that the acceptance of the analysis is dependent on the mass spectrum of the involved particles. In this section we will restrict us to the cases

1. $m\left(\tilde{\chi}_{1}^{0}\right)<m\left(\tilde{\chi}_{1}^{ \pm}\right) \approx m\left(\tilde{\chi}_{2}^{0}\right)<$ mass of all other supersymmetric particles

2. $m\left(\tilde{\chi}_{1}^{0}\right)<m\left(\tilde{l}_{R}^{ \pm}\right)<m\left(\tilde{\chi}_{1}^{ \pm}\right) \approx m\left(\tilde{\chi}_{2}^{0}\right)<$ mass of all other supersymmetric particles, where $\tilde{l}_{R}$ stands for the lighter selectron, smuon and stau.

These assumptions include that $m\left(\tilde{\chi}_{1}^{ \pm}\right) \approx m\left(\tilde{\chi}_{2}^{0}\right)$, which is the case in several supersymmetric models. Case 2 here additionally includes the assumption that the sleptons are mass degenerate. This assumption is different from the assumption of $\mathrm{D} \varnothing$ as we explicitly treat the couplings of the stau differently than the coupling of the selectron or smuon. Considering Eqn. 2.20 this is a major improvement over the model used by DØ. At benchmark point BP1 the difference in mass between stau and selectron or smuon is $0.1 \mathrm{GeV}^{1}$. If the mass difference of the electron or muon and $\tau$ lepton is also considered, for a neutralino the decay via a $\tilde{\tau}$ slepton would even be disfavored over the decay via $\tilde{e}$ or $\tilde{\mu}$. This could hardly explain the differences in branching ratio seen in Fig. 5.2. The difference of the branching ratio of the decay via a stau slepton and the decay via selectron or smuon is predominantly due to the fact of the different couplings and not the difference in mass. The effects of the coupling can fully be accounted for by the branching ratios $F_{i}, i=0,1,2,3$.

Using these assumptions the relevant part of the mass spectrum is fully described by

\footnotetext{
${ }^{1}$ See Table 5.2 for details.
} 
1. the chargino mass $m\left(\tilde{\chi}_{1}^{ \pm}\right)$and thus implicitly the mass of the neutralino,

2. the LSP mass $m\left(\tilde{\chi}_{1}^{0}\right)$,

3. the mass of the lighter sleptons $m\left(\tilde{l}_{R}^{ \pm}\right)$.

An equivalent parameterization that is closer to the experimentally relevant quantities uses the parameters

1. chargino mass $m\left(\tilde{\chi}_{1}^{ \pm}\right)$and thus implicitly the mass of the neutralino,

2. $\Delta M_{1}=m\left(\tilde{\chi}_{1}^{ \pm}\right)-m\left(\tilde{l}_{R}^{ \pm}\right)$,

3. $\Delta M_{2}=m\left(\tilde{\chi}_{1}^{ \pm}\right)-m\left(\tilde{\chi}_{1}^{0}\right)$.

As we don't consider the case where a slepton is the $\mathrm{LSP}^{2}$, it is $\Delta M_{1}<\Delta M_{2}$.

If it is possible to measure the acceptance of the final states split up according to their $\tau$ content $A_{i}$ as a function of $m\left(\tilde{\chi}_{1}^{ \pm}\right), \Delta M_{1}, \Delta M_{2}$, general limits dependent on

- the masses $m\left(\tilde{\chi}_{1}^{ \pm}\right), \Delta M_{1}, \Delta M_{2}$

- the branching ratios $F_{i}, i=0,1,2,3$

can easily be derived for other models by using Eqn. 6.3.

\subsection{Determination of the Acceptance Functions}

In mSUGRA, $m\left(\tilde{\chi}_{1}^{0}\right) \approx m\left(\tilde{\chi}_{2}^{0}\right)^{3}$. For technical reasons we have reduced the set of mass parameters in section 6.1 to $m\left(\tilde{\chi}_{1}^{ \pm}\right)$and $\Delta M_{1}=m\left(\tilde{\chi}_{1}^{ \pm}\right)-m\left(\tilde{l}_{R}^{ \pm}\right) ; \Delta M_{2}$ is determined by the relations of mSUGRA. A way to produce samples where all three parameters are free, is described in appendix D.

As full CDF detector simulation is very CPU time consuming, we use PYTHIA 6.409[31] and ISASUgRA 7.75[33] to produce Monte Carlo samples. The decay of the $\tau$ lepton is done by TAUOLA 2.1[34]. We use mSUGRA and vary $m_{0}$ and $m_{1 / 2}$ in order to get samples with

\footnotetext{
${ }^{2}$ A case with a charged particle as the LSP would not explain the WMAP dark matter observations.

${ }^{3}$ For details see section 2.47 .
} 
different mass parameters. $A_{0}=0 \mathrm{GeV}, \tan \beta=3$ and $\mu>0$ are kept constant. For every considered realization of supersymmetry ${ }^{4}$ we produce four Monte Carlo subsamples which consist of events where $\tilde{\chi}_{2}^{0} \tilde{\chi}_{1}^{ \pm}$are forced to decay into three leptons among which $i=0,1,2,3$ leptons are $\tau$ leptons. No further detector simulation is used. In section 6.3 we show that at the level of precision needed for this model-independent approach standalone PYTHIA without full CDF detector simulation is sufficient.

In the PyThia Monte Carlo samples we select events in a way to mimic the trilepton analysis described in section 4. Similar to the analysis channels in Table 4.6 we select events with three leptons or two leptons and one isolated $\operatorname{track}^{5}$ (lepton=e, $\mu$ ) passing the criteria documented in Table 6.1. Missing $E_{T}$ is calculated by summing the vector components of the transverse momentum of neutrinos and LSP's in the event. The acceptance is defined

\begin{tabular}{|c|c|}
\hline Variable & Selection \\
\hline \hline$p_{\mathrm{T}}^{1,2,3}$ & $>15,5,5 \mathrm{GeV}$ \\
$\left|\eta^{1,2,3}\right|$ & $<1.1$ \\
$\mathscr{E}_{T}$ & $>20 \mathrm{GeV}$ \\
$m_{O S}^{1}$ & $>20 \mathrm{GeV}, \notin[76 \mathrm{GeV}, 106 \mathrm{GeV}]$ \\
$m_{O S}^{2}$ & $>13 \mathrm{GeV}, \notin[76 \mathrm{GeV}, 106 \mathrm{GeV}]$ \\
\hline
\end{tabular}

Table 6.1: Selection criteria for the determination of the acceptance in the modelindependent approach. $m_{O S}^{i}, i=1,2$ are the two invariant masses of oppositely charged particles that can be obtained from the three analysis objects.

by

$$
A_{i}=\frac{\text { Number of events that are able to pass the cuts }}{\text { Total number of events }}
$$

and determined individually for the four Monte Carlo samples. We show the acceptance split up into subsamples in Fig. 6.1. It has to be noted that the current trilepton analysis has very small acceptance to events with 2 or more $\tau$ leptons as the analysis currently only uses trilepton and dilepton + track channels. Including channels with more than one track would result in a considerable increase of background.

\footnotetext{
${ }^{4} \mathrm{~A}$ realization of supersymmetry here is fully defined by the mass parameters.

${ }^{5}$ Similar to the isolation requirement in section 4 we require that the sum of $p_{T}$ of all other tracks in a cone of 0.4 in the $(\eta, \phi)$-plane around the considered track is less than $10 \%$ of the track's $p_{T}$.
} 
Acceptance : $0 \tau$ 's

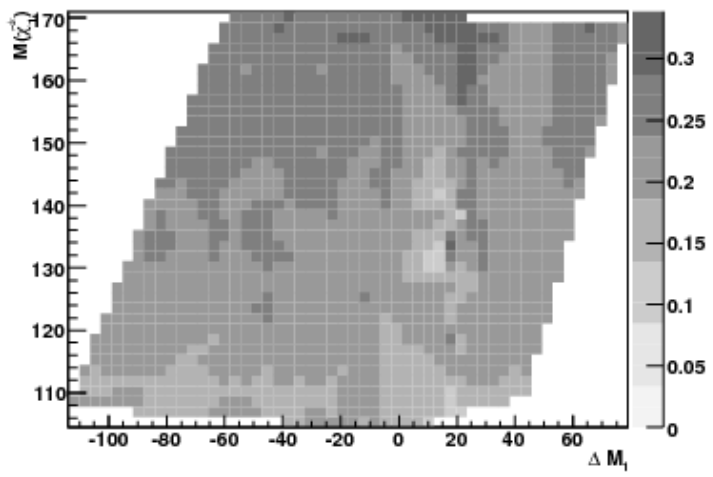

Acceptance : 2 's

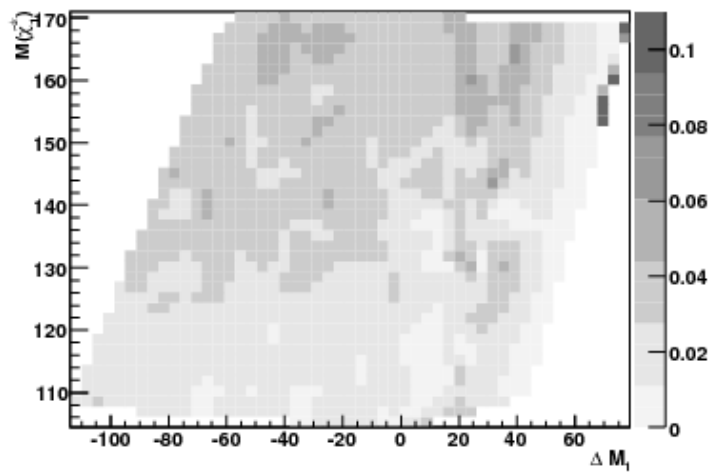

Acceptance : $1 \tau$ 's

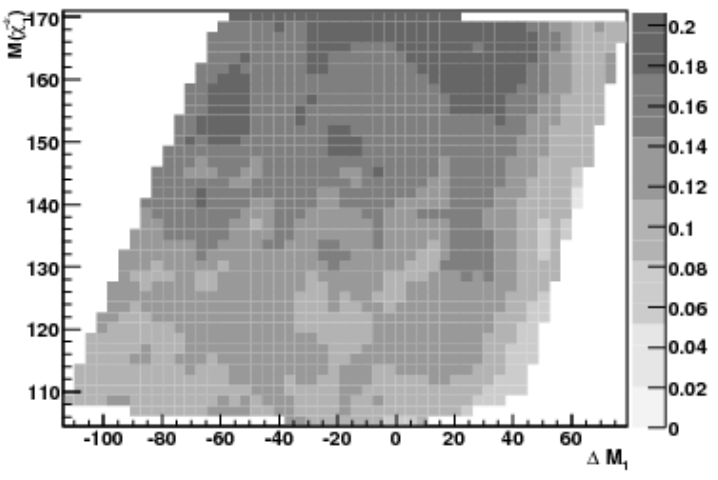

Acceptance : 3 's

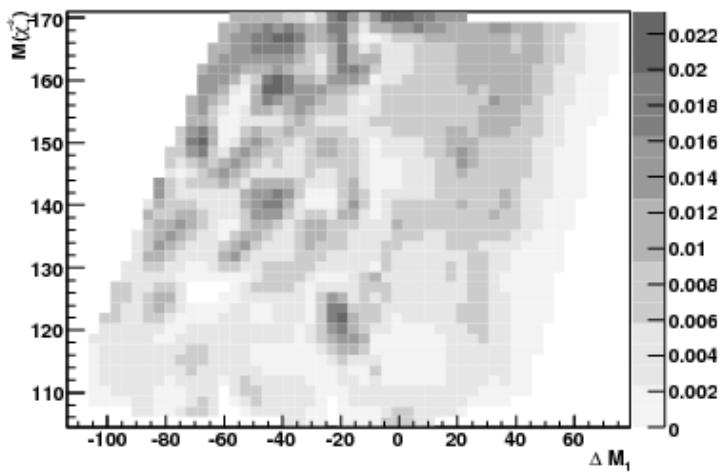

Figure 6.1: Analysis acceptance split into channels according to the number of $\tau$ leptons in the trilepton final state 
It can be seen that the acceptance is a smooth function unless the mass differences $\Delta M_{1}=m\left(\tilde{\chi}_{1}^{ \pm}\right)-m\left(\tilde{l}_{R}^{ \pm}\right)$or $m\left(\tilde{\chi}_{1}^{ \pm}\right)-m(\tilde{\nu})$ are small. For small mass differences new decay channels open up. Additionally soft leptons are produced and sophisticated detector simulation becomes important; standalone PYTHIA Monte Carlo is not reliable there. We ensure that $m(\tilde{\nu})>m\left(\tilde{\chi}_{1}^{ \pm}\right)$and $\Delta M_{1}<-2 \mathrm{GeV}$ or $\Delta M_{1}>15 \mathrm{GeV}$ to avoid these regions. In the regions of smooth behavior we fit the acceptance with a function of the type

$$
A=p_{0}+p_{1}\left(\frac{\mathrm{M}\left(\tilde{\chi}_{1}^{ \pm}\right)}{100 \mathrm{GeV}}\right)+p_{2}\left(\frac{\mathrm{M}\left(\tilde{\chi}_{1}^{ \pm}\right)}{100 \mathrm{GeV}}\right)^{2} .
$$

The results for the individual subsamples can be found in Table 6.2. The fits approximate the measured acceptance within 20\%. A comparison of the fit of the acceptance and the acceptance itself for various sets of mSUGRA parameters can be found in Table 6.3.

\begin{tabular}{|c|ccc|}
\hline Subsample & $p_{0}$ & $p_{1}$ & $p_{2}$ \\
\hline \hline $0 \tau$ 's & -0.19 & 0.49 & -0.14 \\
$1 \tau$ 's & -0.044 & 0.14 & 0 \\
$2 \tau$ 's & -0.12 & 0.19 & -0.055 \\
$3 \tau$ 's & -0.008 & 0.01 & 0 \\
\hline
\end{tabular}

Table 6.2: The values of the parameters of the acceptance fits in the different subsamples. The fit function is given by Eqn. 6.5.

\begin{tabular}{|cccc|c|c|}
\hline$m_{0}$ & $m_{1 / 2}$ & $A_{0}$ & $\tan \beta$ & Actual Acc. & Calc. Acc. \\
\hline \hline 60 & 190 & 0 & 3 & 0.08810 & 0.08000 \\
60 & 190 & -200 & 3 & 0.07840 & 0.07160 \\
70 & 190 & 100 & 5 & 0.08040 & 0.07532 \\
70 & 180 & 0 & 10 & 0.03210 & 0.03066 \\
70 & 180 & 200 & 10 & 0.03880 & 0.03801 \\
120 & 180 & 200 & 3 & 0.11590 & 0.11322 \\
120 & 180 & -200 & 5 & 0.12850 & 0.12183 \\
120 & 180 & 200 & 10 & 0.10550 & 0.10923 \\
1000 & 200 & 0 & 10 & 0.15890 & 0.15250 \\
1000 & 200 & -200 & 10 & 0.16300 & 0.15421 \\
1000 & 200 & 200 & 10 & 0.16160 & 0.15250 \\
\hline
\end{tabular}

Table 6.3: Comparison of the actual acceptance from Pythia (Actual Acc.) and the acceptance calculated using the fits (Calc. Acc.) for several sets of mSUGRA points. It is $\mu>0$ for all points. 
The acceptance from full detector simulation Monte Carlo and standalone PYTHIA Monte Carlo is different. To account for this difference we introduce a scale factor of

$$
\frac{A_{\mathrm{CDF}}}{A_{\text {Pythia }}}=0.169
$$

for the region where $m\left(\tilde{l}_{R}^{ \pm}\right)<m\left(\tilde{\chi}_{1}^{ \pm}\right)$and

$$
\frac{A_{\mathrm{CDF}}}{A_{\text {Pythia }}}=0.222
$$

for the region where $m\left(\tilde{l}_{R}^{ \pm}\right)>m\left(\tilde{\chi}_{1}^{ \pm}\right)$. The scale factor for the light slepton region $\mathrm{B}^{6}$ in Eqn. 6.6 was measured at the mSUGRA point $m_{0}=60 \mathrm{GeV} / \mathrm{c}^{2}, m_{1 / 2}=190 \mathrm{GeV} / \mathrm{c}^{2}$, $\tan \beta=3, A_{0}=0 \mathrm{GeV}, \mu>0$ and the scale factor at the heavy slepton region $\mathrm{A}^{6}$ in Eqn. 6.6 $m_{0}=100 \mathrm{GeV} / \mathrm{c}^{2}, m_{1 / 2}=180 \mathrm{GeV} / \mathrm{c}^{2}, \tan \beta=3, A_{0}=0 \mathrm{GeV}, \mu>0$.

\subsection{Verification of the Proposed Method in mSUGRA}

To test the procedure proposed in section 6.2 we apply it to mSUGRA. We split our total trilepton acceptance into five channels using the same ratios as the trilepton analysis in chapter 4 for the two mass conditions. The background estimates from Table 4.10 are used to calculate limits on cross section times branching ratio according to section 5.3.1. The limits are compared to theory cross section obtained with PROSPINO 2.0[35] and PYTHIA 6.409[31] and the exclusion region in Fig. 6.2 is derived. Comparison to Fig. 5.30 shows that the proposed method is applicable.

\footnotetext{
${ }^{6}$ For the definition of the regions see section 5.2.1.
} 


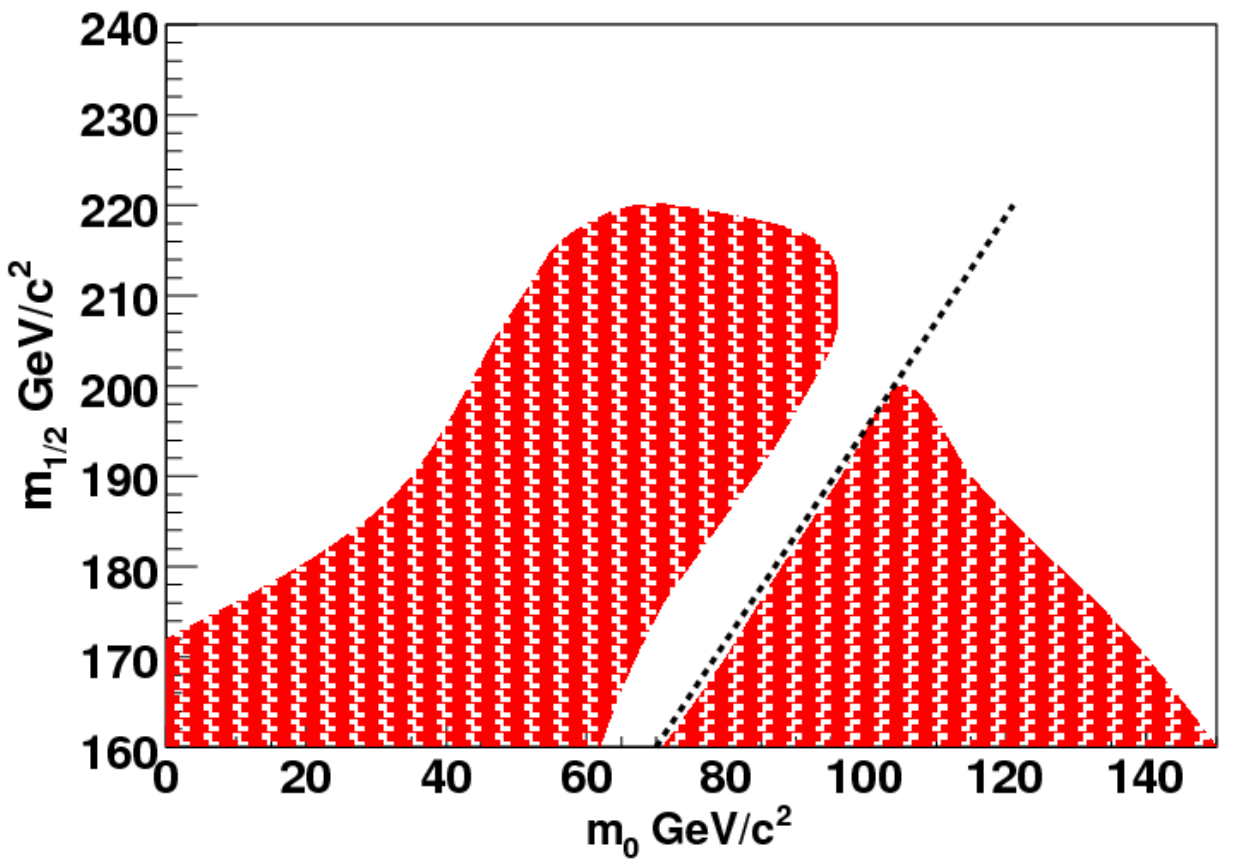

Figure 6.2: Exclusion region in mSUGRA obtained with the proposed model-independent approach. Comparison to Fig. 5.30 shows the applicability of the proposed method. 


\section{Chapter 7}

\section{Summary}

Supersymmetry is a proposed symmetry that relates bosons and fermions and - if it is realized in nature - can answer some of the open questions of the Standard Model of Particle Physics. As supersymmetric particles have not yet been observed, supersymmetry has to be broken. mSUGRA is a model of supergravity where supersymmetry breaking is mediated by gravity. Due to its simplicity mSUGRA is widely used as a benchmark to evaluate searches for supersymmetry.

In this thesis a search for associated chargino-neutralino production in $\sqrt{s}=1.96 \mathrm{TeV}$ center-of-mass energy $p \bar{p}$ collision at the Tevatron in $2 \mathrm{fb}^{-1}$ of data from the Collider Detector at Fermilab (CDF II) is documented. The leptonic decay of chargino and neutralino is mostly via on- or off-shell sleptons or off-shell $W^{ \pm *} / Z^{0 *}$ bosons into leptons following

$$
\tilde{\chi}_{1}^{ \pm} \tilde{\chi}_{2}^{0} \rightarrow \tilde{\chi}_{1}^{0} l^{ \pm} \nu_{l} \tilde{\chi}_{1}^{0} l^{+} l^{-}, \quad \text { where } \quad l=e, \mu, \tau
$$

If R-parity is conserved, the lightest supersymmetric particle $\tilde{\chi}_{1}^{0}$ is stable and together with the weakly interacting neutrino provides missing transverse energy. The final state three leptons and missing transverse energy is often referred to as the "golden" trilepton channel as it is one of the most promising search channels for supersymmetry. In mSUGRA and similar models the lighter chargino, the two lightest neutralinos and the right-handed sleptons are, for most sets of parameters, considerably lighter than squarks and gluinos; additionally the trilepton final state has low Standard Model background at a hadron collider.

In this analysis electrons and muons are identified via their signals in the tracker, the calorimeter and the muon system. $\tau$ leptons can decay into an electron or a muon and be identified in the respective categories, but with branching ratio of approximately $50 \%$ $\tau$ leptons decay into a hadronic single-prong final state. To get acceptance to this decay we identify isolated tracks. 
We expect $0.88 \pm 0.05$ (stat) \pm 0.13 (syst) events from background processes and observe 1 event in the analysis channels with three leptons; for an expectation of $5.5 \pm 0.7$ (stat) \pm 0.9 (syst) background events 6 events are observed in the channels with two leptons and one isolated track.

As this analysis is not able to find evidence for the production of supersymmetric chargino-neutralino pairs, $95 \%$ confidence level limits on the production cross section and branching ratio $\sigma \times \mathrm{BR}$ are calculated. This analysis is the first chargino-neutralino search since LEP that is able to set a direct exclusion in mSUGRA. The exclusion is calculated as a function of the mSUGRA parameters $m_{0}$ and $m_{1 / 2}$.

A detailed study of the sensitivity of the analysis towards the full set of mSUGRA parameters is done and the important quantities for the ability of the analysis to set an exclusion are identified. It is shown that the ability to claim an exclusion mainly depends on the branching ratio $\operatorname{BR}\left(\tilde{\chi}_{2}^{0} \tilde{\chi}_{1}^{ \pm} \rightarrow 3\right.$ leptons $)$ and the average number of $\tau$ leptons in the final state. Based on these quantities a method for the generalization of the results of this analysis is developed. We split the analysis into channels for final states with $0,1,2$ and 3 $\tau$ leptons and determine the acceptance as a function of masses individually. A method to obtain exclusions for other possible realizations of supersymmetry is provided.

Parts of this thesis are published as arXiv:0808.2446v1 [hep-ex][67] (submitted to Phys. Rev. Lett) and arXiv:0808.1605v1 [hep-ph][68]. 


\section{References}

[1] W.-M. Yao et al., "Review of Particle Physics," Journal of Physics G 33 (2006) 1+.

[2] C. Burgess and G. Moore, The Standard Model: A Primer, pp. 79-81. Cambridge University Press, 2007.

[3] M. Veltman, Diagrammatica - The Path to Feynman Rules. Cambridge University Press, 1994.

[4] J. R. Ellis, G. Ridolfi, and F. Zwirner, "Higgs boson properties in the standard model and its supersymmetric extensions," Comptes Rendus Physique 8 (2007) 999-1012, arXiv hep-ph/0702114.

[5] D. H. Perkins, Introduction to High Energy Physics. Cambridge University Press, 2000 .

[6] S. Dimopoulos and D. Sutter, "The supersymmetric flavor problem," Nuclear Physics B 452 (October, 1995) 496-512.

[7] S. P. Martin, "A supersymmetry primer," arXiv hep-ph/9709356.

[8] K. Fujii, D. J. Miller, and A. Soni, eds., Linear Collider Physics in the new Millenium. World Scientific, 2005.

[9] A. Djouadi, "The Anatomy of Electro-Weak Symmetry Breaking. II: The Higgs bosons in the Minimal Supersymmetric Model," Phys. Rept. 459 (2008) 1-241, arXiv hep-ph/0503173.

[10] I. Aitchison, Supersymmetry in Particle Physics. Cambridge University Press, first ed., 2007.

[11] P. Binetruy, Supersymmetry, pp. 25-27. Oxford University Press, 2006.

[12] M. Drees, R. M. Godbole, and P. Roy, Theory and Phenomenology of Sparticles. World Scientific, 2004.

[13] LEP Electroweak Working Group and others, D. Abbaneo, et al., "A combination of preliminary electroweak measurements and constraints on the standard model," arXiv hep-ex/0212036.

[14] LEP Working Group for Higgs boson searches, R. Barate, et al., "Search for the standard model Higgs boson at LEP," Phys. Lett. B565 (2003) 61-75, arXiv hep-ex/0306033.

[15] M. Roos, Introduction to Cosmology. John Wiley \& Sons, Ltd, 2003. 
[16] WMAP Collaboration, G. Hinshaw et al., "Five-Year Wilkinson Microwave Anisotropy Probe (WMAP) Observations: Data Processing, Sky Maps, \& Basic Results," arXiv 0803.0732v1.

[17] E. Witten, "Supersymmetry and other Scenarios," in XXI International Symposium on Lepton and Photon Interactions at High Energies. 2003.

[18] The Accelerator Division of Fermilab, "Integrated Luminosity," 2008. http://www-bd.fnal.gov/pplot/today/DataSummaryTables.html as of Aug 20, 2008 .

[19] The Accelerator Division of Fermilab, "Run II parameter list," 2003. http://www-bd.fnal.gov/runII/parameters.pdf as of Aug 20, 2008.

[20] CDF Collaboration, P. T. Lukens, "The CDF IIb detector: Technical design report,". FERMILAB-TM-2198.

[21] CDF Collaboration, R. Blair et al., "The CDF-II detector: Technical design report,". FERMILAB-PUB-96-390-E.

[22] D. Glenzinski, "CDF Run II Detector Parameters," 2007. http://www-cdf.fnal.gov/internal/detectors/parameters.html as of Aug 20, 2008.

[23] S. Klimenko, J. Konigsberg, and T. Liss, "CDF Note 6314: Averaging of the inelastic cross-section measured by the CDF and the E811 experiments," 2003. $\mathrm{CDF} / \mathrm{DOC} / \mathrm{CDF} / \mathrm{CDFR} / 6314$.

[24] W. K. Sakumoto, "CDF Note 8318: Event $Z_{V T X} \leq 60 \mathrm{~cm}$ Cut Acceptance for Run II," 2006. CDF/ANAL/ELECTROWEAK/CDFR/8318.

[25] S. Rolli, "PerfIDia - an automatic set of tools to calculate Identification/Reconstruction/Trigger Efficiencies and Scale Factors," 2008. http://ncdf70.fnal.gov:8001/PerfIDia/PerfIDia.html as of Aug 20, 2008.

[26] V. Boisvert, "CDF Note 7939: Trigger Efficiencies for the High ET Central Electrons in Gen6," 2005. CDF/DOC/ELECTRON/CDFR/7939.

[27] U. Grundler, L. Lovas, and A. Taffard, "CDF Note 8618: High-Pt muons recommended cuts and efficiencies for Winter 2007," 2006.

$\mathrm{CDF} / \mathrm{ANAL} / \mathrm{TOP} / \mathrm{CDFR} / 8618$.

[28] U. Grundler, L. Lovas, and A. Taffard, "CDF Note 8262: High-Pt muons recommended cuts and efficiencies for Summer 2006," 2006.

$\mathrm{CDF} / \mathrm{ANAL} / \mathrm{TOP} / \mathrm{CDFR} / 8262$.

[29] S. Rolli and L. Lovas, "CDF Note 9085: Muon ID, Reconstruction and Trigger Efficiencies and Scale Factors for Period 9-12 data," 2007. CDF/ANAL/MUON/CDFR/9085.

[30] M. Gold, V. Rekovic, and J. Strologas, "CDF Note 8308: Level 1, 2, and 3 Low pT Muon Trigger Efficiencies in gen6 Data for the SUSY Trilepton Searches," 2006. CDF/ANAL/EXOTIC/CDFR/8308. 
[31] T. Sjöstrand, P. Edén, C. Friberg, L. Lönnblad, G. Miu, S. Mrenna, and E. Norrbin, "High-energy-physics event generation with PYTHIA 6.1," Computer Physics Communications 135 (2001), no. 2, 238-259.

[32] CTEQ Collaboration, H. L. Lai et al., "Global QCD analysis of parton structure of the nucleon: CTEQ5 parton distributions," Eur. Phys. J. C12 (2000) 375-392, arXiv hep-ph/9903282.

[33] H. Baer, F. E. Paige, S. D. Protopescu, and X. Tata, "ISAJET 7.69: A Monte Carlo Event Generator for $p p, \bar{p} p$, and $e^{+} e^{-}$Reactions," arXiv hep-ph/0312045.

[34] P. Golonka, B. Kersevan, T. Pierzchala, E. Richter-Wạs, Z. Wạs, and M. Worek, "The tauola-photos-F environment for the TAUOLA and PHOTOS packages, release II," Computer Physics Communications 174 (2006), no. 10, 818-835.

[35] W. Beenakker, M. Klasen, M. Krämer, T. Plehn, M. Spira, and P. M. Zerwas, "Production of Charginos, Neutralinos, and Sleptons at Hadron Colliders," Phys. Rev. Lett. 83 (Nov, 1999) 3780-3783.

[36] V. D. Barger and C. Kao, "Trilepton signature of minimal supergravity at the upgraded Tevatron," Phys. Rev. D60 (1999) 115015, arXiv hep-ph/9811489.

[37] T. Plehn and M. Spira private communication.

[38] S. Mrenna, G. L. Kane, G. D. Kribs, and J. D. Wells, "Possible signals of constrained minimal supersymmetry at a high luminosity Fermilab Tevatron collider," Phys. Rev. D53 (1996) 1168-1180, arXiv hep-ph/9505245.

[39] C. Hays, P. Tamburello, A. Kotwal, P. Wittich, and R. Snider, "CDF Note 6992: The COT Pattern Recognition Algorithm and Offline Code," 2004.

CDF/DOC/TRACKING/CDFR/6992.

[40] P. Azzi, G. Busetto, P. Gatti, and A. Ribon, "CDF Note 5562: Histogram Tracking in the COT," 2001. CDF/DOC/TRACKING/CDFR/5562.

[41] T. Spreitzer, C. Mills, and J. Incandela, "CDF Note 7950: Electron Identification in Offline Release 6.1.2," 2005. CDF/DOC/ELECTRON/CDFR/7950.

[42] R. G. Wagner, "CDF Note 6249: Electron Identification for Run II: Understanding and Using Lshr," 2003. CDF/DOC/ELECTRON/CDFR/6249.

[43] A. Bhatti et al., "Determination of the jet energy scale at the Collider Detector at Fermilab," Nucl. Instrum. Meth. A566 (2006) 375-412, arXiv hep-ex/0510047.

[44] S. Dube, Search for Supersymmetry at the Tevatron using the Trilepton signature. PhD in Physics, Graduate School New Brunswick, Rutgers University, 2008.

[45] A. Taffard, "CDF Note 6100: Run II Cosmic Ray tagger," 2003. $\mathrm{CDF} / \mathrm{PUB} / \mathrm{MUON} / \mathrm{PUBLIC} / 6100$.

[46] A. Attal and A. Canepa, "CDF Note 8073: Photon Conversion Removal Efficiency," 2006. CDF/ANAL/ELECTRON/CDFR/8073. 
[47] S. Dube, J. Zhou, A. Lath, and S. Somalwar, "CDF Note 8445: Searches for Chargino-Neutralino Production in mSUGRA model in a Di-electron + Track Channel," 2006. CDF/DOC/EXOTIC/CDFR/8445.

[48] G. Manca, M. Griffiths, and B. Heinemann, "CDF Note 8389: Search for Associated Production of Chargino and Neutralino in the Tri-Lepton Final State using the High $p_{T}$ Electron Trigger - Update with $1 \mathrm{fb}^{-1}$," 2006.

CDF/ANAL/EXOTIC/CDFR/8389.

[49] J. Zhou, S. Dube, A. Lath, and S. Somalwar, "CDF Note 7478: Search for Chargino-Neutralino Pair Production in Dielectron+Track Channel," 2005. CDF/ANAL/EXOTIC/CDFR/7478.

[50] CDF Collaboration, A. Abulencia et al., "Measurements of inclusive W and Z cross sections in $p \bar{p}$ collisions at $\sqrt{s}=1.96 \mathrm{TeV}$," Journal of Physics G: Nuclear and Particle Physics 34 (2007), no. 12, 2457-2544.

[51] M. Griffiths, B. Heinemann, and G. Manca, "CDF Note 7470: Fake Rate For Low- $p_{T}$ Leptons," 2005. CDF/ANAL/EXOTIC/CDFR/7470.

[52] S. Dube, J. Glatzer, A. Sood, and S. Somalwar, "CDF Note 9097: Unified Search for Chargino-Neutralino Production with $2 \mathrm{fb}^{-1}, " 2007$. CDF/ANAL/EXOTIC/CDFR/9097.

[53] S.-C. Hsu, E. Lipeles, M. Neubauer, M. Norman, R. Vanguri, and F. Würthwein, "CDF Note 8924: Search for ZZ in the 4 lepton and dilepton + MET channel and update of WZ in the trilepton +MET," 2007.

CDF/ANAL/ELECTROWEAK/CDFR/8924.

[54] B. Jayatilaka, A. Kotwal, R. Shekhar, D. Whiteson, and M. Tecchio, "CDF Note 9098: Measurement of the top quark mass in the dilepton channel 2.0/fb with the Matrix-Element Method," 2007. CDF/PHYS/TOP/CDFR/9098.

[55] ATLAS Detector and Physics Performance, Technical Design Report, pp. 816-863. ATLAS Collaboration, 1999.

[56] CMS Physics Technical Design Report: Physics Performance, pp. 407-410. CMS Collaboration, 2006.

[57] CDF Statistics Committee, "Statistics Software Website." http://www-cdf.fnal.gov/physics/statistics/statistics_software.html as of Aug 20, 2008.

[58] T. Junk, "CDF Note 8128: Sensitivity, Exclusion and Discovery with Small Signals, Large Backgrounds, and Large Systematic Uncertainties," 2007. CDF/DOC/STATISTICS/PUBLIC/8128.

[59] LEP2 SUSY Working Group, "Combined LEP Chargino Results," July, 2008. http://lepsusy.web.cern.ch/lepsusy/ as of Aug 20, 2008.

[60] CDF Collaboration, A. Abulencia et al., "Inclusive search for new physics with like-sign dilepton events in $p \bar{p}$ collisions at $\sqrt{s}=1.96-\mathrm{TeV}, "$ Phys. Rev. Lett. 98 (2007) 221803, arXiv hep-ex/0702051. 
[61] CDF Collaboration, T. Aaltonen et al., "Search for chargino-neutralino production in p-pbar collisions at $1.96 \mathrm{TeV}$ with high pT leptons," Phys. Rev. D77 (2008) 052002, arXiv 0711.3161.

[62] J. Strologas and M. Gold, "CDF Note 9311: Search for new physics in the $\mathrm{mu}+\mathrm{mu}+\mathrm{e} / \mathrm{mu}$ channel with low-pT lepton threshold at CDF," 2008. $\mathrm{CDF} / \mathrm{PUB} / \mathrm{CDF} / \mathrm{CDFR} / 9311$.

[63] S. Dube, J. Zhou, and S. Somalwar, "CDF Note 8529: Search for Chargino-Neutralino Production in e+e+track final state," 2006. CDF/PUB/EXOTIC/PUBLIC/8529.

[64] CDF Collaboration, T. Aaltonen et al., "Search for chargino-neutralino production in $p \bar{p}$ collisions at $\sqrt{s}=1.96 \mathrm{TeV}$," Phys. Rev. Lett. 99 (2007) 191806, arXiv 0707.2362.

[65] DØ Collaboration, V. M. Abazov et al., "Search for supersymmetry via associated production of charginos and neutralinos in final states with three leptons," Phys. Rev. Lett. 95 (2005) 151805, arXiv hep-ex/0504032.

[66] V. M. Abazov et al., "Search for the Associated Production of Chargino and Neutralino in Final States with three Leptons with the DØ detector," 2007. DØ Note 5348-Conf.

[67] CDF Collaboration, T. Aaltonen et al., "Search for Supersymmetry in $p \bar{p}$ Collisions at $\sqrt{s}=1.96 \mathrm{TeV}$ Using the Trilepton Signature of Chargino-Neutralino Production," arXiv 0808.2446. Submitted to Phys. Rev. Lett.

[68] S. Dube, J. Glatzer, S. Somalwar, and A. Sood, "An Interpretation of Tevatron SUSY Trilepton Search Results in mSUGRA and in a Model-independent Fashion," arXiv 0808.1605. 


\section{Appendix A Control Regions}

The number of observed and expected events for dilepton and trilepton control regions is documented in Tables A.1 and A.2. The control regions are defined in Table 4.7.

Table A.1: Number of expected and observed events in the analysis channels for the trilepton control regions

\begin{tabular}{|c|c|c|c|}
\hline Control Region & Channel & Predicted Background & Observed \\
\hline \multicolumn{4}{|c|}{ Trilepton Control Regions } \\
\hline \multirow{5}{*}{ !Zlo } & $l_{t} l_{t} l_{t}$ & $6.3 \pm 2.7$ & 9 \\
\hline & $l_{t} l_{t} l_{l}$ & $2.2 \pm 1.5$ & 3 \\
\hline & $l_{t} l_{l} l_{l}$ & $1.4 \pm 1.3$ & 0 \\
\hline & $l_{t} l_{t} T$ & $88 \pm 13$ & 72 \\
\hline & $l_{t} l_{l} T$ & $34 \pm 7$ & 31 \\
\hline \multirow{5}{*}{ Zlo } & $l_{t} l_{t} l_{t}$ & $10.8 \pm 4.2$ & 8 \\
\hline & $l_{t} l_{t} l_{l}$ & $4.9 \pm 2.5$ & 6 \\
\hline & $l_{t} l_{l} l_{l}$ & $2.8 \pm 1.9$ & 3 \\
\hline & $l_{t} l_{t} T$ & $223 \pm 26$ & 218 \\
\hline & $l_{t} l_{l} T$ & $195 \pm 26$ & 183 \\
\hline \multirow{5}{*}{ Zhi } & $l_{t} l_{t} l_{t}$ & $2.7 \pm 1.7$ & 0 \\
\hline & $l_{t} l_{t} l_{l}$ & $1.7 \pm 1.3$ & 2 \\
\hline & $l_{t} l_{l} l_{l}$ & $1.6 \pm 1.3$ & 2 \\
\hline & $l_{t} l_{t} T$ & $26.8 \pm 6.0$ & 34 \\
\hline & $l_{t} l_{l} T$ & $27.7 \pm 6.3$ & 23 \\
\hline
\end{tabular}


Table A.2: Number of expected and observed events in the analysis channels for the dilepton control regions

\begin{tabular}{|c|c|c|c|}
\hline Control Region & Channel & Predicted Background & Observed \\
\hline \hline \multicolumn{2}{|c|}{ Dilepton Control Regions } \\
\hline & $l_{t} l_{t}$ & $51150 \pm 2034$ & 51042 \\
& $e_{t} e_{t}$ & $31222 \pm 1710$ & 31074 \\
& $\mu_{t} \mu_{t}$ & $19895 \pm 1102$ & 19942 \\
& $l_{t} l_{l}$ & $42288 \pm 1868$ & 41883 \\
& $e_{t} e_{l}$ & $10591 \pm 664$ & 10235 \\
& $\mu_{t} \mu_{l}$ & $30947 \pm 1728$ & 30958 \\
\hline & $l_{t} l_{t}$ & $16352 \pm 716$ & 15966 \\
& $e_{t} e_{t}$ & $10399 \pm 617$ & 10033 \\
& $\mu_{t} \mu_{t}$ & $5290 \pm 352$ & 5198 \\
& $l_{t} l_{l}$ & $7198 \pm 300$ & 7069 \\
& $e_{t} e_{l}$ & $1855 \pm 114$ & 1890 \\
& $\mu_{t} \mu_{l}$ & $4550 \pm 261$ & 4482 \\
\hline
\end{tabular}




\section{Appendix B \\ Limitations and Versions of PYTHIA and ISAJET}

The combination of a ISAJET and PYTHIA, where a SUSY Les Houches Accord File is passed, is not considering mass widths for the calculation of branching ratios. This leads to problems in the region, where the transition from off-shell three-body decays (region $\mathrm{A}$ in Fig. B.2) to on-shell sequential two-body decay (region B in Fig. B.2) happens. In Fig. B.1 the branching ratio into three leptons is shown in this region. Due to the fact that no mass width is considered the decay changes from two-body to three-body once the three-body decay is kinematically allowed. This leads to the artificial behavior of the branching ratio for $94 \mathrm{GeV} / \mathrm{c}^{2}<m_{0}<97 \mathrm{GeV} / \mathrm{c}^{2}$. Once the decay via on-shell particles is possible the branching ratio of the according off-shell decay is set to 0 and the branching ratio for the on-shell decay is calculated. As the mass of the slepton is approximately the mass of the chargino and neutralino, the available phase space for the decay products is small and the branching ratio drops. If mass widths would have been considered there would not be one point where the decay transition happens and a smooth curve for the branching ratio would be the case.

In Fig. B.2 a line shows the region where $m\left(\tilde{\chi}_{1}^{ \pm}\right)=m\left(\tilde{\chi}_{1}^{0}\right)+m\left(W^{ \pm}\right)$. It can be see that around this line the branching ratio into three leptons is increasing. The reason can be seen in Fig. B.3. At $m_{0}=253 \mathrm{GeV} / \mathrm{c}^{2}$ the decay $\tilde{\chi}_{1}^{ \pm} \rightarrow \tilde{\chi}_{1}^{0} W^{ \pm}$becomes kinematically allowed and the decay via off-shell $W^{ \pm *}$ is turned off. As $m\left(\tilde{\chi}_{1}^{ \pm}\right) \approx m\left(\tilde{\chi}_{1}^{0}\right)+m\left(W^{ \pm}\right)$the phase space for this decay is small and the branching ratio of the decay via an on-shell stau slepton increases until the phase space of the decay via on-shell $W^{ \pm}$is big enough.

For this analysis different versions of PYTHIA and ISAJET have been used. The Monte Carlo Ntuples have been generated with PYTHIA 6.216 and ISAJET 7.51 while the calculation of the cross section and the branching ratio into three leptons was done with PYTHIA 6.409 


\section{Branching ratios and masses}

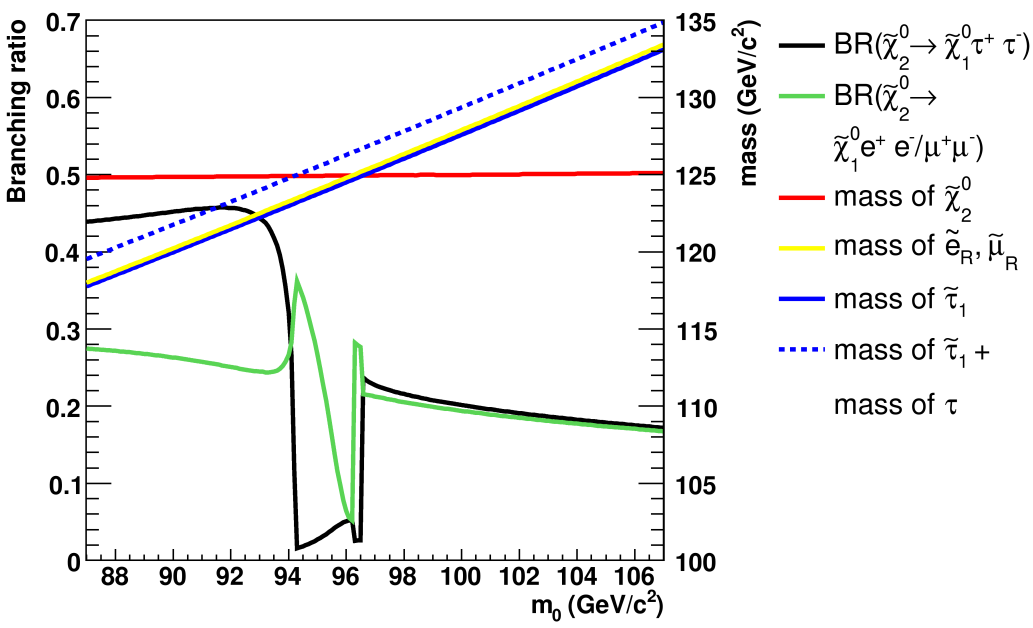

Figure B.1: Branching ratio of chargino and neutralino into three leptons at the transition from off-shell three-body decay to on-shell sequential two-body decay. We don't differentiate between a decay via on-shell slepton, via off-shell slepton and via off-shell $W^{ \pm *}$ or $Z^{0 *}$ boson for the branching ratio in this figure.

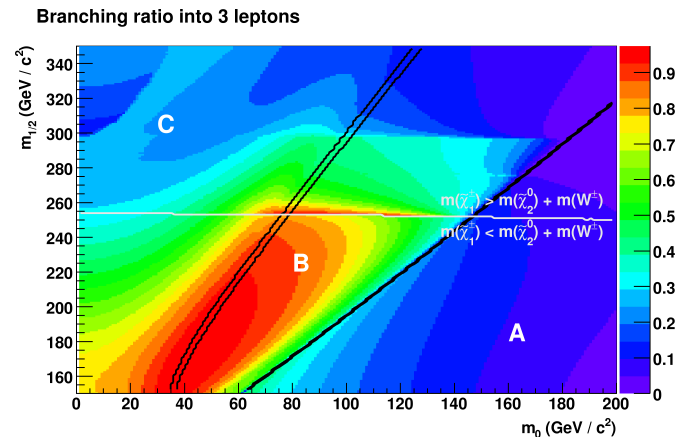

Figure B.2: Branching ratio of chargino and neutralino into three leptons

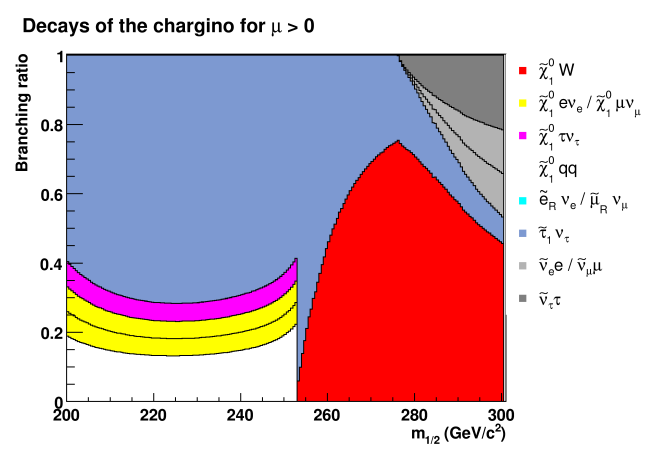

Figure B.3: Branching ratios for the chargino as a function of $m_{1 / 2}$ at benchmark point $\mathrm{BP} 1$ with $\mu>0$ 
and ISAJET 7.75. In the CDF Monte Carlo production framework PYTHIA 6.216 was tuned to match the collected data, but no tune for more recent versions of PYTHIA is available. As newer ISAJET versions are not usable with PYTHIA 6.216, ISAJET 7.51 had to be used for the Monte Carlo production. It was decided to use the recent versions PYTHIA 6.409 and ISAJET 7.75, where no tune is needed. 


\section{Appendix C}

\section{Average Number of $\tau$ Leptons per Event}

The trilepton analysis has channels with 3 genuine leptons and channels with 2 genuine leptons and one track. As the channels with a track have a worse signal to background ratio the sensitivity of the analysis is dependent on how many events fall into the two types of channels. The average number of $\tau$ leptons per event is a measure for this.

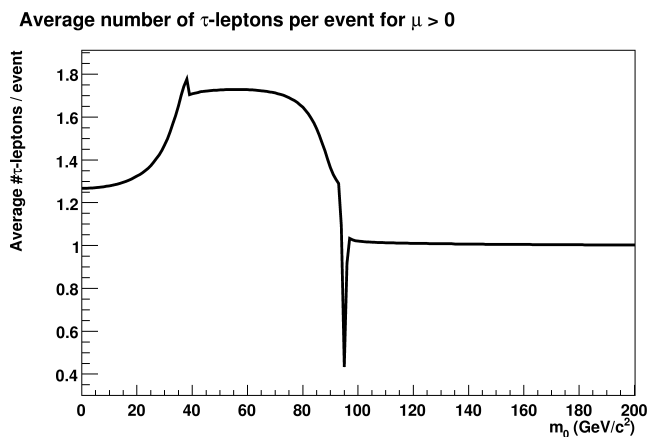

Figure C.1: Average number of $\tau$ leptons per event as a function of $m_{0}$ at benchmark point BP1 with $\mu>0$

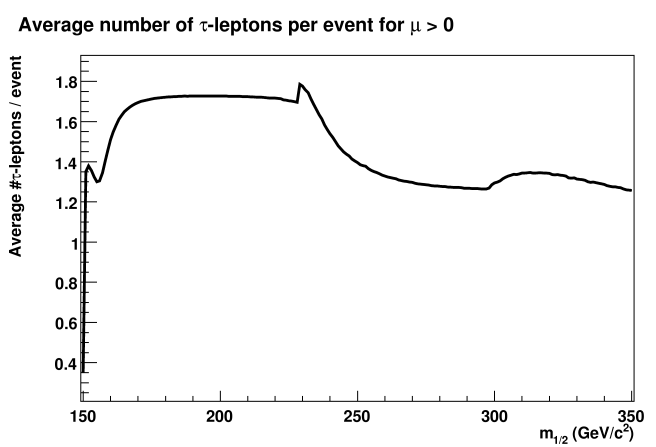

Figure C.3: Average number of $\tau$ leptons per event as a function of $m_{1 / 2}$ at benchmark point BP1 with $\mu>0$

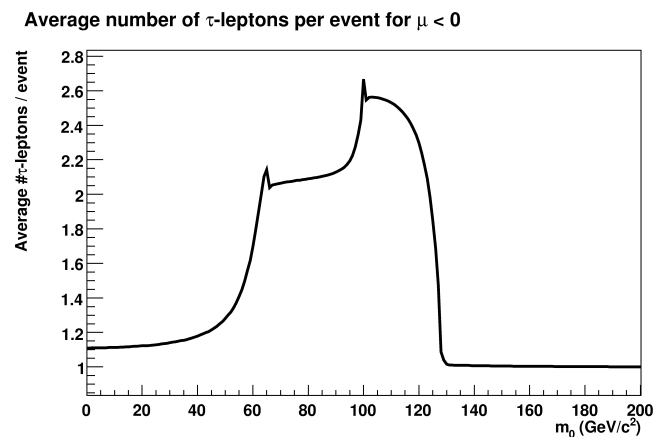

Figure C.2: Average number of $\tau$ leptons per event as a function of $m_{0}$ at benchmark point $\mathrm{BP} 3$ with $\mu<0$

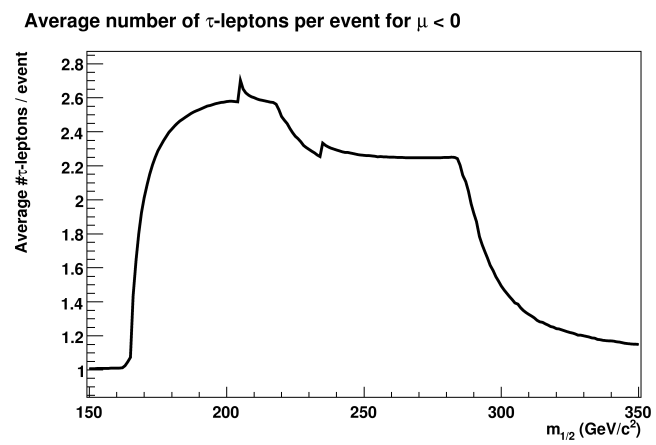

Figure C.4: Average number of $\tau$ leptons per event as a function of $m_{1 / 2}$ at benchmark point BP3 with $\mu<0$ 


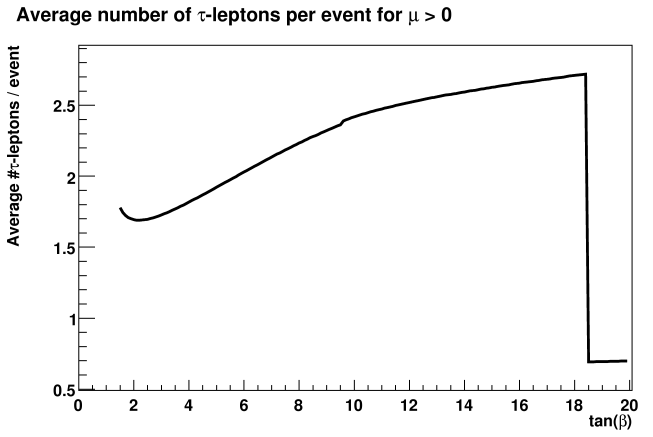

Figure C.5: Average number of $\tau$ leptons per event as a function of $\tan \beta$ at benchmark point $\mathrm{BP} 1$ with $\mu>0$

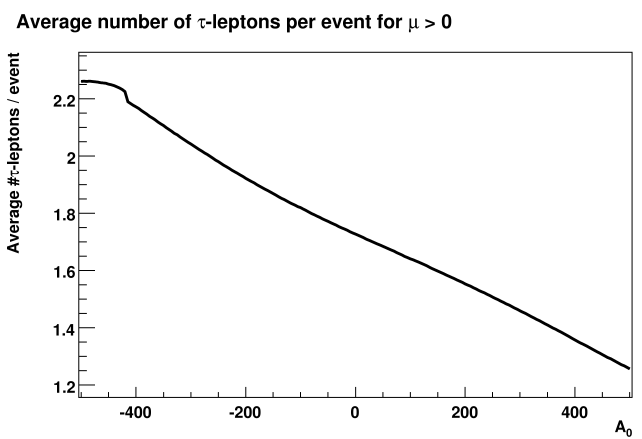

Figure C.7: Average number of $\tau$ leptons per event as a function of $A_{0}$ at benchmark point $\mathrm{BP} 1$ with $\mu>0$

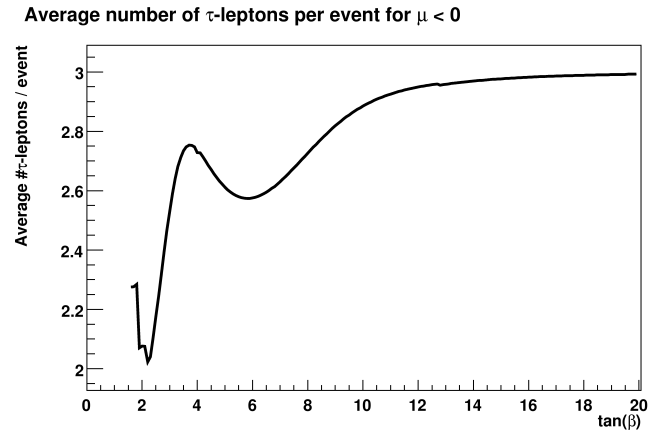

Figure C.6: Average number of $\tau$ leptons per event as a function of $\tan \beta$ at benchmark point BP3 with $\mu<0$

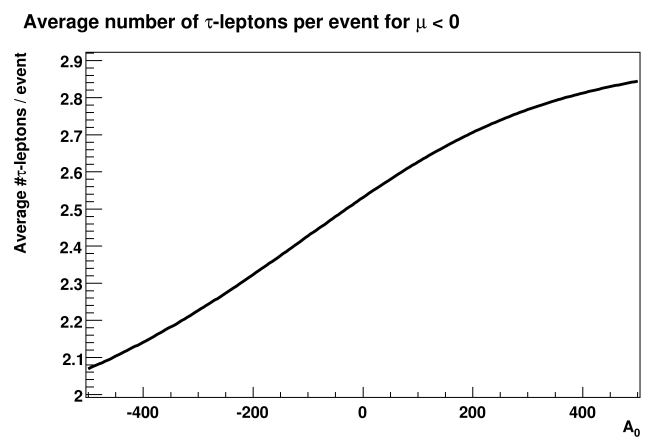

Figure C.8: Average number of $\tau$ leptons per event as a function of $A_{0}$ at benchmark point BP3 with $\mu<0$ 


\section{Appendix D \\ Monte Carlo Sample Generation for the Model-Independent Interpretation}

In this section the generation of a Monte Carlo sample with 3 lepton final states including $i=0,1,2,3 \tau$ leptons as needed in section 6 is described. As the chargino can decay into no or one $\tau$ lepton and the neutralino into no or two $\tau$ leptons it can be concluded, that for final states with

- $0 \tau$ leptons the chargino decayed into an electron or a muon and the neutralino decayed into electrons or muons,

- $1 \tau$ lepton the chargino decayed into a $\tau$ lepton and the neutralino decayed into electrons or muons,

- $2 \tau$ leptons the chargino decayed into an electron or a muon and the neutralino decayed into $\tau$ leptons,

- $3 \tau$ leptons the chargino decayed into a $\tau$ lepton and the neutralino decayed into $\tau$ leptons.

In section 6 the description was limited to the cases where

1. $m\left(\tilde{\chi}_{1}^{0}\right)<m\left(\tilde{\chi}_{1}^{ \pm}\right) \approx m\left(\tilde{\chi}_{2}^{0}\right)<$ mass of all other supersymmetric particles

2. $m\left(\tilde{\chi}_{1}^{0}\right)<m\left(\tilde{l}_{R}^{ \pm}\right)<m\left(\tilde{\chi}_{1}^{ \pm}\right) \approx m\left(\tilde{\chi}_{2}^{0}\right)<$ mass of all other supersymmetric particles, where $\tilde{l}_{R}^{ \pm}$stands for the lighter selectron, smuon or stau.

As the mass parameters

1. chargino mass $m\left(\tilde{\chi}_{1}^{ \pm}\right)$and thus implicitly the mass of the neutralino, 
2. $\Delta M_{1}=m\left(\tilde{\chi}_{1}^{ \pm}\right)-m\left(\tilde{l}_{R}^{ \pm}\right)$,

3. $\Delta M_{2}=m\left(\tilde{\chi}_{1}^{ \pm}\right)-m\left(\tilde{\chi}_{1}^{0}\right)$.

are given the two case are equivalent to

1. $\Delta M_{1}<0$,

2. $\Delta M_{1}>0$.

It can be assumed that in case 1 the decay of the chargino and neutralino are as follows

$$
\begin{aligned}
& \tilde{\chi}_{1}^{ \pm} \rightarrow \tilde{\chi}_{1}^{0} W^{ \pm *} \rightarrow \tilde{\chi}_{1}^{0} l^{ \pm} \nu_{l} \text { or } \\
& \tilde{\chi}_{1}^{ \pm} \rightarrow \tilde{l}_{R}^{ \pm *} \nu_{l} \rightarrow \tilde{\chi}_{1}^{0} l^{ \pm} \nu_{l} \\
& \tilde{\chi}_{2}^{0} \rightarrow \tilde{\chi}_{1}^{0} Z^{0 *} \rightarrow \tilde{\chi}_{1}^{0} l^{+} l^{-} \quad \text { or } \\
& \tilde{\chi}_{2}^{0} \rightarrow \tilde{l}_{R}^{ \pm *} l^{\mp} \rightarrow \tilde{\chi}_{1}^{0} l^{+} l^{-} .
\end{aligned}
$$

It is not important if the decay goes via an off-shell slepton or an off-shell $W^{ \pm *}$ or $Z^{0 *}$ boson; in both cases a three-body decay is taking place. In case 2 the decay is mostly

$$
\begin{aligned}
& \tilde{\chi}_{1}^{ \pm} \rightarrow \tilde{l}_{R}^{ \pm} \nu_{l} \rightarrow \tilde{\chi}_{1}^{0} l^{ \pm} \nu_{l} \\
& \tilde{\chi}_{2}^{0} \rightarrow \tilde{l}_{R}^{ \pm} l^{\mp} \rightarrow \tilde{\chi}_{1}^{0} l^{+} l^{-},
\end{aligned}
$$

where the sleptons are on shell. For most models it is sufficient to assume $100 \%$ branching ratio via on-shell slepton.

For every set of mass parameters four Monte Carlo samples with 0,1,2,3 $\tau$ leptons in the final state are produced. We set the masses of $\tilde{\chi}_{1}^{0}, \tilde{\chi}_{2}^{0}, \tilde{\chi}_{1}^{ \pm}, \tilde{e}_{R}^{ \pm}, \tilde{\mu}_{R}^{ \pm}$and $\tilde{\tau}_{1}^{ \pm}$to

$$
\begin{aligned}
& m\left(\tilde{\chi}_{1}^{0}\right)=m\left(\tilde{\chi}_{1}^{ \pm}\right)-\Delta M_{2}, \\
& m\left(\tilde{\chi}_{2}^{0}\right)=m\left(\tilde{\chi}_{1}^{ \pm}\right), \\
& m\left(\tilde{e}_{R}^{ \pm}\right)=m\left(\tilde{\mu}_{R}^{ \pm}\right)=m\left(\tilde{\tau}_{1}^{ \pm}\right)=m\left(\tilde{\chi}_{1}^{ \pm}\right)-\Delta M_{1} .
\end{aligned}
$$

The masses of all other supersymmetric particles are set to high values. For the decay of the sleptons we set all branching ratios to 0 except $\tilde{l}^{ \pm} \rightarrow \tilde{\chi}_{1}^{0} l^{ \pm}$to which the branching ratio $100 \%$ is assigned. For the chargino and the neutralino we set the branching ratios of all decay channels except the ones mentioned later to 0 . 


\section{Branching Ratios for the Case $\Delta M_{1}>0$}

For the sample with $i=0$ we set the branching ratios

$$
\begin{aligned}
& \operatorname{BR}\left(\tilde{\chi}_{1}^{ \pm} \rightarrow \tilde{\chi}_{1}^{0} e^{ \pm} \nu_{e}\right)=50 \% \\
& \operatorname{BR}\left(\tilde{\chi}_{1}^{ \pm} \rightarrow \tilde{\chi}_{1}^{0} \mu^{ \pm} \nu_{\mu}\right)=50 \% \\
& \operatorname{BR}\left(\tilde{\chi}_{2}^{0} \rightarrow \tilde{\chi}_{1}^{0} e^{+} e^{-}\right)=50 \% \\
& \operatorname{BR}\left(\tilde{\chi}_{2}^{0} \rightarrow \tilde{\chi}_{1}^{0} \mu^{+} \mu^{-}\right)=50 \% .
\end{aligned}
$$

For the sample with $i=1$ we set the branching ratios

$$
\begin{gathered}
\operatorname{BR}\left(\tilde{\chi}_{1}^{ \pm} \rightarrow \tilde{\chi}_{1}^{0} \tau^{ \pm} \nu_{\tau}\right)=100 \% \\
\operatorname{BR}\left(\tilde{\chi}_{2}^{0} \rightarrow \tilde{\chi}_{1}^{0} e^{+} e^{-}\right)=50 \% \\
\operatorname{BR}\left(\tilde{\chi}_{2}^{0} \rightarrow \tilde{\chi}_{1}^{0} \mu^{+} \mu^{-}\right)=50 \% .
\end{gathered}
$$

For the sample with $i=2$ we set the branching ratios

$$
\begin{aligned}
\operatorname{BR}\left(\tilde{\chi}_{1}^{ \pm} \rightarrow \tilde{\chi}_{1}^{0} e^{ \pm} \nu_{e}\right) & =50 \% \\
\operatorname{BR}\left(\tilde{\chi}_{1}^{ \pm} \rightarrow \tilde{\chi}_{1}^{0} \mu^{ \pm} \nu_{\mu}\right) & =50 \% \\
\operatorname{BR}\left(\tilde{\chi}_{2}^{0} \rightarrow \tilde{\chi}_{1}^{0} \tau^{+} \tau^{-}\right) & =100 \% .
\end{aligned}
$$

For the sample with $i=3$ we set the branching ratios

$$
\begin{aligned}
& \operatorname{BR}\left(\tilde{\chi}_{1}^{ \pm} \rightarrow \tilde{\chi}_{1}^{0} \tau^{ \pm} \nu_{\tau}\right)=100 \% \\
& \operatorname{BR}\left(\tilde{\chi}_{2}^{0} \rightarrow \tilde{\chi}_{1}^{0} \tau^{+} \tau^{-}\right)=100 \%
\end{aligned}
$$

\section{Branching Ratios for the Case $\Delta M_{1}<0$}

For the sample with $i=0$ we set the branching ratios

$$
\begin{aligned}
\operatorname{BR}\left(\tilde{\chi}_{1}^{ \pm} \rightarrow \tilde{e}_{R}^{ \pm} \nu_{e}\right) & =50 \% \\
\operatorname{BR}\left(\tilde{\chi}_{1}^{ \pm} \rightarrow \tilde{\mu}_{R}^{ \pm} \nu_{\mu}\right) & =50 \% \\
\operatorname{BR}\left(\tilde{\chi}_{2}^{0} \rightarrow \tilde{e}_{R}^{ \pm} e^{\mp}\right) & =50 \% \\
\operatorname{BR}\left(\tilde{\chi}_{2}^{0} \rightarrow \tilde{\mu}_{R}^{ \pm} \mu^{\mp}\right) & =50 \% .
\end{aligned}
$$


For the sample with $i=1$ we set the branching ratios

$$
\begin{aligned}
\operatorname{BR}\left(\tilde{\chi}_{1}^{ \pm} \rightarrow \tilde{\tau}_{1}^{ \pm} \nu_{\tau}\right) & =100 \% \\
\operatorname{BR}\left(\tilde{\chi}_{2}^{0} \rightarrow \tilde{e}_{R}^{ \pm} e^{\mp}\right) & =50 \% \\
\operatorname{BR}\left(\tilde{\chi}_{2}^{0} \rightarrow \tilde{\mu}_{R}^{ \pm} \mu^{\mp}\right) & =50 \% .
\end{aligned}
$$

For the sample with $i=2$ we set the branching ratios

$$
\begin{gathered}
\operatorname{BR}\left(\tilde{\chi}_{1}^{ \pm} \rightarrow \tilde{e}_{R}^{ \pm} \nu_{e}\right)=50 \% \\
\operatorname{BR}\left(\tilde{\chi}_{1}^{ \pm} \rightarrow \tilde{\mu}_{R}^{ \pm} \nu_{\mu}\right)=50 \% \\
\operatorname{BR}\left(\tilde{\chi}_{2}^{0} \rightarrow \tilde{\tau}_{1}^{ \pm} \tau^{\mp}\right)=100 \% .
\end{gathered}
$$

For the sample with $i=3$ we set the branching ratios

$$
\begin{aligned}
& \operatorname{BR}\left(\tilde{\chi}_{1}^{ \pm} \rightarrow \tilde{\tau}_{1}^{ \pm} \nu_{\tau}\right)=100 \% \\
& \operatorname{BR}\left(\tilde{\chi}_{2}^{0} \rightarrow \tilde{\tau}_{1}^{ \pm} \tau^{\mp}\right)=100 \%
\end{aligned}
$$

For the case that $\Delta M_{1}<0$ and a significant branching ratio of the decays $\tilde{\chi}_{2}^{0} \rightarrow \tilde{\chi}_{1}^{0} l^{+} l^{-}$ or $\tilde{\chi}_{1}^{ \pm} \rightarrow \tilde{\chi}_{1}^{0} l^{+} l^{-}$, it is possible to use 8 samples, where four samples are produced for $\tilde{\chi}_{1}^{ \pm}$, $\Delta M_{1}, \Delta M_{2}$ and four samples where $\Delta M_{1}$ is set to a positive value. The total acceptance for case $\mathrm{i}$ is then the acceptance obtained from both samples weighted by the appropriate branching ratio

$$
A_{\text {total }}=\mathrm{BR}(\text { off-shell decay }) \times A_{\text {off-shell }}+\mathrm{BR}(\text { on-shell decay }) \times A_{\text {on-shell }} \text {. }
$$




\section{Vita}

Julian Glatzer was born in Eberbach, Germany on October 18, 1984. He graduated from Nicolaus-Kistner-Gymnasium Mosbach with the German high school diploma "Abitur" in 2004. He studied at the University of Würzburg with major physics from 2004 to 2007 and with major mathematics from 2005 to 2007. He completed his undergraduate studies in physics with "Vordiplom" in 2006 and in mathematics with "Vordiplom" in 2007. In 2007

he joined the graduate program of Rutgers University and started work with Prof. Sunil Somalwar in the CDF Collaboration.

Permanent address: Julian Glatzer, Tarunstr. 176, 74821 Mosbach, Germany 